\title{
Imaging strategies towards an optimization of ovarian cancer therapy
}

Citation for published version (APA):

Rutten, I. J. G. (2017). Imaging strategies towards an optimization of ovarian cancer therapy. [Doctoral Thesis, Maastricht University]. Datawyse / Universitaire Pers Maastricht. https://doi.org/10.26481/dis.20170713ir

Document status and date:

Published: 01/01/2017

DOI:

10.26481/dis.20170713ir

Document Version:

Publisher's PDF, also known as Version of record

\section{Please check the document version of this publication:}

- A submitted manuscript is the version of the article upon submission and before peer-review. There can be important differences between the submitted version and the official published version of record.

People interested in the research are advised to contact the author for the final version of the publication, or visit the DOI to the publisher's website.

- The final author version and the galley proof are versions of the publication after peer review.

- The final published version features the final layout of the paper including the volume, issue and page numbers.

Link to publication

\footnotetext{
General rights rights.

- You may freely distribute the URL identifying the publication in the public portal. please follow below link for the End User Agreement:

www.umlib.nl/taverne-license

Take down policy

If you believe that this document breaches copyright please contact us at:

repository@maastrichtuniversity.nl

providing details and we will investigate your claim.
}

Copyright and moral rights for the publications made accessible in the public portal are retained by the authors and/or other copyright owners and it is a condition of accessing publications that users recognise and abide by the legal requirements associated with these

- Users may download and print one copy of any publication from the public portal for the purpose of private study or research.

- You may not further distribute the material or use it for any profit-making activity or commercial gain

If the publication is distributed under the terms of Article $25 \mathrm{fa}$ of the Dutch Copyright Act, indicated by the "Taverne" license above, 
Imaging strategies towards an optimization of ovarian cancer therapy 
(C) Iris Rutten, Maastricht 2017

Een aantal van de studies in dit proefschrift ontvingen financiële ondersteuning van het Academisch Fonds, Maastricht.

Kaftontwerp: Ralph Blomberg

Printing: Datawyse | Universitaire Pers Maastricht

ISBN 9789461597090

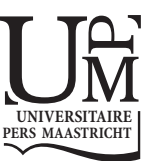




\title{
IMAGING STRATEGIES TOWARDS AN OPTIMIZATION OF OVARIAN CANCER THERAPY
}

\author{
PROEFSCHRIFT \\ ter verkrijging van de graad van doctor aan de Universiteit Maastricht, \\ op gezag van de Rector Magnificus, Prof. dr. Rianne M. Letschert, \\ volgens het besluit van het College van Decanen, \\ in het openbaar te verdedigen \\ op donderdag 13 juli 2017 om 10:00 uur \\ door
}

Iris Josephina Genoveva Rutten 


\section{Promotores}

Prof. dr. R.F.P.M. Kruitwagen

Prof. dr R.G.H. Beets-Tan

\section{Copromotor}

Dr. T. Van Gorp

\section{Beoordelingscommissie}

Prof. dr. F. Ramaekers (voorzitter)

Prof. Dr. F. Amant (Academisch ziekenhuis Leuven, België)

Prof. dr. C.H.C. Dejong

Prof. dr. A.M.W.J. Schols

Prof. Dr. V. Vandecaveye (Academisch ziekenhuis Leuven, België) 


\section{Contents}

Chapter 1 General introduction

\section{Part I CT-BASED ASSESSMENT OF SKELETAL MUSCLE LOSS AND OVARIAN CANCER SURVIVAL}

Chapter 2 Loss of skeletal muscle during neoadjuvant chemotherapy is related to decreased survival in ovarian cancer patients. Journal of Cachexia, Sarcopenia and Muscle (2016), 7: 458-466

Chapter 3 The influence of sarcopenia on survival and surgical complications in ovarian cancer patients undergoing primary debulking surgery. European Journal of Surgical Oncology (2016) 43(4):717-724

Chapter 4 Psoas muscle area is not representative of total skeletal muscle area in the assessment of sarcopenia in ovarian cancer. Accepted, Journal of Cachexia, Sarcopenia and Muscle

Part II CURRENT AND NOVEL IMAGING TECHNIQUES TO PREDICT OVARIAN CANCER RESECTABILITY

Chapter 5 Prediction of incomplete primary debulking surgery in patients with advanced ovarian cancer: An external validation study of three models using computed tomography. Gynecologic Oncology (2016);140:22-8

Chapter 6 Diagnostic value of PET/MRI for assessment of ovarian cancer spread: A feasibility study. [Submitted] 


\section{Part IIII ULTRASOUND AND MRI STRATEGIES FOR}

CHARACTERIZATION OF ADNEXAL MASSES

Chapter 7 Accuracy of Diffusion-weighted and conventional Magnetic Resonance Imaging for differentiation of adnexal masses: A Meta-analysis [Submitted]

Chapter 8 Investigating the performance and cost-effectiveness of the Simple Ultrasound-Based Rules compared to the Risk of Malignancy Index in the diagnosis of ovarian cancer (SUBSONiC-study): protocol of a prospective multicenter cohort study in The Netherlands BioMed Central Cancer (2015);15:482

Chapter 9 Simple rules, not so simple; The use of International Ovarian Tumor Analysis (IOTA) terminology and simple rules in experienced hands in a prospective multicenter cohort study. [Accepted, European Journal of Ultrasound] 137

Chapter 10 Discussion

Chapter 11 Summary 165

Chapter 12 Epilogue

Valorisatie 169

Dankwoord

Curriculum Vitae

List of publications

List of abbreviations 

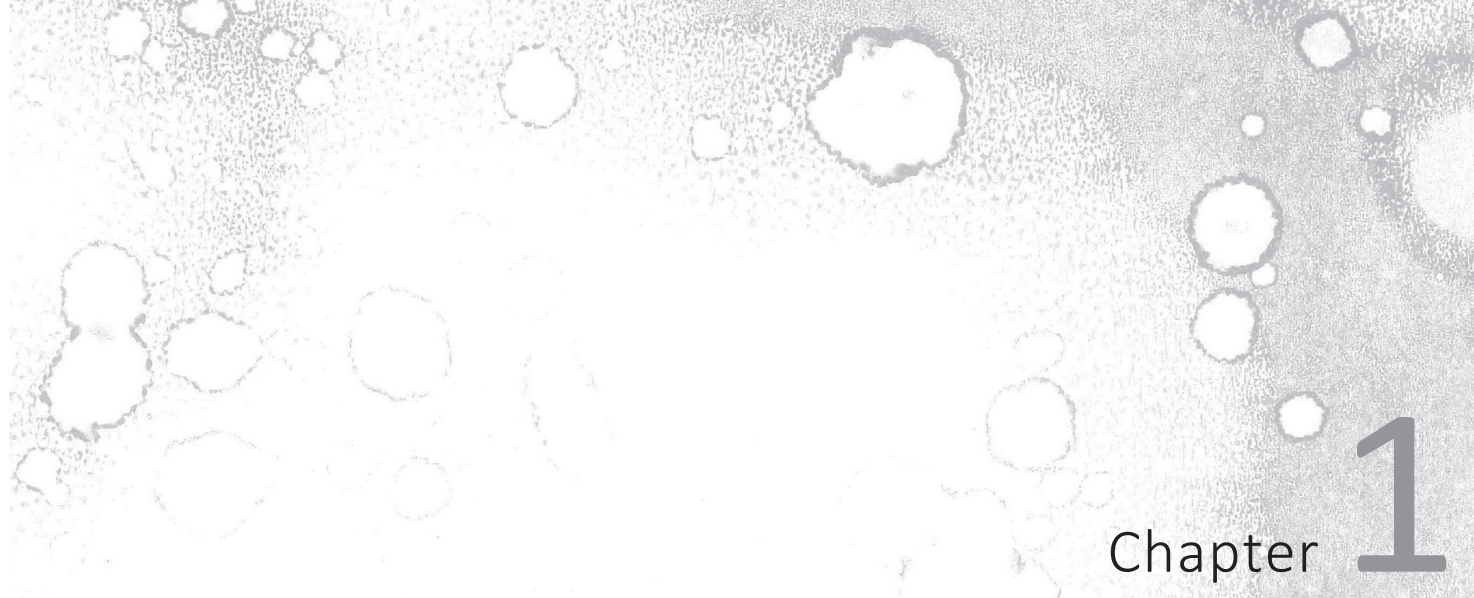

\section{General introduction}
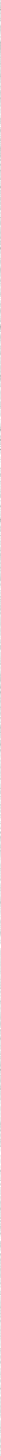

Epithelial ovarian cancer has the highest mortality of the gynaecological malignancies and claims 150.000 lives worldwide annually [1]. Five-year survival rates drop from 73$90 \%$ in early stage ovarian cancer to $17-39 \%$ in advanced stages [2; 3]. Between 60-70\% of patients are diagnosed with metastasized disease at first presentation due to the aggressive nature of ovarian cancer and paucity of symptoms [2]. The preferred treatment for patients with advanced ovarian cancer is upfront primary debulking surgery as it has proven to provide a survival benefit for patients with limited abdominal metastases [4]. Neoadjuvant chemotherapy complemented with interval debulking surgery provides a legitimate alternative with similar survival rates for patients who are inoperable or are estimated to have irresectable disease [4]. During the diagnostic and therapeutic process patients are subjected to various imaging methods to help in the diagnosis and characterization of their disease. This thesis investigates how we can use current and novel imaging strategies to optimally guide treatment decisions in order to improve ovarian cancer prognosis. In part I we look into possibilities to use existing standard computed tomography (CT) scans to find new (and possibly modifiable) predictors for survival. CT scans are used in standard clinical practice to detect ovarian tumour spread and to predict resectability. In part II we explore how accurate these CT scans and CT-based prediction models actually are and we consider two new imaging techniques for the prediction of resectability: diffusion-weighted magnetic resonance imaging (DW-MRI) and combined positron emission tomography magnetic resonance imaging (PET/MRI). Finally in part III we take a step back to where the diagnosis of ovarian cancer begins and find out what the value is of different ultrasound and magnetic resonance imaging (MRI) techniques in the differentiation between benign and malignant adnexal masses.

\section{PART I: CT-BASED ASSESSMENT OF SKELETAL MUSCLE LOSS AND OVARIAN CANCER SURVIVAL}

CT imaging is essential in the diagnostic work-up of ovarian cancer patients and is used by radiologists and gynaecologists worldwide to assess tumour extent and to estimate whether complete tumour resection can be achieved. Besides using CT to assess tumour locations, this imaging method can also be used to look beyond the tumour and focus on other patient characteristics. In this part of the thesis we have studied CT images to identify a potential predictor of ovarian cancer survival: sarcopenia, a severe loss of skeletal muscle mass. Sarcopenia is part of the cancer cachexia syndrome and the mechanisms behind it involve an interplay between reduced nutrient intake and abnormal metabolism incited by an excessive systemic inflammation and catabolic tumour-related mediators to which the host (i.e. the patient) is unable to respond properly [5; 6]. Inactive and malnourished patients will experience more muscle wasting and are are thought to have more complications when undergoing surgery $[7 ; 8]$. 
Perioperative malnutrition has also been associated with higher rates of suboptimal debulking surgery and thus a shorter survival [9]. In addition muscle loss by itself is associated with poor physical function, increased chemotherapy toxicity and longer recovery after surgery [10-12]. Cross-sectional CT analysis of muscle tissue on a single slice strongly correlates with total body muscle mass and has thus been widely adopted for characterization of cancer patients [13; 14]. Sarcopenia has been associated with adverse clinical outcomes and survival in patients with various malignancies [15-19] but studies concerning ovarian cancer patients are scarce. In this thesis we study the influence of sarcopenia on outcome in two different cohorts of patients with advanced ovarian cancer. In Chapter 2 we investigate the relationship between skeletal muscle mass and overall survival in patients treated with neoadjuvant chemotherapy. By measuring skeletal muscle mass both before and after chemotherapy we can assess muscle changes arising during chemotherapy within the individual patient. Secondly we want to evaluate whether a single measurement on CT can also identify sarcopenic patients as opposed to the measurement of muscle change. In this way, patients can be identified timely to make an early intervention possible. To investigate this we study a cohort of ovarian cancer patients undergoing primary debulking surgery in Chapter 3. Besides assessing the relationship between sarcopenia and survival, in this cohort we also assess the relationship between sarcopenia and surgical complications. The association between sarcopenia and postoperative complications is thus far unknown in ovarian cancer but has shown significance in gastro-intestinal cancers [20; 21]. As an alternative for measuring all skeletal muscle visible on CT to identify sarcopenia - as we do in Chapters 2 and 3 - one can opt to evaluate only the psoas muscle area which is an easier and less time-consuming method. In Chapter 4 we aim to find out whether two methods to assess the psoas area give a reliable representation of sarcopenia in ovarian cancer with the same accuracy as assessment of the total muscle area.

\section{PART II: CURRENT AND NOVEL IMAGING TECHNIQUES TO PREDICT OVARIAN CANCER RESECTABILITY}

Maximal cytoreduction to no residual tumour has been the mainstay of advanced ovarian cancer treatment for many years now since Griffiths et al. established the inverse relationship between the magnitude of residual disease and overall survival in 1975 [22]. The amount of residual tumour after cytoreductive surgery is still the most important prognostic factor in patients with advanced ovarian cancer [4; 23-25]. Incomplete resection has little beneficial effects on survival while it can cause substantial perioperative morbidity. Therefore surgery should be avoided when incomplete resection is expected. Neoadjuvant chemotherapy followed by interval debulking surgery provides a good treatment alternative when complete primary cytoreductive surgery is deemed not feasible [4; 26]. CT imaging is currently used to 
estimate surgical resectability but has low sensitivity and specificity in predicting whether or not an optimal resection (tumour residual $\leq 1 \mathrm{~cm}$ ) can be achieved [27]. Numerous CT-based prediction models have been developed in order to improve the selection of patients who are unlikely to benefit from primary debulking surgery [2838]. However, few of the developed models have been tested for external validity. Also, the multitude of combinations of predictors found to be associated with ovarian cancer resectability makes the reproducibility and clinical applicability of these models questionable. Because an accurate model could be clinically useful, we set out to externally validate three prospectively developed CT-based models to predict incomplete debulking surgery in Chapter 5. The models will be applied to our validation cohort and their accuracy will be tested when interpreted by different readers.

The limited accuracy of CT has in recent years led to the exploration of other imaging techniques such as MRI and positron emission tomography (PET) to evaluate ovarian cancer. MRI provides anatomic detail in staging of local tumour extent due to its high soft tissue resolution. Functional techniques such as diffusion weighted imaging (DWI) further enhance both local and distant lesion detection and characterisation [39]. PET imaging is commonly used in cancer imaging and uses molecular imaging technology to detect metabolically active tumour sites and adenopathy. In Chapter 6 we explore the feasibility of the whole-body fully integrated PET/MRI imaging system in the assessment of surgical resectability. PET/MRI combines anatomic MRI with functional PET and DWI within a single examination. Because the technique is still new, the most appropriate oncologic applications in which PET/MRI has clinical importance still have to be studied. The objective of this prospective study is to compare the diagnostic performance of PET/MRI and DW-MRI with CT in the assessment of ovarian cancer spread in women with advanced stage ovarian cancer undergoing primary or interval debulking surgery.

\section{PART III: ULTRASOUND AND MRI STRATEGIES FOR CHARACTERIZATION OF ADNEXAL MASSES}

Correct differentiation between a benign and malignant adnexal mass is essential for its further management. Benign lesions can be treated conservatively or with minimally invasive surgery whereas malignant lesions need more tailored treatment in specialized oncologic centres. The Risk of Malignancy Index (RMI) is currently the most used method to characterize an adnexal mass. This index incorporates five ultrasound variables, the menopausal status and serum CA-125 to predict the likelihood that an adnexal mass is malignant [40]. However, around $20 \%$ of patients with an adnexal mass are incorrectly diagnosed by means of the RMI and receive inappropriate treatment [41]. Nonetheless ultrasound is a non-invasive and accessible method that remains the primary diagnostic tool for characterization of adnexal masses. New imaging strategies 
involving ultrasound models have been developed to achieve a more accurate diagnosis. The International Ovarian Tumour Analysis (IOTA) group successfully developed ten ultrasound rules to characterize an adnexal mass: the simple ultrasoundbased rules (Simple Rules) [42]. By using the Simple Rules, $80 \%$ of adnexal masses can be characterized as benign or malignant. In the remaining $20 \%$ the Simple Rules are inconclusive and a secondary test is desired. The subjective assessment of an expert ultrasonographer in the case of an inconclusive result has shown to improve diagnostic accuracy [43]. Alternative techniques that can be consulted in complex cases involve MRI and DW-MRI, which adds functional information to help classify a malignant adnexal mass. In Chapter 7 we study the diagnostic performance of MRI and the added value of DW-MRI for differentiation between benign and malignant adnexal masses in an extensive meta-analysis of the existing literature. Additionally, to assess the diagnostic performance of ultrasound and MRI in the characterization of adnexal masses we propose a prospective multicentre study. In Chapter 8 we provide the study protocol of the SUBSONiC study which has the objective to assess the value of a twostep triage model comprised of ultrasound Simple Rules complemented with expert ultrasound or DW-MRI in case of an inconclusive result. Finally, one of the secondary objectives of the SUBSONiC study is to evaluate interobserver variability between the primary ultrasound and the expert ultrasound in the interpretation of the Simple Rules and subjective assessment. The interim results hereof are provided in Chapter 9 in which we analyse how well untrained examiners and expert examiners are able to classify adnexal masses with the Simple Rules.

\section{THESIS OBJECTIVES}

- To investigate the overall survival related to changes in skeletal muscle for patients with advanced ovarian cancer treated with neoadjuvant chemotherapy (Chapter 2).

- To investigate whether sarcopenia is associated with overall survival and surgical complications in patients with advanced ovarian cancer undergoing primary debulking surgery (Chapter 3$)$.

- To investigate whether assessment of psoas muscle area reflects total muscle area and can be used to assess sarcopenia in ovarian cancer patients (Chapter 4).

- To test the ability of three prospectively developed CT models to predict incomplete primary debulking surgery in patients with advanced ovarian cancer (Chapter 5).

- To investigate the diagnostic performance of PET/MRI in the assessment of ovarian cancer spread in patients undergoing debulking surgery (Chapter 6). 
- To evaluate the diagnostic accuracy of contrast-enhanced MRI and DW-MRI for differentiation between benign and malignant adnexal masses (Chapter 7).

- To test the hypothesis that Simple Rules complemented with expert ultrasound or DW-MRI in case of an inconclusive result, will have better diagnostic accuracy for the characterization of adnexal masses than the Risk of Malignancy Index (Chapter 8).

- To analyse how well untrained examiners are able to apply the IOTA terminology and Simple Rules (Chapter 9). 


\section{REFERENCES}

1 Ferlay J, Soerjomataram I, Ervik M et al GLOBOCAN 2012 v1.0, Cancer Incidence and Mortality Worldwide: IARC CancerBase No. 11, 2013.

2 Surveillance E, and End Results (SEER) Program SEER 9 Incidence \& U.S. Mortality Research Data (19732011). In: National Cancer Institute D, Surveillance Research Program, Surveillance Systems Branch, released April 2014, based on the November 2013 submission, (ed),

3 van Altena AM, Karim-Kos HE, de Vries E, Kruitwagen RF, Massuger LF, Kiemeney LA (2012) Trends in therapy and survival of advanced stage epithelial ovarian cancer patients in the Netherlands. Gynecol Oncol 125:649-654

4 Vergote I, Trope CG, Amant F et al (2010) Neoadjuvant chemotherapy or primary surgery in stage IIIC or IV ovarian cancer. N Engl J Med 363:943-953

5 Fearon K, Strasser F, Anker SD et al (2011) Definition and classification of cancer cachexia: an international consensus. Lancet Oncol 12:489-495

6 Fearon K, Arends J, Baracos V (2013) Understanding the mechanisms and treatment options in cancer cachexia. Nat Rev Clin Oncol 10:90-99

7 Kortebein P, Ferrando A, Lombeida J, Wolfe R, Evans WJ (2007) Effect of 10 days of bed rest on skeletal muscle in healthy older adults. JAMA 297:1772-1774

8 Schiesser M, Kirchhoff P, Muller MK, Schafer M, Clavien PA (2009) The correlation of nutrition risk index, nutrition risk score, and bioimpedance analysis with postoperative complications in patients undergoing gastrointestinal surgery. Surgery 145:519-526

9 Alphs HH, Zahurak ML, Bristow RE, Diaz-Montes TP (2006) Predictors of surgical outcome and survival among elderly women diagnosed with ovarian and primary peritoneal cancer. Gynecol Oncol 103:10481053

10 Baracos V, Kazemi-Bajestani SM (2013) Clinical outcomes related to muscle mass in humans with cancer and catabolic illnesses. Int J Biochem Cell Biol 45:2302-2308

11 Prado CM, Lieffers JR, Bowthorpe L, Baracos VE, Mourtzakis M, McCargar LJ (2013) Sarcopenia and physical function in overweight patients with advanced cancer. Can J Diet Pract Res 74:69-74

12 Prado CM, Baracos VE, McCargar $\sqcup$ et al (2009) Sarcopenia as a determinant of chemotherapy toxicity and time to tumor progression in metastatic breast cancer patients receiving capecitabine treatment. Clin Cancer Res 15:2920-2926

13 Mourtzakis M, Prado CM, Lieffers JR, Reiman T, McCargar LJ, Baracos VE (2008) A practical and precise approach to quantification of body composition in cancer patients using computed tomography images acquired during routine care. Appl Physiol Nutr Metab 33:997-1006

14 Ross R (2003) Advances in the application of imaging methods in applied and clinical physiology. Acta Diabetol 40 Suppl 1:S45-50

15 Fujiwara N, Nakagawa H, Kudo Y et al (2015) Sarcopenia, Intramuscular Fat Deposition, and Visceral Adiposity Independently Predict the Outcomes of Hepatocellular Carcinoma. J Hepatol. 10.1016/j.jhep.2015.02.031

16 Martin L, Birdsell L, Macdonald N et al (2013) Cancer cachexia in the age of obesity: skeletal muscle depletion is a powerful prognostic factor, independent of body mass index. J Clin Oncol 31:1539-1547

17 Psutka SP, Boorjian SA, Moynagh MR et al (2016) Decreased Skeletal Muscle Mass is Associated with an Increased Risk of Mortality after Radical Nephrectomy for Localized Renal Cell Cancer. J Urol 195:270276

18 Shachar SS, Williams GR, Muss HB, Nishijima TF (2016) Prognostic value of sarcopenia in adults with solid tumours: A meta-analysis and systematic review. Eur J Cancer 57:58-67

19 van Vledder MG, Levolger S, Ayez N, Verhoef C, Tran TC, Ijzermans JN (2012) Body composition and outcome in patients undergoing resection of colorectal liver metastases. Br J Surg 99:550-557 
20 Lieffers JR, Bathe OF, Fassbender K, Winget M, Baracos VE (2012) Sarcopenia is associated with postoperative infection and delayed recovery from colorectal cancer resection surgery. $\mathrm{Br} J$ Cancer 107:931-936

21 Fukuda Y, Yamamoto K, Hirao M et al (2015) Sarcopenia is associated with severe postoperative complications in elderly gastric cancer patients undergoing gastrectomy. Gastric Cancer. 10.1007/s10120-015-0546-4

22 Griffiths CT (1975) Surgical resection of tumor bulk in the primary treatment of ovarian carcinoma. Natl Cancer Inst Monogr 42:101-104

23 Chi DS, Eisenhauer EL, Lang J et al (2006) What is the optimal goal of primary cytoreductive surgery for bulky stage IIIC epithelial ovarian carcinoma (EOC)? Gynecol Oncol 103:559-564

24 du Bois A, Reuss A, Pujade-Lauraine E, Harter P, Ray-Coquard I, Pfisterer J (2009) Role of surgical outcome as prognostic factor in advanced epithelial ovarian cancer: a combined exploratory analysis of 3 prospectively randomized phase 3 multicenter trials: by the Arbeitsgemeinschaft Gynaekologische Onkologie Studiengruppe Ovarialkarzinom (AGO-OVAR) and the Groupe d'Investigateurs Nationaux Pour les Etudes des Cancers de I'Ovaire (GINECO). Cancer 115:1234-1244

25 Chang SJ, Hodeib M, Chang J, Bristow RE (2013) Survival impact of complete cytoreduction to no gross residual disease for advanced-stage ovarian cancer: a meta-analysis. Gynecol Oncol 130:493-498

26 Kehoe S, Hook J, Nankivell M et al (2015) Primary chemotherapy versus primary surgery for newly diagnosed advanced ovarian cancer (CHORUS): an open-label, randomised, controlled, non-inferiority trial. Lancet 386:249-257

27 Qayyum A, Coakley FV, Westphalen AC, Hricak H, Okuno WT, Powell B (2005) Role of CT and MR imaging in predicting optimal cytoreduction of newly diagnosed primary epithelial ovarian cancer. Gynecol Oncol 96:301-306

28 Bristow RE, Duska LR, Lambrou NC et al (2000) A model for predicting surgical outcome in patients with advanced ovarian carcinoma using computed tomography. Cancer 89:1532-1540

29 Dowdy SC, Mullany SA, Brandt KR, Huppert BJ, Cliby WA (2004) The utility of computed tomography scans in predicting suboptimal cytoreductive surgery in women with advanced ovarian carcinoma. Cancer 101:346-352

30 Ferrandina G, Sallustio G, Fagotti A et al (2009) Role of CT scan-based and clinical evaluation in the preoperative prediction of optimal cytoreduction in advanced ovarian cancer: a prospective trial. $\mathrm{Br} \mathrm{J}$ Cancer 101:1066-1073

31 Gerestein CG, Eijkemans MJ, Bakker J et al (2011) Nomogram for suboptimal cytoreduction at primary surgery for advanced stage ovarian cancer. Anticancer Res 31:4043-4049

32 Jung DC, Kang S, Kim MJ, Park SY, Kim HB (2010) Multidetector CT predictors of incomplete resection in primary cytoreduction of patients with advanced ovarian cancer. Eur Radiol 20:100-107

33 Jung DC, Kang S, Kim SC et al (2013) Use of complex surgical procedures, patterns of tumor spread, and CA-125 predicts a risk of incomplete cytoreduction: a Korean Gynecologic Oncology Group study (KGOG3022). Gynecol Oncol 131:336-340

34 Kim HJ, Choi CH, Lee YY et al (2014) Surgical outcome prediction in patients with advanced ovarian cancer using computed tomography scans and intraoperative findings. Taiwan J Obstet Gynecol 53:343347

35 MacKintosh ML, Rahim R, Rajashanker B et al (2014) CT scan does not predict optimal debulking in stage III-IV epithelial ovarian cancer: a multicentre validation study. J Obstet Gynaecol 34:424-428

36 Mousavi AS, Mazhari MM, Guilani MM, Ghaemmaghami F, Behtash N, Akhavan S (2010) Can primary optimal cytoreduction be predicted in advanced epithelial ovarian cancer preoperatively? World J Surg Oncol 8:11

37 Rutten MJ, van de Vrie R, Bruining A et al (2015) Predicting surgical outcome in patients with International Federation of Gynecology and Obstetrics stage III or IV ovarian cancer using computed tomography: a systematic review of prediction models. Int J Gynecol Cancer 25:407-415 


\section{Chapter 1}

38 Stashwick C, Post MD, Arruda JS et al (2011) Surgical risk score predicts suboptimal debulking or a major perioperative complication in patients with advanced epithelial ovarian, fallopian tube, or primary peritoneal cancer. Int J Gynecol Cancer 21:1422-1427

39 Michielsen K, Vergote I, Op de Beeck K et al (2014) Whole-body MRI with diffusion-weighted sequence for staging of patients with suspected ovarian cancer: a clinical feasibility study in comparison to CT and FDG-PET/CT. Eur Radiol 24:889-901

40 Tingulstad S, Hagen B, Skjeldestad FE, Halvorsen T, Nustad K, Onsrud M (1999) The risk-of-malignancy index to evaluate potential ovarian cancers in local hospitals. Obstet Gynecol 93:448-452

41 Kaijser J, Sayasneh A, Van Hoorde K et al (2014) Presurgical diagnosis of adnexal tumours using mathematical models and scoring systems: a systematic review and meta-analysis. Hum Reprod Update 20:449-462

42 Timmerman D, Testa AC, Bourne $T$ et al (2008) Simple ultrasound-based rules for the diagnosis of ovarian cancer. Ultrasound Obstet Gynecol 31:681-690

43 Meys EM, Kaijser J, Kruitwagen RF et al (2016) Subjective assessment versus ultrasound models to diagnose ovarian cancer: A systematic review and meta-analysis. Eur J Cancer 58:17-29 


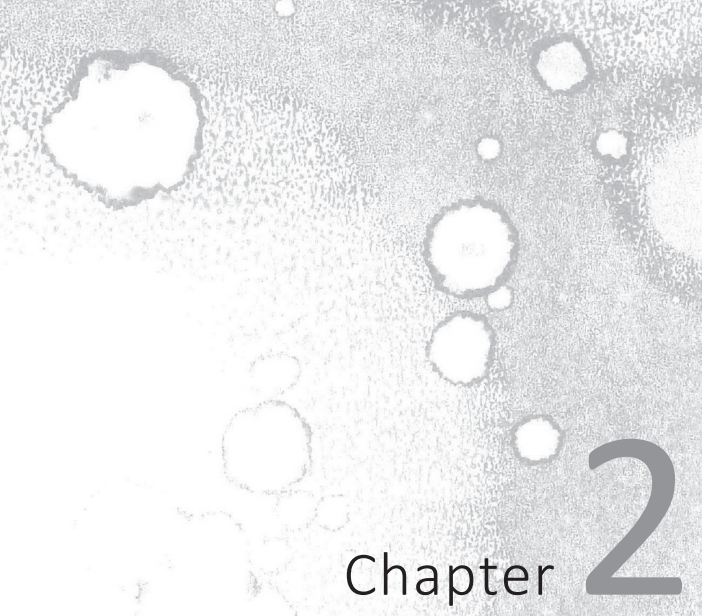

Loss of skeletal muscle during neoadjuvant chemotherapy is related to decreased survival in ovarian cancer patients

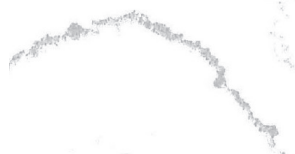




\section{Chapter 2}

\section{ABSTRACT}

Background: Malnutrition, weight loss and muscle wasting (sarcopenia) are common amongst women with advanced ovarian cancer and have been associated with adverse clinical outcomes and survival. Our objective is to investigate overall survival (OS) related to changes in skeletal muscle (SM) for patients with advanced ovarian cancer treated with neoadjuvant chemotherapy and interval debulking.

Methods: Ovarian cancer patients $(n=123)$ treated with neoadjuvant chemotherapy and interval debulking in the area of Maastricht (the Netherlands) between 2000 and 2014 were included retrospectively. Surface areas of SM and adipose tissue were defined on computed tomography at the level of the third lumbar vertebra. Low SM at baseline and SM changes during chemotherapy were compared with Kaplan Meier curves and Cox-regression models were applied to test predictors of OS.

Results: Median OS for patients who lost SM ( $n=83$ ) was $916 \pm 99$ days, which was significantly different from median OS for patients who maintained or gained SM $(n=40)$, which was $1431 \pm 470$ days $(p=0.004)$. Loss of SM was also a significant predictor of OS in multivariable Cox-regression analysis (hazard ratio 1.773 (95\% Cl: 1.018-3.088), $p=0.043)$. Low baseline SM did not influence survival.

Conclusions: Patients with ovarian cancer have a worse survival when they lose SM during neoadjuvant chemotherapy. Evaluation of low SM at a specific time point is not prognostic for OS. External and prospective validation of these findings is imperative. Nutritional, pharmacological and/or physical intervention studies are necessary to establish whether SM impairment can be prevented to prolong ovarian cancer survival. 


\section{INTRODUCTION}

Although survival rates have improved over the past decades, ovarian cancer still has the highest mortality of the gynaecological malignancies [1]. Ovarian cancer is responsible for 150.000 deaths worldwide annually and 5-year survival in Europe and the USA is 38-45\% [1-3]. Prognostic factors that have been identified for women diagnosed with ovarian cancer are age, performance status, histologic tumor type, tumor stage (Fédération Internationale de Gynécologie et d'Obstétrique, FIGO stage) and preoperative tumor load [4; 5]. However, complete resection of macroscopic tumor at cytoreductive surgery has been found to be the most important prognostic factor and it is vital that surgery is always aimed at achieving this goal [4; 5]. Primary debulking is the preferred treatment for patients with advanced ovarian cancer. When primary debulking is not possible because the patient's physical condition does not allow it or it is estimated that complete tumor resection cannot be accomplished, neoadjuvant chemotherapy followed by interval debulking provides an alternative treatment option with comparable survival rates [5].

Cancer cachexia, a syndrome of involuntary weight loss and muscle wasting, is common amongst women with advanced ovarian cancer and has also been associated with adverse clinical outcomes and survival [6]. However, weight loss is a poor indicator of disease status in ovarian cancer considering the fact that it is frequently not apparent due to growing volumes of ascites, oedema or the tumour itself including its metastases. In like manner, other changes such as muscle wasting or accumulation of adipose tissue in different compartments of the body remain indiscernible to the beholder when bodyweight alone is evaluated. In recent years, the understanding of cancer-related weight loss has therefore guided research to the study of body composition features rather than bodyweight alone. Computed tomography (CT) has been extensively studied and applied in this field and has the advantage that scans are often readily available for cancer patients. CT imaging enables precise quantification of skeletal muscle mass and different adipose depots on a single slice, which can be used to estimate total body muscle and fat mass [7; 8]. Cross-sectional analysis of tissue at the level of the third lumbar vertebra (L3) strongly correlates with total body adipose and muscle mass and has thus been widely adopted for characterisation of cancer patients $[7 ; 9 ; 10]$. Among the compartments that can be distinguished on CT are skeletal muscle (SM), intramuscular adipose tissue (IMAT), visceral adipose tissue (VAT) and subcutaneous adipose tissue (SAT).

Severe loss of skeletal muscle mass - known as sarcopenia - is associated with poor survival in patients with various types of cancer [11-14]. Adverse effects are also seen from redistribution of adipose tissue from subcutaneous depots to storage sites in skeletal muscle and the abdominal cavity. [11; 15; 16]. Studies concerning body composition and survival in patients with ovarian cancer are scarce. In a retrospective study of advanced ovarian cancer patients undergoing primary debulking surgery, SM 
and VAT were not predictive of survival but low combined SAT+IMAT was associated with worse overall survival (OS) [17]. These results have not been confirmed by others. Changes in body composition in patients undergoing neoadjuvant or palliative chemotherapy have been evaluated in oesophageal, gastric, pancreatic and lung cancer. [18-21]. These studies have included relatively small numbers of patients, which makes it difficult to assess the validity of their findings. The impact of neoadjuvant chemotherapy on muscle mass in ovarian cancer patients has not been investigated.

This study aims to investigate OS in patients with ovarian cancer related to the changes in skeletal muscle mass and body composition arising during neoadjuvant chemotherapy.

\section{METHODS}

\section{Eligible patients}

This study has been approved by the local Medical Ethics Committee and has been performed in accordance with the ethical standards laid down in the 1964 Declaration of Helsinki. The Medical Ethics Committee waived the requirement for obtaining informed consent.

A retrospective study was performed including patients with advanced ovarian cancer (FIGO 2013 stage IIB-IV) who were treated with neoadjuvant chemotherapy prior to interval debulking in the Maastricht University Medical Centre (Maastricht, the Netherlands) between January 2000 and June 2014. Subjects were eligible for inclusion when the following criteria were met: 1) a routine abdominal CT scan was performed before the start of neoadjuvant chemotherapy and a second abdominal CT scan before interval debulking (typically after 3-4 cycles of chemotherapy), 2) both CT scans were of sufficient quality to perform accurate measurements of tissue area, and 3) sufficient relevant clinical data could be retrieved from the patients' file. Overall survival was computed from the date of the initial CT scan up to the date of death from any cause. The CT date was chosen instead of the date of diagnosis because this date could not be retrieved for all patients. For patients who were still alive at the time of analysis, a fixed date was set for data collection and all patients were censored at this date, which was at least 6 months after the last included subject was diagnosed.

The following clinical characteristics were recorded: age, FIGO stage, length, weight, weight loss preceding diagnosis, number of chemotherapy cycles, days between CT scans, percentage weight loss during chemotherapy and surgical outcome. Age was evaluated at the time of the initial CT scan and categorised into $\leq 60$ years, 61-70 years and $>70$ years. Reported weight and length were used to calculate body mass index (BMI) in $\mathrm{kg} / \mathrm{m}^{2}$. Patients were assigned to BMI categories established by the World Health Organization (WHO): BMI <18.5 = underweight, BMI 18.5-24.9 = normal weight, 
BMI 25-29.9 = overweight and BMI $\geq 30$ = obese [22]. The outcome of interval debulking was categorised into complete (no visible evidence of macroscopic residual disease), optimal (macroscopic residual disease $\leq 1 \mathrm{~cm}$ ) or incomplete (macroscopic residual disease $>1 \mathrm{~cm}$ ).

\section{Body composition measurements}

For each CT scan a single axial slice at the level of L3 was selected. Image analysis software, SliceOmatic v5.0 (Tomovision, Montreal, QC, Canada), was used to demarcate SM, IMAT, VAT and SAT according to predefined validated boundaries based on the number of Hounsfield Units (HU). An example of how tissues were measured with SliceOmatic software is shown in Figure 1. The following thresholds were applied: -29 to $+150 \mathrm{HU}$ for SM, -190 to -30 HU for IMAT and SAT and -150 to -50 HU for VAT. A single assessor who was trained in the anatomy of the specific tissues of interest evaluated all scans subsequently and the surface areas in $\mathrm{cm}^{2}$ were quantified automatically based on the demarcations. The assessor was not aware of the outcome of the patients. Total adipose tissue (TAT) was computed by summating IMAT, VAT and SAT. The surface area of SM was normalised for stature to compute the skeletal muscle index (SMI) in $\mathrm{cm}^{2} / \mathrm{m}^{2}$. The median SMI at baseline from our own population was used to divide patients in a low muscle mass group (SMI below median) and a high muscle mass group (SMI equal to or above median).

Changes in surface area between CT scans were expressed as a percentage. This percentage change was divided by the number of days between scans and multiplied by 100 days to provide a standard measure for all patients (\% change per 100 days). A measurement error of $2 \%$ was adopted based on previously reported accuracy of CT for muscle and fat tissue analysis [7]. Changes between $-2 \%$ and $+2 \%$ were thus considered "maintenance of tissue". Finally, changes were dichotomised into loss of tissue ( $>2 \%$ decrease per 100 days) and maintenance/gain of tissue (any increase or $\leq 2 \%$ decrease). For practical reasons the term "gain of tissue" will hereafter be used to describe both maintenance and gain of tissue.

\section{Statistical analysis}

The mean changes in muscle and adipose tissue were analysed with paired T-tests and the mean percentage change per 100 days was calculated for SM, IMAT, VAT, SAT and TAT. Survival curves were computed using Kaplan-Meier estimates with Log-Rank tests. "Low baseline SMI" was compared with "high baseline SMI" and "loss of SM" was compared with "gain of SM". Baseline characteristics were analysed with independent t-tests for continuous variables and chi-squared or Fisher's exact tests for categorical variables. Statistical significance for all comparative tests was determined at $p<0.05$. 


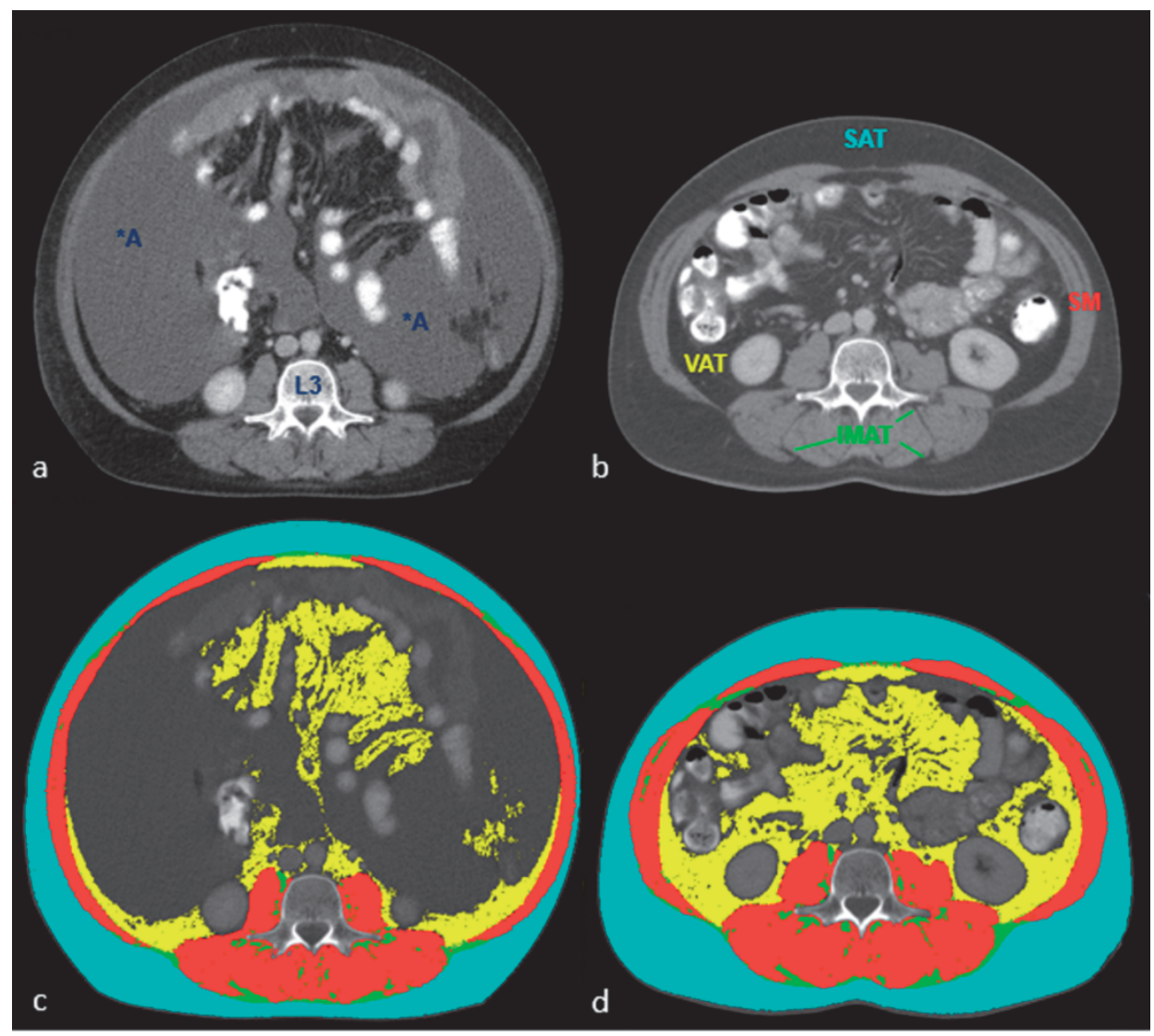

Fig. 1 Body composition analysis with SliceOmatic ${ }^{\circledR}$

Example of CT scans pre $(a, c)$ and post $(b, d)$ chemotherapy in a 46-year old patient with FIGO stage IV ovarian cancer. Increases in SM, IMAT, VAT and SAT were measured with SliceOmatic v5.0 (Tomovision, Montreal, QC, Canada). NB: The increase in VAT is accompanied by a reduction of ascites

$A^{*}=$ ascites, $L 3=$ third lumbar vertebra, $S M=$ skeletal muscle (red), IMAT = intramuscular adipose tissue (green), VAT = visceral adipose tissue (yellow), SAT = subcutaneous adipose tissue (teal).

Univariable and multivariable proportional hazards Cox-regression models were applied to test predictors of OS and calculate individual hazard ratio's (HR) with 95\% confidence intervals $(95 \% \mathrm{Cl})$. Clinical variables and body composition parameters were initially tested as effect modifiers in a univariable model at a significance level of $10 \%$. All significant variables were then tested together in a multivariable model in which a significance level of $5 \%$ was applied. The continuous variables that were tested were age, BMI (pre and post chemotherapy), weight loss during chemotherapy, weight loss preceding diagnosis and number of cycles of chemotherapy. Age and BMI were also assessed categorically. The categorical variables of main interest were changes in SM, IMAT, VAT, SAT and TAT during chemotherapy where loss of tissue was compared with gain of tissue. The remaining categorical variables tested were low SMI at baseline and 
after chemotherapy (yes or no), FIGO stage IV (in comparison with FIGO stages II and III combined), presence of ascites (yes or no), and complete interval debulking (in comparison with optimal and incomplete interval debulking). All subjects were analysed and missing data were indicated separately when applicable. All analyses were performed with the statistical software package SPSS v20.0 (IBM Corp, Chicago, IL).

\section{RESULTS}

We identified 566 patients that were treated for ovarian cancer in the Maastricht University Medical Centre between January 2000 and June 2014 (Figure 2). After exclusion of 436 patients (patients with non-advanced disease, patients without CT examinations, patients who only underwent primary debulking and/or patients without sufficient clinical data), 130 patients were evaluated with CT measurements. Another 7 patients were excluded either due to missing CT scans pre or post chemotherapy or due to insufficient quality of the scans. Final analysis was conducted on 123 patients with 246 CT scans. Baseline characteristics for the included subjects are presented in Table 1. At the time of censoring, 52 out of 123 patients (42\%) were still alive. Follow-up for this group was at least 6 months and ranged from 231 days to 3850 days with a median of 681 days.

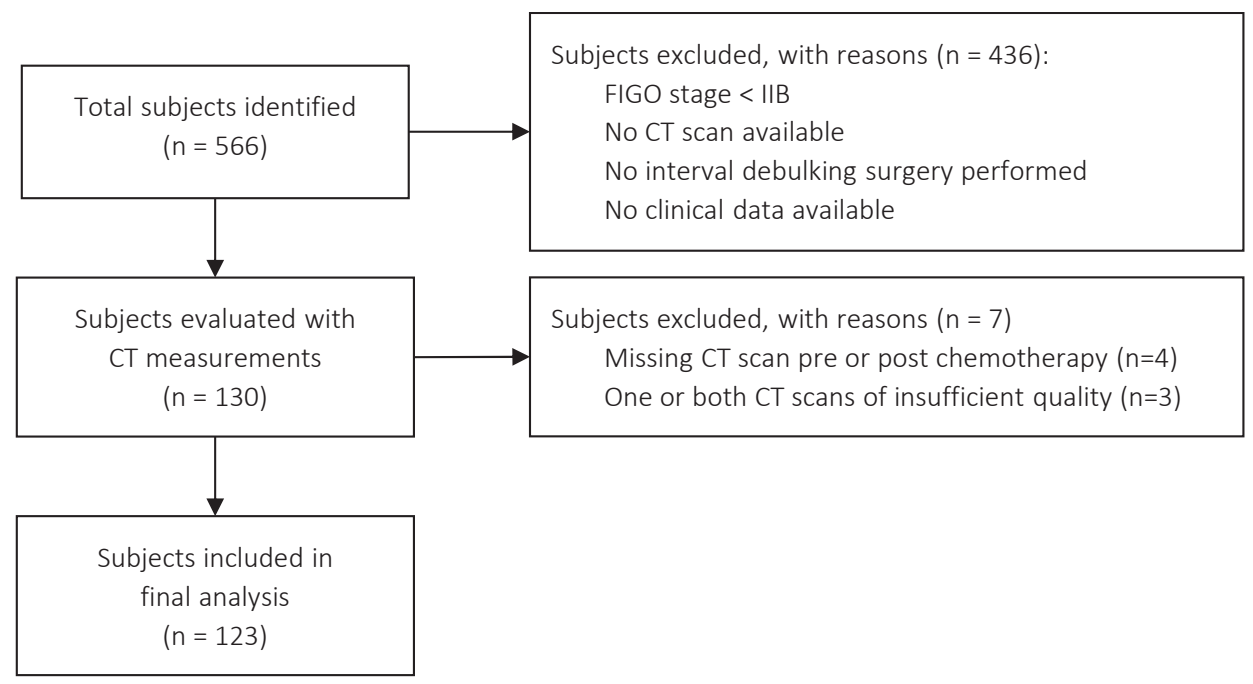

Fig. 2 Flow diagram 


\section{Chapter 2}

Table 1 Baseline Characteristics

\begin{tabular}{|c|c|c|c|c|}
\hline & & All patients $(n=123)$ & SM Loss $(n=83)$ & SM Gain $(n=40)$ \\
\hline Age in years & mean \pm SE (range) & $66.5 \pm 0.8(39-86)$ & $67.6 \pm 1.0(48-86)$ & $64.2 \pm 1.6(39-82)$ \\
\hline$\leq 60$ years & $\mathrm{n}(\%)$ & $33(26.8)$ & $18(21.7)$ & $15(37.5)$ \\
\hline $61-70$ years & $\mathrm{n}(\%)$ & $46(37.4)$ & $31(37.3)$ & $15(37.5)$ \\
\hline$>70$ years & $\mathrm{n}(\%)$ & $44(35.8)$ & $34(41.0)$ & $10(25.0)$ \\
\hline \multicolumn{5}{|l|}{ FIGO stage } \\
\hline II & $\mathrm{n}(\%)$ & $2(1.6)$ & $2(2.4)$ & $0(0.0)$ \\
\hline III & $\mathrm{n}(\%)$ & $79(64.2)$ & $53(63.9)$ & $26(65.0)$ \\
\hline IV & $\mathrm{n}(\%)$ & $42(34.1)$ & $28(33.7)$ & $14(35.0)$ \\
\hline $\mathrm{BMI}$ in $\mathrm{kg} / \mathrm{m} 2 \mathrm{PRE}$ chemotherapy & mean \pm SE & $25.9 \pm 0.5$ & $26.6 \pm 0.5$ & $24.5 \pm 0.9$ \\
\hline Underweight (BMI < 18.5) & $\mathrm{n}(\%)$ & $2(1.6)$ & $1(1.2)$ & $1(2.5)$ \\
\hline Normal weight (BMI 18.5-24.9) & $\mathrm{n}(\%)$ & $31(25.2)$ & $17(20.5)$ & $14(35.0)$ \\
\hline Overweight (BMI 25.0-29.9) & $\mathrm{n}(\%)$ & $34(27.6)$ & $25(30.1)$ & $9(22.5)$ \\
\hline Obese $(\mathrm{BMI}>30.0)$ & $\mathrm{n}(\%)$ & $10(8.1)$ & $8(9.6)$ & $2(5.0)$ \\
\hline Missing values & $\mathrm{n}(\%)$ & $46(37.4)$ & $32(38.6)$ & $14(35.0)$ \\
\hline BMI in kg/m2 POST chemotherapy & mean $\pm \mathrm{SE}$ & $25.0 \pm 0.3$ & $25.5 \pm 0.4$ & $24.1 \pm 0.6$ \\
\hline Underweight $(\mathrm{BMI}<18.5)$ & $\mathrm{n}(\%)$ & $1(0.8)$ & $0(0.0)$ & $1(2.5)$ \\
\hline Normal weight (BMI 18.5-24.9) & $\mathrm{n}(\%)$ & $61(49.6)$ & $39(47.0)$ & $22(55.0)$ \\
\hline Overweight (BMI 25.0-29.9) & $\mathrm{n}(\%)$ & $43(35.0)$ & $31(37.3)$ & $12(30.0)$ \\
\hline Obese $(\mathrm{BMI}>30.0)$ & $\mathrm{n}(\%)$ & $10(8.1)$ & $8(9.6)$ & $2(5.0)$ \\
\hline Missing values & $\mathrm{n}(\%)$ & $8(6.5)$ & $5(6.0)$ & $3(7.5)$ \\
\hline Weight loss preceding diagnosis in $\mathrm{kg}^{\mathrm{a}}$ & mean $\pm S E$ & $-3.7 \pm 0.6$ & $-3.7 \pm 0.8$ & $-3.7 \pm 1.0$ \\
\hline $\mathrm{SMI}$ at baseline in $\mathrm{cm}^{2} / \mathrm{m}^{2}$ & mean $\pm S E$ & $41.7 \pm 0.5$ & $42.8 \pm 0.6^{c}$ & $39.3 \pm 0.9^{c}$ \\
\hline Low baseline SMI (SMI < 41.5) & $\mathrm{n}(\%)$ & $62(50.4)$ & $34(41.0)^{\mathrm{c}}$ & $28(70.0)^{\mathrm{c}}$ \\
\hline SMI after chemotherapy in $\mathrm{cm}^{2} / \mathrm{m}^{2}$ & mean $\pm \mathrm{SE}$ & $39.8 \pm 0.5$ & $39.3 \pm 0.5$ & $40.7 \pm 0.9$ \\
\hline $\begin{array}{l}\text { Low SMI after chemotherapy (SMI < } \\
41.5)\end{array}$ & $\mathrm{n}(\%)$ & $80(65.0)$ & $56(67.5)$ & $24(60.0)$ \\
\hline Cycles of neoadjuvant chemotherapy & mean \pm SE & $3.43 \pm 0.1$ & $3.5 \pm 0.1$ & $3.4 \pm 0.1$ \\
\hline 3 & $\mathrm{n}(\%)$ & $87(70.7)$ & $58(69.9)$ & $29(72.5)$ \\
\hline 4 & $\mathrm{n}(\%)$ & $27(22.0)$ & $19(22.9)$ & $8(20)$ \\
\hline$\geq 5$ & $\mathrm{n}(\%)$ & $9(7.3)$ & $6(7.2)$ & $3(7.5)$ \\
\hline Days between CT scans & mean $\pm \mathrm{SE}$ & $84 \pm 1.77$ & $84 \pm 2.2$ & $84 \pm 3.0$ \\
\hline $\begin{array}{l}\text { Percentage weight loss during } \\
\text { chemotherapy }^{b}\end{array}$ & mean $\% \pm \mathrm{SE}$ & $-3.6 \pm 0.8$ & $-4.8 \pm 1.0^{c}$ & $-1.4 \pm 1.1^{\mathrm{c}}$ \\
\hline \multicolumn{5}{|l|}{ Outcome interval debulking } \\
\hline Complete & $\mathrm{n}(\%)$ & $56(45.5)$ & $34(41.0)$ & $22(55.0)$ \\
\hline Optimal & $\mathrm{n}(\%)$ & $42(34.1)$ & $31(37.3)$ & $11(27.5)$ \\
\hline Incomplete & $\mathrm{n}(\%)$ & $25(20.3)$ & $18(21.7)$ & $7(17.5)$ \\
\hline Presence of ascites & n (\%) & $67(54.5)$ & $43(51.8)$ & $24(60.0)$ \\
\hline OS in days & median \pm SD & $986 \pm 111$ & $916 \pm 99^{c}$ & $1431 \pm 470^{c}$ \\
\hline
\end{tabular}

$\mathrm{SE}=$ standard error of the mean, $\mathrm{SM}=$ skeletal muscle, $\mathrm{BMI}=$ Body Mass Index, $\mathrm{SMI}=$ skeletal muscle index, $\mathrm{CT}=\mathrm{computed}$ tomography, OS = overall survival.

${ }^{a}$ Missing values: 58/123 missing

${ }^{b}$ Missing values: 53/123 missing.

${ }^{\mathrm{c}}$ Significant differences between SM Loss \& SM Gain ( $<<0.05$, independent-samples T-test or chi-squared test). 


\section{Body composition at baseline}

Mean body composition measurements at baseline are shown in table 2. Median SMI at baseline was $41.5 \mathrm{~cm}^{2} / \mathrm{m}^{2}$, which was used as cut-off to define high and low baseline $\mathrm{SMI}$ in the study population.

Subjects with high versus low baseline SMI did not show significant differences in OS with a p-value of 0.613 in Kaplan Meier analysis (Figure 3).

Table 2 Body composition changes during neoadjuvant chemotherapy

\begin{tabular}{llll}
\hline & $\begin{array}{l}\text { Baseline L3 area in } \mathrm{cm}^{2} \\
(\text { median } \pm \text { SD) }\end{array}$ & $\begin{array}{l}\text { Change in L3 area in } \mathrm{cm}^{2} \\
(\text { median } \pm \mathrm{SD})\end{array}$ & $\begin{array}{l}\text { Rate of change in L3 area in \%/100 days } \\
\text { (median } \pm \text { SD) }\end{array}$ \\
\hline SM & $109.1 \pm 14.2$ & $-4.5 \pm 8.3^{*}$ & $-5.2 \pm 9.8$ \\
IMAT & $14.2 \pm 10.1$ & $+0.7 \pm 3.8^{* *}$ & $+5.6 \pm 34.7$ \\
VAT & $66.2 \pm 58.5$ & $-0.6 \pm 21.1^{* *}$ & $-0.8 \pm 56.3$ \\
SAT & $181.5 \pm 87.4$ & $-11.2 \pm 39.0^{*}$ & $-6.1 \pm 36.4$ \\
TAT & $266.0 \pm 140.0$ & $-10.9 \pm 51.3^{*}$ & $-4.5 \pm 34.1$ \\
\hline
\end{tabular}

$\mathrm{SD}=$ standard deviation, $\mathrm{SM}=$ skeletal muscle, IMAT = intramuscular adipose tissue, $\mathrm{VAT}=$ visceral adipose tissue, SAT = subcutaneous adipose tissue, TAT = total adipose tissue.

$* / * *$ indicate significant changes in L3 area measurements between scan 1 and $2(* p<0,001, * * p<0,05$ using Paired-Samples T-Test).

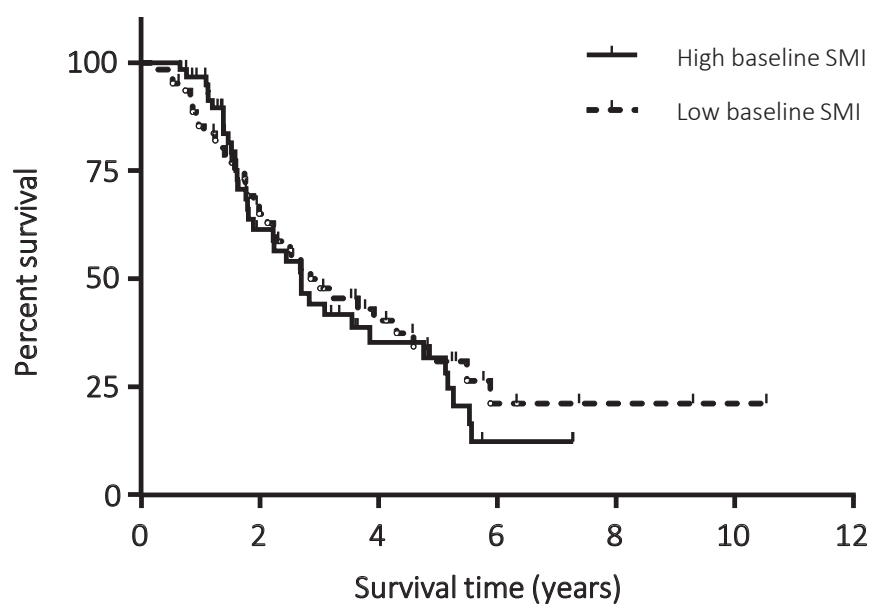

\# of subjects at risk

$\begin{array}{llll} & 0 y r & 2 y r & 5 y r \\ \text { Low baseline SMI } & 62 & 31 & 9 \\ \text { High baseline SMI } & 61 & 25 & 9\end{array}$

Fig. 3 Survival proportions: Baseline skeletal muscle index

Kaplan Meier curve comparing overall survival between high baseline SMI and low baseline SMI, p-value = 0.613

$S M I=$ skeletal muscle index 


\section{Body composition changes during neoadjuvant chemotherapy}

Mean body composition changes after neoadjuvant chemotherapy are shown in table 2 . Treatment with neoadjuvant chemotherapy resulted in significant decreases in mean SM, VAT, SAT and TAT as well as a significant increase in IMAT. Mean decrease in SM for this period was $5.2 \%$ per 100 days.

Subjects who were able to maintain or gain SM during chemotherapy had an increased OS in Kaplan Meier analysis in comparison to patients who lost SM ( $p=0.004$, Figure 4). This difference in survival was most prominent from 2 years after start of therapy onwards. To assess other differences between patients with gain or loss of muscle mass, baseline characteristics were presented separately for these groups in table 1. Besides a significant difference in OS as already noted in Kaplan Meier analysis, only 2 other variables showed differences between both groups. Mean SMI at baseline was significantly lower in the group of patients who could increase SM and low baseline SMI was present in $70 \%$ of these patients compared to $41 \%$ of patients who experienced muscle loss during chemotherapy $(p=0.003)$. Furthermore, patients who lost SM during chemotherapy also lost more weight in general between scans $(4.8 \%$ weight loss versus $1.4 \%$ weight loss, $\mathrm{p}=0.043$ ).

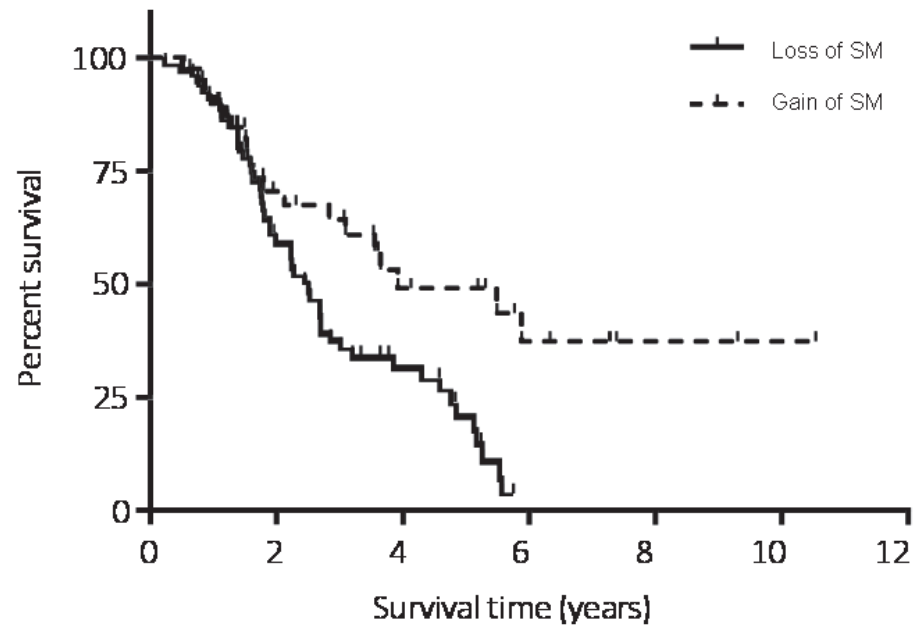

$\#$ of subjects at risk

$\begin{array}{llll} & 0 y r & 2 y r & 5 y r \\ \text { Loss of SM } & 83 & 33 & 7 \\ \text { Gain of SM } & 40 & 23 & 11\end{array}$

Fig. 4 Survival proportions: Changes in skeletal muscle Kaplan Meier curve comparing overall survival between loss of skeletal muscle (> 2\% decrease per 100 days) and maintenance or gain of skeletal muscle (any increase or $\leq 2 \%$ decrease per 100 days), $p$-value $=0.004$ SM = skeletal muscle 
Finally, we tested whether our variable of interest - change in SM - held significance in a Cox-regression model under influence of other potential predictors of OS. Only four variables were significant at the level of $10 \%$ in the univariable model and were included in the multivariable model: 1) age $>70$ years, 2) complete interval debulking, 3) loss of SM and 4) loss of VAT (Table 3). Age > 70 years did not retain significance in the multivariable model. Completeness of interval debulking had a positive outcome on OS with a HR of $0.49(p=0.005)$. Loss of SM and loss of VAT were also significant in the multivariable model and influenced OS negatively with HR's of 1.77 and 1.83 respectively.

Table 3 Univariable and multivariable Cox-regression analyses

\begin{tabular}{|c|c|c|c|c|}
\hline \multirow[b]{2}{*}{ Variables } & \multicolumn{2}{|l|}{ Univariable analysis } & \multicolumn{2}{|l|}{ Multivariable analysis } \\
\hline & $\mathrm{HR}(95 \% \mathrm{Cl})$ & p-value & $\mathrm{HR}(95 \% \mathrm{Cl})$ & p-value \\
\hline Age at diagnosis & $1.020(0.993-1.048)$ & 0.144 & & \\
\hline Age at diagnosis $\leq 60$ years & $0.913(0.547-1.523)$ & 0.726 & & \\
\hline Age at diagnosis $>70$ years & $1.616(0.999-2.616)$ & $0.051^{\mathrm{e}}$ & $1.274(0.780-2.080)$ & 0.333 \\
\hline BMI pre chemotherapy ${ }^{\mathrm{a}}$ & $1.027(0.963-1.096)$ & 0.413 & & \\
\hline BMI post chemotherapy ${ }^{b}$ & $0.998(0.931-1.070)$ & 0.950 & & \\
\hline Obesity pre chemotherapy $(\mathrm{BMI}>30)^{\mathrm{a}}$ & $1.099(0.431-2.802)$ & 0.843 & & \\
\hline Obesity post chemotherapy $(\mathrm{BMI}>30)^{\mathrm{b}}$ & $0.893(0.323-2.468)$ & 0.828 & & \\
\hline Percentage weight loss between scans ${ }^{c}$ & $0.968(0.924-1.015)$ & 0.180 & & \\
\hline Weight loss preceding diagnosis $^{d}$ & $1.005(0.934-1.080)$ & 0.903 & & \\
\hline FIGO stage IV & $1.480(0.918-2.387)$ & 0.108 & & \\
\hline Cycles of chemotherapy & $1.031(0.798-1.332)$ & 0.814 & & \\
\hline Complete interval debulking & $0.452(0.277-0.737)$ & $0.001^{f}$ & $0.488(0.296-0.806)$ & $0.005^{f}$ \\
\hline Loss of SM during chemotherapy & $2.218(1.280-3.844)$ & $0.005^{f}$ & $1.773(1.018-3.088)$ & $0.043^{f}$ \\
\hline Loss of IMAT during chemotherapy & $1.346(0.837-2.166)$ & 0.220 & & \\
\hline Loss of VAT during chemotherapy & $1.854(1.158-2.970)$ & $0.010^{f}$ & $1.828(1.131-2.952)$ & $0.014^{f}$ \\
\hline Loss of SAT during chemotherapy & $0.890(0.553-1.432)$ & 0.630 & & \\
\hline Loss of TAT during chemotherapy & $1.411(0.878-2.268)$ & 0.155 & & \\
\hline Low baseline SMI & $0.887(0.556-1.414)$ & 0.613 & & \\
\hline Low SMI after chemotherapy & $1.054(0.649-1.710)$ & 0.832 & & \\
\hline Presence of ascites at baseline & $1.111(0.689-1.794)$ & 0.665 & & \\
\hline
\end{tabular}

$\mathrm{HR}=$ hazard ratio, $\mathrm{Cl}=$ confidence interval, $\mathrm{BMI}=$ Body Mass Index, SM = skeletal muscle, IMAT = intramuscular adipose tissue, VAT = visceral adipose tissue, SAT = subcutaneous adipose tissue, TAT = total adipose tissue.

${ }^{a}$ Missing data for 46 patients, analysis was carried out with available data from 77 patients.

${ }^{\mathrm{b}}$ Missing data for 8 patients, analysis was carried out with available data from 115 patients.

${ }^{\mathrm{c}}$ Missing data for 53 patients, analysis was carried out with available data from 70 patients.

${ }^{d}$ Missing data for 58 patients, analysis was carried out with available data from 65 patients.

e P-value $<0.1$

${ }^{f}$ P-value $<0.05$. 


\section{DISCUSSION}

\section{Key findings}

Our goal was to investigate whether changes in skeletal muscle mass occur in ovarian cancer patients undergoing neoadjuvant chemotherapy and if so, whether these changes have an impact on survival. We found that women that maintained or gained SM during neoadjuvant treatment had a better prognosis than women that lost SM.

Loss of SM during chemotherapy and a shorter OS are closely related according to our findings but the direct causality of this relationship is unclear. Loss of SM is one of the hallmarks of cancer cachexia. The increased amount of weight loss in the muscle loss group suggests that these patients suffered from a higher degree of cachexia. The metabolic and inflammatory changes associated with cachexia together with the decreased muscle nitrogen reserves could explain the poor prognosis in this group [23]. Inactive and malnourished patients will experience more muscle wasting and are more prone to have complications when undergoing surgery [24; 25]. Reasons why patients are unable to maintain a healthy diet or physical activity are diverse. Patients with a good responding tumor might feel better compared to patients with bulky, poorresponding tumors. Perioperative malnutrition has also been associated with higher rates of suboptimal debulking surgery and thus a shorter survival in a study of older women diagnosed with ovarian cancer [26]. In addition, muscle loss by itself is associated with poor physical function, increased chemotherapy toxicity, and longer recovery after surgery [27-29]. Finally, the presence of comorbidities and old age could also be detrimental. However, age did not reach statistical significance in our multivariable Cox-regression survival model .

Interestingly, decrease in SM over time was an important prognostic factor for OS while low SM at a specific time point was not. We measured the SMI at baseline and after chemotherapy but OS was not different for patients with low SMI versus high SMI. Moreover, when we compared patients who gained SM and lost SM, we found that patients with a gain of SM had a lower mean SMI at baseline. Many studies on sarcopenia only measure baseline/single time point skeletal muscle which sometimes has an effect on but often does not influence survival $[17 ; 21 ; 30 ; 31]$. In our opinion, measuring SM loss over time is absolutely necessary to adequately identify sarcopenic patients, since important limitations of baseline measurements are that they cannot measure SM loss and are greatly influenced by interpersonal variation of muscle mass and other variables such as obesity and ethnicity. The poor prognostic value of "low baseline SMI" was clearly shown in our data, where low muscle index at baseline could not predict survival and in fact many patients with low baseline SMI gained muscle mass during chemotherapy and were classified as high baseline SMI after neoadjuvant treatment. 
Our results have also shown an association between loss of VAT and a shorter OS. This was also confirmed in other studies [20; 32]. We approach this finding with caution because we have noticed that VAT was not always easily identified and measured in cases where ascites was present before treatment, i.e. in 55\% of patients. Therefore an increase in VAT could represent an increased measurement of VAT due to diminished ascites after chemotherapy treatment rather than an actual increase in VAT due to other causes. Survival gain associated with an increase of VAT could thus reflect the group of patients with a good response to chemotherapy. However this hypothesis cannot be confirmed in our data because patients with ascites were divided equally among the women who gained VAT and the women who lost VAT during chemotherapy.

Other authors in the field have found mixed results regarding the relationship between muscle and adipose tissue changes and survival. Our findings are concordant with the outcome of a study in non-small-cell lung cancer patients; Stene et al found a longer OS for patients who maintained or gained SM in comparison to patients who lost SM and survival was irrespective of the presence of sarcopenia at baseline [21]. In general, a mean decrease in SM is seen during chemotherapy for patients with lung cancer, pancreatic cancer and oesophagogastric cancer, similar to our findings in ovarian cancer [18-21; 32; 33]. However, this decrease in SM did not alter OS rates in oesophagogastric cancer [18; 19]. In pancreatic cancer, a decline in SM and VAT was recorded during neoadjuvant chemotherapy, but only loss of VAT was associated with a shorter OS [20; 32].

Known prognostic factors such as age, BMI, tumor stage or weight loss may attribute to prognosis but we were not able to find a significant relationship for these variables with OS. As reported by Prado et al, obesity is not protective against muscle loss, on the contrary; sarcopenic obesity was associated with poorer functional status and was an independent predictor of survival [31]. Therefore, an evaluation of bodyweight instead of body composition can be misleading when a patient is assessed.

\section{Limitations}

Due to the retrospective nature of this study, it was not always possible to retrieve all variables and possible confounders of interest in all patients. Body weight - and BMI both before the start of treatment as well as during chemotherapy were not retrievable for all subjects. It would also have been interesting to incorporate WHO performance status and tumor marker CA125 into the analyses, but due to too many missing data this was not possible.

A second important shortcoming of this study is selection bias. Instead of studying all patients with ovarian cancer we only selected the patients with advanced disease who were assigned to neoadjuvant chemotherapy and interval debulking. These patients often have large bulky tumors, more advanced locoregional and distal tumor spread and/or a worse performance status which prevents them from undergoing 
primary surgical treatment. For this study we intentionally made the decision to use this particular group because it gave us the opportunity to compare muscle loss over the course of time due to the availability of multiple CT scans. Patients who are treated with primary debulking only receive one CT scan before treatment and are not followed-up with CT during adjuvant chemotherapy unless a suspicion of recurrence arises. Whether our results can be reproduced in ovarian cancer patients who receive primary cytoreductive surgery without neoadjuvant chemotherapy will be a topic of future research.

Furthermore, our study took place in a specialised oncologic centre in the south of the Netherlands. Survival rates could vary between specialised and non-specialised centres but also countries or health care systems across the world. Therefore, we believe it is important that body composition measurement studies for gynaecological malignancies should be validated in other populations.

Unlike many other published manuscripts, we decided to use our own cut-off value to define sarcopenia in our population. Other authors refer to the study of Prado et al in which a value of 38.5 for SMI is used to define sarcopenic patients but this cut-off value is based on obese patients with respiratory and gastrointestinal tumors and we did not find this cut-off representative for the present study population [31]. The use of a different and lower cut-off value (e.g. 38.5) would have resulted in a higher number of sarcopenic patients; we tested whether this significantly changed any of the outcomes but found that this was not the case in both the Kaplan-Meier and Cox-regression analyses (data not shown). Instead, we decided to set our own cut-offs which is a more unbiased approach.

\section{CONCLUSION AND IMPLICATIONS FOR PRACTICE AND RESEARCH}

In this manuscript we have provided evidence that loss of skeletal muscle and loss of visceral adipose tissue during neoadjuvant chemotherapy is detrimental to overall survival for ovarian cancer patients. Evaluation of skeletal muscle at a specific time point does not help in predicting survival which is why we propose a measurement over time to adequately identify sarcopenic patients. External and prospective validation of these findings in other cohorts from (inter)national centres is imperative. However, even more important are prospective randomised controlled trials investigating whether nutritional, pharmacological and/or physical interventions to maintain or even increase skeletal muscle and adipose tissue can improve overall survival in ovarian cancer patients. Nutritional intervention schemes have been developed for ovarian cancer patients and cancer patients in general, largely based on expert opinions and often lacking clinical evidence [34; 35]. 


\section{REFERENCES}

1 Ferlay J, Soerjomataram I, Ervik M et al GLOBOCAN 2012 v1.0, Cancer Incidence and Mortality Worldwide: IARC CancerBase No. 11, 2013.

2 Surveillance E, and End Results (SEER) Program SEER 9 Incidence \& U.S. Mortality Research Data (19732011). In: National Cancer Institute D, Surveillance Research Program, Surveillance Systems Branch, released April 2014, based on the November 2013 submission, (ed),

3 De Angelis R, Sant M, Coleman MP et al (2014) Cancer survival in Europe 1999-2007 by country and age: results of EUROCARE--5-a population-based study. Lancet Oncol 15:23-34

4 du Bois A, Reuss A, Pujade-Lauraine E, Harter P, Ray-Coquard I, Pfisterer J (2009) Role of surgical outcome as prognostic factor in advanced epithelial ovarian cancer: a combined exploratory analysis of 3 prospectively randomized phase 3 multicenter trials: by the Arbeitsgemeinschaft Gynaekologische Onkologie Studiengruppe Ovarialkarzinom (AGO-OVAR) and the Groupe d'Investigateurs Nationaux Pour les Etudes des Cancers de I'Ovaire (GINECO). Cancer 115:1234-1244

5 Vergote I, Trope CG, Amant F et al (2010) Neoadjuvant chemotherapy or primary surgery in stage IIIC or IV ovarian cancer. N Engl J Med 363:943-953

6 Tisdale MJ (2002) Cachexia in cancer patients. Nat Rev Cancer 2:862-871

7 Mourtzakis M, Prado CM, Lieffers JR, Reiman T, McCargar LJ, Baracos VE (2008) A practical and precise approach to quantification of body composition in cancer patients using computed tomography images acquired during routine care. Appl Physiol Nutr Metab 33:997-1006

8 Ross R (2003) Advances in the application of imaging methods in applied and clinical physiology. Acta Diabetol 40 Suppl 1:S45-50

9 Shen W, Punyanitya M, Wang Z et al (2004) Total body skeletal muscle and adipose tissue volumes: estimation from a single abdominal cross-sectional image. J Appl Physiol (1985) 97:2333-2338

10 Shen W, Punyanitya M, Wang Z et al (2004) Visceral adipose tissue: relations between single-slice areas and total volume. Am J Clin Nutr 80:271-278

11 Fujiwara N, Nakagawa H, Kudo Y et al (2015) Sarcopenia, Intramuscular Fat Deposition, and Visceral Adiposity Independently Predict the Outcomes of Hepatocellular Carcinoma. J Hepatol. 10.1016/j.jhep.2015.02.031

12 Lanic H, Kraut-Tauzia J, Modzelewski R et al (2014) Sarcopenia is an independent prognostic factor in elderly patients with diffuse large B-cell lymphoma treated with immunochemotherapy. Leuk Lymphoma 55:817-823

13 Martin L, Birdsell L, Macdonald N et al (2013) Cancer cachexia in the age of obesity: skeletal muscle depletion is a powerful prognostic factor, independent of body mass index. J Clin Oncol 31:1539-1547

14 Psutka SP, Carrasco A, Schmit GD et al (2014) Sarcopenia in patients with bladder cancer undergoing radical cystectomy: impact on cancer-specific and all-cause mortality. Cancer 120:2910-2918

15 Guiu B, Petit JM, Bonnetain F et al (2010) Visceral fat area is an independent predictive biomarker of outcome after first-line bevacizumab-based treatment in metastatic colorectal cancer. Gut 59:341-347

16 Ladoire S, Bonnetain F, Gauthier M et al (2011) Visceral fat area as a new independent predictive factor of survival in patients with metastatic renal cell carcinoma treated with antiangiogenic agents. Oncologist 16:71-81

17 Torres ML, Hartmann LC, Cliby WA et al (2013) Nutritional status, CT body composition measures and survival in ovarian cancer. Gynecol Oncol 129:548-553

18 Awad S, Tan BH, Cui H et al (2012) Marked changes in body composition following neoadjuvant chemotherapy for oesophagogastric cancer. Clin Nutr 31:74-77

19 Yip C, Goh V, Davies A et al (2014) Assessment of sarcopenia and changes in body composition after neoadjuvant chemotherapy and associations with clinical outcomes in oesophageal cancer. Eur Radiol 24:998-1005 


\section{Chapter 2}

20 Dalal S, Hui D, Bidaut L et al (2012) Relationships among body mass index, longitudinal body composition alterations, and survival in patients with locally advanced pancreatic cancer receiving chemoradiation: a pilot study. J Pain Symptom Manage 44:181-191

21 Stene GB, Helbostad JL, Amundsen T et al (2015) Changes in skeletal muscle mass during palliative chemotherapy in patients with advanced lung cancer. Acta Oncol 54:340-348

22 Mugel T, Ghossain M, Buy JN, Malbec L, Vadrot D (1993) [Value of x-ray computed tomography and MRI in primary tumors of the ovary]. J Radiol 74:151-156

23 Fearon K, Arends J, Baracos V (2013) Understanding the mechanisms and treatment options in cancer cachexia. Nat Rev Clin Oncol 10:90-99

24 Kortebein P, Ferrando A, Lombeida J, Wolfe R, Evans WJ (2007) Effect of 10 days of bed rest on skeletal muscle in healthy older adults. JAMA 297:1772-1774

25 Schiesser M, Kirchhoff P, Muller MK, Schafer M, Clavien PA (2009) The correlation of nutrition risk index, nutrition risk score, and bioimpedance analysis with postoperative complications in patients undergoing gastrointestinal surgery. Surgery 145:519-526

26 Alphs HH, Zahurak ML, Bristow RE, Diaz-Montes TP (2006) Predictors of surgical outcome and survival among elderly women diagnosed with ovarian and primary peritoneal cancer. Gynecol Oncol 103:10481053

27 Baracos V, Kazemi-Bajestani SM (2013) Clinical outcomes related to muscle mass in humans with cancer and catabolic illnesses. Int J Biochem Cell Biol 45:2302-2308

28 Prado CM, Lieffers JR, Bowthorpe L, Baracos VE, Mourtzakis M, McCargar LJ (2013) Sarcopenia and physical function in overweight patients with advanced cancer. Can J Diet Pract Res 74:69-74

29 Prado CM, Baracos VE, McCargar LJ et al (2009) Sarcopenia as a determinant of chemotherapy toxicity and time to tumor progression in metastatic breast cancer patients receiving capecitabine treatment. Clin Cancer Res 15:2920-2926

30 Kuroki LM, Mangano M, Allsworth JE et al (2015) Pre-operative Assessment of Muscle Mass to Predict Surgical Complications and Prognosis in Patients With Endometrial Cancer. Ann Surg Oncol 22:972-979

31 Prado CM, Lieffers JR, McCargar LJ et al (2008) Prevalence and clinical implications of sarcopenic obesity in patients with solid tumours of the respiratory and gastrointestinal tracts: a population-based study. Lancet Oncol 9:629-635

32 Cooper AB, Slack R, Fogelman D et al (2014) Characterization of Anthropometric Changes that Occur During Neoadjuvant Therapy for Potentially Resectable Pancreatic Cancer. Ann Surg Oncol. 10.1245/s10434-014-4285-2

33 Murphy RA, Mourtzakis M, Chu QS, Baracos VE, Reiman T, Mazurak VC (2011) Nutritional intervention with fish oil provides a benefit over standard of care for weight and skeletal muscle mass in patients with nonsmall cell lung cancer receiving chemotherapy. Cancer 117:1775-1782

34 Jacquelin-Ravel N, Pichard C (2012) Clinical nutrition, body composition and oncology: a critical literature review of the synergies. Crit Rev Oncol Hematol 84:37-46

35 Balogun N, Forbes A, Widschwendter M, Lanceley A (2012) Noninvasive nutritional management of ovarian cancer patients: beyond intestinal obstruction. Int J Gynecol Cancer 22:1089-1095 



\section{Chapter 3}

\section{ABSTRACT}

Background: Sarcopenia, severe skeletal muscle loss, has been identified as a prognostic factor in various malignancies. This study aims to investigate whether sarcopenia is associated with overall survival (OS) and surgical complications in patients with advanced ovarian cancer undergoing primary debulking surgery (PDS).

Methods: Ovarian cancer patients $(n=216)$ treated with PDS were enrolled retrospectively. Total skeletal muscle surface area was measured on axial computed tomography at the level of the third lumbar vertebra. Optimum stratification was used to find the optimal skeletal muscle index cut-off to define sarcopenia $\left(\leq 38.73 \mathrm{~cm}^{2} / \mathrm{m}^{2}\right)$. Cox-regression and Kaplan-Meier analysis were used to analyse the relationship between sarcopenia and OS. The effect of sarcopenia on the development of major surgical complications was studied with logistic regression.

Results: Kaplan-Meier analysis showed a significant survival disadvantage for patients with sarcopenia compared to patients without sarcopenia $(p=0.010)$. Sarcopenia univariably predicted OS (HR $1.536(95 \% \mathrm{Cl} 1.105-2.134), \mathrm{p}=0.011)$ but was not significant in multivariable Cox-regression analysis (HR $1.362(95 \% \mathrm{Cl} \quad 0.968-1.916)$ $\mathrm{p}=0.076$ ). Significant predictors for OS in multivariable Cox-regression analysis were complete PDS, treatment in a specialised centre and the development of major complications. Sarcopenia was not predictive of major complications.

Conclusion: Sarcopenia was not predictive of OS or major complications in ovarian cancer patients undergoing primary debulking surgery. However a strong trend towards a survival disadvantage for patients with sarcopenia was seen. Future prospective studies should focus on interventions to prevent or reverse sarcopenia and possibly increase ovarian cancer survival. Complete cytoreduction remains the strongest predictor of ovarian cancer survival. 


\section{INTRODUCTION}

Ovarian cancer is the fifth leading cause of cancer-related death among women in developed countries and claims over 150.000 lives worldwide yearly [1]. The majority of patients have abdominally metastasized disease at first presentation which resembles a 5-year survival of 17-36\% [2]. Preferred treatment for patients with advanced FIGO stage (International Federation of Gynecology and Obstetrics) ovarian cancer is upfront primary debulking surgery (PDS). For patients who are considered inoperable or have estimated irresectable disease, neoadjuvant chemotherapy complemented with interval debulking surgery (IDS) provides a legitimate alternative [3]. To remove all visible tumour at surgery is pivotal as the amount of residual disease after surgery is the most influential prognostic factor contributing to ovarian cancer survival along with FIGO stage, tumour grade and performance status [4]. Additionally women with ovarian cancer often experience cancer cachexia characterised by involuntary weight loss and severe muscle wasting which can further abate their survival chances [5]. The mechanisms behind cancer cachexia and muscle depletion involve an interplay between reduced nutrient intake and abnormal metabolism incited by an excessive systemic inflammation and catabolic (tumour-related) mediators to which the host (i.e. the patient) is unable to respond properly [6]. However, cancer-related weight loss can be disguised by bulky tumour deposits or ascites and is less reliable as a measure of disease activity in ovarian cancer patients. Instead of weight measurements, measurements of body composition are now extensively being used for prognostic purposes in cancer patients. Cross-sectional computed tomography (CT) scans at the level of the third lumbar vertebra (L3) give an accurate representation of total body adipose and skeletal muscle tissue [7-9]. Severe loss of muscle mass (i.e. sarcopenia) as estimated on CT was henceforth identified as a risk factor for survival in patients with various malignancies [10-13]. In a cohort of ovarian cancer patients undergoing neoadjuvant chemotherapy and IDS, loss of skeletal muscle over time was also related to decreased survival [14]. However two earlier studies could not find a relationship between sarcopenia and survival when evaluating ovarian cancer patients treated with PDS [15; 16]. The association between sarcopenia and postoperative complications is thus far unknown in ovarian cancer but has shown significance in gastro-intestinal cancer [17; 18].

The data on ovarian cancer patients are scarce but there is evidence that sarcopenia could be one of the few modifiable risk factors for survival if patients with sarcopenia were to be identified timely. The reversal of sarcopenia with nutritional supplements and physical activity has been studied successfully in sarcopenic elderly [19]. Exercise might play an important role in counteracting muscle wasting through reducing the level of inflammation, increasing insulin sensitivity and modulation of muscle protein metabolism [20; 21]. Although there is no direct evidence that anti-sarcopenia treatment increases survival chances in patients with advanced malignancies there is a rationale for implementing e.g. exercise therapy in oncologic patients with sarcopenia 


\section{Chapter 3}

[22]. The primary objective of this study is to evaluate whether sarcopenia is associated with survival and the development of major postoperative complications in patients with advanced ovarian cancer undergoing PDS.

\section{PATIENTS AND METHODS}

This study has been performed in accordance with the ethical standards laid down in the 1964 Declaration of Helsinki. The study protocol has been approved by the Maastricht University Medical Centre ethics committee which waived the requirement to obtain informed consent.

\section{Eligible patients}

All patients with advanced stage ovarian cancer (FIGO IIB-IV) treated with PDS between 2000 and 2015 were included in this retrospective cohort study. Patients underwent treatment in one of two specialised oncologic centres (Maastricht University or Radboud University medical centre) or in one of four general care centres in the Netherlands (Bernhoven, Rijnstate, Slingeland or St. Jansdal). The following inclusion criteria were applied: 1) an abdominal CT-scan of sufficient quality taken within two months before PDS was available, 2) relevant clinical data were retrievable from the patient's medical records and 3) follow-up data were available for at least six months post-surgery.

Primary outcome was overall survival (OS), calculated as the time between surgery and death of any cause. Survivors were censored at a fixed date no sooner than six months after inclusion of the last patient. Postoperative complications were graded using the Clavien-Dindo scale of surgical complications [23]. We classified a ClavienDindo score of $\geq 3$ as a major complication. The result of PDS was categorised into complete (no macroscopic tumour residual), optimal (largest tumour residual $\leq 1 \mathrm{~cm}$ ) or incomplete (largest tumour residual $>1 \mathrm{~cm}$ ). Patients were divided into two age groups with a threshold of 65 years. A body mass index (BMI) $>30$ was considered obese.

\section{BODY COMPOSITION ANALYSIS}

Axial CT at L3 was used for evaluation of total skeletal muscle (SM), psoas muscle, intramuscular adipose tissue (IMAT), visceral adipose tissue (VAT) and subcutaneous adipose tissue (SAT). Predefined and previously validated boundaries of -30 to +150 Hounsfield Units (HU) for SM and psoas, -190 to -30 HU for IMAT and SAT, and -150 to $50 \mathrm{HU}$ for VAT were used to demarcate tissue using SliceOmatic software (v5.0, Tomovision, Montreal, Canada), see figure 1. Surface areas in $\mathrm{cm}^{2}$ were quantified 
automatically following demarcation and were standardised by height squared to compute the skeletal muscle index (SMI), psoas-index, IMAT-index, VAT-index and SATindex in $\mathrm{cm}^{2} / \mathrm{m}^{2}$. Mean $\mathrm{HU}$ was calculated for SM to define the muscle radiation attenuation (MA). CT evaluation was performed by two observers (IR\&JU) blinded to each other and to patient outcome. Their averaged measurements were used for statistical analysis.

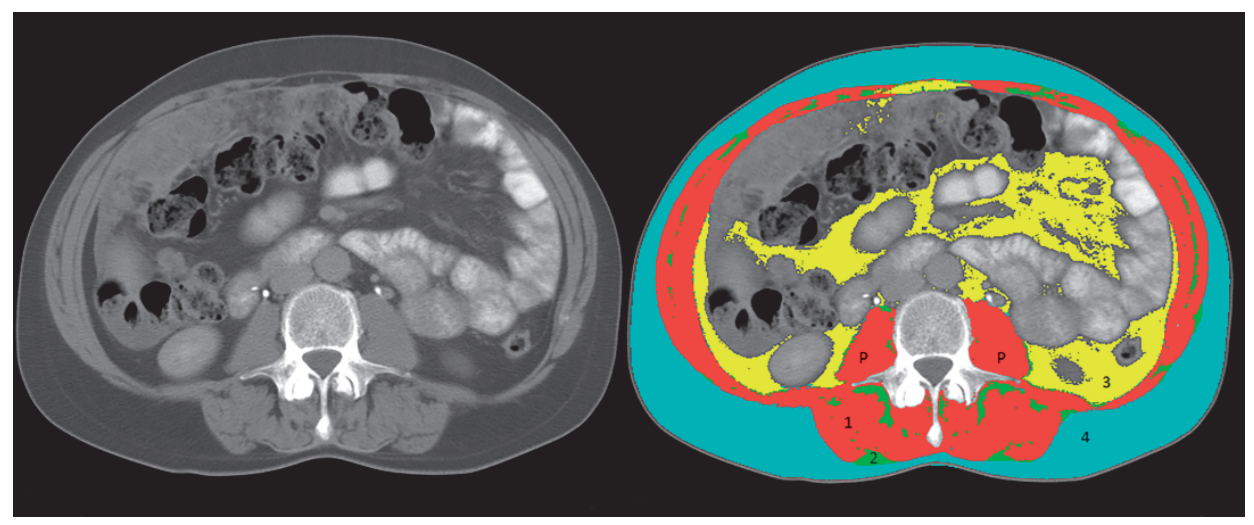

Figure 1: Computed tomography assessment of skeletal muscle and adipose tissue with SliceOmatic software Preoperative axial computed tomography (CT) scan of an ovarian cancer patient. Both images are taken from the same patient. On the left a regular CT image at the level of the third lumbar vertebra. On the right an image coloured using SliceOmatic software. 1= skeletal muscle, 2 = intramuscular adipose tissue, $3=$ visceral adipose tissue, 4 = subcutaneous adipose tissue, $\mathrm{P}=$ psoas muscle.

\section{STATISTICAL ANALYSIS}

We performed optimum stratification analysis on the SMI measurements to stratify patients into two groups: patients with sarcopenia and patients without sarcopenia. This method produces a p-value for each SMI cut-off and determines the optimal cut-off for sarcopenia in our population [24]. Psoas-index, IMAT-index, VAT-index, SAT-index and MA were divided into tertiles and the lowest tertile was used as cut-off for each category (with an exception for IMAT-index in which the highest tertile was used) to divide patients into a 'low' or 'high' subgroup. Baseline data between patients with and without sarcopenia were analysed with chi-squared and t-tests. Differences in OS between sarcopenic and non-sarcopenic patients were tested with the Kaplan-Meier estimate (log rank). Body composition measurements as described above, FIGO stage (<IV vs. IV), age, BMI, outcome of PDS (complete vs. optimal/incomplete), tumour grade (1 vs. 2/3), treatment centre (specialised vs. general), type of treatment (PDS vs. PDS+IDS) and development of major complications (Clavien-Dindo $\geq 3$ ) were tested as effect modifiers in regression analysis adopting a backwards stepwise procedure. A proportional hazards 


\section{Chapter 3}

Cox-regression model was used to evaluate relationships with OS and a binary logistic regression model was used to study predictors for the development of major complications at PDS. Hazard ratios (HR) were calculated with confidence intervals $(95 \% \mathrm{Cl})$. For univariable analysis an inclusion criterion of $10 \%$ was adopted. Significant modifiers were included in a multivariable model in a backwards stepwise procedure in which a p-value $<0.05$ was considered significant. For all statistical analyses SPSS v23.0 (IBM Corp, Chicago, IL) was used. Interobserver agreement for measurement of body composition variables was calculated with Pearson's correlation coefficient $\left(r_{p}\right)$. Pearson's $r$ was also used to evaluate the correlation between SMI and psoas-index.

\section{RESULTS}

Of 280 patients deemed eligible to participate, 64 were excluded. 216 patients were included in the analyses (Figure 2). Patient characteristics are summarised in Table 1. Mean period between CT and PDS was 21 days. Mean OS was 1714 days. Forty-three patients (19.9\%) experienced a major postoperative complication. A more elaborate list of complications can be found in the supplementary material. Sarcopenic patients had a significantly lower mean BMI compared to non-sarcopenic patients $\left(22.7 \mathrm{vs} .26 .0 \mathrm{~kg} / \mathrm{m}^{2}\right.$, $\mathrm{p}<0.001)$. Ascites was more prevalent in sarcopenic patients $(87.1 \%$ versus $65.7 \%$, $\mathrm{p}=0.010)$. Furthermore SMI, psoas-index, VAT-index and SAT-index were all significantly lower in patients with sarcopenia (all $p<0.001$ ). The outcome of PDS was not significantly different between sarcopenic and non-sarcopenic patients $(25.7 \%$ vs. $35.6 \%$ complete, $p=0.346$ ).

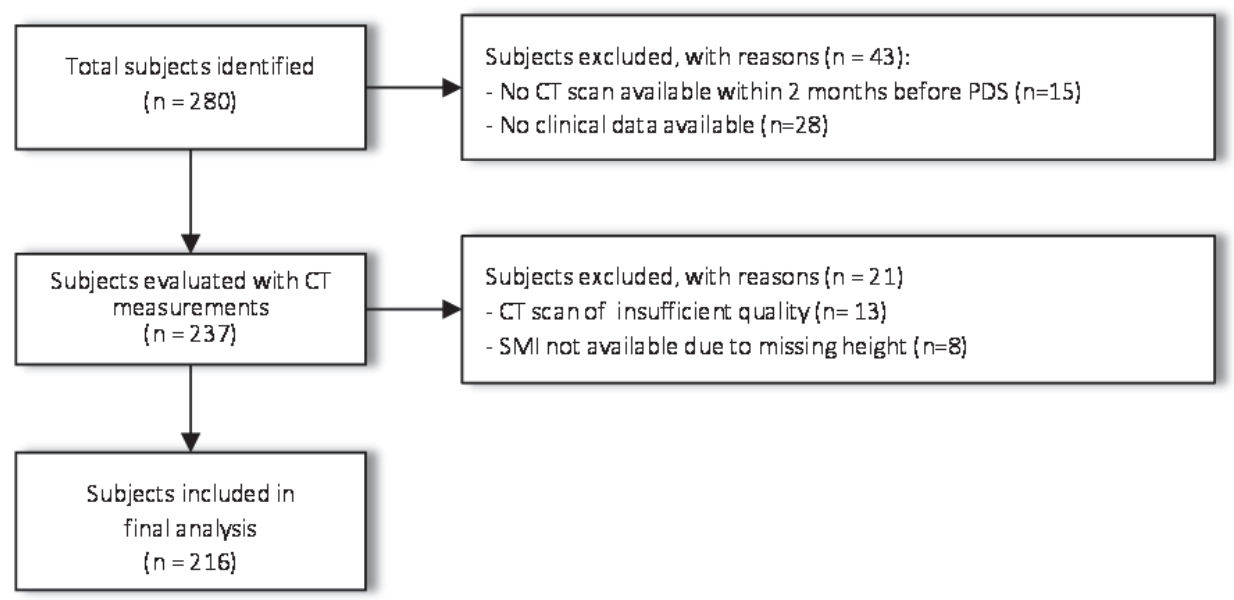

Figure 2: Flow chart for patient inclusion $\mathrm{CT}$; computed tomography, PDS; primary debulking surgery, SMI; skeletal muscle index 
Table 1: Patient characteristics

\begin{tabular}{|c|c|c|c|}
\hline & $\begin{array}{l}\text { All patients } \\
(n=216)\end{array}$ & $\begin{array}{l}\text { Sarcopenia } \\
(n=70)\end{array}$ & $\begin{array}{l}\text { No Sarcopenia } \\
(n=146)\end{array}$ \\
\hline \multicolumn{4}{|l|}{ Patient and tumour characteristics } \\
\hline Age in years, mean \pm SE (range) & $63.1 \pm 0.8(16-85)$ & $64.2 \pm 1.4(27-85)$ & $62.5 \pm 1.1(16-85)$ \\
\hline Body Mass Index in $\mathrm{kg} / \mathrm{m}^{2}$, mean $\pm \mathrm{SE}$ & $24.9 \pm 0.3$ & $22.7 \pm 0.4^{\mathrm{a}}$ & $26.0 \pm 0.3^{\mathrm{a}}$ \\
\hline \multicolumn{4}{|l|}{ FIGO tumour stage, n (\%) } \\
\hline II & $12(5.6)$ & $1(1.4)$ & $11(7.5)$ \\
\hline III & $161(74.5)$ & $50(71.4)$ & $111(76.0)$ \\
\hline IV & $43(19.9)$ & $19(27.1)$ & $24(16.4)$ \\
\hline \multicolumn{4}{|l|}{ Tumour grade, $\mathrm{n}(\%)$} \\
\hline 1 & $16(7.4)$ & $4(5.7)$ & $12(8.2)$ \\
\hline 2 & $47(21.8)$ & $12(17.1)$ & $35(24.0)$ \\
\hline 3 & $133(61.6)$ & $50(71.4)$ & $83(56.8)$ \\
\hline Unknown & $20(9.3)$ & $4(5.7)$ & $16(11.0)$ \\
\hline Presence of ascites, n (\%) & $157(72.7)$ & $61(87.1)^{a}$ & $96(65.8)^{a}$ \\
\hline \multicolumn{4}{|l|}{ Measurements } \\
\hline $\mathrm{SMI}$ in $\mathrm{cm}^{2} / \mathrm{m}^{2}$, mean $\pm \mathrm{SE}$ (cut-point) & $41.99 \pm 0.39(38.73)$ & $35.73 \pm 0.28^{a}$ & $44.98 \pm 0.34^{a}$ \\
\hline Psoas-index in $\mathrm{cm}^{2} / \mathrm{m}^{2}$, mean $\pm \mathrm{SE}$ (cut-point) & $5.34 \pm 0.08(4.65)$ & $4.64 \pm 0.10^{a}$ & $5.68 \pm 0.10^{a}$ \\
\hline IMAT-index in $\mathrm{cm}^{2} / \mathrm{m}^{2}$, mean $\pm \mathrm{SE}$ (cut-point) & $5.28 \pm 0.22(3.51)$ & $4.75 \pm 0.34$ & $5.54 \pm 0.28$ \\
\hline VAT-index in $\mathrm{cm}^{2} / \mathrm{m}^{2}$, mean $\pm \mathrm{SE}$ (cut-point) & $27.11 \pm 1.45(13.22)$ & $19.68 \pm 1.87^{\mathrm{a}}$ & $30.66 \pm 1.88^{a}$ \\
\hline SAT-index in $\mathrm{cm}^{2} / \mathrm{m}^{2}$, mean $\pm \mathrm{SE}$ (cut-point) & $62.40 \pm 2.29(44.52)$ & $47.65 \pm 2.84^{\mathrm{a}}$ & $69.47 \pm 2.94^{\mathrm{a}}$ \\
\hline MA in $\mathrm{HU}$, mean \pm SE (cut-point) & $36.64 \pm 0.62(33.67)$ & $37.06 \pm 1.14$ & $36.43 \pm 0.73$ \\
\hline \multicolumn{4}{|l|}{ Surgical outcome } \\
\hline \multicolumn{4}{|l|}{ Outcome PDS, n (\%) } \\
\hline Complete & $70(32.4)$ & $18(25.7)$ & $52(35.6)$ \\
\hline Optimal & $51(23.6)$ & $18(25.7)$ & $33(22.6)$ \\
\hline Incomplete & $95(44.0)$ & $34(48.6)$ & $61(41.8)$ \\
\hline Blood loss in $\mathrm{mL}$, mean $\pm \mathrm{SE}$ & $1438 \pm 119$ & $1614 \pm 231$ & $1350 \pm 137$ \\
\hline Length of hospital stay in days, mean $\pm \mathrm{SE}$ & $14.2 \pm 0.8$ & $15.3 \pm 1.6$ & $13.6 \pm 0.9$ \\
\hline Re-admitted within 30 days, $\mathrm{n}(\%)$ & $13(6.0)$ & $5(7.5)$ & $8(5.7)$ \\
\hline \multicolumn{4}{|l|}{ Clavien-Dindo complications scale, n (\%) } \\
\hline Grade O (No complications) & $121(56.0)$ & $36(51.4)$ & $85(58.2)$ \\
\hline $\begin{array}{l}\text { Grade I (Any deviation from normal } \\
\text { postoperative course) }\end{array}$ & $14(6.5)$ & $4(5.7)$ & $10(6.8)$ \\
\hline $\begin{array}{l}\text { Grade II (Requiring pharmacological } \\
\text { treatment) }\end{array}$ & $33(15.3)$ & $14(20.0)$ & $19(13.0)$ \\
\hline Grade III (Requiring invasive intervention) & $22(10.2)$ & $7(10.0)$ & $15(10.3)$ \\
\hline Grade IV (Life-threatening requiring ICU) & $15(6.9)$ & $6(8.6)$ & $9(6.2)$ \\
\hline Grade V (Death) & $6(2.8)$ & $2(2.9)$ & $4(2.7)$ \\
\hline Unknown & $5(2.3)$ & $1(1.4)$ & $4(2.7)$ \\
\hline \multicolumn{4}{|l|}{ Treatment, n (\%) } \\
\hline PDS & $154(71.3)$ & $47(67.1)$ & $107(73.3)$ \\
\hline PDS + secondary IDS after chemotherapy & $62(28.7)$ & $23(32.9)$ & $39(26.7)$ \\
\hline \multicolumn{4}{|l|}{ Treatment centre, n (\%) } \\
\hline General care centre & $99(45.8)$ & $33(47.1)$ & $66(45.2)$ \\
\hline Specialised oncologic centre & $117(54.2)$ & 37 (52.9) & $80(54.8)$ \\
\hline 30-day mortality, n (\%) & $7(3.2)$ & $2(2.9)$ & $5(3.4)$ \\
\hline OS in days, mean \pm SE & $1714 \pm 117$ & $1309 \pm 162^{\mathrm{a}}$ & $1887 \pm 147^{\mathrm{a}}$ \\
\hline
\end{tabular}




\section{Chapter 3}

SE; standard error, FIGO; International Federation of Gynecology and Obstetrics, SMI; skeletal muscle index, IMAT; intramuscular adipose tissue, VAT; visceral adipose tissue, SAT; subcutaneous adipose tissue, MA; muscle radiation attenuation, $\mathrm{HU}$; Hounsfield Units, PDS; primary debulking surgery, IDS; interval debulking surgery, ICU; intensive care unit, OS; overall survival. "Indicates significant difference between "Sarcopenia" and "No sarcopenia" ( $P<0.05$, independent-samples T-test, chi-squared test or log rank test).

Optimum stratification analysis identified an $\mathrm{SMI} \leq 38.73 \mathrm{~cm}^{2} / \mathrm{m}^{2}$ as the most optimal cut-point for sarcopenia in our population. Using this cut-point, Kaplan-Meier analysis showed a significant survival disadvantage for patients with sarcopenia compared to patients without sarcopenia ( $p=0.010$, Figure 3 ). Sarcopenia univariably predicted OS ( HR $1.536(95 \% \mathrm{Cl} 1.105-2.134), p=0.011)$ but was not significant at the level of $5 \%$ in multivariable Cox-regression analysis (HR 1.362 (95\% Cl 0.968-1.916) p=0.076, Table 2). Significant predictors for OS in multivariable Cox-regression analysis were complete PDS (HR 0.545 (95\% Cl 0.366-0.812), p=0.003), specialised centre (HR $0.710(95 \% \mathrm{Cl} 0.506-$ $0.995) \mathrm{p}=0.047)$ and the development of major complications (HR $1.670(95 \% \mathrm{Cl} 1.125$ 2.480), $p=0.011$ ).

Sarcopenic patients did not develop more severe complications than non-sarcopenic patients (Table 1). Sarcopenia was also not predictive of a major complication in logistic regression analysis (Table 2). Age and low MA were univariably identified as predictors for major complications but were not significant in multivariable analysis (Table 2).

Interobserver agreement was 0.970, 0.989, 0.969, 0.997, 0.994 and 0.995 for SM, psoas, IMAT, VAT, SAT and MA respectively. Correlation between measurements of SMI and psoas-index was 0.453 .

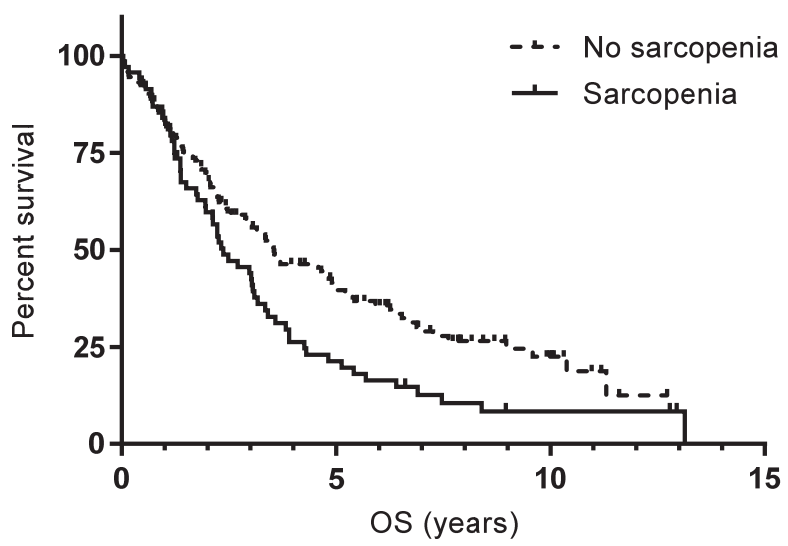

Number of subjects at risk (\%)

\begin{tabular}{llllll} 
& $0 \mathrm{yr}$ & $1 \mathrm{yr}$ & $2 \mathrm{yr}$ & $5 \mathrm{yr}$ & $10 \mathrm{yr}$ \\
\hline No Sarcopenia & $146(100)$ & $117(80)$ & $90(62)$ & $44(30)$ & $10(7)$ \\
Sarcopenia & $70(100)$ & $57(81)$ & $40(57)$ & $14(20)$ & $4(6)$ \\
\hline
\end{tabular}

Figure 3: Kaplan-Meier survival analysis

OS; overall survival. Log rank estimate: $p=0.010$. 


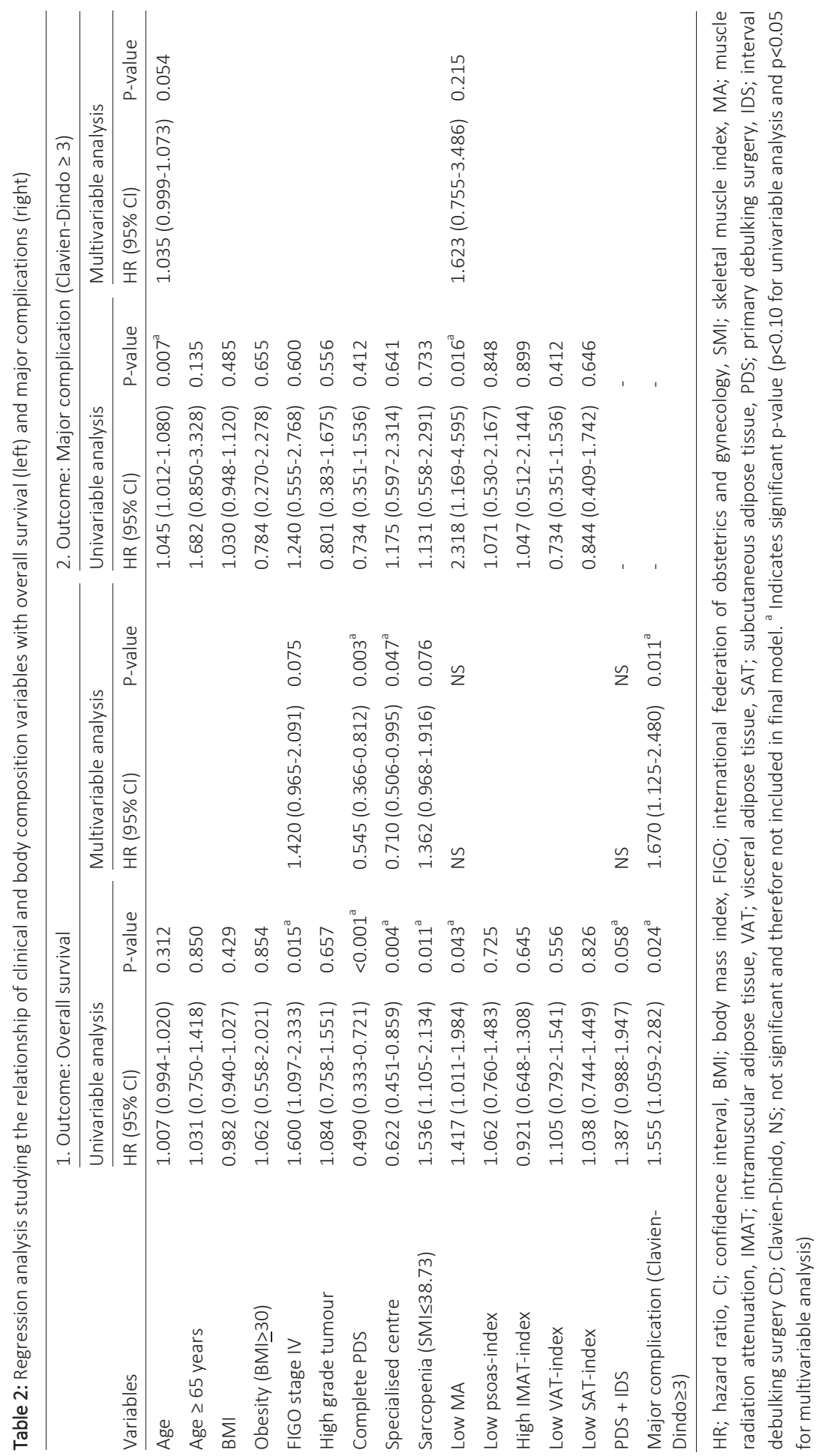




\section{DISCUSSION}

In this study we evaluated the role of skeletal muscle measurements in prediction of OS and the development of post-surgical complications in ovarian cancer patients treated with PDS. We found a strong trend towards a survival disadvantage for patients with sarcopenia but the relationship was not significant in multivariable regression analysis. Tumour stage, completeness of PDS, treatment in a specialised centre and the development of severe post-surgical complications were stronger predictors for ovarian cancer survival. Secondly we did not establish a relationship between sarcopenia and the development of complications after PDS.

A recent meta-analysis concluded that low SMI was associated with poor OS among various tumour types [13]. Unfortunately no gynaecological malignancies were included in the meta-analysis and individual studies on ovarian cancer have not been able to confirm this association. Two retrospective studies about ovarian cancer patients undergoing PDS found no relationship between low SM and survival [15; 16]. Further, in a cohort of ovarian cancer patients undergoing neoadjuvant chemotherapy and IDS an initial low SMI measurement before treatment had no prognostic relevance but a change in muscle during chemotherapy was highly predictive of OS [14]. The present study shows no significant relationship between sarcopenia and OS when corrected for other prognostic variables but does imply a tendency towards a shorter survival nonetheless as is seen in Kaplan-Meier analysis and univariable regression. It is possible that the effect of sarcopenia was substantially diminished due to the influence of other strong prognostic predictors such as the outcome of debulking surgery. Furthermore, the impact of sarcopenia on OS might become more apparent when studying a larger population, which may also explain why previously published (relatively small) studies were not able to show a connection between sarcopenia and survival.

We encountered some obstacles in this study when we were faced with the choice of a cut-point for sarcopenia. SMI cut-points of $29.6-42.1 \mathrm{~cm}^{2} / \mathrm{m}^{2}$ have been reported for female cancer patients [13]. Although the majority of studies have used a cut-point between $38.5-41.0 \mathrm{~cm}^{2} / \mathrm{m}^{2}$ for females, this has still resulted in a very heterogeneous reported incidence of $15-74 \%$ patients classifying as sarcopenic [13]. Cancer type and stage, interpersonal variation of muscle mass, obesity and ethnicity can all influence SMI and to define a single 'gold standard' cut-point for sarcopenia is virtually impossible $[25 ; 26]$. We revised previous oncologic studies but cut-points for gynaecological cancer patients specifically were non-existent. In our opinion cut-points designed for and applied to gastrointestinal cancer patients were less applicable to our population since these cancers have a stronger relationship with nutrition and metabolism in general. Although gynaecological tumours and urological tumours have differences in presentation, pathology and prognosis, they both do not affect nutrient uptake and metabolism directly Due to a lack of other suitable comparable cancers we investigated cut-points used in populations with urological cancers. Psutka et al. studied patients 
with renal cell cancer and urothelial cancer and found a relationship between sarcopenia and survival when using an SMI cut-point of $39 \mathrm{~cm}^{2} / \mathrm{m}^{2}$ [11; 27]. The cutpoint of $39 \mathrm{~cm}^{2} / \mathrm{m}^{2}$ in these studies was chosen in accordance with guidelines defined by an international consensus group [6]. However, this guideline dates back to 2011 and was strongly based on the outcome of a study investigating respiratory and gastrointestinal tumours in an obese population [28]. Due to the absence of a suitable existing cut-point for ovarian cancer patients we used optimum stratification to determine the optimal cut-point for sarcopenia in our own cohort. To investigate if a different sarcopenia cut-point would influence the relationship with OS we performed a post-hoc analysis using two alternative cut-points: 1) $39 \mathrm{~cm}^{2} / \mathrm{m}^{2}$ from the existing literature on urological tumours and 2) the lowest tertile SMI from our own population which was $38.87 \mathrm{~cm}^{2} / \mathrm{m}^{2}$. These cut-points were very close to the cut-point of 38.73 $\mathrm{cm}^{2} / \mathrm{m}^{2}$ used in this study and expectedly did not have a substantial impact on outcome. Both alternative cut-points resulted in a significant univariable but not multivariable relationship between sarcopenia and OS although they also showed a strong tendency towards a relation. In fact, all cut-points between 37.90 and 40.25 showed univariable significant relationships with OS for our population.

Interobserver agreement between the two observers was very strong $\left(r_{p}\right.$ 0.9690.997). Quantification of skeletal muscle and adipose tissue with SliceOmatic software is highly reproducible. Although the mean VAT- and SAT-index were significantly lower in patients with sarcopenia compared to patients without sarcopenia, neither measurements of adipose tissue were predictive of OS or complications. The psoasindex weakly correlated with the SMI and was not predictive of survival or complications thus we strongly advise against using it as substitute for the SMI. Aust et al. previously identified MA as a prognostic factor for OS in ovarian cancer patients [16]. We could not establish this relationship in our population.

From our results we can conclude that sarcopenia does not predict the development of major complications after PDS for ovarian cancer. This is in agreement with the only other ovarian cancer study that evaluated sarcopenia in relation to surgical complications [15]. However researchers in other cancer types have been able to connect sarcopenia to the risk of surgical complications although the results are inconsistent. We hypothesize that this difference may be (partially) explained by the aggressiveness of the surgical procedure. The extensiveness of surgery is possibly more limited in ovarian cancer patients than for instance in patients undergoing resections for gastro-intestinal malignancies. When it is apparent that a patient with ovarian cancer will have to undergo extensive surgery including splenectomy, (partial) hepatectomy, and/or multiple resections of large or small bowel, patients are often primarily treated with neoadjuvant chemotherapy [3; 29]. In this way the risk of complications during a possible interval debulking is decreased with an expected decrease of tumour burden. Patients undergoing more extensive surgery are also more likely to develop severe complications which could explain why a relationship between 


\section{Chapter 3}

sarcopenia and complications might be more prominent in other cancer types because the complication rate is higher.

Due to the retrospective nature of our study we encountered a substantial amount of irretrievable data which explains why 64 patients from the original cohort were excluded. We would also have liked to analyse the influence of performance status on sarcopenia and survival but this information was unfortunately missing in over $50 \%$ of patients.

\section{CONCLUSION}

Sarcopenia was not predictive of overall survival or major surgical complications in ovarian cancer patients undergoing primary debulking surgery. Other prognostic factors were stronger predictors for survival. However we did see a strong trend towards a survival disadvantage for patients with sarcopenia. Previous investigations have shown that loss of skeletal muscle during chemotherapy was related to decreased survival in ovarian cancer and the measurement of muscle change might be more important than a single measurement before treatment [14]. Sarcopenia seems to play a noticeable role in ovarian cancer. Whether sarcopenia can be modified with therapy is still unknown. Nutritional and exercise interventions have been found to improve muscle function in sarcopenic elderly [19]. However, readily implementable anti-sarcopenia protocols for cancer patients are unfortunately not yet available. Future prospective studies should focus on investigation of the metabolic phenotype of patients with sarcopenia and to assessing whether interventions (e.g. nutritional support, antiinflammatory medication and physical exercise) have an effect in cancer patients. For the time being it remains pivotal however to achieve complete cytoreduction during ovarian cancer debulking surgery as this has shown to be the strongest predictor of overall survival. 


\section{REFERENCES}

1 Torre LA, Bray F, Siegel RL, Ferlay J, Lortet-Tieulent J, Jemal A (2015) Global cancer statistics, 2012. CA Cancer J Clin 65:87-108

2 Baldwin LA, Huang B, Miller RW et al (2012) Ten-year relative survival for epithelial ovarian cancer. Obstet Gynecol 120:612-618

3 Vergote I, Trope CG, Amant F et al (2010) Neoadjuvant chemotherapy or primary surgery in stage IIIC or IV ovarian cancer. N Engl J Med 363:943-953

4 du Bois A, Reuss A, Pujade-Lauraine E, Harter P, Ray-Coquard I, Pfisterer J (2009) Role of surgical outcome as prognostic factor in advanced epithelial ovarian cancer: a combined exploratory analysis of 3 prospectively randomized phase 3 multicenter trials: by the Arbeitsgemeinschaft Gynaekologische Onkologie Studiengruppe Ovarialkarzinom (AGO-OVAR) and the Groupe d'Investigateurs Nationaux Pour les Etudes des Cancers de I'Ovaire (GINECO). Cancer 115:1234-1244

5 Tisdale MJ (2002) Cachexia in cancer patients. Nat Rev Cancer 2:862-871

6 Fearon K, Strasser F, Anker SD et al (2011) Definition and classification of cancer cachexia: an international consensus. Lancet Oncol 12:489-495

7 Shen W, Punyanitya M, Wang Z et al (2004) Visceral adipose tissue: relations between single-slice areas and total volume. Am J Clin Nutr 80:271-278

8 Shen W, Punyanitya M, Wang $Z$ et al (2004) Total body skeletal muscle and adipose tissue volumes: estimation from a single abdominal cross-sectional image. J Appl Physiol (1985) 97:2333-2338

9 Mourtzakis M, Prado CM, Lieffers JR, Reiman T, McCargar LJ, Baracos VE (2008) A practical and precise approach to quantification of body composition in cancer patients using computed tomography images acquired during routine care. Appl Physiol Nutr Metab 33:997-1006

10 van Vledder MG, Levolger S, Ayez N, Verhoef C, Tran TC, ljzermans JN (2012) Body composition and outcome in patients undergoing resection of colorectal liver metastases. Br J Surg 99:550-557

11 Psutka SP, Boorjian SA, Moynagh MR et al (2016) Decreased Skeletal Muscle Mass is Associated with an Increased Risk of Mortality after Radical Nephrectomy for Localized Renal Cell Cancer. J Urol 195:270276

12 Martin L, Birdsell L, Macdonald N et al (2013) Cancer cachexia in the age of obesity: skeletal muscle depletion is a powerful prognostic factor, independent of body mass index. J Clin Oncol 31:1539-1547

13 Shachar SS, Williams GR, Muss HB, Nishijima TF (2016) Prognostic value of sarcopenia in adults with solid tumours: A meta-analysis and systematic review. Eur J Cancer 57:58-67

14 Rutten IJG, Van Dijk DPJ, Kruitwagen RFPM, Beets-Tan RGH, Olde Damink SW, Van Gorp T (2016) Loss of skeletal muscle during neoadjuvant chemotherapy is related to decreased survival in ovarian cancer patients. Journal of Cachexia, Sarcopenia and Muscle. 10.1002/jcsm.12107

15 Torres ML, Hartmann LC, Cliby WA et al (2013) Nutritional status, CT body composition measures and survival in ovarian cancer. Gynecol Oncol 129:548-553

16 Aust S, Knogler T, Pils D et al (2015) Skeletal Muscle Depletion and Markers for Cancer Cachexia Are Strong Prognostic Factors in Epithelial Ovarian Cancer. PLoS One 10:e0140403

17 Lieffers JR, Bathe OF, Fassbender K, Winget M, Baracos VE (2012) Sarcopenia is associated with postoperative infection and delayed recovery from colorectal cancer resection surgery. Br J Cancer 107:931-936

18 Fukuda Y, Yamamoto K, Hirao M et al (2015) Sarcopenia is associated with severe postoperative complications in elderly gastric cancer patients undergoing gastrectomy. Gastric Cancer. 10.1007/ s10120-015-0546-4

19 Rondanelli M, Klersy C, Terracol G et al (2016) Whey protein, amino acids, and vitamin D supplementation with physical activity increases fat-free mass and strength, functionality, and quality of life and decreases inflammation in sarcopenic elderly. Am J Clin Nutr 103:830-840

20 Gould DW, Lahart I, Carmichael AR, Koutedakis Y, Metsios GS (2013) Cancer cachexia prevention via physical exercise: molecular mechanisms. J Cachexia Sarcopenia Muscle 4:111-124 


\section{Chapter 3}

21 Maddocks M, Murton AJ, Wilcock A (2012) Therapeutic exercise in cancer cachexia. Crit Rev Oncog 17:285-292

22 Bowen TS, Schuler G, Adams V (2015) Skeletal muscle wasting in cachexia and sarcopenia: molecular pathophysiology and impact of exercise training. J Cachexia Sarcopenia Muscle 6:197-207

23 Dindo D, Demartines N, Clavien PA (2004) Classification of surgical complications: a new proposal with evaluation in a cohort of 6336 patients and results of a survey. Ann Surg 240:205-213

24 Williams BA, N. Mandrekar JN, Mandrekar SJ, Cha SS, Furth AF (2006) Finding Optimal Cutpoints for Continuous Covariates with Binary and Time-to-Event Outcomes. Technical Report Series \#79. Mayo Foundation

25 Jones A, Jr., Shen W, St-Onge MP et al (2004) Body-composition differences between African American and white women: relation to resting energy requirements. Am J Clin Nutr 79:780-786

26 Tan L, Liu SL, Lei SF, Papasian CJ, Deng HW (2012) Molecular genetic studies of gene identification for sarcopenia. Hum Genet 131:1-31

27 Psutka SP, Carrasco A, Schmit GD et al (2014) Sarcopenia in patients with bladder cancer undergoing radical cystectomy: impact on cancer-specific and all-cause mortality. Cancer 120:2910-2918

28 Prado CM, Lieffers JR, McCargar $\amalg$ et al (2008) Prevalence and clinical implications of sarcopenic obesity in patients with solid tumours of the respiratory and gastrointestinal tracts: a population-based study. Lancet Oncol 9:629-635

29 Vergote I, du Bois A, Amant F, Heitz F, Leunen K, Harter P (2013) Neoadjuvant chemotherapy in advanced ovarian cancer: On what do we agree and disagree? Gynecol Oncol 128:6-11 


\section{Chapter 4}

\section{ABSTRACT}

Background: Computed tomography measurements of total skeletal muscle area can detect changes and predict overall survival (OS) in patients with advanced ovarian cancer. This study investigates whether assessment of psoas muscle area reflects total muscle area and can be used to assess sarcopenia in ovarian cancer patients.

Methods: Ovarian cancer patients $(n=150)$ treated with induction chemotherapy and interval debulking were enrolled retrospectively in this longitudinal study. Muscle was measured cross-sectionally with computed tomography in three ways: (1) software quantification of total skeletal muscle area (SMA), (2) software quantification of psoas muscle area (PA), and (3) manual measurement of length and width of the psoas muscle to derive the psoas surface area (PLW). Pearson correlation between the different methods was studied. Patients were divided into two groups based on the extent of change in muscle area and agreement was measured with kappa coefficients. Coxregression was used to test predictors for OS.

Results: Correlation between SMA and both psoas muscle area measurements was poor ( $r=0.52$ and 0.39 for PA and PLW, respectively). After categorising patients into muscle loss or gain, kappa agreement was also poor for all comparisons (all $k<0.40$ ). In regression analysis, SMA loss was predictive of poor OS (hazard ratio $1.698(95 \% \mathrm{Cl}$ 1.038-2.778), $P=0.035)$. No relationship with OS was seen for PA or PLW loss.

Conclusions: Change in psoas muscle area is not representative of total muscle area change and should not be used to substitute total skeletal muscle to predict survival in patients with ovarian cancer. 


\section{INTRODUCTION}

Sixty percent of patients diagnosed with epithelial ovarian cancer have primary metastatic disease with a corresponding 5-year survival of only $28 \%$ [1]. Therapeutic options for this advanced disease (International Federation of Gynecology and Obstetrics, FIGO stage IIB-IV) are either primary debulking surgery followed by adjuvant chemotherapy or induction chemotherapy followed by interval debulking surgery. The outcome of debulking surgery is by far the most important prognostic factor for patients with advanced ovarian cancer and surgery should always be aimed at achieving complete removal of macroscopic tumour [2; 3]. At the same time, recent investigations have led to the discovery that skeletal muscle area changes detected on computed tomography (CT) may be closely related to ovarian cancer survival as well $[4 ; 5]$. In our own ovarian cancer cohort of patients treated with induction chemotherapy and interval debulking surgery we have shown that patients who were able to gain or maintain muscle area during chemotherapy had a significantly better overall survival (OS) than patients who lost muscle area [4]. What became apparent in this study was that a measurement over time was essential to identify muscle loss or sarcopenia. A cross-sectional single time point measurement could not detect change and was thus unable to predict survival [4]. The importance of sarcopenia has scarcely been studied in ovarian cancer and these results have yet to be confirmed in international prospective trials. However, similar results have been found for other cancer types; stable or increasing muscle mass has been reported to relate to a prolonged survival in non-small cell lung cancer, pancreatic cancer and colorectal cancer while a low muscle mass at baseline showed no prognostic significance [6-8].

Cross-sectional CT measurement of the total skeletal muscle area (SMA) at the level of the third lumbar vertebra (L3) has proven to give a reliable representation of total body muscle mass and has therefore been adopted worldwide for the detection of sarcopenia in cancer patients $[9 ; 10]$. As an alternative for measuring all skeletal muscle visible at $\mathrm{L} 3$, one can opt to evaluate the psoas muscle alone. A scientific rationale for using the psoas is not provided by any authors using this muscle for evaluation of sarcopenia, but we speculate that it might have been selected due to ease of identification or possibly because of its functional role as a hip flexor muscle. In case of a decrease in weight-bearing exercise due to physical unfitness or hospitalisation, the psoas muscle is expected to decrease in volume, which can be used as a potential measure of muscle loss. Although imaging software is still needed, measuring the psoas area (PA) alone is easier and less time consuming. This method has been used to predict surgical complications in different cancer types with contrasting results. PA has shown a correlation with post-operative complications in individual studies on colorectal cancer, colorectal liver metastases, kidney cancer, bladder cancer, cholangiocarcinoma and hepatocellular carcinoma [11-16], while this effect was not seen in other pancreatic cancer, endometrial cancer, biliary cancer or sarcoma studies [17-20]. Interestingly, only 
few cancer studies were able to show a correlation between PA and survival [21-23]. In a small number of non-cancer studies, decreased psoas muscle area has also been correlated with higher rates of morbidity [24-26] and mortality [25; 27-29] in patients undergoing cardiothoracic, gastro-intestinal and spinal surgery. To simplify evaluation of muscle area, an even quicker and easier novel method has been reported. By multiplying the length and width of the psoas muscle (PLW), the psoas area can be directly calculated without the need for specialised software. Jones et al. studied 100 patients with colorectal cancer and reported a good correlation between the standard and new method to measure the psoas muscle as well as a good correlation between measurements of the SMA in comparison to the standard PA method [11].

Evaluation of change in muscle mass over time is important for ovarian cancer prognosis; patients who are identified adequately could possibly benefit from nutritional or physical interventions. Standard CT measurements of SMA have been able to detect changes and predict OS in patients with ovarian cancer undergoing induction chemotherapy and interval debulking. This study aims to investigate whether two methods to assess the psoas muscle area, PA and PWL, reflect the total muscle area and give a reliable representation of sarcopenia in ovarian cancer with the same accuracy as SMA assessment.

\section{METHODS}

This study has been approved by the local Medical Ethics Committee and has been performed in accordance with the ethical standards laid down in the 1964 Declaration of Helsinki. The requirement to obtain informed consent was waived by a Medical Ethics Committee.

\section{Eligible patients}

All patients with advanced ovarian cancer (FIGO 2013 stage IIB-IV) who were treated with induction chemotherapy and interval debulking in the Maastricht University Medical Centre (Maastricht, the Netherlands) between 2000 and 2015 were enrolled in this retrospective study. Patients were eligible for inclusion if they met the following criteria: 1 ) at least two routine abdominal CT scans were performed, the first before the start of induction chemotherapy and the second before interval debulking (typically after 3-4 cycles of chemotherapy), 2) the quality of both CT scans was sufficient to perform measurements of muscle area, 3) relevant clinical data could be retrieved from the patients' medical records, and 4) follow-up was at least 6 months after diagnosis. Part of this population was used in prior investigations from which the results have been published previously [4]. 
Patients were divided in age groups $\leq 60$ years, $61-70$ years and $>70$ years according to their age at diagnosis. Surgical outcome was categorised into complete (no visible evidence of macroscopic residual disease), optimal (macroscopic residual disease $\leq 1 \mathrm{~cm}$ ) or incomplete (macroscopic residual disease $>1 \mathrm{~cm}$ ). OS was defined as the period of time between the initial CT and a patient's death from any cause as reported in national registries. Patients who were still alive at the time of analysis were censored at a fixed date.

\section{CT analysis}

A single axial image corresponding to the L3 vertebral body was selected for each CT. For both SMA and PA, SliceOmatic software (v5.0, Tomovision, Montreal, QC, Canada) was used to quantify skeletal muscles within predefined validated boundaries of -29 to +150 Hounsfield Units. For SMA, the entire skeletal muscle area consisting of the abdominal muscles, psoas muscles and paraspinal muscles was demarcated. For PA only the psoas muscle area (right and left) was selected. Following demarcation, the surface areas were quantified automatically in $\mathrm{cm}^{2}$. For PLW, the length and width of the right and left psoas muscle was measured by hand and multiplied to compute the psoas surface area. Right and left psoas muscle were summated to estimate the total psoas length* width area in $\mathrm{cm}^{2}$. Two trained observers (IR and JU) blinded to patient details and clinical outcomes individually applied the three methods to all CT scans (Figure 1). Afterwards, measurements were averaged between the observers. Mean assessment time was recorded for a random sample of patients $(n=10)$ for each method. This assessment time only included the time in which the actual measurements were performed after the correct axial image at the level of $\mathrm{L} 3$ had already been selected and the right HU boundaries had been defined.

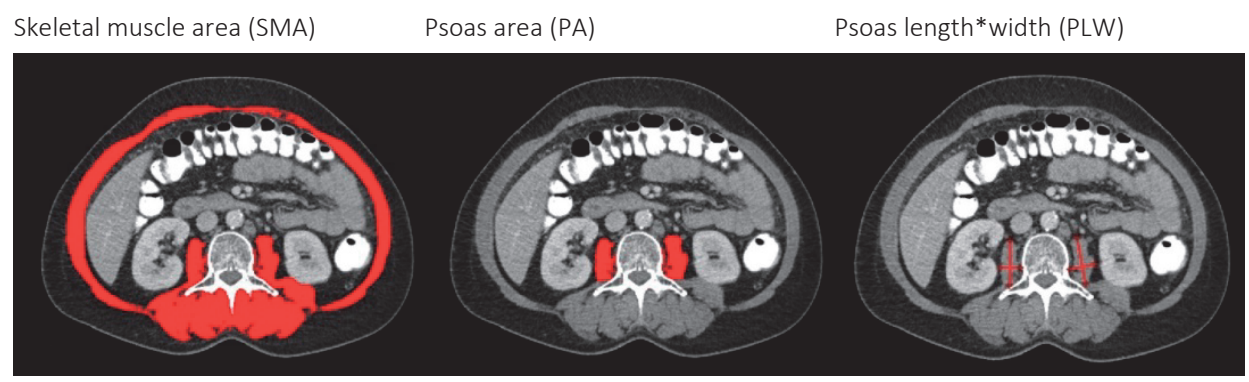

Fig. 1 Muscle area measurement methods

\section{Statistical analysis}

Interobserver correlation between observer 1 and 2 was evaluated for each method with the Pearson correlation coefficient $(r)$ and with the intraclass correlation 
coefficient (ICC). Pearson's $r$ measures linear correlation between two variables and equals 1 in case of perfect positive correlation, 0 in case of no correlation at all, and -1 in case of total negative correlation. ICC can be used to assess both consistency and absolute agreement between quantitative measurements made by multiple observers measuring the same quantity. Further statistical analyses were conducted with the averaged measurements between observers. Intermeasurement correlations between the three methods to measure muscle tissue were also studied with the Pearson correlation coefficient. Additionally a Bland-Altman plot was created to investigate the existence of any systematic differences between the two assay methods measuring psoas muscle area; PA and PLW. If the mean value of the difference between assay methods is significantly different from zero, this indicates a systematic difference between measurements.

The percentage change in muscle area between the pre- and post-chemotherapy CT scan was calculated per 100 days for each method. A measurement error of $2 \%$ was taken into account based on previously reported accuracy of CT for muscle analysis [9]. Patients were divided into two groups based on the extent of muscle area change: "Loss" in case of $>2 \%$ decrease per 100 days and "Gain" when any increase or $\leq 2 \%$ decrease was seen. Muscle area changes between $-2 \%$ and $+2 \%$ were considered as muscle stability and were included in the "Gain" group. Subsequently the three methods to quantify muscle area were compared categorically through the use of contingency tables. Cohen's Kappa coefficient $(\mathrm{K})$ was computed to measure agreement between SMA and PA, SMA and PLW, and PA and PLW. In case of perfect agreement, $\mathrm{K}$ gives a value of 1 .

Finally age, FIGO stage ("FIGO stage IV" versus "FIGO stage II and III combined"), surgical outcome ("complete" versus "incomplete and optimal combined") and muscle change determined by SMA, PA and PLW ("Ioss" versus "gain") were tested as effect modifiers in a univariable proportional hazards Cox-regression model at a significance level of $10 \%$. Hazard ratio's (HR) were calculated with $95 \%$ confidence intervals $(95 \% \mathrm{Cl})$. Using backwards stepwise elimination significant predictors were combined in a multivariable model in which a significance level of $5 \%$ was applied. All analyses were performed with the statistical software package SPSS v20.0 (IBM Corp, Chicago, IL).

\section{RESULTS}

\section{Baseline characteristics}

In total, 190 patients with advanced ovarian cancer treated with induction chemotherapy and interval debulking were identified. 40 patients were excluded due to unavailability or insufficient quality of one or both CT scans, due to missing clinical data or due to debulking being performed for recurrent disease. After exclusion, 150 patients 
and 300 CT scans were available for analysis. 123 of these 150 patients were used in previous investigations by our research group [4]. Patient characteristics are presented in Table 1. Median follow-up for censored patients was 904 days $(n=56)$ with a minimum of 209 days. Specification of muscle area change resulted in a median SMA loss of $5.8 \%$ per 100 days, a median PA gain of $1.4 \%$ per 100 days and a median PLW loss of $0.9 \%$ per 100 days. Mean assessment time was 110 seconds per patient, 27 seconds per patient and 16 seconds per patient for SMA, PA and PLW respectively.

Table 1 Patient Characteristics

\begin{tabular}{|c|c|}
\hline & All patients $(n=150)$ \\
\hline $\begin{array}{l}\text { Age in years, median } \pm \text { SD (range) } \\
\leq 60 \text { years, } n(\%) \\
61-70 \text { years, } n(\%) \\
>70 \text { years, } n(\%)\end{array}$ & $\begin{array}{l}67 \pm 9.8(39-86) \\
40(26.7) \\
51(34.0) \\
59(39.3)\end{array}$ \\
\hline $\begin{array}{l}\text { FIGO tumour stage } \\
\text { II, n (\%) } \\
\text { III, n (\%) } \\
\text { IV, n (\%) }\end{array}$ & $\begin{array}{l}2(1.3) \\
91(60.7) \\
57(38.0)\end{array}$ \\
\hline Days between CT scans, median \pm SD (range) & $82.5 \pm 22.4(47-190)$ \\
\hline $\begin{array}{l}\text { SMA in } \mathrm{cm}^{2} \\
\text { Pre-chemotherapy, median } \pm \text { SD } \\
\text { Post-chemotherapy, median } \pm \text { SD }\end{array}$ & $\begin{array}{l}110.2 \pm 15.4 \\
104.4 \pm 14.3\end{array}$ \\
\hline $\begin{array}{l}\text { PA in } \mathrm{cm}^{2} \\
\text { Pre-chemotherapy, median } \pm \text { SD } \\
\text { Post-chemotherapy, median } \pm \text { SD }\end{array}$ & $\begin{array}{l}13.3 \pm 3.1 \\
13.4 \pm 2.9\end{array}$ \\
\hline $\begin{array}{l}\text { PLW in } \mathrm{cm}^{2} \\
\text { Pre-chemotherapy, median } \pm \text { SD } \\
\text { Post-chemotherapy, median } \pm \text { SD }\end{array}$ & $\begin{array}{l}14.9 \pm 4.3 \\
14.5 \pm 4.0\end{array}$ \\
\hline $\begin{array}{l}\text { Muscle area changes in } \% \text { change per } 100 \text { days } \\
\text { SMA, median } \pm \text { SD } \\
\text { PA, median } \pm \text { SD } \\
\text { PLW, median } \pm \text { SD }\end{array}$ & $\begin{array}{l}-5.8 \pm 9.9 \\
+1.4 \pm 21.1 \\
-0.9 \pm 15.7\end{array}$ \\
\hline $\begin{array}{l}\text { Outcome interval debulking } \\
\text { Complete, } \mathrm{n}(\%) \\
\text { Optimal, } \mathrm{n}(\%) \\
\text { Incomplete, } \mathrm{n}(\%)\end{array}$ & $\begin{array}{l}69(46.0) \\
55(36.7) \\
26(17.3)\end{array}$ \\
\hline OS in days, median \pm SD & $711 \pm 753$ \\
\hline
\end{tabular}

SD, standard deviation; FIGO, International Federation of Gynaecology and Obstetrics; SMA, skeletal muscle area; PA, psoas area; PLW, psoas length*width; OS, overall survival

\section{Interobserver and intermeasurement correlations}

Interobserver correlation results for the different measurements on the prechemotherapy CT scan are given in Figure 2. For assessment of SMA and PA, agreement was almost perfect with Pearson's $r$ values of 0.96 and 0.99 respectively. Interobserver agreement for PLW was 0.85 . Mean SM measured by observer 1 was 110.7 with a 


\section{Chapter 4}

standard deviaton (SD) of 15.4 and mean SM measured by observer 2 was 112.2, SD 15.8. Mean PA was 13.7 (SD 3.2) for observer 1 and 13.9 (SD 3.1) for observer 2. Mean PLW was 15.9 (SD 4.6) for observer 1 and 14.4 (SD 4.3) for observer 2. ICC assessing consistency and absolute agreement between observers was 0.96 and 0.96 for SM, 0.99 and 0.99 for PA and 0.85 and 0.80 for PLW respectively. Interobserver agreement was also measured for post chemotherapy scans which resulted in similar Pearson's $r$ values of $0.99,0.98$ and 0.80 for SMA, PA and PLW respectively.
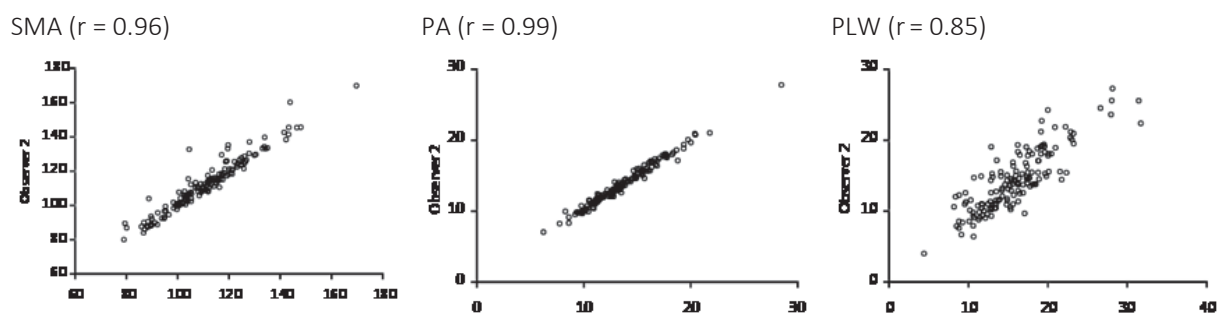

Fig. 2 Interobserver correlation, analysis with data from pre-chemotherapy CT scan SMA, skeletal muscle area; PA, psoas area; PLW, psoas length*width

SMA vs. PA $(r=0.52)$

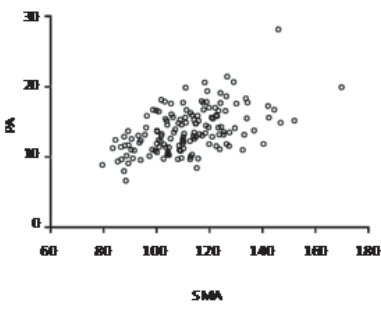

SMA vs. PLW $(r=0.39)$

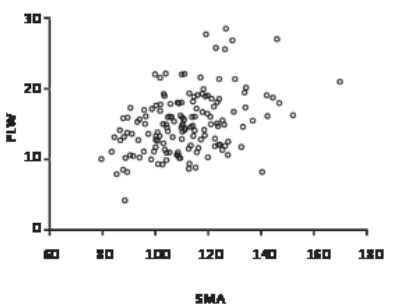

PA vs. PLW ( $r=0.83)$

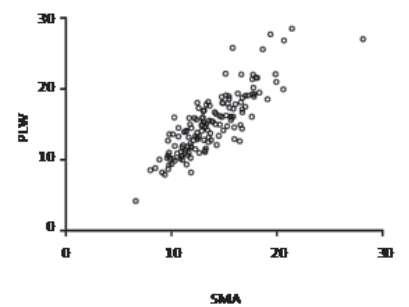

Fig. 3 Intermeasurement correlation, analysis with data from pre-chemotherapy CT scan SMA, skeletal muscle area; PA, psoas area; PLW, psoas length*width

Intermeasurement correlation between SMA and PA was 0.52 and 0.56 for pre- and post-chemotherapy scans respectively. Correlation between SMA and PLW was 0.39 and 0.44 for pre- and post-chemotherapy scans respectively. Correlation between PA and PLW was 0.83 and 0.84 for pre- and post-chemotherapy scans respectively. Scatter plots for correlation between the methods applied to the pre-chemotherapy scan are shown in Figure 3. The mean difference between PLW and PA measuring psoas muscle on the pre-chemotherapy scan was 1.35 with a standard deviation of 2.33 , which was significantly different from zero $(p<0.001)$ and indicates that the two assay methods are systematically producing different results. The corresponding Bland-Altman plot created with 95\% confidence intervals is shown in Figure 4. 


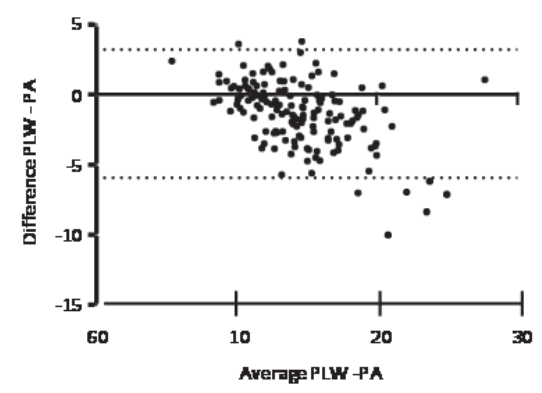

Fig. 4 Bland-Altman plot comparing PLW and PA for measurement of psoas muscle area, analysis with data from pre-chemotherapy CT scan.

PLW, psoas length*width; PA, psoas area

Contingency tables in which patients were categorised into loss or gain are shown in Table 2. Cohen's k was 0.182 for SMA and PA, 0.312 for SMA and PLW and 0.226 for PA and PLW. Discrepancies were found in $44 \%(n=66)$ of cases when comparing SMA and PA. In the comparison of SMA and PLW, 35\% $(n=53)$ discrepant cases were found and PA and PLW disagreed in 38\% $(n=57)$ of cases.

Table 2 Contingency tables

\begin{tabular}{|c|c|c|c|c|c|c|c|c|c|c|c|}
\hline \multicolumn{4}{|c|}{ SMA vs. PA ( $k=0.182)$} & \multicolumn{4}{|c|}{ SMA vs. PLW ( $k=0.312)$} & \multicolumn{4}{|c|}{ PA vs. PLW $(k=0.226)$} \\
\hline & \multicolumn{3}{|l|}{ PA } & \multicolumn{4}{|c|}{ PLW } & \multicolumn{4}{|c|}{ PLW } \\
\hline & Loss & Gain & Total & & Loss & Gain & Total & & Loss & Gain & Total \\
\hline Loss & 46 & 54 & 100 & Loss & 58 & 42 & 100 & Loss & 35 & 23 & 58 \\
\hline$\varangle_{\text {Gain }}$ & 12 & 38 & 50 & $\varangle_{\text {Gain }}$ & 11 & 39 & 50 & Gain & 34 & 58 & 92 \\
\hline$\sum_{n}^{\infty}$ Total & 58 & 92 & 150 & $\sum_{n}^{\infty}$ Total & 69 & 81 & 150 & $\llbracket$ Total & 69 & 81 & 150 \\
\hline
\end{tabular}

SMA, skeletal muscle area; PA, psoas area; PLW, psoas length*width

\section{Survival analysis}

Age, FIGO stage IV, complete interval debulking and muscle loss measured with SMA were significant predictors of OS at a significance level of $10 \%$ in univariable Coxregression analysis (Table 3 ). No relationship with OS was seen for measurement of psoas muscle loss with PA nor with PLW. In multivariable analysis FIGO stage IV (HR $1.730(95 \% \mathrm{Cl} 1.129-2.652), p=0.012)$, complete interval debulking ( $\mathrm{HR} 0.381(95 \% \mathrm{Cl}$ 0.246-0.589), $p<0.001)$ and loss of SMA (HR $1.698(95 \% \mathrm{Cl} 1.038-2.778), p=0.035)$ were predictive of OS. Median OS was 665 days for patients who lost SMA compared to 914 days for patients who maintained or gained SMA ( $p=0.017)$. 


\section{Chapter 4}

Table 3 Univariable and multivariable Cox-regression analysis

\begin{tabular}{|c|c|c|c|c|}
\hline \multirow[b]{2}{*}{ Variables } & \multicolumn{2}{|l|}{ Univariable analysis } & \multicolumn{2}{|l|}{ Multivariable analysis } \\
\hline & $\mathrm{HR}(95 \% \mathrm{CI})$ & p-value & $\mathrm{HR}(95 \% \mathrm{Cl})$ & p-value \\
\hline Age & $1.026(1.002-1.050)$ & $0.032^{*}$ & - & - \\
\hline FIGO tumour stage IV & $1.489(0.980-2.262)$ & $0.062^{*}$ & $1.730(1.129-2.652)$ & $0.012^{*}$ \\
\hline Complete interval debulking & $0.408(0.259-0.643)$ & $<0.001^{*}$ & $0.381(0.246-0.589)$ & $<0.001^{*}$ \\
\hline Muscle loss - SMA & $2.069(1.285-3.332)$ & $0.003^{*}$ & $1.698(1.038-2.778)$ & $0.035^{*}$ \\
\hline Muscle loss - PA & $0.979(0.643-1.491)$ & 0.921 & - & - \\
\hline Muscle loss - PLW & $1.101(0.730-1.662)$ & 0.645 & - & - \\
\hline
\end{tabular}

$\mathrm{HR}$, hazard ratio; $\mathrm{Cl}$, confidence interval; FIGO, International Federation of Gynaecology and Obstetrics; SMA, skeletal muscle area; PA, psoas area; PLW, psoas length*width.

* Indicates significant $p$-value ( $p<0.10$ for univariable analysis and $p<0.05$ for multivariable analysis).

\section{DISCUSSION}

\section{Key findings}

The objective of this study was to investigate whether assessment of psoas muscle area reflects total muscle area and can be used to assess sarcopenia in ovarian cancer patients undergoing induction chemotherapy and interval debulking. Two different quantification methods of the psoas muscle area were compared to the reference method of measuring total skeletal muscle area. We found a weak correlation between SMA and PA measurements and an even weaker correlation between SMA and PLW. When categorising the findings into muscle area loss or gain, high rates of discrepancies were also found between both SMA and PA or PLW. The correlation between the two assessment methods of psoas area was reasonable but also resulted in a high number of discrepancies when categorising patients. From these data we can conclude that measurement of psoas muscle area either with software delineation of surface area or with manual measurement of length and width does not give a reliable representation of skeletal muscle loss when compared to measurement of total skeletal muscle mass. The regression analysis confirmed these results; PA and PLW were not helpful in the prediction of OS whereas SMA proved to be an important individual factor in both univariable and multivariable Cox regression-analysis corrected for age, tumour stage and surgical outcome. A clear difference in median OS was seen between patients who lost SMA and patients who maintained or gained SMA (665 days versus 914 days).

Many studies have used PA instead of SMA for assessment of sarcopenia in various cancer types. Although in some studies a correlation between PA and post-operative complications was seen, the majority have failed to prove a relationship between PAassessed sarcopenia and survival [11-23]. Only few have actually assessed the agreement between PA and SMA within their population; Jones et al. studied 100 
patients with colorectal cancer and reported a Spearman correlation of 0.8 for PA and SMA and a Spearman correlation of 0.94 for PA and PLW, which could not be reproduced in our cohort [11]. A possible explanation for this discrepancy might be the difference in statistical analysis. Whereas we used the Pearson correlation coefficient, Jones et al. used Spearman's rank correlation coefficient. Pearson's method is used for linear relationships and was applicable to our data while Spearman's method can be applied to nonparametric data. The outcome produced by the two methods can vary according to the character of the data. It is unclear why the authors chose to use Spearman instead of Pearson correlation. However, when we tested Spearman's correlation in our data, results were not different from what we found with Pearson's correlation and they were again not comparable to the high correlation found in Jones' study. Another plausible difference between our studies is the software that was used: ImageJ versus SliceOmatic. However since both software programs measure and quantify tissue by outlining the muscle area and similar measurements are expected by both methods, this does not fully explain the difference in intermeasurement correlation. In addition to the former study, Taguchi et al. found a Spearman correlation of 0.75 for PA and SMA in 64 patients with urothelial carcinoma which was also markedly higher than the Pearson correlation of 0.52 we found in our population [30]. In this study a slightly different method of assessing PLW was used in which the length and width of the psoas muscle were compared separately and not as a combined measure. The reported Spearman correlation of 0.81 for PA and psoas width was comparable to our reported Pearson correlation of 0.83 for PA and PLW.

We believe that L3 psoas muscle area measurements are not representative of total L3 skeletal muscle area. A plausible reason why the psoas muscle is less representative than the total muscle at $\mathrm{L} 3$ is that the psoas muscle is prone to be focally affected by degenerative diseases of the lumbar spine. Lumbar degenerative disc and facet joint disease can cause local atrophy of the trunk muscles and psoas muscle loss is hence not specifically related to cancer-induced sarcopenia [31]. Psoas analysis should therefore not be conducted on patients with a medical history of spine surgery, lower back pain, degenerative lumbar instability, vertebral fracture, and deformity. This has a massive impact when studying cancer populations. First, medical records of all patients need to be evaluated to confirm which individuals are affected by the above conditions and, subsequently, will have to be excluded from the analysis. Secondly, degenerative diseases of the lumbar spine are rather prevalent in older patients, and in cancer populations (the mean age in our study population was $>65$ years). This would result in the psoas method being inapplicable in large numbers of individuals and especially elderly. Although the psoas muscle is a hip flexor muscle which could be expected to give a representation of physical fitness, the PA only represents $10 \%$ of the SMA measurable at L3. By using the PA alone you ignore vital information about the remaining skeletal muscles. Because the $\mathrm{PA}$ is so much smaller in comparison to the SMA, it is also much less sensitive to depict change. In our opinion, measuring change 
over time is the most accurate way to portray sarcopenia in ovarian cancer and therefore we believe that PA should not be used to substitute SMA to predict survival. The rates of muscle loss and gain we found seem to be consistent throughout the 15year period of time that we studied since we have not noticed specific outliers within a certain time period.

Our results have shown an exceedingly well interobserver agreement with correlation coefficients between 0.96 and 0.99 for assessment of SMA and PA with SliceOmatic software. When comparing the results for Pearson's correlation and intraclass correlation we find almost identical results, which is suggestive for both a high consistency and high agreement between observers. A similar interobserver agreement $(r=0.97)$ was found by Jones et al. for the evaluation of PA [11]. Agreement between observers was less strong for the manual technique measuring PLW ( $r=0.80-0.85)$. This may be explained by the fact that the length and width of the psoas muscle are difficult to measure due to the great variation in shape of the psoas muscle. The psoas muscle is only a small muscle; therefore any small errors in measurement may extrapolate to larger errors in rate of change when multiplying length and width in PLW measurements, contributing to a lower interobserver agreement. Additionally, when assessing psoas muscle with PLW, the intramuscular adipose tissue is included in the measurement which gives an overestimation of the actual muscle tissue. In the pathophysiology of (cancer) cachexia, skeletal muscle may be replaced by intramuscular adipose tissue. In this way, both the quantity and the quality of muscle is being influenced. Linear methods of assessing skeletal muscle such as PLW measurements cannot depict these important changes. Software delineation using predefined HU boundaries can take the intramuscular adipose tissue into account and therefore reflects a more accurate quantity of skeletal muscle. The overestimation of muscle surface area with a linear method is reflected in Table 1 where we find a higher mean PLW in comparison to mean PA.

\section{Limitations}

Due to the retrospective nature of this research our analyses were to some extent restricted by missing data. Due to several irretrievable CT scans, a number of patients were excluded from the analysis. Also, by limiting our study population to patients who were treated with induction chemotherapy and interval debulking and excluding patients treated with primary debulking we have created a selection bias. Effects on muscle mass might become more apparent in the population treated with induction chemotherapy which is prone to have more advanced tumour spread and/or a worse performance status. Whether evaluation of PA or muscle area estimation in general has any importance in ovarian cancer patients who receive primary debulking surgery without induction chemotherapy is unclear. Previous studies in other cancer populations have mainly focused on the relationship between PA and surgical 
complications and used only one CT measurement. Ovarian cancer patients selected for primary surgical treatment are also subjected to only one clinical CT scan and it would be interesting to see if an association can be found between PA and complications in this group.

Part of our study population was used in previous investigations from which the results have been published recently [4]. In this manuscript we concluded that SMA loss was predictive of OS, which was confirmed in the current study. However the additional value of this finding may be limited due to the overlap in patients studied ( $82 \%$ overlap). External validation of these findings in patients with other gynaecological malignancies and ovarian cancer specifically is imperative. Comparable results have been reported for patients with lung cancer, pancreatic cancer and colorectal cancer but these populations were primarily comprised of male patients and translation of these results to female patients - with lower muscle mass in general - should be applied carefully [6-8].

\section{Conclusion and implications for practice and research}

Change in psoas muscle area is not representative of total muscle area change and should not be used to substitute total skeletal muscle to predict survival in patients with ovarian cancer undergoing induction chemotherapy and interval debulking. Assessment of psoas muscle area may be easier and quicker, but is less sensitive to muscle change than standard assessment of total skeletal muscle. Measuring cross-sectional total skeletal muscle area at L3 showed strong interobserver agreement and has proven to be a significant predictor for OS and should therefore not be substituted by psoas area evaluation alone.

This study does not answer the important question why some patients with ovarian cancer lose while others gain muscle mass. The present study underpins the observation that sarcopenia is a problem in patients with ovarian cancer and that it has a substantial effect on survival. External validation of our findings is crucial and may lead to prospective intervention trials investigating how prevention of muscle loss can improve prognosis of patients with ovarian cancer. 


\section{REFERENCES}

1 Surveillance E, and End Results (SEER) Program SEER 9 Incidence \& U.S. Mortality Research Data (19732011). In: National Cancer Institute D, Surveillance Research Program, Surveillance Systems Branch, released April 2014, based on the November 2013 submission, (ed),

2 du Bois A, Reuss A, Pujade-Lauraine E, Harter P, Ray-Coquard I, Pfisterer J (2009) Role of surgical outcome as prognostic factor in advanced epithelial ovarian cancer: a combined exploratory analysis of 3 prospectively randomized phase 3 multicenter trials: by the Arbeitsgemeinschaft Gynaekologische Onkologie Studiengruppe Ovarialkarzinom (AGO-OVAR) and the Groupe d'Investigateurs Nationaux Pour les Etudes des Cancers de I'Ovaire (GINECO). Cancer 115:1234-1244

3 Vergote I, Trope CG, Amant F et al (2010) Neoadjuvant chemotherapy or primary surgery in stage IIIC or IV ovarian cancer. N Engl J Med 363:943-953

4 Rutten IJG, Van Dijk DPJ, Kruitwagen RFPM, Beets-Tan RGH, Olde Damink SW, Van Gorp T (2016) Loss of skeletal muscle during neoadjuvant chemotherapy is related to decreased survival in ovarian cancer patients. Journal of Cachexia, Sarcopenia and Muscle. 10.1002/jcsm.12107

5 Aust S, Knogler T, Pils D et al (2015) Skeletal Muscle Depletion and Markers for Cancer Cachexia Are Strong Prognostic Factors in Epithelial Ovarian Cancer. PLoS One 10:e0140403

6 Choi Y, Oh DY, Kim TY et al (2015) Skeletal Muscle Depletion Predicts the Prognosis of Patients with Advanced Pancreatic Cancer Undergoing Palliative Chemotherapy, Independent of Body Mass Index. PLoS One 10:e0139749

7 Miyamoto Y, Baba Y, Sakamoto Y et al (2015) Negative Impact of Skeletal Muscle Loss after Systemic Chemotherapy in Patients with Unresectable Colorectal Cancer. PLoS One 10:e0129742

8 Stene GB, Helbostad JL, Amundsen T et al (2015) Changes in skeletal muscle mass during palliative chemotherapy in patients with advanced lung cancer. Acta Oncol 54:340-348

9 Mourtzakis M, Prado CM, Lieffers JR, Reiman T, McCargar LJ, Baracos VE (2008) A practical and precise approach to quantification of body composition in cancer patients using computed tomography images acquired during routine care. Appl Physiol Nutr Metab 33:997-1006

10 Shen W, Punyanitya M, Wang Z et al (2004) Total body skeletal muscle and adipose tissue volumes: estimation from a single abdominal cross-sectional image. J Appl Physiol (1985) 97:2333-2338

11 Jones KI, Doleman B, Scott S, Lund JN, Williams JP (2015) Simple psoas cross-sectional area measurement is a quick and easy method to assess sarcopenia and predicts major surgical complications. Colorectal Dis 17:020-26

12 Peng PD, van Vledder MG, Tsai S et al (2011) Sarcopenia negatively impacts short-term outcomes in patients undergoing hepatic resection for colorectal liver metastasis. HPB (Oxford) 13:439-446

13 Peyton CC, Heavner MG, Rague JT, Krane LS, Hemal AK (2016) Does Sarcopenia Impact Complications and Overall Survival in Patients Undergoing Radical Nephrectomy for Stage III and IV Kidney Cancer? J Endourol 30:229-236

14 Smith AB, Deal AM, Yu H et al (2014) Sarcopenia as a predictor of complications and survival following radical cystectomy. J Urol 191:1714-1720

15 Valero V, 3rd, Amini N, Spolverato G et al (2015) Sarcopenia adversely impacts postoperative complications following resection or transplantation in patients with primary liver tumors. J Gastrointest Surg 19:272-281

16 Otsuji H, Yokoyama Y, Ebata T et al (2015) Preoperative sarcopenia negatively impacts postoperative outcomes following major hepatectomy with extrahepatic bile duct resection. World J Surg 39:14941500

17 Amini N, Spolverato G, Gupta R et al (2015) Impact Total Psoas Volume on Short- and Long-Term Outcomes in Patients Undergoing Curative Resection for Pancreatic Adenocarcinoma: a New Tool to Assess Sarcopenia. J Gastrointest Surg 19:1593-1602

18 Kuroki LM, Mangano M, Allsworth JE et al (2015) Pre-operative Assessment of Muscle Mass to Predict Surgical Complications and Prognosis in Patients With Endometrial Cancer. Ann Surg Oncol 22:972-979 
19 Okumura S, Kaido T, Hamaguchi Y et al (2015) Impact of the preoperative quantity and quality of skeletal muscle on outcomes after resection of extrahepatic biliary malignancies. Surgery. 10.1016/j.surg. 2015.08.047

20 Wilson RJ, Alamanda VK, Hartley KG et al (2015) Sarcopenia Does Not Affect Survival or Outcomes in Soft-Tissue Sarcoma. Sarcoma 2015:146481

21 Okumura S, Kaido T, Hamaguchi Y et al (2015) Impact of preoperative quality as well as quantity of skeletal muscle on survival after resection of pancreatic cancer. Surgery 157:1088-1098

22 Jung HW, Kim JW, Kim JY et al (2015) Effect of muscle mass on toxicity and survival in patients with colon cancer undergoing adjuvant chemotherapy. Support Care Cancer 23:687-694

23 Miller BS, Ignatoski KM, Daignault S et al (2012) Worsening central sarcopenia and increasing intraabdominal fat correlate with decreased survival in patients with adrenocortical carcinoma. World J Surg 36:1509-1516

24 Fujikawa H, Araki T, Okita Y et al (2016) Impact of sarcopenia on surgical site infection after restorative proctocolectomy for ulcerative colitis. Surg Today. 10.1007/s00595-016-1357-x

25 Masuda T, Shirabe K, Ikegami T et al (2014) Sarcopenia is a prognostic factor in living donor liver transplantation. Liver Transpl 20:401-407

26 Zakaria HM, Schultz L, Mossa-Basha F, Griffith B, Chang V (2015) Morphometrics as a predictor of perioperative morbidity after lumbar spine surgery. Neurosurg Focus 39:E5

27 Mamane S, Mullie L, Piazza N et al (2016) Psoas Muscle Area and All-Cause Mortality After Transcatheter Aortic Valve Replacement: The Montreal-Munich Study. Can J Cardiol 32:177-182

28 Onesti JK, Wright GP, Kenning SE et al (2016) Sarcopenia and survival in patients undergoing pancreatic resection. Pancreatology 16:284-289

29 Saji M, Lim DS, Ragosta M et al (2016) Usefulness of Psoas Muscle Area to Predict Mortality in Patients Undergoing Transcatheter Aortic Valve Replacement. Am J Cardiol 118:251-257

30 Taguchi S, Akamatsu N, Nakagawa T et al (2015) Sarcopenia Evaluated Using the Skeletal Muscle Index IS a Significant Prognostic Factor for Metastatic Urothelial Carcinoma. Clin Genitourin Cancer. 10.1016/j.clgc.2015.07.015

31 Sebro R, O'Brien L, Torriani M, Bredella MA (2016) Assessment of trunk muscle density using CT and its association with degenerative disc and facet joint disease of the lumbar spine. Skeletal Radiol 45:12211226 

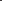


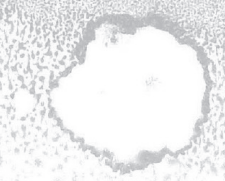

Chapter

\section{Prediction of incomplete primary debulking surgery in patients with advanced ovarian cancer:} An external validation study of three models using computed tomography 


\section{Chapter 5}

\section{ABSTRACT}

Objective: To test the ability of three prospectively developed computed tomography (CT) models to predict incomplete primary debulking surgery in patients with advanced (International Federation of Gynecology and Obstetrics stage III-IV) ovarian cancer.

Methods: Three prediction models to predict incomplete surgery (any tumor residual $>1$ $\mathrm{cm}$ in diameter) previously published by Ferrandina (models A and B) and by Gerestein were applied to a validation cohort consisting of 151 patients with advanced epithelial ovarian cancer. All patients were treated with primary debulking surgery in the Eastern part of the Netherlands between 2000 and 2009 and data were retrospectively collected. Three individual readers evaluated the radiographic parameters and gave a subjective assessment. Using the predicted probabilities from the models, the Area Under the Curve (AUC) was calculated which represents the discriminative ability of the model.

Results: The AUC of the Ferrandina models was $0.56,0.59$ and 0.59 in model $A$, and $0.55,0.60$ and 0.59 in model B for readers 1,2 and 3, respectively. The AUC of Gerestein's model was 0.69, 0.61 and 0.69 for readers 1, 2 and 3, respectively. AUC values of 0.69 and 0.63 for reader 1 and 3 were found for subjective assessment.

Conclusions: Models to predict incomplete surgery in advanced ovarian cancer have limited predictive ability and their reproducibility is questionable. Subjective assessment seems as successful as applying predictive models. Present prediction models are not reliable enough to be used in clinical decision-making and should be interpreted with caution. 


\section{INTRODUCTION}

Ovarian cancer has a high mortality which translates to a 5-year survival of $38-46 \%$ in Europe and the United States [1; 2]. These survival rates drop from 73-90\% in early stage epithelial ovarian cancer to $17-39 \%$ in advanced stages (International Federation of Gynecology and Obstetrics, (FIGO) stage III and IV) [2]. The most important predictor of survival in advanced stage disease is the amount of residual tumor after cytoreductive surgery [3-5]. Maximal cytoreduction has been the mainstay of advanced ovarian cancer treatment for many years now since Griffiths et al established the inverse relationship between the amount of residual disease and overall survival in 1975 [6]. Multiple studies have consistently confirmed these findings and more recent data demonstrate a significant survival gain for complete resection to no macroscopic residual disease in comparison to optimal resection to a tumor residual of $\leq 1 \mathrm{~cm}[4 ; 5]$. Incomplete resection (tumor residual $>1 \mathrm{~cm}$ ) has little beneficial effects for survival while it can cause substantial perioperative morbidity. It is widely agreed that surgery should be avoided when incomplete resection is expected. Neoadjuvant chemotherapy followed by interval debulking surgery provides a good treatment alternative with similar survival rates when primary surgery is deemed not feasible or impossible [3; 7].

Whether or not complete tumor resection can be achieved depends on patient related factors such as age and comorbidity, the extent and location of disease and the skill and experience of the operating surgeon [8]. Numerous prediction models have been developed in order to select patients with advanced ovarian cancer who are unlikely to benefit from primary debulking surgery [9-24]. They have incorporated clinical features and computed tomography (CT) features in different combinations. Frequently used CT predictors are peritoneal thickening, ascites, suprarenal lymphadenopathy, involvement of the bowel mesentery, diaphragmatic involvement and liver involvement [25]. Recurring clinical features included in the prediction models are serum Ca-125 levels and World Health Organization Performance Status (WHO-PS) [25].

Agreement between the developed models is limited and the great variety of combinations of predictors found to be associated with ovarian cancer resectability makes the reproducibility and clinical applicability of the models questionable. Many of the earlier models defined optimal debulking as $<2 \mathrm{~cm}$ residual disease instead of $\leq 1$ cm [9-12] or included early stage ovarian cancer [10; 11; 13; 14]. Furthermore most studies, including many of the newer models, were developed with retrospective data from small populations and were only validated internally or not validated at all. External validation in a population other than the study population is mandatory before a model can be implemented in daily practice. Two studies tested the accuracy of the models developed by Bristow et al [15] and Dowdy et al [16] and found a substantial decline in predictive performance when the prediction models were tested on a different population [26; 27]. Since then more models have been developed but few have been tested for external validity [17-24]. 


\section{Chapter 5}

The goal of this study is to externally validate three prospectively developed CT models published by Ferrandina et al [17] and by Gerestein et al [18] to predict incomplete debulking surgery in advanced stage ovarian cancer patients undergoing primary debulking surgery. We aim to apply the three models on our validation cohort and test their accuracy when interpreted by different readers.

\section{METHODS}

\section{Selection of patients}

A Medical Ethical Committee approved this study and waived the requirement for obtaining informed consent. All patients diagnosed with primary advanced (FIGO stage III and IV) epithelial ovarian cancer that were treated in the Eastern part of the Netherlands (one specialised academic centre and six regional hospitals) between 1 January 2000 and 1 January 2009 were identified. Patients were included if they fulfilled the following criteria: 1) patients were treated with primary debulking surgery and 2) a preoperative CT scan was performed within three months before surgical treatment. Data on patient characteristics, diagnosis and therapy were retrospectively collected from local hospital records. Collection of imaging data was also done retrospectively. Patients were excluded in case of missing data regarding surgical outcome or when the preoperative CT scan could not be retrieved. None of the included patients were used in the development of any of the models under evaluation and as such, this population represents an independent external validation cohort.

\section{Prediction models}

The prediction models under evaluation in this study were constructed by Ferrandina et al in 2009 and Gerestein et al in 2011 [17; 18].

Ferrandina described two approaches to predict incomplete primary debulking in advanced ovarian cancer: model $A$ and $B$. In model $A$, each radiographic or clinical parameter that showed a specificity $\geq 75 \%$, a positive predictive value (PPV) $\geq 50 \%$, and a negative predictive value (NPV) $\geq 50 \%$ in predicting surgical outcome was assigned a score of 1 point. In addition, if a parameter showed an overall accuracy $\geq 60 \%$, it was assigned a second point. In total, 4 radiographic parameters were assigned 1 or 2 points and the clinical parameter WHO-PS was assigned 2 points in the final model as well (see Table 1 ). A cut-off of more than 5 points out of a possible 8 point score was used to predict incomplete debulking. In model $\mathrm{B}$, the relationship between each possible predictor with surgical outcome was tested in univariable logistic regression analysis at a significance level of 5\%. Significant predictors were included in a multivariable logistic regression model using a stepwise elimination method. All predictors achieving a $p$-value $<0.10$ were 
assigned a score of 1 point. The final model included 4 radiographic parameters and 1 clinical parameter. The parameters are specified in Table 1 . A cut-off of $>3$ points out of a possible 5 point score was used to predict incomplete debulking. Reported Area Under the Curve (AUC) values were 0.81 for model $A$ and 0.82 for model $B$.

Table 1. Parameters included in the prediction models

\begin{tabular}{|c|c|c|c|c|}
\hline Ferrandina - Model A & Points & Ferrandina - Model B & Points & Gerestein \\
\hline Radiographic & & Radiographic & & Radiographic \\
\hline $\begin{array}{l}\text { Bowel mesentery } \\
\text { involvement }\end{array}$ & 2 & $\begin{array}{l}\text { Bowel mesentery } \\
\text { involvement }\end{array}$ & 1 & $\begin{array}{l}\text { Diffuse peritoneal } \\
\text { thickening }\end{array}$ \\
\hline Diaphragmatic disease & 2 & Diaphragmatic disease & 1 & Large volume ascites \\
\hline Liver involvement & 1 & $\begin{array}{l}\text { Suprarenal aortic lymph } \\
\text { nodes }\end{array}$ & 1 & \\
\hline Omental extension & 1 & $\begin{array}{l}\text { Peritoneal thickening } \\
\text { and/or implants }\end{array}$ & 1 & \\
\hline Clinical & & Clinical & & Clinical \\
\hline WHO-PS (0-1 vs. 2) & 2 & WHO-PS (0-1 vs. 2) & 1 & Blood platelet count \\
\hline
\end{tabular}

WHO-PS $=$ World Health Organization Performance Status

Gerestein performed univariable regression analysis at a significance level of $30 \%$ to select parameters that were associated with surgical outcome in advanced ovarian cancer patients. The selected parameters were included in a multivariable Cox regression model using a backward elimination method. Two radiographic predictors and one clinical predictor were included in the final prediction model (see Table 1). The model was internally validated by a bootstrap method which resulted in a c-index of 0.67 .

\section{Data analysis}

CT scans from patients in our external validation cohort were assessed in a retrospective manner by three radiologists from different hospitals (two academic centres and one regional hospital). All readers had a special interest in gynecologic oncology imaging and had eight years (FB) or four years (MP and TP) of experience in this field. For their assessment the readers individually scored the radiographic parameters included in the original prediction models by Ferrandina and Gerestein blinded to any clinical information and surgical outcome. Two of the three radiologists (FB, TP) were also asked to give their own subjective judgement whether incomplete surgery was expected. Clinical parameters were retrieved from hospital records. All patients underwent laparotomy aimed at achieving maximal cytoreduction according to prevailing guidelines. Incomplete tumor resection was defined as any tumor residual greater than $1 \mathrm{~cm}$ in diameter. Surgical findings and histopathological confirmation were used as reference standard. 


\section{Statistical analysis}

First, Ferrandina's prediction models $A$ and $B$ were applied to the validation cohort. Using the described cut-off values of 5 in model $A$ and 3 in model $B$, the sensitivity, specificity, PPV, NPV, pre-test probability and post-test probability were calculated. A true positive (TP) was defined as a patient in whom an incomplete debulking was correctly predicted (score $>5$ or $>3$ ). A true negative (TN) was defined as a patient in whom a complete or optimal debulking was correctly predicted (score $<5$ or $<3$ ). The pre-test probability is the portion of patients who received incomplete surgery in relation to the total group of patients (=prevalence of incomplete debulking surgeries). The post-test probability is calculated by dividing the number of TPs by the total number of patients with a positive test score. Using the predicted probabilities from the models for all observed cut-off values, a receiver operating characteristic (ROC) curve was generated. The Area Under the Curve (AUC), which is calculated from the ROC curve, represents the concordance index (c-index). The c-index expresses the ability of the model to predict surgical outcome thus discriminating between patients with incomplete resection and patients with complete or optimal resection. This is achieved by quantifying the amount of concordance between the predicted probabilities from the model and the actual chance of having incomplete surgery. Since Gerestein's model did not include a point scale, the predictive performance of the model could not be expressed in sensitivity or specificity. Instead, the AUC value was used to indicate the discriminative ability of the model. The AUC was calculated by using the predicted probabilities from the multivariable logistic regression analysis in a similar way as mentioned above. Both Ferrandina models and Gerestein's model were tested for each reader separately.

Additionally, we assessed the individual radiographic and clinical parameters from the validated models in a logistic regression analysis. Parameters with a p-value $<0.10$ in univariable analysis were included in a multivariable logistic regression model using a backwards stepwise elimination method in which a $p$-value of $<0.05$ was considered significant. Three analyses were performed in this manner, one for each reader.

Interobserver variability was indicated by kappa values and was measured for each scored radiographic measure among the three readers as well as for subjective assessment between readers 1 and 3. A kappa value of 0.01-0.20 implied slight agreement, $0.21-0.40$ fair agreement, $0.41-0.60$ moderate agreement, $0.61-0.80$ substantial agreement and a kappa of 0.81-0.99 signified almost perfect agreement.

Finally, the diagnostic performance of subjective assessment alone was expressed in terms of sensitivity, specificity, accuracy and AUC for readers 1 and 3. Data on subjective assessment were not available for reader 2 . 


\section{RESULTS}

\section{Study population}

In total, 180 patients with advanced stage ovarian cancer who underwent preoperative CT imaging and subsequent primary debulking surgery were eligible for inclusion. Seven patients were excluded due to missing data on surgical outcome and another 22 patients were excluded because the preoperative CT scan could not be retrieved. Finally, our validation cohort consisted of 151 patients with a median age of 63 years (range 30-88). Eighty-six percent of patients received surgical treatment within one month from preoperative imaging. Patient characteristics from the current study cohort and the original cohorts of Ferrandina and Gerestein are given in Table 2. Patient age was comparable between the three cohorts with a median age of 59 years in Ferrandina's cohort, 62 years in Gerestein's cohort and 63 years in our cohort. Tumor stage was also equally distributed between cohorts with 18.5\%, 18.3\% and 19.2\% FIGO stage IV patients in Ferrandina's cohort, Gerestein's cohort and the validation cohort respectively. In our validation cohort $53.6 \%$ of patients underwent incomplete debulking surgery compared to 55.9\% in Ferrandina's cohort and 54.8\% in Gerestein's cohort. Apparent differences can be found in the percentage of patients with WHO-PS 0 or 1, which was 92.2\%, 74.9\% and 56.3\% in Gerestein's cohort, Ferrandina's cohort and the validation cohort respectively. Patients with a primary tumor other than ovarian cancer are only found in Ferrandina's cohort ( $N=19,9.7 \%)$.

\section{External validation}

Results of the external validation of Ferrandina's models and Gerestein's model are given in Table 3. The predictive performances of both Ferrandina models were substantially lower in our validation cohort. Differences were most prevalent for PPV or post-test probability (92.6\% compared to $68.4 \%, 69.2 \%$ and $75.0 \%$ in model A for readers 1,2 and 3 respectively) and AUC (0.81 compared to 0.56, 0.59 and 0.59 in model A for readers 1, 2 and 3 respectively). Similar changes were observed in model B where lower AUC values were noted in the validation cohort (0.82 compared to 0.55 , 0.60 and 0.59 for the different readers). However this effect was not seen when applying Gerestein's model to the validation cohort. Its predictive ability after internal validation expressed as a C-index of 0.67 in the original cohort was maintained in our cohort where we found comparable values for the AUC of 0.69, 0.61 and 0.69. 


\section{Chapter 5}

Table 2. Baseline Characteristics

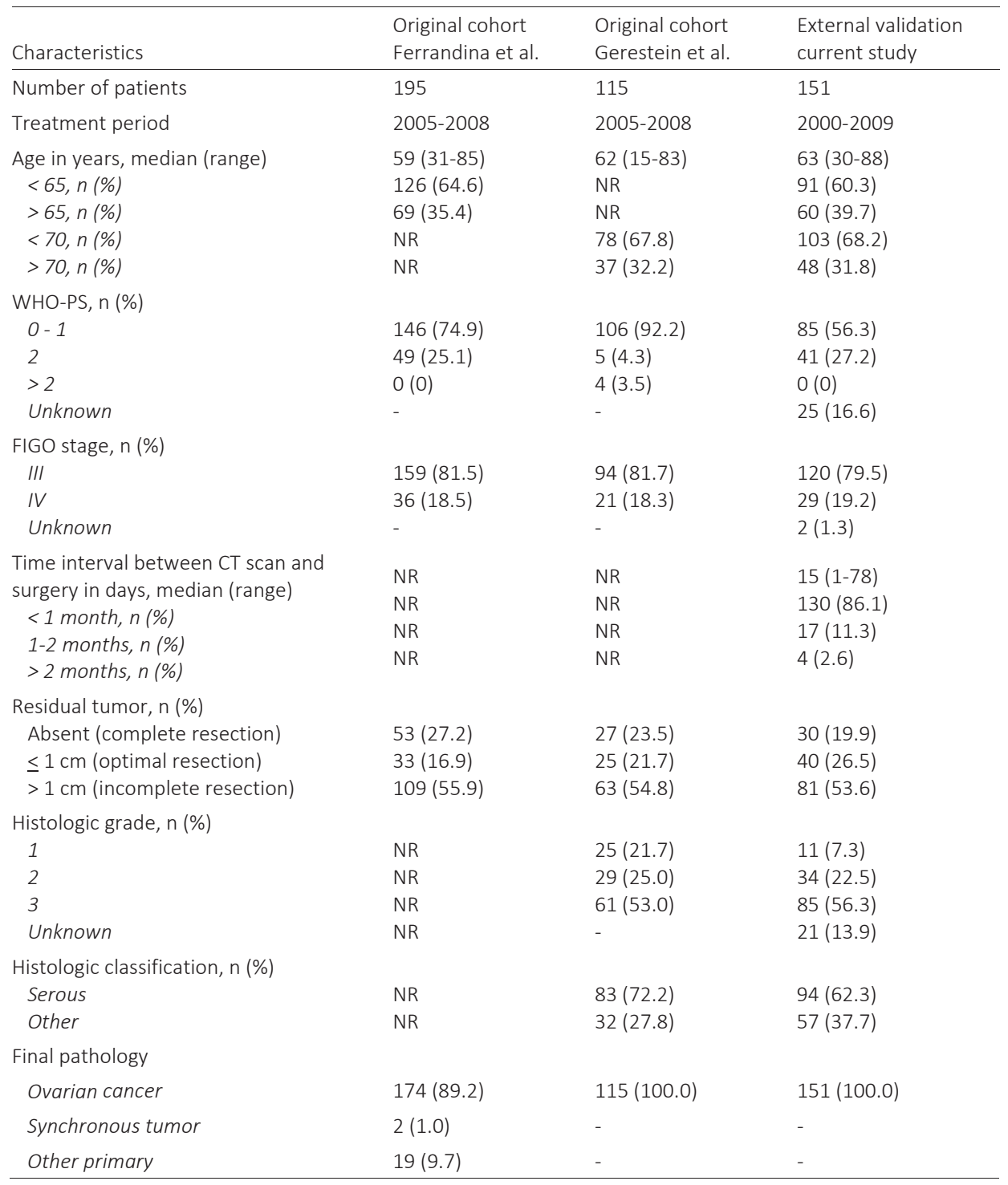

NR $=$ Not reported, WHO-PS $=$ World Health Organization Performance Status. 
Table 3. External validation of three prediction models

\begin{tabular}{|c|c|c|c|c|c|c|c|c|}
\hline \multicolumn{9}{|c|}{ 1. Ferrandina model $A$ : Cut-off $>5 / 8$} \\
\hline & Sensitivity & Specificity & PPV & NPV & $\begin{array}{l}\text { Pre-test } \\
\text { probability }\end{array}$ & $\begin{array}{l}\text { Post-test } \\
\text { probability }\end{array}$ & Improvement & $A \cup C$ \\
\hline Ferrandina et al. & 22.9 & 97.7 & 92.6 & 50.0 & 55.8 & 92.6 & +36.8 & 0.81 \\
\hline Reader 1 & 23.2 & 88.0 & 68.4 & 50.6 & 53.6 & 68.4 & +18.4 & 0.56 \\
\hline Reader 2 & 34.0 & 84.3 & 69.2 & 55.1 & 53.6 & 69.2 & +19.2 & 0.59 \\
\hline Reader 3 & 31.3 & 86.1 & 75.0 & 48.4 & 53.6 & 75.0 & +25.0 & 0.59 \\
\hline \multicolumn{9}{|c|}{ 2. Ferrandina model B: Cut-off $>3 / 5$} \\
\hline & Sensitivity & Specificity & PPV & NPV & $\begin{array}{l}\text { Pre-test } \\
\text { probability }\end{array}$ & $\begin{array}{l}\text { Post-test } \\
\text { probability }\end{array}$ & Improvement & $A \cup C$ \\
\hline Ferrandina et al. & 23.9 & 97.7 & 92.8 & 50.3 & 55.8 & 92.8 & +37.0 & 0.82 \\
\hline Reader 1 & 18.2 & 92.0 & 71.4 & 50.5 & 53.6 & 71.4 & +21.4 & 0.55 \\
\hline Reader 2 & 32.3 & 88.5 & 76.9 & 52.3 & 53.6 & 76.9 & +26.9 & 0.60 \\
\hline Reader 3 & 31.3 & 85.7 & 75.0 & 47.6 & 53.6 & 75.0 & +25.0 & 0.59 \\
\hline \multicolumn{9}{|l|}{ 3. Gerestein } \\
\hline & Sensitivity & Specificity & PPV & NPV & $\begin{array}{l}\text { Pre-test } \\
\text { probability }\end{array}$ & $\begin{array}{l}\text { Post-test } \\
\text { probability }\end{array}$ & Improvement & $\begin{array}{l}\text { C-index/ } \\
\text { AUC }\end{array}$ \\
\hline Gerestein et al. & - & - & - & - & - & - & - & 0.67 \\
\hline Reader 1 & - & - & - & - & - & - & - & 0.69 \\
\hline Reader 2 & - & - & - & - & - & - & - & 0.61 \\
\hline Reader 3 & - & - & - & - & - & - & - & 0.69 \\
\hline
\end{tabular}

$\mathrm{PPV}=$ positive predictive value, $\mathrm{NPV}=$ negative predictive value, $\mathrm{AUC}=$ area under the curve, $\mathrm{C}$-index $=$ concordance index

\section{Regression analysis of individual predictors}

The majority of predictors were significant at the level of $10 \%$ in univariable regression analysis (see Table 4). Predictors that were not significant were 'liver involvement' and 'blood platelet count' for all readers, 'bowel mesentery involvement' for reader 1 and 'peritoneal thickening and/or implants' and 'suprarenal aortic lymph nodes' for reader 3. Significant predictors were further assessed in multivariable analysis. Different combinations of predictors were found to be significant for each reader. For reader 1 , the two parameters 'peritoneal thickening and/or implants' [OR 3.806 (1.608-9.009), $p=0.002$ ] and 'omental extension' [OR 2.689 (1.137-6.362), $p=0.024$ ] were significant. For reader 2 this was the case for 'bowel mesentery involvement' [OR 3.393 (1.4897.732), $p=0.004$ ] and 'suprarenal aortic lymph nodes' [OR 2.782 (1.125-6.877), $p=0.027$ ]. Lastly, for reader 3 'diffuse peritoneal thickening' [OR 3.835 (1.297-11.340), $p=0.015$ ] and 'diaphragmatic disease [OR 2.817 (1.068-7.429), $p=0.036$ ] were significant to predict surgical outcome. 


\section{Chapter 5}

Table 4. Logistic regression analysis for prediction of incomplete debulking surgery

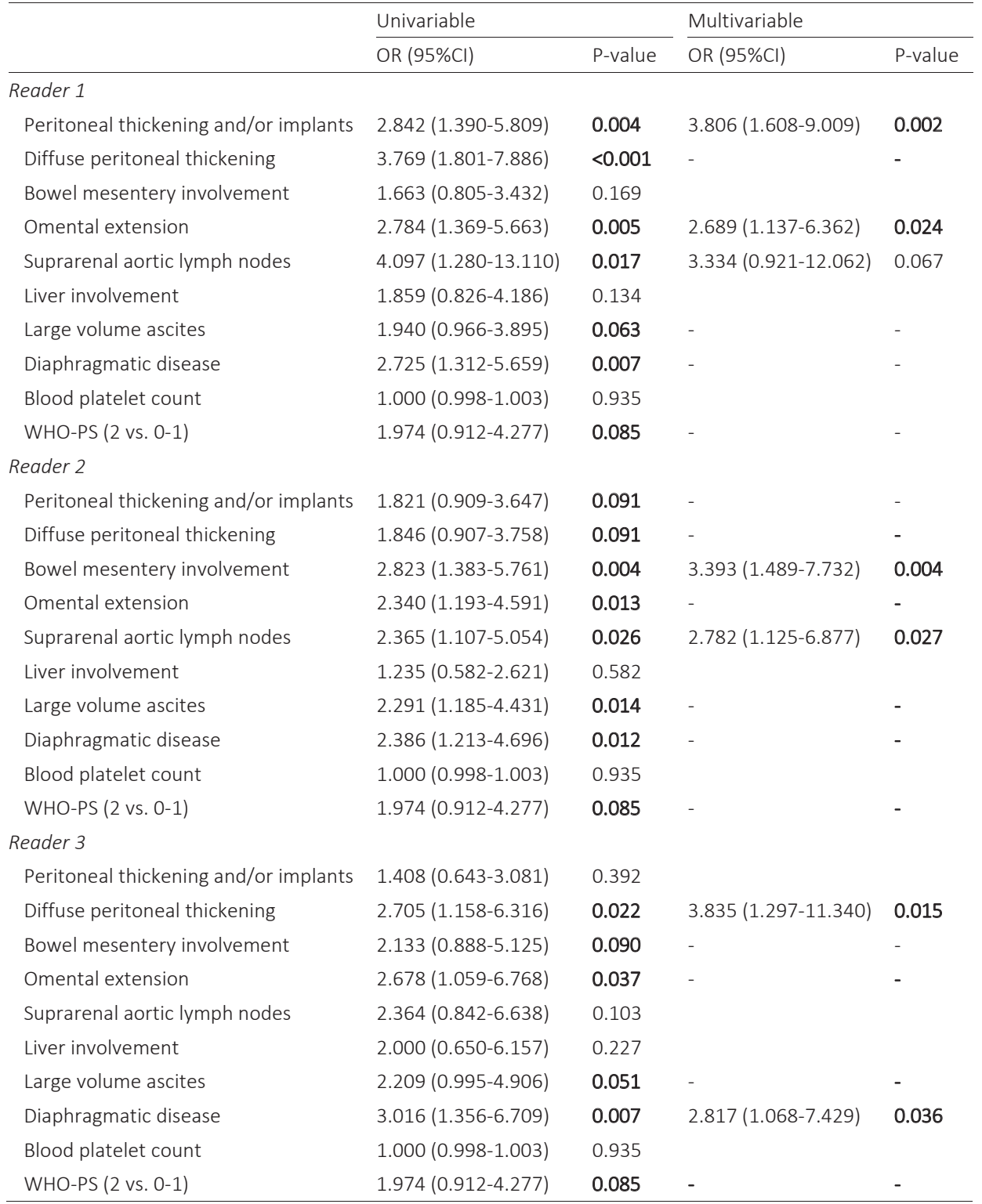

P-values marked in bold writing represent statistical significance at the level of $10 \%$ in univariable and $5 \%$ in multivariable logistic regression analysis. OR = Odds ratio, $95 \% \mathrm{Cl}=95 \%$ Confidence interval, WHO-PS $=$ World Health Organization Performance Status.

The highest interobserver agreement was found for the assessment of large volume ascites with kappa values of $0.65,0.81$ and 0.50 . For the evaluation of diffuse peritoneal thickening and omental extension only slight agreement was noted between readers. In 
the evaluation of the remaining parameters, a fair to moderate interobserver agreement was found. More results are given in Table 5.

Table 5. Interobserver agreement

\begin{tabular}{llll}
\hline & Reader 1 vs. 2 (kappa) & Reader 1 vs. 3 (kappa) & Reader 2 vs. 3 (kappa) \\
\hline Diffuse peritoneal thickening & 0.27 & 0.09 & 0.07 \\
Peritoneal implants & 0.35 & 0.27 & 0.38 \\
Bowel mesentery involvement & 0.29 & 0.19 & 0.35 \\
Omental extension & 0.46 & 0.14 & 0.17 \\
Suprarenal aortic lymph nodes & 0.47 & 0.43 & 0.48 \\
Liver disease & 0.48 & 0.43 & 0.25 \\
Large volume ascites & 0.65 & 0.81 & 0.50 \\
Diaphragmatic disease & 0.48 & 0.57 & 0.35 \\
Subjective assessment & - & 0.24 & - \\
\hline
\end{tabular}

Explanation of kappa values: $0.01-0.20=$ slight agreement, $0.21-0.40=$ fair agreement, $0.41-0.60=$ moderate agreement, $0.61-0.80=$ substantial agreement, $0.81-0.99=$ almost perfect agreement

\section{Subjective assessment}

The diagnostic value of subjective assessment is shown in Table 6. A sensitivity of $59.4 \%$ and specificity of $77.8 \%$ for reader 1 and a sensitivity of $86.4 \%$ and specificity of $40.0 \%$ for reader 3 were found. The predictive performance expressed in AUC value was 0.69 for reader 1 and 0.63 for reader 3. Data on subjective assessment were not available for reader 2 .

Table 6. Subjective assessment for prediction of incomplete debulking surgery

\begin{tabular}{llllll}
\hline & Sensitivity & Specificity & PPV & NPV & AUC \\
\hline Reader 1 & 59.4 & 77.8 & 74.5 & 63.6 & 0.69 \\
Reader 3 & 86.4 & 40.0 & 65.4 & 69.2 & 0.63 \\
\hline
\end{tabular}

PPV = positive predictive value, NPV = negative predictive value, $\mathrm{AUC}=$ area under the curve

\section{DISCUSSION}

\section{Key findings}

In this external validation study we tested the ability of three models using computed tomography to predict incomplete primary debulking surgery. We found that the predictive performances of both Ferrandina models were considerably lower when they 
were tested in our validation cohort. Gerestein's model maintained its accuracy when evaluated by three different readers and thus seems superior to be used in patients from different populations. However, none of the prediction models we validated performed well enough to be used reliably in clinical practice. In fact, the models didn't perform better than the subjective assessment of the radiologist. Numerous models have been created over the past years but surprisingly all have used different sets of parameters to predict incomplete resection. Similarly, the multivariable analysis in our study found different combinations of predictors for three different readers whilst using the same patient population.

Ideally, a model should include well-defined and reproducible parameters in order to exhibit a constant performance when used by different readers or different centers. We were able to test the reproducibility of the prediction models by asking three readers from different centres to evaluate the CT scans. We encountered a great deal of interobserver variability between our readers in the assessment of the radiographic features. This underscores the finding that the radiographic parameters are prone to some individual interpretation. This could be one of the reasons why so many different prediction models exist, why the predictive performance of the models is limited, and why external validation of a model usually results in even lower performance indices $[22 ; 26 ; 27]$. An exception to these findings was seen in the model created by Borley et al. When tested in a second cohort of 70 patients, an AUC of 0.72 was found compared to the AUC of 0.75 from the original cohort $(n=111)$ [28]. This is comparable to what we found when validating Gerestein's model; the original AUC of 0.67 was maintained in our cohort although this value is slightly lower than the AUC of 0.72 in Borley's cohort. Nevertheless, the decision to favour one model over the other when assessing a patient with advanced ovarian cancer is arbitrary and should be validated in each hospital where it is to be used.

Another explanation why models perform poorly is that they do not incorporate the surgeon's effort or skills, while surgical outcome is highly dependent on a surgeon's ability to remove all visible tumor deposits [8]. Some surgeons adopt a more aggressive approach including (partial) resection of the bowel, bladder, liver, spleen or diaphragm to achieve complete tumor resection. In consequence, there is a great spread in the percentage of complete (no residual tumor) and optimal $(<1 \mathrm{~cm}$ residual tumor) surgeries between hospitals worldwide. Optimal primary debulking rates between $35 \%$ and $92 \%$ have been reported for advanced ovarian cancer [15-24; 28-30]. Moreover, centers that have changed their strategy over time towards more radical surgery were able to achieve higher complete debulking rates [31]. When models would have been used in these hospitals, their performance would have changed over time.

The subjective assessment of the different readers seems as successful as applying predictive models. Subjective assessment had an AUC of 0.69 and 0.63 , which was comparable to the performance of Gerestein's model and even superior to the performance of Ferrandina's models in predicting incomplete surgery. However, the 
interobserver agreement for this assessment was only fair $(k=0.24)$. Presumably, the performance of the subjective assessment can be even higher when the radiologist knows the gynecological oncologist who will perform the surgery and his/her surgical skills. For example, when a gynecological oncologist never performs diaphragmatic surgery, it is easy to predict an incomplete debulking whenever diaphragmatic metastases are present on CT scan. In this study, the radiologists and gynecological oncologists did not always work together in the same hospital, and this could have influenced the current results. The lower sensitivity found for reader 1 in comparison to reader 3 in the subjective assessment could be (partly) explained by this. Because reader 1 was not familiar with the expertise of the treating physician, he often incorrectly predicted optimal surgery. This emphasizes the strength and importance of a good collaboration and tumorboard discussions between radiologists and gynecological oncologists.

\section{Limitations}

Although many more have been published, we only validated three models to predict incomplete debulking. Our decision to choose these models was based on the fact that they were created with prospective data, included only patients with advanced stage ovarian cancer and had a reasonable study size of at least 100 subjects. Moreover, other models often incorporated an abundance of predictors which complicates their clinical applicability. After conducting our analyses for this study, another prospectively validated prediction model with a good-sized population was published [30]. Unfortunately we were not able to include this study in the manuscript because our data collection was already complete and some of the variables used within the newer model were not evaluated by our readers. For future research it might be interesting to validate some of the more recently published models tested on a larger patient population [28-30].

As this study was conducted with retrospective data, selection bias and missing values are inevitable. Nonetheless we gathered an adequate sample size to conduct our analyses.

We found a relatively high percentage $(53.6 \%)$ of incomplete debulking surgery in our population of patients who were treated between 2000 and 2009. For validation purposes this caused no problems since the rates of incomplete debulking in Ferrandina's population and Gerestein's population were comparable (i.e. 55.9\% and $54.8 \%)$. However, success rates of cytoreductive surgery have improved in the last years, partly due to more extensive surgical procedures [31]. Borley et al. showed that a prediction model developed on a patient population where $53 \%$ of resections was incomplete could still significantly predict surgical outcome in a validation cohort of patients of whom 24\% underwent incomplete surgery [28]. Nevertheless, for future evaluation of more recently developed prediction models it would be more appropriate to use a population with a higher proportion of complete resections. 


\section{Chapter 5}

\section{Conclusion and Implications for practice and research}

The importance to perform complete macroscopic tumor debulking rather than optimal debulking has been consistently emphasized in recent investigations and by clinical experts. CT still remains the gold standard in preoperative ovarian cancer imaging but is limited in its ability to detect lesions smaller than $10 \mathrm{~mm}$ hence making it impossible to predict if surgery will be complete or incomplete. Newly developed and existing techniques could further aid in the predictive process. Laparoscopic staging has shown promising results in the prediction of complete primary cytoreductive surgery for women with advanced stage ovarian cancer [32]. Combined positron emission tomography/computed tomography (PET/CT) predictors have also been identified but their clinical importance is a subject of discussion [33]. Finally the value of combined positron emission tomography magnetic resonance imaging (PET/MRI) for preoperative evaluation of advanced ovarian cancer patients is upcoming in selected centres and is currently being investigated in our centre. Whether a patient will receive a complete resection will in fact largely depend on the effort and aggressiveness of the operating gynecologic oncologist. Prediction models were created to predict incomplete surgery but their predictive ability and reproducibility are questionable. An abundance of different models have been developed but few have been validated. Reported predictive performances are mediocre at most and the majority of models were not able to maintain their accuracy at external validation as was proven in this manuscript. In conclusion, the present prediction models are not reliable enough to be used in clinical decision-making. Yet the decision whether a patient will benefit more from primary surgery or from neoadjuvant chemotherapy in the event that incomplete surgery is expected is clinically very relevant. However this decision should be based more on the experience of and collaboration within the multidisciplinary oncologic team rather than on the outcome of a prediction model. 


\section{REFERENCES}

1 De Angelis R, Sant M, Coleman MP et al (2014) Cancer survival in Europe 1999-2007 by country and age: results of EUROCARE--5-a population-based study. Lancet Oncol 15:23-34

2 Surveillance E, and End Results (SEER) Program SEER 9 Incidence \& U.S. Mortality Research Data (19732011). In: National Cancer Institute D, Surveillance Research Program, Surveillance Systems Branch, released April 2014, based on the November 2013 submission, (ed),

3 Vergote I, Trope CG, Amant F et al (2010) Neoadjuvant chemotherapy or primary surgery in stage IIIC or IV ovarian cancer. N Engl J Med 363:943-953

4 du Bois A, Reuss A, Pujade-Lauraine E, Harter P, Ray-Coquard I, Pfisterer J (2009) Role of surgical outcome as prognostic factor in advanced epithelial ovarian cancer: a combined exploratory analysis of 3 prospectively randomized phase 3 multicenter trials: by the Arbeitsgemeinschaft Gynaekologische Onkologie Studiengruppe Ovarialkarzinom (AGO-OVAR) and the Groupe d'Investigateurs Nationaux Pour les Etudes des Cancers de I'Ovaire (GINECO). Cancer 115:1234-1244

5 Chi DS, Eisenhauer EL, Lang J et al (2006) What is the optimal goal of primary cytoreductive surgery for bulky stage IIIC epithelial ovarian carcinoma (EOC)? Gynecol Oncol 103:559-564

6 Griffiths CT (1975) Surgical resection of tumor bulk in the primary treatment of ovarian carcinoma. Natl Cancer Inst Monogr 42:101-104

7 Kehoe S, Hook J, Nankivell M et al (2015) Primary chemotherapy versus primary surgery for newly diagnosed advanced ovarian cancer (CHORUS): an open-label, randomised, controlled, non-inferiority trial. Lancet 386:249-257

8 Aletti GD, Gostout BS, Podratz KC, Cliby WA (2006) Ovarian cancer surgical resectability: relative impact of disease, patient status, and surgeon. Gynecol Oncol 100:33-37

9 Forstner R, Hricak H, White S (1995) CT and MRI of ovarian cancer. Abdom Imaging 20:2-8

10 Meyer JI, Kennedy AW, Friedman R, Ayoub A, Zepp RC (1995) Ovarian carcinoma: value of CT in predicting success of debulking surgery. AJR Am J Roentgenol 165:875-878

11 Nelson BE, Rosenfield AT, Schwartz PE (1993) Preoperative abdominopelvic computed tomographic prediction of optimal cytoreduction in epithelial ovarian carcinoma. J Clin Oncol 11:166-172

12 Qayyum A, Coakley FV, Westphalen AC, Hricak H, Okuno WT, Powell B (2005) Role of CT and MR imaging in predicting optimal cytoreduction of newly diagnosed primary epithelial ovarian cancer. Gynecol Oncol 96:301-306

13 Byrom J, Widjaja E, Redman CW, Jones PW, Tebby S (2002) Can pre-operative computed tomography predict resectability of ovarian carcinoma at primary laparotomy? BJOG 109:369-375

14 Kebapci M, Akca AK, Yalcin OT, Ozalp SS, Calisir C, Mutlu F (2010) Prediction of suboptimal cytoreduction of epithelial ovarian carcinoma by preoperative computed tomography. Eur J Gynaecol Oncol 31:44-49

15 Bristow RE, Duska LR, Lambrou NC et al (2000) A model for predicting surgical outcome in patients with advanced ovarian carcinoma using computed tomography. Cancer 89:1532-1540

16 Dowdy SC, Mullany SA, Brandt KR, Huppert BJ, Cliby WA (2004) The utility of computed tomography scans in predicting suboptimal cytoreductive surgery in women with advanced ovarian carcinoma. Cancer 101:346-352

17 Ferrandina G, Sallustio G, Fagotti A et al (2009) Role of CT scan-based and clinical evaluation in the preoperative prediction of optimal cytoreduction in advanced ovarian cancer: a prospective trial. $\mathrm{Br} J$ Cancer 101:1066-1073

18 Gerestein CG, Eijkemans MJ, Bakker J et al (2011) Nomogram for suboptimal cytoreduction at primary surgery for advanced stage ovarian cancer. Anticancer Res 31:4043-4049

19 Jung DC, Kang S, Kim MJ, Park SY, Kim HB (2010) Multidetector CT predictors of incomplete resection in primary cytoreduction of patients with advanced ovarian cancer. Eur Radiol 20:100-107

20 Jung DC, Kang S, Kim SC et al (2013) Use of complex surgical procedures, patterns of tumor spread, and CA-125 predicts a risk of incomplete cytoreduction: a Korean Gynecologic Oncology Group study (KGOG3022). Gynecol Oncol 131:336-340 


\section{Chapter 5}

21 Kim HJ, Choi CH, Lee YY et al (2014) Surgical outcome prediction in patients with advanced ovarian cancer using computed tomography scans and intraoperative findings. Taiwan J Obstet Gynecol 53:343347

22 Mackintosh ML, Rahim R, Rajashanker B et al (2014) CT scan does not predict optimal debulking in stage III-IV epithelial ovarian cancer: a multicentre validation study. J Obstet Gynaecol 34:424-428

23 Mousavi AS, Mazhari MM, Guilani MM, Ghaemmaghami F, Behtash N, Akhavan S (2010) Can primary optimal cytoreduction be predicted in advanced epithelial ovarian cancer preoperatively? World J Surg Oncol 8:11

24 Stashwick C, Post MD, Arruda JS et al (2011) Surgical risk score predicts suboptimal debulking or a major perioperative complication in patients with advanced epithelial ovarian, fallopian tube, or primary peritoneal cancer. Int J Gynecol Cancer 21:1422-1427

25 Rutten MJ, van de Vrie R, Bruining A et al (2015) Predicting surgical outcome in patients with International Federation of Gynecology and Obstetrics stage III or IV ovarian cancer using computed tomography: a systematic review of prediction models. Int J Gynecol Cancer 25:407-415

26 Axtell AE, Lee MH, Bristow RE et al (2007) Multi-institutional reciprocal validation study of computed tomography predictors of suboptimal primary cytoreduction in patients with advanced ovarian cancer. J Clin Oncol 25:384-389

27 Gemer O, Gdalevich M, Ravid M et al (2009) A multicenter validation of computerized tomography models as predictors of non- optimal primary cytoreduction of advanced epithelial ovarian cancer. Eur J Surg Oncol 35:1109-1112

28 Borley J, Wilhelm-Benartzi C, Yazbek J et al (2015) Radiological predictors of cytoreductive outcomes in patients with advanced ovarian cancer. BJOG 122:843-849

29 Janco JM, Glaser G, Kim B et al (2015) Development of a prediction model for residual disease in newly diagnosed advanced ovarian cancer. Gynecol Oncol 138:70-77

30 Suidan RS, Ramirez PT, Sarasohn DM et al (2014) A multicenter prospective trial evaluating the ability of preoperative computed tomography scan and serum CA-125 to predict suboptimal cytoreduction at primary debulking surgery for advanced ovarian, fallopian tube, and peritoneal cancer. Gynecol Oncol 134:455-461

31 Wahner Hendrickson AE, Hawthorne KM, Goode EL et al (2015) Assessment of published models and prognostic variables in epithelial ovarian cancer at Mayo Clinic. Gynecol Oncol 137:77-85

32 Petrillo M, Vizzielli G, Fanfani F et al (2015) Definition of a dynamic laparoscopic model for the prediction of incomplete cytoreduction in advanced epithelial ovarian cancer: Proof of a concept. Gynecol Oncol. 10.1016/j.ygyno.2015.07.095

33 Risum S, Hogdall C, Loft A et al (2008) Prediction of suboptimal primary cytoreduction in primary ovarian cancer with combined positron emission tomography/computed tomography--a prospective study. Gynecol Oncol 108:265-270 



\section{Chapter 6}

\section{ABSTRACT}

Objectives: Selecting patients with ovarian cancer who will benefit from debulking surgery is pivotal to optimize survival. The limited accuracy of preoperative computed tomography (CT) stresses the need for alternative imaging techniques. Our objective was to investigate the diagnostic performance of integrated positron emission tomography magnetic resonance imaging (PET/MRI) in the assessment of ovarian cancer spread in patients undergoing debulking surgery.

Methods: In this prospective pilot study ten patients with advanced ovarian cancer underwent contrast-enhanced PET/MRI scan with additional diffusion weighted magnetic resonance imaging (DW-MRI). The diagnostic performance of PET/MRI was compared to contrast-enhanced CT for detection of tumour sites in the peritoneal cavity. Secondly the value of DW-MRI compared to PET/MRI was investigated.

Results: CT showed a sensitivity of $60 \%$ and specificity of $79 \%$ for the detection of peritoneal tumour sites compared to $71 \%$ and $79 \%$ for PET/MRI respectively. DW-MRI showed a sensitivity and specificity of $70 \%$ and $78 \%$.

Conclusions: PET/MRI and DW-MRI are feasible for preoperative peritoneal staging of ovarian cancer and showed a trend towards a superior sensitivity in comparison to CT. The results for PET/MRI are mainly a reflection of the contribution of MRI; PET imaging was not able to reliably predict tumour depositions. 


\section{INTRODUCTION}

Epithelial ovarian cancer is the most lethal of all gynaecological malignancies with a 5year survival in the Netherlands of $35 \%$ [1]. It is often diagnosed in an advanced stage due to its aggressive nature and paucity of symptoms. Metastatic pathways include intraperitoneal, lymphogenous and haematogenous spread. Initial choice of treatment for patients with advanced stage ovarian cancer is primary debulking surgery (PDS). The most important predictor of survival in ovarian cancer is complete tumour resection with no sign of residual disease [2; 3]. If preoperative assessment indicates that optimal or complete debulking - defined as $<1 \mathrm{~cm}$ or no residual tumour - is not feasible, patients can be assigned to neo-adjuvant chemotherapy with the option of interval debulking surgery (IDS) after a second assessment [3; 4]. Incomplete resection has little beneficial effects for survival while it can cause substantial perioperative morbidity. Choosing the right treatment strategy by selecting patients who will benefit from primary surgery is therefore pivotal to optimize ovarian cancer survival.

Computed tomography (CT) is currently considered gold standard for preoperative staging but is limited in its ability to detect lesions smaller than $10 \mathrm{~mm}$ hence making it difficult to predict whether surgery will be complete [5]. Incomplete PDS rates between $8 \%$ and $65 \%$ have been reported [6-19]. This has led to the exploration of other imaging techniques such as magnetic resonance imaging (MRI) and positron emission tomography (PET) to evaluate ovarian cancer. MRI provides anatomic detail in staging of local tumour extent due to its high soft tissue resolution. Diffusion weighted imaging (DWI) is a functional MRI technique which uses the diffusion process of water molecules in biological tissues to supplement conventional MRI [20]. Studies in ovarian and other gynaecological cancers have shown that diffusion-weighted magnetic resonance imaging (DW-MRI) is able to detect peritoneal dissemination and lymphadenopathy with high accuracy [20-22]. PET imaging provides a different functional technique and is generally used in combination with $\mathrm{CT}$ to detect metabolically active tumour sites and lymph nodes. PET-CT has limited use in ovarian cancer staging due to frequent false negative results for detecting small peritoneal metastases [23; 24].

More recently, efforts were made to integrate PET and MRI in a combined wholebody positron emission tomography magnetic resonance imaging (PET/MRI) scan [25; 26]. This method integrates morphologic and metabolic features within a single examination. Optionally, PET/MRI can be supplemented with DWI. A study assessing the diagnostic benefit of DWI in an PET/MRI protocol in patients with various pelvic malignancies found high diagnostic performance for the detection of malignant lesions when using PET/MRI both with and without DWI [27]. The most appropriate clinical applications in which PET/MRI can be useful still have to be established. We hypothesize that PET/MRI is a feasible technique for preoperative staging of ovarian cancer. The primary objective of this prospective study is to compare the diagnostic performance of 


\section{Chapter 6}

PET/MRI with CT in the assessment of ovarian cancer spread. Our secondary objective is to assess the diagnostic value of DW-MRI in comparison to PET/MRI in this matter.

\section{MATERIALS AND METHODS}

This study was approved by the Maastricht University Medical Centre Review Board and has been conducted according to the principles of the Declaration of Helsinki (Fortaleza, October 2013). The STARD-2015 guidelines for diagnostic accuracy studies were used [28].

\section{Study design and participants}

This was a prospective pilot study including women with clinical FIGO (International Federation of Gynecologic Oncology) stage IIB-IV epithelial ovarian carcinoma undergoing debulking surgery. Ten patients were selected to undergo PDS or IDS after consultation of a multidisciplinary team, as is standard clinical practice in our hospital. Inoperable patients or patients in whom surgery was deemed not feasible were not included in the study. No adjustments to the surgical procedure were made with regard to this study. Written informed consent was obtained from all participants. Underaged, incapacitated, pregnant and lactating subjects were excluded from participation. Patients ineligible to undergo PET/MRI examination due to the presence of non-MR compatible implants or claustrophobia were also excluded.

\section{Imaging procedure}

PET/MRI scanning was performed with the Biograph mMR system (Siemens Healthcare, Erlangen, Germany). This system integrates a 3Tesla MRI and PET camera, making it possible to acquire simultaneous whole-body MRI and PET images. Imaging was performed with a region of interest between the skull base and proximal femur. The radiotracer $18 \mathrm{~F}$-labeled fluorodeoxyglucose (18F-FDG) was used for PET imaging. A dose of $2 \mathrm{MBq} / \mathrm{kg}$ 18F-FDG was administered to the patient. During the PET acquisition, the following MRI sequences were concomitantly obtained: T2-weighted halffourier acquisition single-shot turbo spin echo images in axial and coronal planes, axial DWI with a b-value of $1000 \mathrm{~s} / \mathrm{mm}^{2}$, and axial and coronal 3DT1-weighted gradient echo volumetric interpolated breath-hold examination images before and after intravenous administration of gadolinium-based contrast (Gadovist). Post-processing methods were used for fusion of the contrast-enhanced series with the PET images and the axial T2weighted series with the DWI images. Total PET/MRI scanning time was approximately 90 minutes. A comprehensive overview of the MRI protocol is provided in Table 1. 
Table 1. MRI acquisition parameters in the PET/MRI protocol

\begin{tabular}{|c|c|c|c|c|c|c|c|}
\hline Parameter & $\begin{array}{l}\text { Axial T2w } \\
\text { HASTE }\end{array}$ & $\begin{array}{l}\text { Coronal T2w } \\
\text { HASTE }\end{array}$ & Axial DWI & $\begin{array}{l}\text { Axial T1w } \\
\text { VIBE }\end{array}$ & $\begin{array}{l}\text { Coronal T1w } \\
\text { VIBE }\end{array}$ & $\begin{array}{l}\text { Axial ceT1w } \\
\text { VIBE }\end{array}$ & $\begin{array}{l}\text { Coronal } \\
\text { ceT1w VIBE }\end{array}$ \\
\hline Voxel size $(\mathrm{mm})$ & $1.7 \times 1.4 \times 5.5$ & $1.6 \times 1.6 \times 5.0$ & $2.9 \times 2.7 \times 6.0$ & $1.7 \times 1.2 \times 3.0$ & $1.5 \times 1.1 \times 3.0$ & $1.7 \times 1.2 \times 3.0$ & $1.5 \times 1.1 \times 3.0$ \\
\hline Slice thickness (mm) & 5.5 & 5.0 & 6.0 & 3.0 & 3.0 & 3.0 & 3.0 \\
\hline Field of view (mm) & 450 & 400 & 400 & 380 & 360 & 380 & 360 \\
\hline Number of stacks & 3 & 3 & 3 & 3 & 4 & 3 & 4 \\
\hline Repetition time (ms) & 1600 & 1400 & 10400 & 5.44 & 3.30 & 5.44 & 3.30 \\
\hline Echo time 1 (ms) & 113 & 96 & 77 & 2.47 & 1.2 & 2.47 & 1.2 \\
\hline Echo time 2 (ms) & / & / & / & 3.80 & / & 3.80 & / \\
\hline Flip angle $\left({ }^{\circ}\right)$ & 120 & 143 & / & 9 & 9 & 9 & 9 \\
\hline Fat suppression & None & None & SPAIR & None & None & Q-fat sat. & Q-fat sat. \\
\hline Base resolution & 320 & 256 & 150 & 320 & 320 & 320 & 320 \\
\hline Bandwidth ( $\mathrm{Hz} / \mathrm{Px})$ & 710 & 710 & 2084 & 980 & 600 & 980 & 600 \\
\hline B-value (s/mm2) & / & / & 1000 & / & / & / & / \\
\hline Directions & / & / & 3 & / & / & / & / \\
\hline Contrast agent & None & None & None & None & None & Gadolinium & Gadolinium \\
\hline
\end{tabular}

MRI, magnetic resonance imaging; PET/MRI, positron emission tomography magnetic resonance imaging; $T 2 \mathrm{~W}$ HASTE, T2-weighted half-fourier acquisition single-shot turbo spin echo; DWI, diffusion-weighted imaging; T1W VIBE, T1-weighted volumetric interpolated breath-hold examination; ce, contrast-enhanced; SPAIR, spectral attenuated inversion recovery; Q-fat sat, quick fat saturation.

\section{Analysis}

Preoperative contrast-enhanced CT scans of the abdominal and thoracic regions were available from clinical diagnostic work-up. CT scans were re-evaluated for this study by a radiologist with expertise in gynaecological MRI without access to previous CT reports and PET/MRI images or reports. The PET/MRI scans were analyzed in two consecutive steps. During the first step the MRI images and the PET images were examined separately by the same radiologist and a nuclear medicine specialist. During the second step, the two readers matched their findings in a consensus meeting. Readers were blinded for the images and reports of CT examinations. The readers were at all times unaware of clinical information (with exception to the fact that advanced ovarian cancer was suspected) and reference standard results. The results for PET/MRI reflect the findings of PET/MRI without DWI sequences. However, as a secondary objective DWMRI images were analyzed and compared to PET/MRI images. This secondary analysis was performed after matching of the PET/MRI images of the radiologist and the nuclear medicine specialist. As a consequence, analysis of DW-MRI was blinded to CT, but not blinded to PET/MRI.

A series of anatomical regions were scored for the absence or presence of tumour on a standardized report file for CT, PET/MRI and DW-MRI. The presence and location of distant metastases and pelvic, para-aortic and/or supradiaphragmatic lymphadenopathy was also recorded. Surgical staging combined with histopathologic examination was used 
as reference standard. During surgery all regions were systematically explored and the presence or absence of metastases was recorded. Systematic lymphadenopathy was not part of the standard surgical procedure. Only enlarged or suspicious lymph nodes were removed. The pathologist examined the resected tissue according standard practice. The surgeon and pathologist were aware of patient data and CT findings but blinded to $\mathrm{PET} / \mathrm{MRI}$ findings.

Since this was a small pilot study, the sample size was expected to be insufficient to show statistical differences between $\mathrm{CT}$ and PET/MRI. The data were therefore presented in an exploratory data analysis. True and false positive and negatives were recorded for each region for $\mathrm{CT}, \mathrm{PET} / \mathrm{MRI}$ and DW-MRI from which sensitivity, specificity, positive predictive value, negative predictive value and accuracy were calculated.

\section{RESULTS}

\section{Study population}

Ten patients were included between April 2015 and April 2016 in the Maastricht University Medical Centre (Table 2). Six patients underwent PDS and four patients underwent IDS after three cycles of neoadjuvant chemotherapy. Mean patient age was 64.1 years. Six patients were clinically staged as FIGO stage III ovarian cancer and four patients as FIGO stage IV. After surgery, one patient with clinical FIGO stage IIIC turned out to have a FIGO stage IC adult granulosa cell tumour (Figure 1). In one patient we found a histopathologic diagnosis of a female adnexal tumour of probable Wolffian origin (FATWO, Figure 2). A FATWO is a rare ovarian tumour and generally considered as a low grade tumour but metastatic and/or recurrent disease has been described [29]. Mean time period between CT and PET/MRI was 18 days (range 9-36 days). Surgery was performed within 7 days after PET/MRI. No adverse events occurred during the conduct of this study.

\section{Diagnostic performance for detection of intraperitoneal metastases}

Table 2 shows the diagnostic performances of CT, PET/MRI and DW-MRI in a per-patient analysis. FDG accumulation was not substantial enough in any of the patients to contribute to a certain diagnosis with the exception of mild FDG accumulation in the diaphragm in two patients and paracardial accumulation in the suspicion of lymphadenopathy in three patients. Therefore, the results of PET/MRI rely mainly on the examination of the MRI images. There were no clear differences in diagnostic performance when comparing patients with PDS and IDS. 


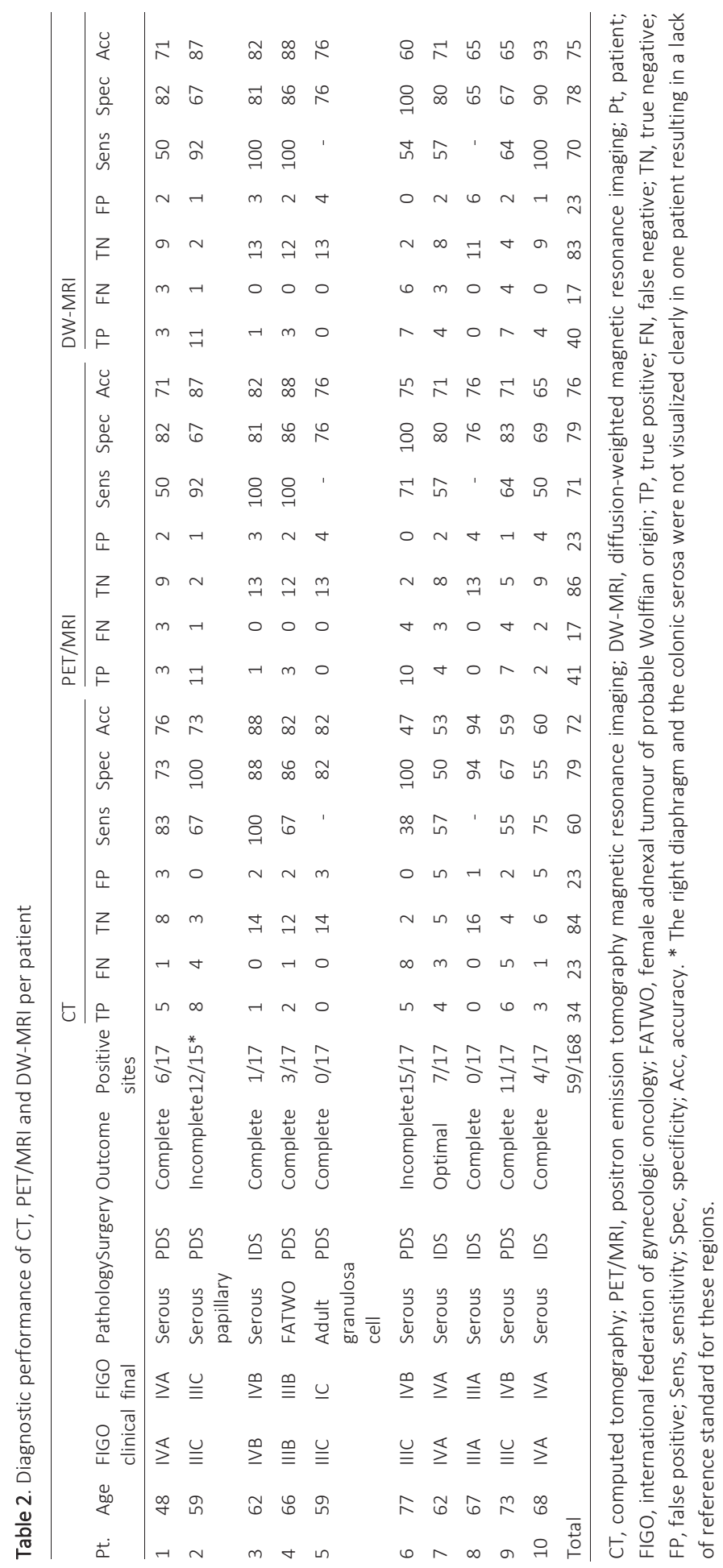




\section{Chapter 6}

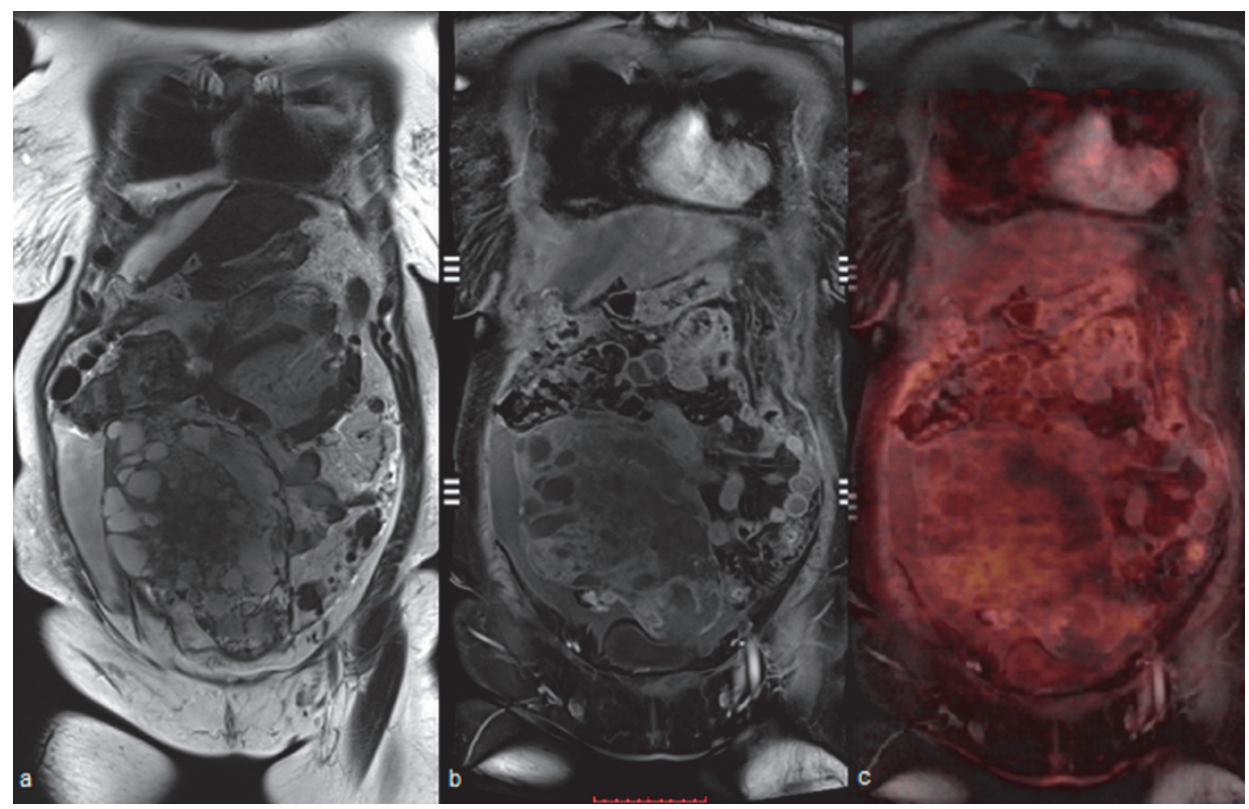

Figure 1. Example of coronal PET/MRI sequences in a patient with an adult granulosa cell tumour

a. T2-weighted MRI, b. Contrast-enhanced T1-weighted MRI, c. Fused contrast-enhanced T1-weighted PET/MRI. In this 59-year old patient PET/MRI imaging showed a large tumour in the right abdomen, histological diagnosis proved an adult granulosa cell tumour. PET/MRI, positron emission tomography magnetic resonance imaging; MRI, magnetic resonance imaging.

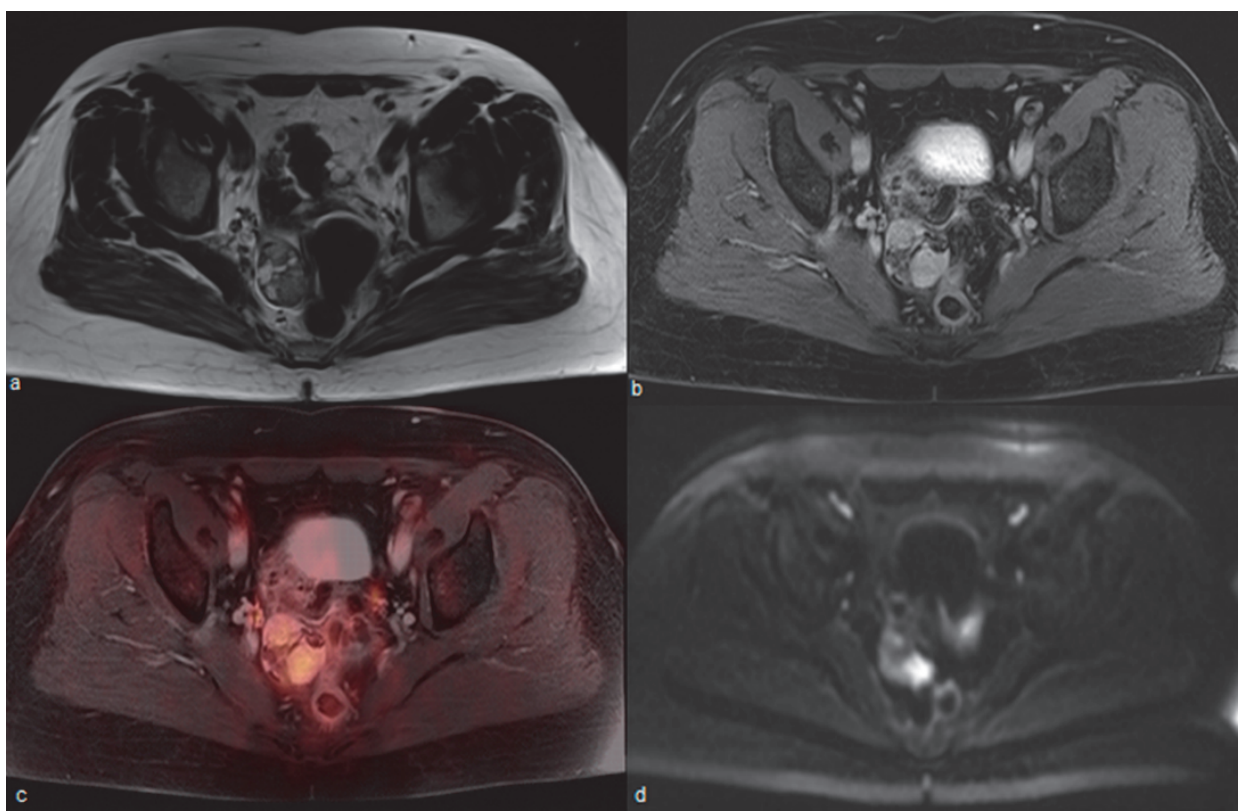


Figure 2. Example of axial PET/MRI and DWI sequences in a patient with a FATWO

a. T2-weighted MRI, b. Contrast-enhanced T1-weighted MRI, c. Fused contrast-enhanced T1-weighted PET/MRI, d. DWI. 66-year old patient with a right ovarian tumour (a,b) that shows accumulation on PET (c) and diffusion restriction on DWI (d). Histologic diagnosis was a FATWO. PET/MRI, positron emission tomography magnetic resonance imaging; DWI, diffusion-weighted imaging; MRI, magnetic resonance imaging; FATWO, female adnexal tumour of probable Wolffian origin.

Table 3 shows the diagnostic performance of the different imaging techniques per region. When assessing specific tumour regions, high sensitivity rates were found for $\mathrm{PET} / \mathrm{MRI}$ and DW-MRI in comparison with CT in the assessment of pelvic sites (regions 1-4). To the contrary, low sensitivity rates were reported for all three techniques in the evaluation of metastases to the small bowel and colon. Figure 3 shows an example of the imaging findings in a patient with diaphragmatic implants. Only one patient had a proven lymph node metastasis situated in the external iliac region at pathologic review, which was detected on CT and PET/MRI, but missed on DWI. Paracardial enlarged lymph nodes were suspected on DW-MRI and PET/MRI in three patients and on CT in one patient but histologic proof was not retrieved. Port-site metastases were found in two patients who had both undergone diagnostic laparoscopy after preoperative CT and before PET/MRI examination. DW-MRI and PET/MRI both identified the port-site metastases in subject 1 but missed the metastases in subject 2. No distant metastases were found in the remainder of patients.

The overall diagnostic performance of CT, PET/MRI and DW-MRI in the detection of intraperitoneal metastases is summarized in Table 4. PET/MRI and DW-MRI showed a non-inferiority to CT and possibly a superior sensitivity (sensitivity of $71 \%$ for PET/MRI and $70 \%$ for DW-MRI) in comparison to CT (sensitivity of $60 \%$ ). The significance of these differences could not be substantiated statistically due to the small sample size. No relevant differences were found between PET/MRI and DW-MRI. 


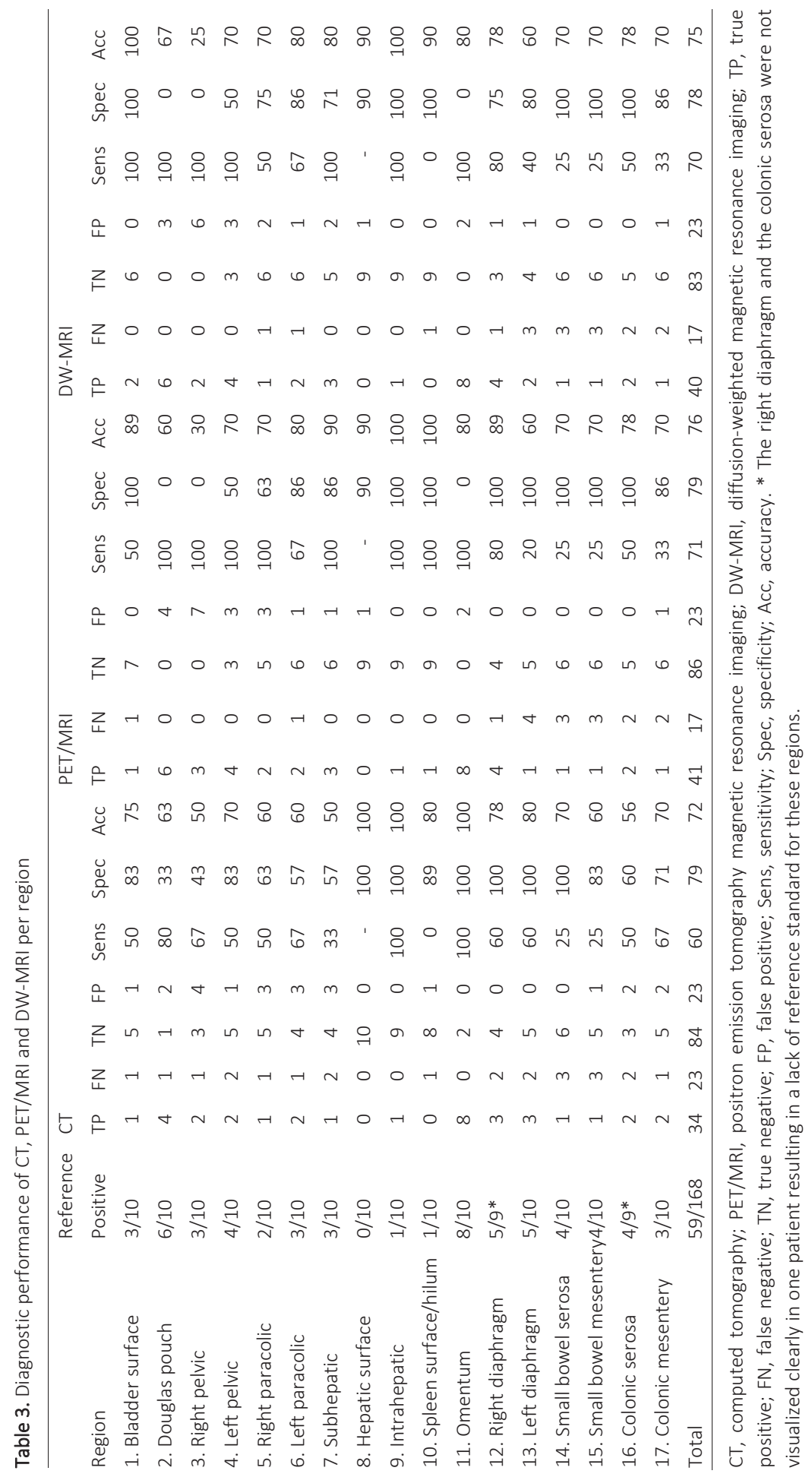




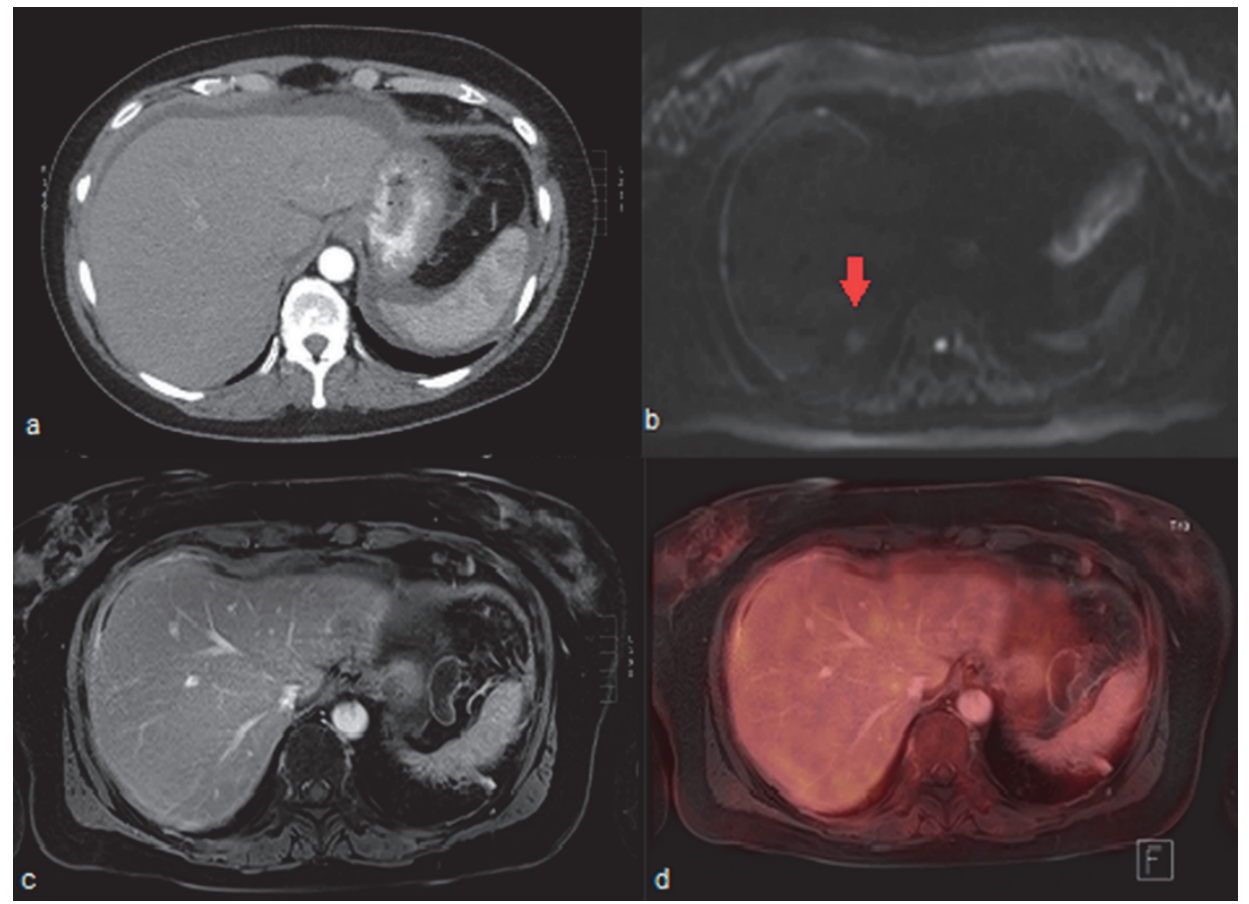

Figure 3. Example of axial CT, PET/MRI and DWI sequences in a patient with diaphragmatic implants

a. Contrast-enhanced CT, b. DWI, c. Contrast-enhanced T1-weighted MRI, d. Fused contrast-enhanced T1weighted PET/MRI. In this subject a tumour deposit in the right diaphragm (red arrow) was visualized on DWI (b) while it was missed on CT (a), MRI (c) and on fused PET/MRI (d). CT, computed tomography; PET/MRI, positron emission tomography magnetic resonance imaging; DW-MRl, diffusion-weighted magnetic resonance imaging; MRI, magnetic resonance imaging

Table 4. Diagnostic performance of CT, PET/MRI and DW-MRI in detection of intraperitoneal ovarian cancer metastases

\begin{tabular}{llll}
\hline & CT & PET/MRI & DW-MRI \\
\hline Sensitivity & 60 & 71 & 70 \\
Specificity & 79 & 79 & 78 \\
Positive predictive value & 60 & 64 & 63 \\
Negative predictive value & 79 & 83 & 83 \\
Accuracy & 72 & 76 & 75 \\
\hline
\end{tabular}

$\mathrm{CT}$, computed tomography; PET/MRI, positron emission tomography magnetic resonance imaging; DW-MRI, diffusion-weighted magnetic resonance imaging; 


\section{DISCUSSION}

We have confirmed our hypothesis that PET/MRI is a feasible technique that can be used as an alternative to CT in intraperitoneal preoperative staging of patients with ovarian cancer. In this feasibility study, an increase of approximately $10 \%$ in sensitivity could be reached with a comparable specificity. Similar results were found with DWMRI. Due to the limited number of patients, this increase in sensitivity in comparison with CT could not be proven statistically.

The diagnostic performance of PET/MRI was determined by MRI rather than by PET. PET imaging was hardly able to detect any intraperitoneal tumour sites. In some instances, PET images were only rendered positive after consensus review of the PET and MRI images when subtle FDG accumulation was noticed in certain tumour sites scored positive on MRI. It is known that PET has limited sensitivity for the detection of small metastases as can be present in peritoneal carcinomatosis due to ovarian cancer $[23 ; 24 ; 30]$. It is therefore plausible that the tumour sites in our study subjects were too small to be able to generate a metabolic signal on PET imaging.

The diagnostic accuracy of DW-MRI and PET/MRI seems to be comparable. Only a minority of tumour sites were predicted differently between both imaging techniques. Even though there were no clear differences in diagnostic accuracy between PET/MRI and DW-MRI, there are certain advantages of DW-MRI over PET/MRI. Scan time for DW$M R I$ is considerably shorter than for PET/MRI which causes less patient discomfort and reduces costs. Secondly, integrated PET/MRI is only available in a handful of specialized centres around the world and radiologists are not yet familiar with the sequences provided by PET/MRI. DW-MRI is also not routinely used for ovarian cancer. Nevertheless, radiologists have more experience with DW-MRI in other cancers, making it easier to adapt this technique in ovarian cancer. The radiologist also subjectively experienced higher levels of confidence in assessing tumour sites with the addition of DW-MRI.

The results for PET/MRI and DW-MRI suggest a superior sensitivity for the assessment of peritoneal implants in comparison to CT. Due to the small sample size this could not be confirmed through statistical analysis. The possible difference in sensitivity can have clinical impact as it might prevent surgical intervention in case of extensive superficial tumour spread. Two subjects in this study underwent incomplete PDS due to very extensive peritoneal carcinomatosis. In both these subjects, PET/MRI and DW-MRI showed a higher sensitivity in comparison to CT; in a clinical setting this would have possibly guided further management decisions towards chemotherapy instead of surgical intervention. Nowadays, diagnostic laparoscopy is often used to detect and assess peritoneal carcinomatosis in addition to CT [31; 32]. However, it is restricted to specific intraperitoneal sites. Retroperitoneal lymph nodes cannot be inspected and due to its invasive nature it requires hospitalisation and can cause perioperative and postoperative morbidity [33]. Imaging techniques, such as PET/MRI or 
DW-MRI could potentially replace diagnostic laparoscopy with the advantage that they can assess retroperitoneal regions without the disadvantage of an increased morbidity.

The sensitivity of $70 \%$, specificity of $78 \%$ and accuracy of $75 \%$ we established for DW-MRI in this study are inferior to the reported diagnostic performance for DW-MRI in peer literature. Fuji et al retrospectively studied gynaecological cancer patients and predicted the presence or absence of peritoneal dissemination of at least $5 \mathrm{~mm}$ with a sensitivity of $90 \%$ and a specificity of $96 \%$ for DW-MRI. However, the results were based on a yes-no decision and the tumour presence was not specified according to region, which explains the high performance [21]. In contrast, Michielsen et al. subjected 32 ovarian cancer patients to CT, DW-MRI and PET-CT in a prospective study and evaluated the absence or presence of tumour in specified regions; DW-MRI showed an accuracy of 91\% for detection of intraperitoneal metastases in comparison to $75 \%$ for CT [20]. The lower diagnostic performance for DW-MRI that we found in our study can be explained by the limited experience with PET/MRI and the related scan protocol. Clinical applications are being explored and consequently ready-to-use protocols are not yet available. The scan protocol was specifically designed for this study and had only been tested on healthy volunteers before the start of this study. During the course of this study the scan protocol was further optimized to deal with difficulties arising when patients had pleural effusion or large quantities of ascites which hinders respiratory triggering and impedes acquisition of diagnostic quality images. Respiratory triggering was substituted by breathold acquisition techniques with the disadvantage that many of the patients were not able to execute the relatively long breathhold commands. This resulted in breathing and movement artefacts in both DWI as well as contrast-enhanced sequences in almost all patients. Two patients had undergone bilateral total hip replacement which caused substantial artefacts and hindered assessment of pelvic regions. Further optimization of imaging protocols is expected to contribute to an improved diagnostic performance.

There are several limitations to this study. The most important limitation is the small sample size which was insufficient to prove statistical significance in the differences between imaging techniques. However, the main reason to perform this study was to investigate the feasibility of PET/MRI in comparison to CT in the assessment of peritoneal ovarian tumour metastases. The information found in this study can be used to generate new hypotheses and to perform power calculations for future studies. The small sample size also prevents us from making any confident assumptions regarding the assessment of lymphadenopathies by PET/MRI and DW-MRI. Other authors found that DW-MRI was able to detect pathologic lymph nodes with a sensitivity of $83 \%$ in comparison with $25 \%$ for conventional MRI in uterine and cervical cancer [22] and an accuracy of $87 \%$ in comparison to $71 \%$ for CT in ovarian cancer [20]. Another limitation was the limited experience with PET/MRI before the start of the study. Plausibly this study was influenced by a learning curve. An increase of experience due to adequate training and higher exposure can influence the subsequent diagnostic performance of 


\section{Chapter 6}

PET/MRI. Moreover, increased experience can also lead to improved imaging protocols and standardisation.

\section{CONCLUSIONS AND IMPLICATIONS FOR PRACTICE}

The limited accuracy of CT for the preoperative assessment of tumour spread in patients with advanced ovarian cancer stresses the need for exploration of other preoperative imaging techniques. In this prospective pilot study we found that PET/MRI and DW-MRI are both feasible techniques in preoperative staging of patients with ovarian cancer and have a tendency towards a superior sensitivity for assessment of peritoneal implants in comparison to $\mathrm{CT}$, which could have an impact on treatment decisions. However, results for PET/MRI are mainly a reflection of the contribution of MRI; FDG accumulation was absent in the majority of intraperitoneal tumour sites and could not be used reliably to predict negative tumour sites. DW-MRI on the other hand was able to detect intraperitoneal tumour sites with good diagnostic performance in comparison to $\mathrm{CT}$. The predictive value of DW-MRI regarding ovarian cancer resectability in comparison to $\mathrm{CT}$ has shown promise in recent studies and deserves further investigation in larger prospective trials. DW-MRI is accessible for many clinicians and has the advantage that it does not use ionizing radiation which makes it a suitable option for imaging of patients who have to undergo repeated scans to monitor therapy response. The omission of PET imaging will release restrictions in image acquisition imposed by the integrated $\mathrm{PET} / \mathrm{MRI}$ technique and can substantially reduce examination times. The DW-MRI protocol can be optimized for state-of-the-art MRI devices with motion correction techniques such as MultiVane (Philips Healthcare, Best, the Netherlands) which is more patient-friendly and decreases artefacts without a relevant decrease in diagnostic competence. 


\section{REFERENCES}

$1 \quad$ IKNL (September 2013) Nederlandse Kankerregistratie. Available via http://www.iknl.nl

2 Hoskins WJ, McGuire WP, Brady MF et al (1994) The effect of diameter of largest residual disease on survival after primary cytoreductive surgery in patients with suboptimal residual epithelial ovarian carcinoma. Am J Obstet Gynecol 170:974-979; discussion 979-980

3 Vergote I, Trope CG, Amant F et al (2010) Neoadjuvant chemotherapy or primary surgery in stage IIIC or IV ovarian cancer. N Engl J Med 363:943-953

4 Kehoe S, Hook J, Nankivell M et al (2015) Primary chemotherapy versus primary surgery for newly diagnosed advanced ovarian cancer (CHORUS): an open-label, randomised, controlled, non-inferiority trial. Lancet 386:249-257

5 Qayyum A, Coakley FV, Westphalen AC, Hricak H, Okuno WT, Powell B (2005) Role of CT and MR imaging in predicting optimal cytoreduction of newly diagnosed primary epithelial ovarian cancer. Gynecol Oncol 96:301-306

6 Borley J, Wilhelm-Benartzi C, Yazbek J et al (2015) Radiological predictors of cytoreductive outcomes in patients with advanced ovarian cancer. BJOG 122:843-849

7 Bristow RE, Duska LR, Lambrou NC et al (2000) A model for predicting surgical outcome in patients with advanced ovarian carcinoma using computed tomography. Cancer 89:1532-1540

8 Dowdy SC, Mullany SA, Brandt KR, Huppert BJ, Cliby WA (2004) The utility of computed tomography scans in predicting suboptimal cytoreductive surgery in women with advanced ovarian carcinoma. Cancer 101:346-352

9 Ferrandina G, Sallustio G, Fagotti A et al (2009) Role of CT scan-based and clinical evaluation in the preoperative prediction of optimal cytoreduction in advanced ovarian cancer: a prospective trial. $\mathrm{Br} \mathrm{J}$ Cancer 101:1066-1073

10 Gerestein CG, Eijkemans MJ, Bakker J et al (2011) Nomogram for suboptimal cytoreduction at primary surgery for advanced stage ovarian cancer. Anticancer Res 31:4043-4049

11 Janco JM, Glaser G, Kim B et al (2015) Development of a prediction model for residual disease in newly diagnosed advanced ovarian cancer. Gynecol Oncol 138:70-77

12 Jung DC, Kang S, Kim MJ, Park SY, Kim HB (2010) Multidetector CT predictors of incomplete resection in primary cytoreduction of patients with advanced ovarian cancer. Eur Radiol 20:100-107

13 Jung DC, Kang S, Kim SC et al (2013) Use of complex surgical procedures, patterns of tumor spread, and CA-125 predicts a risk of incomplete cytoreduction: a Korean Gynecologic Oncology Group study (KGOG3022). Gynecol Oncol 131:336-340

14 Kim HJ, Choi CH, Lee YY et al (2014) Surgical outcome prediction in patients with advanced ovarian cancer using computed tomography scans and intraoperative findings. Taiwan J Obstet Gynecol 53:343347

15 Mackintosh ML, Rahim R, Rajashanker B et al (2014) CT scan does not predict optimal debulking in stage III-IV epithelial ovarian cancer: a multicentre validation study. J Obstet Gynaecol 34:424-428

16 Mousavi AS, Mazhari MM, Guilani MM, Ghaemmaghami F, Behtash N, Akhavan S (2010) Can primary optimal cytoreduction be predicted in advanced epithelial ovarian cancer preoperatively? World J Surg Oncol 8:11

17 Stashwick C, Post MD, Arruda JS et al (2011) Surgical risk score predicts suboptimal debulking or a major perioperative complication in patients with advanced epithelial ovarian, fallopian tube, or primary peritoneal cancer. Int J Gynecol Cancer 21:1422-1427

18 Suidan RS, Ramirez PT, Sarasohn DM et al (2014) A multicenter prospective trial evaluating the ability of preoperative computed tomography scan and serum CA-125 to predict suboptimal cytoreduction at primary debulking surgery for advanced ovarian, fallopian tube, and peritoneal cancer. Gynecol Oncol 134:455-461

19 Wahner Hendrickson AE, Hawthorne KM, Goode EL et al (2015) Assessment of published models and prognostic variables in epithelial ovarian cancer at Mayo Clinic. Gynecol Oncol 137:77-85 


\section{Chapter 6}

20 Michielsen K, Vergote I, Op de Beeck K et al (2014) Whole-body MRI with diffusion-weighted sequence for staging of patients with suspected ovarian cancer: a clinical feasibility study in comparison to CT and FDG-PET/CT. Eur Radiol 24:889-901

21 Fujii S, Matsusue E, Kanasaki Y et al (2008) Detection of peritoneal dissemination in gynecological malignancy: evaluation by diffusion-weighted MR imaging. Eur Radiol 18:18-23

22 Lin G, Ho KC, Wang JJ et al (2008) Detection of lymph node metastasis in cervical and uterine cancers by diffusion-weighted magnetic resonance imaging at 3T. J Magn Reson Imaging 28:128-135

23 De laco P, Musto A, Orazi L et al (2011) FDG-PET/CT in advanced ovarian cancer staging: value and pitfalls in detecting lesions in different abdominal and pelvic quadrants compared with laparoscopy. Eur J Radiol 80:e98-103

24 Kumar Dhingra V, Kand P, Basu S (2012) Impact of FDG-PET and -PET/CT imaging in the clinical decisionmaking of ovarian carcinoma: an evidence-based approach. Womens Health (Lond Engl) 8:191-203

25 Partovi S, Kohan A, Rubbert C et al (2014) Clinical oncologic applications of PET/MRI: a new horizon. Am J Nucl Med Mol Imaging 4:202-212

26 Queiroz MA, Kubik-Huch RA, Hauser N et al (2015) PET/MRI and PET/CT in advanced gynaecological tumours: initial experience and comparison. Eur Radiol 25:2222-2230

27 Grueneisen J, Schaarschmidt BM, Beiderwellen K et al (2014) Diagnostic value of diffusion-weighted imaging in simultaneous 18F-FDG PET/MR imaging for whole-body staging of women with pelvic malignancies. J Nucl Med 55:1930-1935

28 Bossuyt PM, Cohen JF, Gatsonis CA, Korevaar DA, group S (2016) STARD 2015: updated reporting guidelines for all diagnostic accuracy studies. Ann Transl Med 4:85

29 Heatley MK (2009) Is female adnexal tumour of probable wolffian origin a benign lesion? A systematic review of the English literature. Pathology 41:645-648

30 Lopez-Lopez V, Cascales-Campos PA, Gil J et al (2016) Use of (18)F-FDG PET/CT in the preoperative evaluation of patients diagnosed with peritoneal carcinomatosis of ovarian origin, candidates to cytoreduction and hipec. A pending issue. Eur J Radiol 85:1824-1828

31 Fagotti A, Vizzielli G, De laco P et al (2013) A multicentric trial (Olympia-MITO 13) on the accuracy of laparoscopy to assess peritoneal spread in ovarian cancer. Am J Obstet Gynecol 209:462 e461-462 e411

32 Rutten MJ, Gaarenstroom KN, Van Gorp T et al (2012) Laparoscopy to predict the result of primary cytoreductive surgery in advanced ovarian cancer patients (LapOvCa-trial): a multicentre randomized controlled study. BMC Cancer 12:31

33 Ataseven B, Grimm C, Harter P et al (2016) Prognostic Impact of Port-Site Metastasis After Diagnostic Laparoscopy for Epithelial Ovarian Cancer. Ann Surg Oncol. 10.1245/s10434-016-5415-9 




\title{
Chapter 7
}

\begin{abstract}
Purpose: Magnetic resonance imaging (MRI) is an important method to evaluate patients with adnexal lesions. Diffusion weighted MRI (DW-MRI) could provide additional diagnostic value. This meta-analysis aims to evaluate the diagnostic accuracy of contrast-enhanced MRI and DW-MRI for differentiation between benign and malignant adnexal masses.
\end{abstract}

Methods: A literature search of studies reporting on the diagnostic performance of MRI or DW-MRI for characterization of adnexal masses was performed. Pooled estimates of sensitivity and specificity derived from regression models were used for meta-analysis. A subgroup analysis was performed for studies which compared MRI and DW-MRI directly.

Results: Thirty-eight datasets were included in the meta-analysis which resulted in pooled sensitivities and specificities of $91 \%$ and $89 \%$ for MRI, and $95 \%$ and $88 \%$ for DWMRI, respectively $(p>.05)$. Four datasets were available for subgroup analysis which resulted in pooled sensitivities and specificities of $87 \%$ and $81 \%$ for MRI, and $94 \%$ and $88 \%$ for DW-MRI, respectively ( $p>.05$ ).

Conclusions: Contrast-enhanced MRI and DW-MRI have high diagnostic accuracy for the characterization of adnexal masses. Addition of DWI to contrast-enhanced MRI showed a positive trend but did not demonstrate a statistically significant improvement in accuracy possibly related to the limited amount of included studies. More prospective studies are desired to establish whether additional DWI can improve diagnosis and treatment of adnexal tumours and alter clinical guidelines. 


\section{INTRODUCTION}

Ovarian cancer remains an important health problem which annually affects a quarter of a million women globally. It has the highest mortality rate of the gynecologic malignancies [1]. Adnexal masses can present as a diagnostic dilemma while a correct differentiation between benign and malignant adnexal lesions is essential for further management. Benign lesions can be treated conservatively or with minimally invasive surgery whereas malignant lesions need more tailored treatment.

Besides ultrasound, secondary imaging techniques such as computed tomography $(\mathrm{CT})$, positron emission tomography (PET) and magnetic resonance imaging (MRI) have been proposed for the assessment of more complex adnexal masses [2]. Reduction of overtreatment and undertreatment by using more reliable imaging techniques could prevent unnecessary morbidity and costs while optimising survival chances. Liu et al compared the diagnostic performance of ultrasound, MRI and CT in a meta-analysis and found no significant differences between the techniques in differentiation between benign and malignant ovarian masses [3]. However, a major drawback of the study was that there were substantially more benign masses in the ultrasound studies which are easier to analyse and therefore the accuracy of both MRI and CT may have been underestimated. In keeping with this assumption, a meta-analysis by Kinkel et al reported that ultrasound was inferior to MRI and CT in the identification of malignancies in a population of women with indeterminate adnexal masses [4]. The most recent meta-analysis which specifically investigated the accuracy of MRI in evaluation of ovarian tumours reported a sensitivity of $92 \%$ and specificity of $85 \%$ [5].

The aforementioned meta-analyses report data from studies that have been performed up until 2009. MRI techniques have evolved since then and functional MRI techniques, such as diffusion weighted imaging (DWI) are being applied more and more in the field of cancer imaging. High signal intensity in the solid portion of an adnexal mass on DWI is correlated with restricted diffusion which can be indicative of malignancy [6]. DWI of mostly cystic adnexal lesions proved to be less useful in distinguishing malignant from benign tumours [7; 8]. The use of ADC (apparent diffusion coefficient) values in the cystic or solid component of the adnexal mass does not contribute to the discrimination between benign and malignant ovarian lesions $[6 ; 9 ; 10]$. Addition of functional techniques such as DWI to conventional anatomical techniques in combined diffusion weighted MRI (DW-MRI) facilitates an improved diagnostic performance of $M R I$ in oncology and individual results have shown promising results [6; 11; 12]. Since many studies evaluating DW-MRI in patients with adnexal masses have been published in the last years, their results are yet to be analysed systematically.

In our opinion a meta-analysis of conventional contrast-enhanced MRI and DW-MRI has high clinical relevance. We hypothesize that adding DWI sequences to the conventional MRI protocol improves the diagnostic performance of MRI in cancer detection. The main objective of this meta-analysis is therefore to evaluate the 


\section{Chapter 7}

diagnostic accuracy of contrast-enhanced MRI and to investigate the added value of DW-MRI for differentiation between benign and malignant adnexal masses.

\section{METHODS}

\section{Protocol and registration}

All methods as described in this manuscript were recorded in a protocol at Prospero under registration number CRD42014009549 (http://www.crd.york.ac.uk/PROSPERO). The conduct of this study was done in accordance with prevailing guidelines (http://www.prisma-statement.org and http://srdta.cochrane.org/handbook-dta-reviews).

\section{Eligibility criteria}

Studies were eligible for inclusion if they were designed as randomized trials of test accuracy or comparative cohort studies reporting on the diagnostic performance of contrast-enhanced MRI and/or DW-MRI in the differentiation between benign and malignant adnexal masses. Studies in which a comparison was made between MRI and/or DW-MRI and alternative diagnostic methods such as US, CT or PET were also included. Findings of these alternative methods were omitted within this analysis. Participants were women diagnosed with at least one adnexal mass who underwent preoperative contrast-enhanced MRI with or without DWI. Studies fulfilling the following criteria were considered: 1) study population of 20 subjects or more, 2) results were given in a $2 \times 2$ contingency table or this table could be derived from the data, 3) histological confirmation was used as reference standard in at least $80 \%$ of patients, 4) prevalence of malignancy was between $15-75 \%$, 5) 1.5 or 3.0 Tesla MRI was

applied, and 6) in DWI studies, signal intensity was subjectively assessed on $b_{1000}$ or $b_{500}$ scans in the solid portion of the adnexal mass and not in the cystic portion. Studies that analyzed specific groups - only benign, only borderline, or only invasive tumors - or studies that were limited to subjects $<18$ years or to pregnant women were excluded.

\section{Search methods and data collection}

A literature search of the Medline, Embase and Scopus databases was performed by two reviewers (IR/SC) independently for studies published between January 1990 and December 2015. This restriction in publication date was applied to ensure an equal comparison between MRI and DWI, since older studies may have used outdated MRI techniques. The search was limited to human subjects and manuscripts that were written in English, Dutch, German or French. The following search terms were used: '(diffusion) magnetic resonance imaging', 'diffusion weighted imaging', '(diffusion) MRI', 
'DW-MRI', 'DWI', 'ovarian neoplasm', 'ovarian cancer' and 'diagnosis'. Both medical subject headings (MeSH) and free text words were used. Reference lists of all relevant articles were checked by hand to find additional studies. The reviewers independently selected eligible studies and assessed their methodological quality by employing the Quality Assessment of Diagnostic Accuracy Studies 2 (QUADAS-2) checklist. A detailed description of this assessment can be found in Online Resource 1. Discrepant findings were settled by a third reviewer (TVG).

\section{Statistical analysis}

To calculate sensitivity and specificity, a $2 \times 2$ contingency table was constructed for each test and each reader within a study. Borderline lesions were classified as malignant for statistical purposes. A bivariate multilevel logistic regression model was used for metaanalysis to account for the functional relationship between sensitivity and specificity within studies [13]. Pooled summary estimates of sensitivity and specificity for each test were calculated with corresponding $95 \%$ confidence intervals $(95 \% \mathrm{Cl})$ based on the fitted model. While most studies only examined a single test, a number of studies compared multiple tests and/or reported results separately for multiple readers based on the same group of patients. We therefore fitted both a two-level model (with correlated random effects for sensitivity and specificity at the test/reader level) and a three-level model (with random effects at the test/reader level and at the study level). However, due to the relatively small number of studies with multiple tests and/or readers, the three-level model did not always converge. Only the results based on the two-level model are therefore reported. This implies that results for multiple tests and/or readers are treated as if they were coming from independent studies, which is incorrect and can lead to incorrect standard errors [14]. Therefore, besides using standard Wald-type tests to compare the mean sensitivity and specificity of the various tests, permutation testing was used to obtain p-values that are in essence distributionfree and exact [15]. For this, the observed test labels (MRI and DW-MRI) were randomly permuted across the different tables 10.000 times. Permutation-based p-values were then obtained by computing the proportion of times that a comparison between two tests (e.g., mean sensitivity of MRI versus DW-MRI) is more extreme under the permutated data than under the actual data.

Based on the summary estimates of sensitivity and specificity from the model, positive and negative likelihood ratios and diagnostic odds ratios were calculated. In addition, summary receiver operating characteristic (SROC) curves were constructed for each test and the corresponding area under the curve (AUC) was calculated. Metaregression analyses were carried out by adding covariates to the bivariate model to assess which factors had a statistically significant relationship with the diagnostic accuracy of the tests. Continuous moderators were dichotomized. The following covariates were investigated: study design (prospective vs. retrospective), mean age 
( $\leq 50$ years vs. $>50$ years), number of readers ( 1 reader vs. $>1$ reader), percentage of borderline lesions ( $\leq 10 \%$ vs. $>10 \%$ ), inclusion vs. exclusion of endometrioma/teratoma, MRI field strength (1.5 Tesla vs. 3.0 Tesla), type of analysis (patient-by-patient vs. lesionby-lesion) and number of patients ( $\leq 100$ patients vs. $>100$ patients).

To prevent bias, one would ideally compare the diagnostic accuracy of MRI and DWMRI only in studies that applied both tests to the same group of patients (or randomized patients to the two tests). A direct comparison between MRI and DW-MRI within a single study was available for 4 datasets. For this subgroup, a separate bivariate multilevel logistic regression model was used to compare summary estimates of sensitivity, specificity, PPV, NPV and DOR. Standard Wald-type tests and permutation tests were used to compare the mean sensitivity and specificity of the two tests. All statistical analyses were performed with Stata v13 (StataCorp. 2013, College Station, Texas, USA) using the xtmelogit command and R v3.1.2 (R Core Team, 2015, Vienna, Austria) using the Ime4 package.

\section{RESULTS}

\section{Study selection}

Results of the literature search are summarized in a flow diagram in Figure 1. After disposing of duplicates, 2433 records were screened on title and abstract and 134 fulltext articles were subsequently assessed for eligibility. Reasons for exclusion are mentioned in detail in Figure 1 and a complete list of excluded articles is available from the authors upon request. Twenty-six studies $[6 ; 9 ; 11 ; 12 ; 16-37]$ fulfilled the inclusion criteria from which 38 data sets were retrieved for MRI $(n=34)$, and DW-MRI $(n=4)$. All data sets combined had been tested on a total of 2082 patients with 2274 adnexal tumors, including 942 (41\%) malignant and 1332 (59\%) benign tumors. An overview of individual study characteristics is presented in Table 1. 


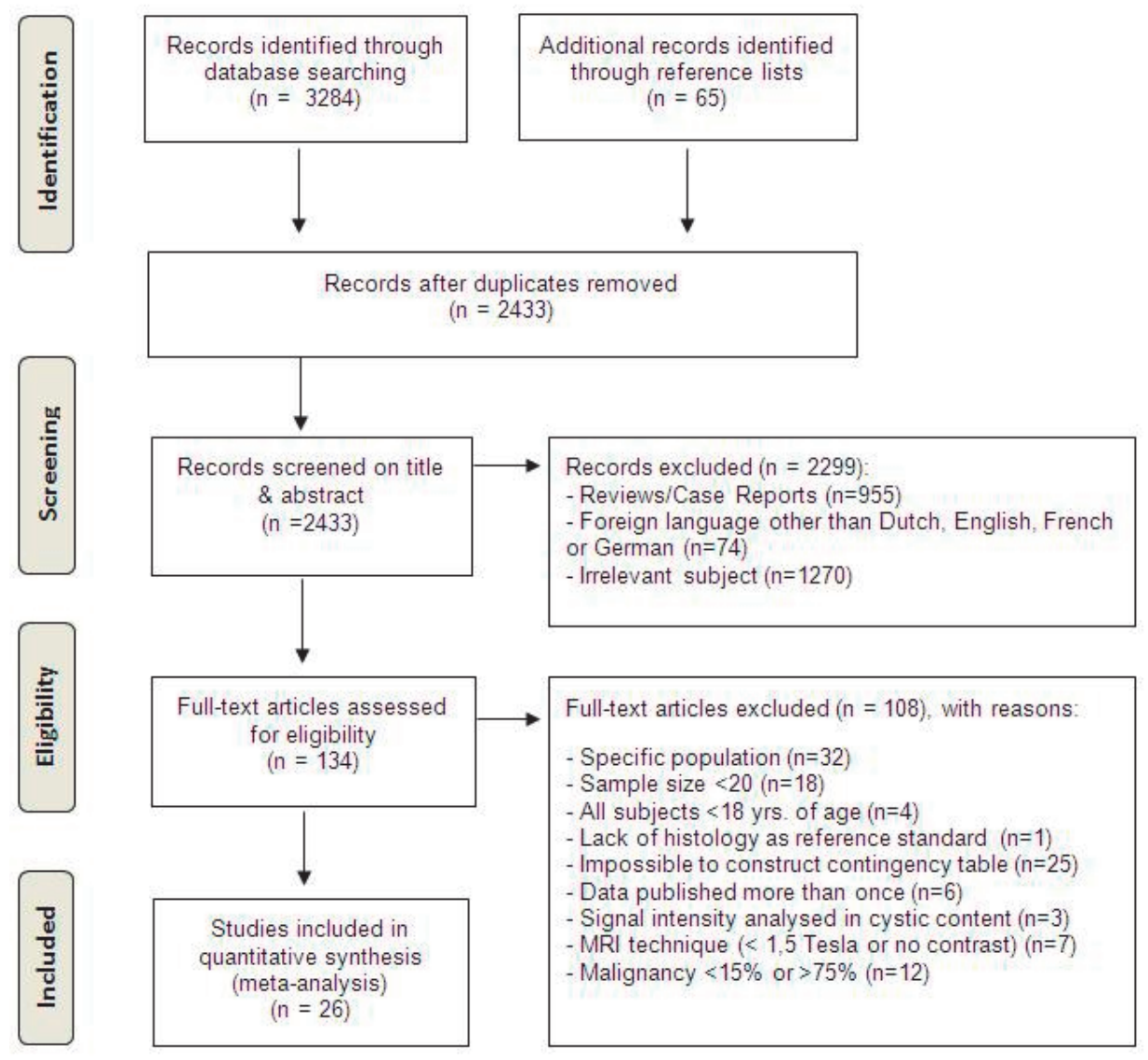

Fig. 1. Flow Diagram (based on PRISMA 2009): selection of studies Abbreviations: $\mathrm{MRI}=$ magnetic resonance imaging 


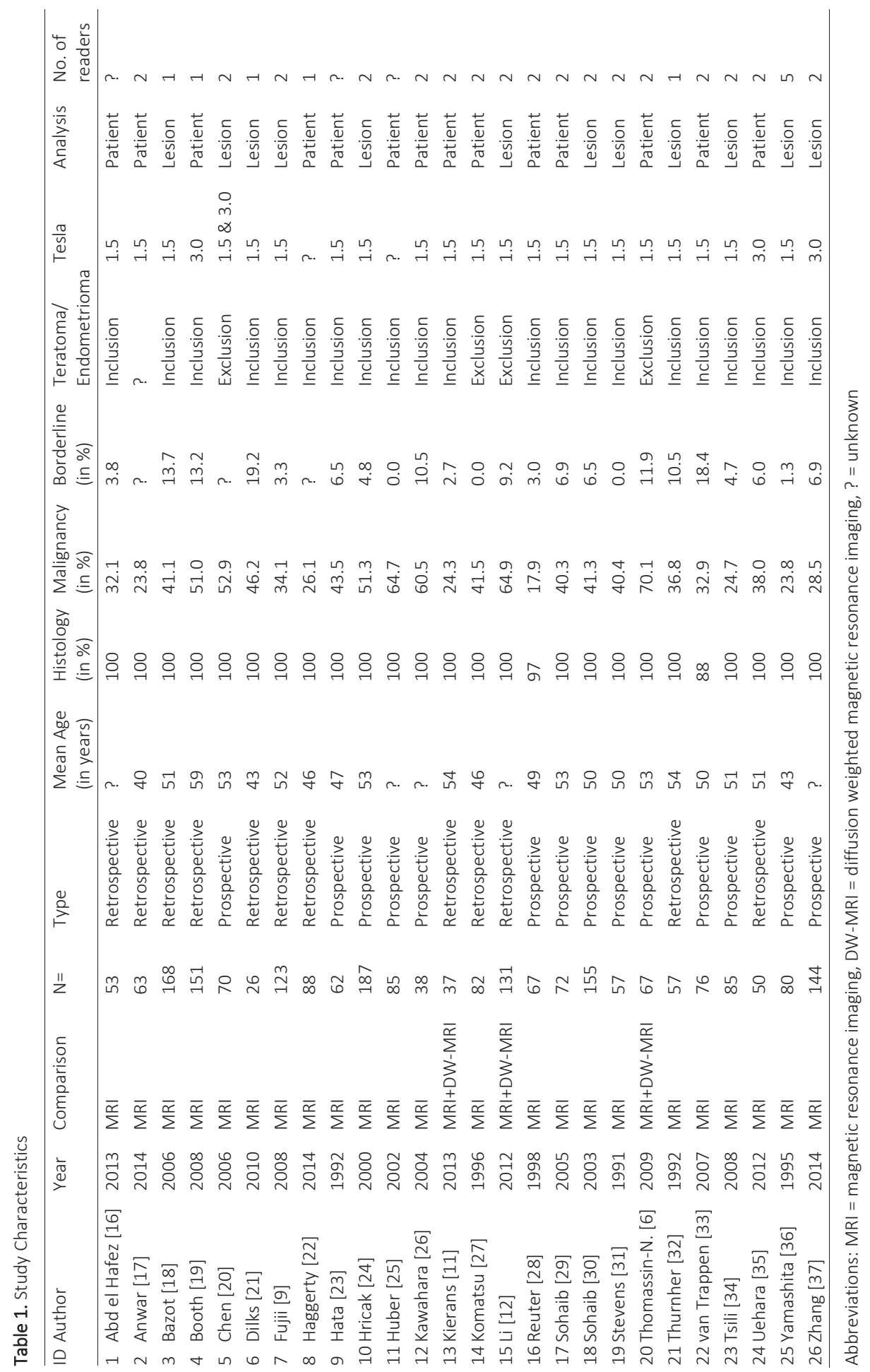




\section{Methodological quality}

Outcome of quality assessment is presented in Table 2. Fifty percent of the studies $(n=13)$ were regarded as 'low' risk of bias and 'low' concern of applicability. Risk of bias regarding the index test was scored 'unclear' 6 times because the explanation of how the index test was interpreted was omitted in several studies. 'High' risk of bias regarding 'Flow and timing' was scored 5 times because not all recruited patients were included in the analysis and this was not recorded or explained separately in all studies. None of the studies were regarded as 'high' risk of bias or concern of applicability when combining the individual assessment areas overall and therefore all 26 studies were included in further statistical analyses.

Table 2. QUADAS-classification per study

\begin{tabular}{|c|c|c|c|c|c|c|c|c|}
\hline \multirow[b]{2}{*}{ Author } & \multirow[b]{2}{*}{ Year } & \multicolumn{4}{|c|}{ Risk of Bias } & \multicolumn{3}{|c|}{ Applicability concerns } \\
\hline & & $\begin{array}{l}\text { Patient } \\
\text { selection }\end{array}$ & Index test & $\begin{array}{l}\text { Reference } \\
\text { standard }\end{array}$ & $\begin{array}{l}\text { Flow and } \\
\text { timing }\end{array}$ & $\begin{array}{l}\text { Patient } \\
\text { selection }\end{array}$ & Index test & $\begin{array}{l}\text { Reference } \\
\text { standard }\end{array}$ \\
\hline Abd el Hafez & 2013 & High & Unclear & Low & Low & High & Low & Low \\
\hline Anwar & 2014 & Low & Unclear & Low & Low & Low & Low & Low \\
\hline Bazot & 2006 & Low & Low & Low & Low & Low & Low & Low \\
\hline Booth & 2008 & Low & Low & Low & Low & Low & Low & Low \\
\hline Chen & 2006 & Low & Low & Low & Low & Low & Low & Low \\
\hline Dilks & 2010 & Low & Low & Low & Low & Low & Low & Low \\
\hline Fujii & 2008 & Low & Unclear & Low & Low & Low & Low & Low \\
\hline Haggerty & 2014 & Low & Low & Low & Low & Low & Unclear & Low \\
\hline Hata & 1992 & Unclear & Unclear & Low & Low & Low & Low & Low \\
\hline Hricak & 2000 & Low & Low & Low & Low & Low & Low & Low \\
\hline Huber & 2002 & Low & Low & Low & High & Low & Unclear & Low \\
\hline Kawahara & 2004 & High & Low & Low & High & Low & Low & Low \\
\hline Kierans & 2013 & Low & Low & Low & Low & Low & Low & Low \\
\hline Komatsu & 1996 & Low & Low & Low & Low & Low & Low & Low \\
\hline Li & 2012 & High & Unclear & Low & Low & Low & Low & Low \\
\hline Reuter & 1998 & Low & Low & Low & Low & Low & Low & Low \\
\hline Sohaib & 2003 & Low & Low & Low & High & Low & Low & Low \\
\hline Sohaib & 2005 & Low & Low & Low & Low & Low & Low & Low \\
\hline Stevens & 1991 & Low & Low & Low & Low & Low & Low & Low \\
\hline Thomassin-N. & 2009 & Low & Unclear & Low & Low & Low & Low & Low \\
\hline Thurnher & 1992 & Low & Low & Low & Low & Low & Low & Low \\
\hline Van Trappen & 2007 & Low & Low & Low & High & Low & Low & Low \\
\hline Tsili & 2008 & Low & Low & Low & High & Low & Low & Low \\
\hline Uehara & 2012 & Unclear & Low & Low & Low & Low & Low & Low \\
\hline Yamashita & 1995 & Low & Low & Low & Low & Low & Low & Low \\
\hline Zhang & 2014 & Low & Low & Low & Low & Low & Low & Low \\
\hline
\end{tabular}

High/Low/Unclear describes the risk of bias per study and per category. 


\section{Chapter 7}

\section{Findings}

Sensitivity and specificity with $95 \% \mathrm{Cl}$ are presented as forest plots in Figure 2. Results of the pooled sensitivity and specificity based on the bivariate model incorporating all studies are shown in Table 3. Sensitivities for MRI and DW-MRI were 91\% [95\%Cl 88-93] and $95 \%$ [95\% $\mathrm{Cl} 89-98]$, respectively. These sensitivities were not significantly different ( $p=.166$ for the standard Wald-type test and $p=.194$ for the permutation test). Specificities for MRI and DW-MRI were $89 \%$ [95\% Cl 87-91] and 88\% [95\% $\mathrm{Cl} 78-94$ ], respectively. The difference in specificity between $\mathrm{MRI}$ and DW-MRI was also not significant ( $p=0.765$ for the Wald-type test and $p=.749$ for the permutation test).

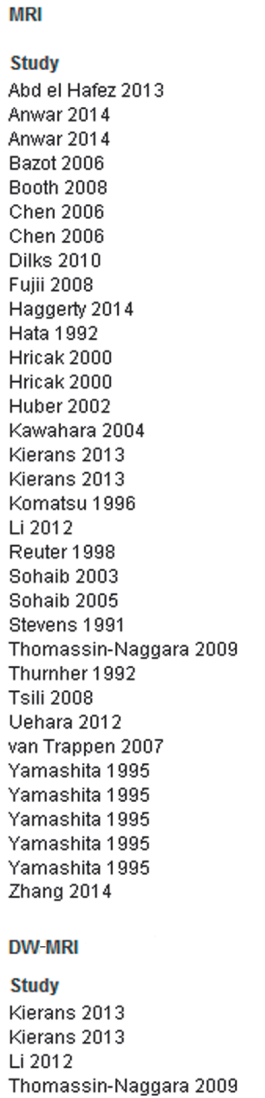

$\begin{array}{rrrrr}\text { TP } & \text { FP } & \text { FN } & \text { TN } & \text { Sensitivity }(95 \% \text { Cl) } \\ 16 & 3 & 1 & 33 & 0.94[0.71,1.00] \\ 14 & 2 & 1 & 46 & 0.93[0.68,1.00] \\ 14 & 2 & 1 & 46 & 0.93[0.68,1.00] \\ 58 & 8 & 11 & 91 & 0.84[0.73,0.92] \\ 71 & 18 & 6 & 56 & 0.92[0.84,0.97] \\ 32 & 4 & 5 & 29 & 0.86[0.71,0.95] \\ 30 & 6 & 7 & 27 & 0.81[0.65,0.92] \\ 12 & 3 & 0 & 11 & 1.00[0.74,1.00] \\ 40 & 8 & 2 & 73 & 0.95[0.84,0.99] \\ 19 & 9 & 4 & 56 & 0.83[0.61,0.95] \\ 18 & 1 & 9 & 34 & 0.67[0.46,0.83] \\ 87 & 6 & 9 & 69 & 0.91[0.83,0.96] \\ 84 & 11 & 12 & 64 & 0.88[0.79,0.93] \\ 52 & 4 & 3 & 26 & 0.95[0.85,0.99] \\ 21 & 2 & 2 & 13 & 0.91[0.72,0.99] \\ 6 & 7 & 3 & 21 & 0.67[0.30,0.93] \\ 8 & 0 & 1 & 28 & 0.89[0.52,1.00] \\ 31 & 6 & 3 & 42 & 0.91[0.76,0.98] \\ 78 & 10 & 7 & 36 & 0.92[0.84,0.97] \\ 12 & 12 & 0 & 43 & 1.00[0.74,1.00] \\ 61 & 11 & 3 & 80 & 0.95[0.87,0.99] \\ 28 & 7 & 1 & 36 & 0.97[0.82,1.00] \\ 23 & 4 & 0 & 30 & 1.00[0.85,1.00] \\ 42 & 8 & 5 & 12 & 0.89[0.77,0.96] \\ 20 & 2 & 1 & 34 & 0.95[0.76,1.00] \\ 20 & 1 & 1 & 63 & 0.95[0.76,1.00] \\ 18 & 3 & 1 & 28 & 0.95[0.74,1.00] \\ 23 & 7 & 2 & 44 & 0.92[0.74,0.99] \\ 17 & 2 & 2 & 59 & 0.89[0.67,0.99] \\ 17 & 4 & 2 & 57 & 0.89[0.67,0.99] \\ 17 & 3 & 2 & 58 & 0.89[0.67,0.99] \\ 17 & 6 & 2 & 55 & 0.89[0.67,0.99] \\ 18 & 6 & 1 & 55 & 0.95[0.74,1.00] \\ 38 & 11 & 3 & 92 & 0.93[0.80,0.98] \\ & & & & \end{array}$
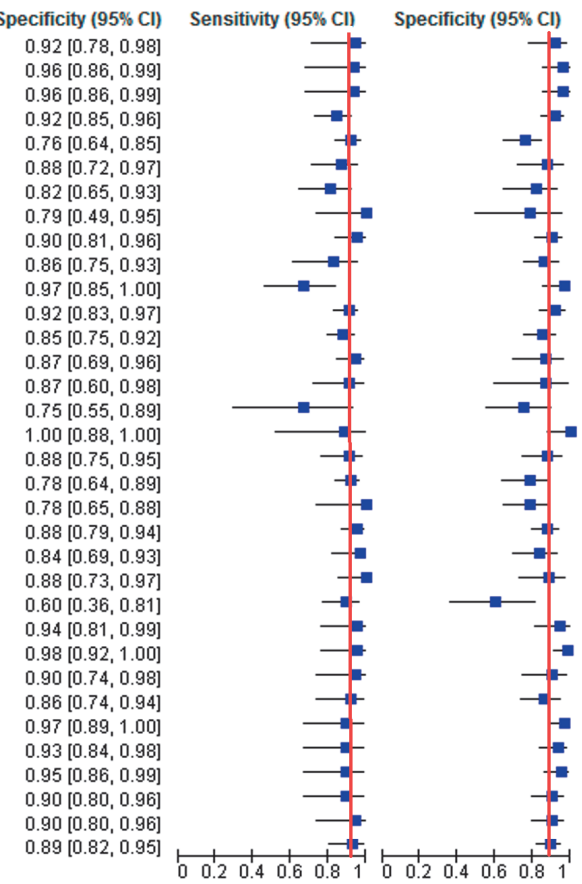

$\begin{array}{rrrrr}\text { TP } & \text { FP } & \text { FN } & \text { TN } & \text { Sensitivity }(95 \% \text { Cl) } \\ 6 & 4 & 3 & 24 & 0.67[0.30,0.93] \\ 8 & 1 & 1 & 27 & 0.89[0.52,1.00] \\ 82 & 5 & 3 & 41 & 0.96[0.90,0.99] \\ 47 & 5 & 0 & 15 & 1.00[0.92,1.00]\end{array}$

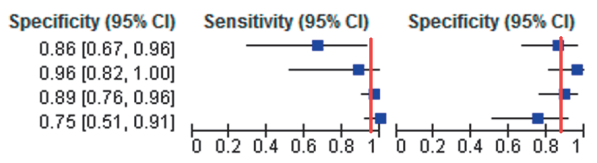

Fig. 2. Forest Plots of sensitivity and specificity of MRI and DW-MRI in the differentiation of benign and malignant adnexal masses

Abbreviations: $\mathrm{MRI}=$ magnetic resonance imaging, DW-MRI = diffusion weighted magnetic resonance imaging, $\mathrm{TP}=$ true positive, $\mathrm{FP}=$ false positive, $\mathrm{FN}=$ false negative, $\mathrm{TN}=$ true negative, $\mathrm{Cl}=$ confidence interval. Red reference lines indicate estimates for pooled summary sensitivities and specificities 
Table 3. Pooled summary measures (A) for all studies that investigated MRI and/or DW-MRI and (B) for subgroup studies that compared MRI and DW-MRI directly

\begin{tabular}{lllll}
\hline Parameter & MRI & DW-MRI & $\begin{array}{l}\text { P-value } \\
\text { (Wald-type test) }\end{array}$ & $\begin{array}{l}\text { P-value } \\
\text { (permutation test) }\end{array}$ \\
\hline A. All studies & & & \\
Sensitivity in \% (95\%Cl) & $91(88-93)$ & $95(89-98)$ & 0.166 & 0.194 \\
Specificity in \% (95\%Cl) & $89(87-91)$ & $88(78-94)$ & 0.765 & 0.749 \\
PLR (95\%Cl) & $8.49(6.87-10.48)$ & $7.99(4.15-15.41)$ & 0.865 & 0.852 \\
NLR $(95 \% \mathrm{Cl})$ & $0.10(0.08-0.13)$ & $0.06(0.02-0.13)$ & 0.180 & 0.201 \\
DOR $(95 \% \mathrm{Cl})$ & $81.60(58.38-114.05)$ & $140.29(46.45-423.74)$ & 0.362 & 0.419 \\
B. Subgroup Analysis & & & & 0.342 \\
Sensitivity in \% (95\%Cl) & $87(73-95)$ & $94(85-98)$ & 0.227 & 0.528 \\
Specificity in \% (95\%Cl) & $81(65-91)$ & $88(76-95)$ & 0.355 & 0.411 \\
PLR (95\%Cl) & $4.65(2.37-9.13)$ & $8.16(3.76-17.72)$ & 0.277 & 0.253 \\
NLR $(95 \% \mathrm{Cl})$ & $0.15(0.07-0.35)$ & $0.06(0.02-0.18)$ & 0.177 & 0.197 \\
DOR (95\%Cl) & $30.07(9.50-95.22)$ & $131.16(35.57-483.61)$ & 0.094 & \\
\hline
\end{tabular}

Abbreviations: $\mathrm{MRI}=$ magnetic resonance imaging, DW-MRI = diffusion weighted magnetic resonance imaging, PLR=positive likelihood ratio, NLR=negative likelihood ratio, DOR=diagnostic odds ratio

Summary ROC curves are presented in Figure 3. The AUCs based on these curves were equal to 0.95 and 0.97 for MRI and DW-MRI, respectively.

Meta-regression analyses showed no significant differences in sensitivity between any of the covariates (Table 4). Significant differences in specificity were present: studies which included fewer borderline tumors reported a higher specificity $(p=.020)$. A higher specificity was also noted for studies in which patients with teratoma and/or endometrioma were included compared to studies in which patients with these lesions were excluded $(p=.010)$.

Four datasets from three studies were available for subgroup analysis from which the results are presented in Table 3. DW-MRI showed a higher diagnostic performance to $\mathrm{MRI}$ alone expressed as a sensitivity of $94 \%$ (compared to $87 \%$ for MRI) and specificity of $88 \%$ (compared to $81 \%$ for MRI). The differences in sensitivity ( $p=.227$ for the Wald-type test and $p=.342$ for the permutation test) and specificity ( $p=.355$ for the Wald-type test and $p=0.528$ for the permutation test) were not statistically significant. Differences in PLR (8.16 for DW-MRI vs. 4.65 for MRI), NLR (0.06 for DW-MRI vs. 0.15 for MRI) and DOR (131.16 for DW-MRI vs. 30.07 for MRI) were also not statistically significant. 


\section{Chapter 7}

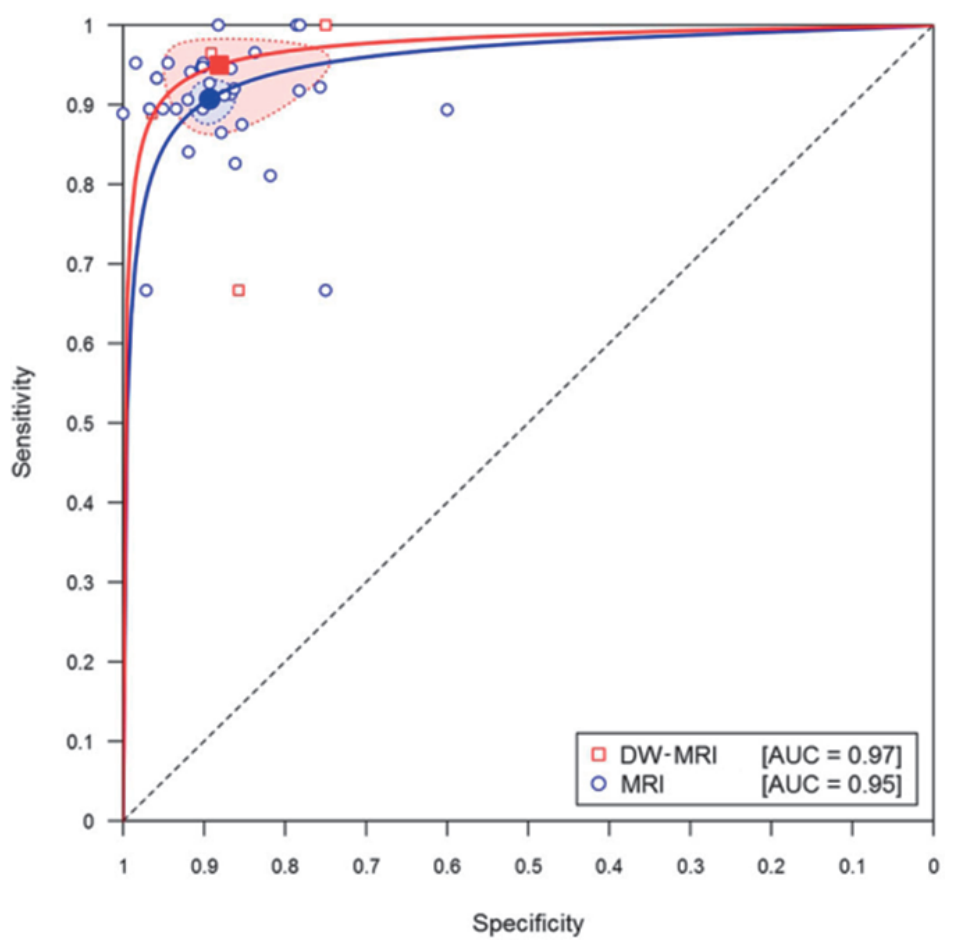

Fig. 3. Summary receiver operating characteristic (SROC) plots for MRI and DW-MRI with $95 \%$ confidence regions

Abbreviations: $\mathrm{MRI}=$ magnetic resonance imaging, DW-MRI = diffusion weighted magnetic resonance imaging, $A \cup C=$ Area under the curve

Table 4. Covariate analysis of all studies that investigated MRI and/or DW-MRI

\begin{tabular}{|c|c|c|c|c|c|}
\hline & Covariate & Sensitivity (\%) & P-value & Specificity (\%) & P-value \\
\hline A & $\begin{array}{l}\text { Retrospective } \\
\text { Prospective }\end{array}$ & $\begin{array}{l}91 \\
91\end{array}$ & 0.868 & $\begin{array}{l}89 \\
89\end{array}$ & 0.884 \\
\hline B & $\begin{array}{l}\text { Mean age } \leq 50 \text { years } \\
\text { Mean age }>50 \text { years }\end{array}$ & $\begin{array}{l}91 \\
90\end{array}$ & 0.595 & $\begin{array}{l}91 \\
88\end{array}$ & 0.228 \\
\hline C & $\begin{array}{l}1 \text { reader } \\
>1 \text { reader }\end{array}$ & $\begin{array}{l}89 \\
92\end{array}$ & 0.330 & $\begin{array}{l}87 \\
89\end{array}$ & 0.473 \\
\hline D & $\begin{array}{l}\text { Borderline } \leq 10 \% \\
\text { Borderline }>10 \%\end{array}$ & $\begin{array}{l}92 \\
93\end{array}$ & 0.696 & $\begin{array}{l}90 \\
83\end{array}$ & $0.020^{*}$ \\
\hline$E$ & $\begin{array}{l}\text { Teratoma excluded } \\
\text { Teratoma included }\end{array}$ & $\begin{array}{l}92 \\
91\end{array}$ & 0.649 & $\begin{array}{l}82 \\
90\end{array}$ & $0.010^{*}$ \\
\hline $\mathrm{F}$ & $\begin{array}{l}\text { 1.5 Tesla } \\
\text { 3.0 Tesla }\end{array}$ & $\begin{array}{l}92 \\
93\end{array}$ & 0.678 & $\begin{array}{l}90 \\
86\end{array}$ & 0.284 \\
\hline G & $\begin{array}{l}\text { Lesion-based } \\
\text { Patient-based }\end{array}$ & $\begin{array}{l}92 \\
91\end{array}$ & 0.601 & $\begin{array}{l}91 \\
87\end{array}$ & 0.117 \\
\hline $\mathrm{H}$ & $\begin{array}{l}\leq 100 \text { patients } \\
>100 \text { patients }\end{array}$ & $\begin{array}{l}91 \\
92\end{array}$ & 0.581 & $\begin{array}{l}90 \\
88\end{array}$ & 0.346 \\
\hline
\end{tabular}

* Indicates statistical significance with a p-value $<.05$ 


\section{DISCUSSION}

\section{Summary of main results}

This meta-analysis on the diagnostic value of contrast-enhanced MRI and DW-MRI for differentiating benign and malignant adnexal masses resulted in a mean sensitivity and specificity of $91 \%$ and $90 \%$ for MRI, and $95 \%$ and $88 \%$ for DW-MRI in meta-analysis of 38 datasets. High AUC-values were observed for both MRI (AUC $=0.95$ ) as well as DWMRI (AUC=0.97). In subgroup analysis of the 4 datasets that compared MRI and DW-MRI directly, a higher diagnostic performance was observed for DW-MRI. However, this difference was not statistically different.

The results for MRI (without DWI) are in accordance with previous meta-analyses that investigated the role of MRI as a diagnostic tool for adnexal masses. Medeiros et al found a sensitivity of $92 \%$ and a specificity of $85 \%$ for MRI detection of borderline or invasive ovarian tumors with an AUC of 0.95 [5]. Dodge et al confirmed these findings by reporting comparable values for sensitivity and specificity, $92 \%$ and $88 \%$ respectively [38]. However, a substantial number of studies have been published since and the current meta-analysis provides an update of the literature with a large amount of datasets ( $n=34$ ) for MRI. In comparison, the most recent meta-analysis of Medeiros et al only used 18 datasets [5]. Twelve datasets used in the publication of Medeiros et al were included in this meta-analysis as well. Six datasets could not be included due to differences in eligibility criteria.

A trend was seen in our subgroup analysis that DW-MRI has superior diagnostic accuracy over conventional MRI although the differences in sensitivity, specificity, PLR, NLR and DOR were not statistically significant. We feel that the small number of included studies likely contributed to this. Nevertheless these results might demonstrate a potential clinically relevant improvement in accuracy. The addition of DWI to conventional contrast-enhanced MRI adds functional information to the anatomical information and has the potential to prevent false positive findings (hence the higher specificity and PLR) and false negative findings (reflecting in higher sensitivity and better NLR). However, when we reviewed the included studies it was noticed that the accuracy of the conventional MRI might be underestimated. In one particular study only the T2 signal intensity without any subjective assessment of other radiologic features was used to predict malignancy on MRI [6]. The value for specificity in this dataset was considerably lower in comparison to the reported specificities from the other three datasets. Inclusion of standard subjective assessment alongside signal intensity measurement would probably have resulted in fewer positive results and a higher specificity for MRI.

As mentioned in the results section, studies which included patients with teratoma and/or endometrioma showed a higher specificity than studies in which patients with these lesions were excluded. This difference can be explained by the fact that these 
teratomas and endometriomas are fairly 'easy to diagnose' on conventional MRI although they are often problematic for DWI. An abnormal signal intensity can be seen on DWI for these lesions, which is suggested to be caused by keratinous substance in teratomas and intra-cystic blood clots in endometriomas [9; 10]. The inclusion or exclusion of these lesions influences MRI and DWI anticipated results accordingly.

On the other hand the inclusion of more borderline tumors introduces more 'difficult diagnoses'. In our meta-analysis we classified borderline tumors as malignant but they can be easily mistaken for benign tumors since they can possess both benign and malignant characteristics on imaging. We therefore expected this to result in a higher amount of false negatives for studies including large numbers of borderline tumors. Surprisingly, we found no differences in sensitivity but a significantly lower specificity in our covariate analysis for studies that included $>10 \%$ borderline tumors.

\section{Limitations}

The main limitation of this meta-analysis is the decreased power to detect true differences in diagnostic accuracy between the two tests due to the relative low number of studies that studied DW-MRI. DW-MRI is a relatively new technique and has to date not been incorporated into standard clinical practice, especially in smaller local hospitals. The present results, especially with respect to DW-MRI, therefore need to be handled with some caution. By including studies which evaluated only one of the tests of interest we maximized the number of studies in the analyses as to produce the most reliable results. In this way we gathered a substantial amount of datasets to evaluate the diagnostic performance of MRI and we were able to carry out a covariate analysis. We handled the multiplicity of tests and readers in the present meta-analysis by using permutation testing when comparing the diagnostic value of the different tests.

Although we maintained strict selection criteria to minimize heterogeneity between studies, methodological issues and study design deserve further comment. Two reviewers independently selected and extracted data and assessed the quality of the included studies with a certified tool. Because we were looking to answer a specific question for a specific group, our inclusion and exclusion criteria were designed to allow for the assembly of a compatible population. Histological confirmation in at least $80 \%$ of patients and a study population of 20 subjects or more was chosen as the absolute minimum to be able to construct reliable contingency tables. Additionally, the choice was made to include studies with a prevalence of malignancy between 15-75\% of the population. Though arbitrary, this decision was made to ensure an inclusion of studies where MRI was performed for a similar indication. The inclusion of studies with an exceedingly low number of true positives (low percentage of malignancy) or true negatives (high percentage of malignancy) may result in an over- or underestimation of the diagnostic performance. Measurements of the signal intensity on DWI in the cystic portion of an adnexal mass compared with measurements in the solid portion have not 
proven useful in differentiation between benignity and malignancy [7; 8]. For this reason and to enable equal comparison within DWI studies, only the studies that made use of the solid portion of the masses were included. Owing to the nature of metaanalyses in general, some heterogeneity between studies is bound to exist based on design characteristics. We tried to account for this heterogeneity by testing different study characteristics in meta-regression analyses. We also added population size as a variable in our covariate analysis to detect publication bias. We found no significant difference in sensitivity or specificity between studies with a smaller or larger population and therefore we assume that the risk of publication bias is low.

\section{Authors' conclusions}

Meta-analysis of 38 datasets showed good diagnostic accuracy for contrast-enhanced MRI and combined DW-MRI in the discrimination of benign from malignant adnexal masses. The addition of DWI to MRI showed a positive trend towards an improvement in diagnostic performance compared to MRI alone. Although this improvement was not statistically significant we believe that the use of DWI can still be clinically relevant. We assume that significance was not reached due to a lack of power conferred by a limited number of patients in a limited amount of studies that examined DW-MRI. More prospective studies on the combination of DW-MRI are desired to confirm the present findings and to establish whether complementing conventional MRI with DWI has the possibility to improve the diagnosis and treatment of women with adnexal tumors. 


\section{Chapter 7}

\section{SUPPLEMENTARY MATERIAL}

Assessment of QUADAS-2 Checklist

Domain 1: Patient selection

\section{A. RISK OF BIAS}

Was a consecutive or random sample of patients enrolled?

a. Yes: All eligible patients suspected of having the target condition during a specific period, or random samples of those patients were enrolled. The investigators had no freedom of choice as to which individual patients were included.

b. No: A non-consecutive sample of patients was enrolled or the investigators had influence on the choice which individual patients were included.

c. Unclear: Patient selection was unclear, study does not mention number of eligible patients or their method of selection.

Was a case-control design avoided?

Studies with a case-control design were excluded from this meta-analysis. "Yes" was scored for all studies.

Did the study avoid inappropriate exclusions?

a. Yes: inappropriate exclusions are avoided, different conditions and different patients are included.

b. No: some inappropriate exclusions are made which may result in either overestimates of underestimates of diagnostic accuracy. Example: exclusion of patients with borderline malignancy.

c. Unclear: In- and exclusion process was unclear.

Could the selection of patients have introduced bias?

- Low risk: all signalling questions for a domain are answered with "yes" or 2 questions are answered with "yes" and one questions is answered with "unclear".

- High risk: 1 or more signalling questions for a domain are answered with "no".

- Unclear: Insufficient data are reported to permit a judgment, 2 signalling questions are answered with "unclear" and the question on case-control with "yes" by default.

\section{B. Applicability}

Are there concerns that the included patients do not match the review question?

- Low concern: patients match the review question based on severity of target condition, demographic features, presence of differential diagnosis of co-morbidity, setting of the study and previous testing protocols.

- High concern: studies show clear deviations from the review question based on severity of target condition, demographic features, presence of differential diagnosis of co-morbidity, setting of the study and previous testing protocols.

- Unclear: Insufficient data are reported to permit a judgment.

NB: Since the studies were thoroughly judged on inclusion and exclusion criteria, almost all studies were scored "low concern" and no studies were scored "high concern". 
Domain 2: Index test

\section{A. RISK OF BIAS}

Were the index test results interpreted without knowledge of the results of the reference standard?

a. Yes: interpretation of the index test was performed before results of the reference standard were available or the investigator had no knowledge of the outcome of the reference standard, blinding was applied.

b. No: interpretation of the index test was performed with knowledge of the outcome of the reference standard, no blinding was applied.

c. Unclear: it is unclear if the index test results were interpreted without knowledge of the results of the reference standard.

If a threshold was used, was it pre-specified?

This question was not applicable to our review question and was therefore omitted.

Could the conduct or interpretation of the index test have introduced bias?

- Low risk: the signalling question is answered with "yes".

- High risk: the signalling question is answered with "no".

- Unclear: the signalling question is answered with "unclear".

\section{B. Applicability}

Are there concerns that the index test, its conduct, or interpretation differ from the review question?

- Low concern: Technology, execution and interpretation of the index test are similar to what is used in daily practice with the target population. Variations are not likely to affect estimates of diagnostic accuracy.

- High concern: Technology, execution and interpretation of the index test are dissimilar to what is used in daily practice with the target population. Variations are likely to affect estimates of diagnostic accuracy.

- Unclear: Insufficient data are reported to permit a judgment.

\section{Domain 3: Reference standard}

\section{A. RISK OF BIAS}

Is the reference standard likely to correctly classify the target condition?

One of our inclusion criteria stated that histological confirmation had to be used as reference standard in at least $80 \%$ of the patients. Histological confirmation is highly likely to correctly identify the target condition, therefore all studies were scored "yes" for this question.

Were the reference standard results interpreted without knowledge of the results of the index test?

a. Yes: the reference standard was applied without any prior knowledge of the outcome of previous tests.

b. No: the reference standard was applied with prior knowledge of the outcome of previous tests.

c. Unclear: it is not stated in the study if the reference standard was applied with knowledge of the outcome of previous tests.

Since all but one study did not report any information on how the reference standard was applied, 25 studies were scored "unclear" and one study was scored "yes". It was not possible to obtain this information from the studies.

Could the reference standard, its conduct, or its interpretation have introduced bias?

Since all studies scored "yes" on the first question and "unclear" or yes on the second question, all studies were scored "low risk". 


\section{Chapter 7}

\section{B. Applicability}

\section{Are there concerns that the target condition as defined by the reference standard does not match the review question? \\ The same reference standard was used in all studies (histology) and this reference standard was assumed to correctly define the target condition, therefore all studies were scored as "low concern"}

Domain 4: Flow and timing

\section{RISK OF BIAS}

Was there an appropriate interval between index test and reference standard?

a. Yes: the interval between index test and reference standard was 120 days or less for the majority of patients.

b. No: the interval between index test and reference standard was more than 120 days for a substantial amount of patients.

c. Unclear: the interval between index test and reference standard is unclear.

NB: the time interval of 120 days is a subject of discussion. The index test and reference standard are ideally performed close together in time but this is not always possible since surgery has to be planned in advance and some hospitals have problems with waiting lists. However, the time interval should not exceed more than 3-4 months to our opinion because new tumours (benign or malign) may have formed whether or not originating from the primary lesion or benign lesions may have regressed and are not visible anymore at time of surgery.

Did all patients receive a reference standard?

a. Yes: all patients received a reference standard from which a diagnosis could be deducted.

b. No: a number of patients did not receive a reference standard from which a diagnosis could be deducted.

c. Unclear: it is unclear whether all patients received a reference standard from which a diagnosis could be deducted.

Did patients receive the same reference standard?

a. Yes: less than $20 \%$ of patients did not receive histology as reference standard.

b. No: more than $20 \%$ of patients did not receive histology as reference standard.

c. Unclear: it is unclear whether all patients received the same reference standard.

One of our inclusion criteria stated that histological confirmation had to be used as reference standard in at least $80 \%$ of the patients. Therefore we scored "yes" for all studies.

Were all patients included in the analysis?

a. Yes: all recruited patients are included in the analysis. Patients who are not included in the analysis because no index test or reference standard was obtained are recorded and explained separately.

b. No: not all recruited patients are included in the analysis. Patients who are not included in the analysis because no index test or reference standard was obtained are not recorded or explained separately.

c. Unclear: it is unclear whether all recruited patients are included in the analysis. It is unclear if there are patients who are not included in the analysis because no index test or reference standard was obtained.

Could the patient flow have introduced bias?

- Low risk: 3 or more signalling are answered with "yes", no questions are answered with "no"

- High risk: 1 or more signalling questions for a domain are answered with "no".

- Unclear: Insufficient data are reported to permit a judgment. 3 or more signalling questions are answered with "unclear", no questions are answered with "no". 


\section{REFERENCES}

1 Ferlay J, Soerjomataram I, Ervik M et al GLOBOCAN 2012 v1.0, Cancer Incidence and Mortality Worldwide: IARC CancerBase No. 11, 2013.

2 Kaijser J, Sayasneh A, Van Hoorde K et al (2014) Presurgical diagnosis of adnexal tumours using mathematical models and scoring systems: a systematic review and meta-analysis. Hum Reprod Update 20:449-462

3 Liu J, Xu Y, Wang J (2007) Ultrasonography, computed tomography and magnetic resonance imaging for diagnosis of ovarian carcinoma. Eur J Radiol 62:328-334

4 Kinkel K, Lu Y, Mehdizade A, Pelte MF, Hricak H (2005) Indeterminate ovarian mass at US: incremental value of second imaging test for characterization--meta-analysis and Bayesian analysis. Radiology 236:85-94

5 Medeiros LR, Freitas LB, Rosa DD et al (2011) Accuracy of magnetic resonance imaging in ovarian tumor: a systematic quantitative review. Am J Obstet Gynecol 204:67 e61-10

6 Thomassin-Naggara I, Darai E, Cuenod CA et al (2009) Contribution of diffusion-weighted MR imaging for predicting benignity of complex adnexal masses. Eur Radiol 19:1544-1552

7 Cappabianca S, laselli F, Reginelli A et al (2013) Value of diffusion-weighted magnetic resonance imaging in the characterization of complex adnexal masses. Tumori 99:210-217

8 Nakayama T, Yoshimitsu K, Irie H et al (2005) Diffusion-weighted echo-planar MR imaging and ADC mapping in the differential diagnosis of ovarian cystic masses: usefulness of detecting keratinoid substances in mature cystic teratomas. J Magn Reson Imaging 22:271-278

9 Fujii S, Kakite S, Nishihara K et al (2008) Diagnostic accuracy of diffusion-weighted imaging in differentiating benign from malignant ovarian lesions. J Magn Reson Imaging 28:1149-1156

10 Katayama M, Masui T, Kobayashi S et al (2002) Diffusion-weighted echo planar imaging of ovarian tumors: is it useful to measure apparent diffusion coefficients? J Comput Assist Tomogr 26:250-256

11 Kierans AS, Bennett GL, Mussi TC et al (2013) Characterization of malignancy of adnexal lesions using ADC entropy: comparison with mean $A D C$ and qualitative DWI assessment. J Magn Reson Imaging 37:164-171

12 Li W, Chu C, Cui Y, Zhang P, Zhu M (2012) Diffusion-weighted MRI: a useful technique to discriminate benign versus malignant ovarian surface epithelial tumors with solid and cystic components. Abdom Imaging 37:897-903

13 Hamza TH, Reitsma JB, Stijnen T (2008) Meta-analysis of diagnostic studies: a comparison of random intercept, normal-normal, and binomial-normal bivariate summary ROC approaches. Med Decis Making 28:639-649

14 Macaskill P, Gatsonis C, Deeks JJ, Harbord RM, Takwoingi Y Chapter 10: Analysing and Presenting Results. In: Deeks JJ, Bossuyt PM, Gatsonis C (editors), Cochrane Handbook for Systematic Reviews of Diagnostic Test Accuracy Version 10 The Cochrane Collaboration, 2010

15 Good P (2005) Permutation, parametric and bootstrap tests of hypotheses (3rd ed.). Springer, New York

16 Abd El hafez A, Monir A (2013) Diagnostic spectrum of ovarian masses in women with breast cancer; magnetic resonance imaging: histopathology correlation. Ann Diagn Pathol 17:441-447

17 Anwar S, Rehan B, Hameed G (2014) MRI for the diagnosis of ultrasonographically indeterminate pelvic masses. J Pak Med Assoc 64:171-174

18 Bazot M, Nassar-Slaba J, Thomassin-Naggara I, Cortez A, Uzan S, Darai E (2006) MR imaging compared with intraoperative frozen-section examination for the diagnosis of adnexal tumors; correlation with final histology. Eur Radiol 16:2687-2699

19 Booth SJ, Turnbull LW, Poole DR, Richmond I (2008) The accurate staging of ovarian cancer using 3T magnetic resonance imaging--a realistic option. BJOG 115:894-901

20 Chen M, Wang WC, Zhou C et al (2006) Differentiation between malignant and benign ovarian tumors by magnetic resonance imaging. Chin Med Sci J 21:270-275 


\section{Chapter 7}

21 Dilks P, Narayanan P, Reznek R, Sahdev A, Rockall A (2010) Can quantitative dynamic contrast-enhanced MRI independently characterize an ovarian mass? Eur Radiol 20:2176-2183

22 Haggerty AF, Hagemann AR, Chu C, Siegelman ES, Rubin SC (2014) Correlation of pelvic magnetic resonance imaging diagnosis with pathology for indeterminate adnexal masses. Int J Gynecol Cancer 24:1215-1221

23 Hata K, Hata T, Manabe A, Sugimura K, Kitao M (1992) A critical evaluation of transvaginal Doppler studies, transvaginal sonography, magnetic resonance imaging, and CA 125 in detecting ovarian cancer. Obstet Gynecol 80:922-926

24 Hricak H, Chen M, Coakley FV et al (2000) Complex adnexal masses: detection and characterization with MR imaging--multivariate analysis. Radiology 214:39-46

25 Huber S, Medl M, Baumann L, Czembirek H (2002) Value of ultrasound and magnetic resonance imaging in the preoperative evaluation of suspected ovarian masses. Anticancer Res 22:2501-2507

26 Kawahara K, Yoshida Y, Kurokawa T et al (2004) Evaluation of positron emission tomography with tracer 18-fluorodeoxyglucose in addition to magnetic resonance imaging in the diagnosis of ovarian cancer in selected women after ultrasonography. J Comput Assist Tomogr 28:505-516

27 Komatsu T, Konishi I, Mandai M et al (1996) Adnexal masses: transvaginal US and gadolinium-enhanced MR imaging assessment of intratumoral structure. Radiology 198:109-115

28 Reuter M, Steffens J, Schuppler U et al (1998) Critical evaluation of the specificity of MRI and TVUS for differentiation of malignant from benign adnexal lesions. Eur Radiol 8:39-44

29 Sohaib SA, Mills TD, Sahdev A et al (2005) The role of magnetic resonance imaging and ultrasound in patients with adnexal masses. Clin Radiol 60:340-348

30 Sohaib SA, Sahdev A, Van Trappen P, Jacobs IJ, Reznek RH (2003) Characterization of adnexal mass lesions on MR imaging. AJR Am J Roentgenol 180:1297-1304

31 Stevens SK, Hricak H, Stern JL (1991) Ovarian lesions: detection and characterization with gadoliniumenhanced MR imaging at 1.5 T. Radiology 181:481-488

32 Thurnher SA (1992) MR imaging of pelvic masses in women: contrast-enhanced vs unenhanced images. AJR Am J Roentgenol 159:1243-1250

33 Trappen V, P. O., Rufford BD, Mills TD et al (2007) Differential diagnosis of adnexal masses: risk of malignancy index, ultrasonography, magnetic resonance imaging, and radioimmunoscintigraphy. Int J Gynecol Cancer 17:61-67

34 Tsili AC, Tsampoulas C, Argyropoulou M et al (2008) Comparative evaluation of multidetector CT and MR imaging in the differentiation of adnexal masses. Eur Radiol 18:1049-1057

35 Uehara T, Takahama J, Marugami N et al (2012) Visualization of ovarian tumors using 3T MR imaging: diagnostic effectiveness and difficulties. Magn Reson Med Sci 11:171-178

36 Yamashita Y, Torashima M, Hatanaka Y et al (1995) Adnexal masses: accuracy of characterization with transvaginal US and precontrast and postcontrast MR imaging. Radiology 194:557-565

37 Zhang H, Zhang GF, He ZY, Li ZY, Zhang GX (2014) Prospective evaluation of 3T MRI findings for primary adnexal lesions and comparison with the final histological diagnosis. Archives of Gynecology and Obstetrics 289:357-364

38 Dodge JE, Covens AL, Lacchetti C et al (2012) Preoperative identification of a suspicious adnexal mass: a systematic review and meta-analysis. Gynecol Oncol 126:157-166 



\title{
Chapter 8
}

\begin{abstract}
Background: Estimating the risk of malignancy is essential in the management of adnexal masses. An accurate differential diagnosis between benign and malignant masses will reduce morbidity and costs due to unnecessary operations, and will improve referral to a gynecologic oncologist for specialized cancer care, which improves outcome and overall survival. The Risk of Malignancy Index is currently the most commonly used method in clinical practice, but has a relatively low diagnostic accuracy (sensitivity $75-80 \%$ and specificity $85-90 \%$ ). Recent reports show that other methods, such as simple ultrasound-based rules, subjective assessment and (Diffusion Weighted) Magnetic Resonance Imaging might be superior to the RMI in the pre-operative differentiation of adnexal masses.
\end{abstract}

Methods/Design: A prospective multicenter cohort study will be performed in the south of The Netherlands. A total of 270 women diagnosed with at least one pelvic mass that is suspected to be of ovarian origin who will undergo surgery, will be enrolled. We will apply the Risk of Malignancy Index with a cut-off value of 200 and a two-step triage test consisting of simple ultrasound-based rules supplemented -if necessary- with either subjective assessment by an expert sonographer or Magnetic Resonance Imaging with diffusion weighted sequences, to characterize the adnexal masses. The histological diagnosis will be the reference standard. Diagnostic performances will be expressed as sensitivity, specificity, positive and negative predictive values and likelihood ratios.

Discussion: We hypothesize that this two-step triage test, including the simple ultrasound-based rules, will have better diagnostic accuracy than the Risk of Malignancy Index and therefore will improve the management of women with adnexal masses. Furthermore, we expect this two-step test to be more cost-effective. If the hypothesis is confirmed, the results of this study could have major effects on current guidelines and implementation of the triage test in daily clinical practice could be a possibility. 


\section{BACKGROUND}

Ovarian cancer is the second most common gynecologic malignancy [1]. In 2008 it was the seventh leading cause of cancer deaths in women worldwide [1,2]. Estimating the risk of malignancy is essential in the management of adnexal masses. Patients with a malignancy should undergo an appropriate staging procedure or debulking surgery carried out in specialized surgical centers. This is associated with a better median survival [3]. Vice versa, patients with a benign lesion may be managed conservatively or with minimal invasive surgery in non-specialized hospitals. This will limit morbidity and avoid unnecessary costs: laparoscopic surgery is associated with less blood loss, shorter hospital stay, and fewer postoperative complications with an improved quality of life and faster return to preoperative functioning [4].

There are several methods to distinguish benign from malignant adnexal masses. The commonly most used method in clinical practice is the Risk of Malignancy Index (RMI) [5-7]. The RMI is an easy to use scoring system that is recommended by many national guidelines in the differential diagnosis of ovarian masses. The RMI combines ultrasound variables, menopausal status and serum CA125 into a score used to predict the risk of ovarian cancer before surgery (Figure 1). The reported sensitivity and specificity of RMI at a cut-off value of 200 are $75-80 \%$ and $85-90 \%$, respectively [8]. This results in an incorrect diagnosis (false positive or false negative) in one out of five women with an adnexal mass. These patients therefore receive inappropriate treatment, potentially leading to increased morbidity and/or mortality.

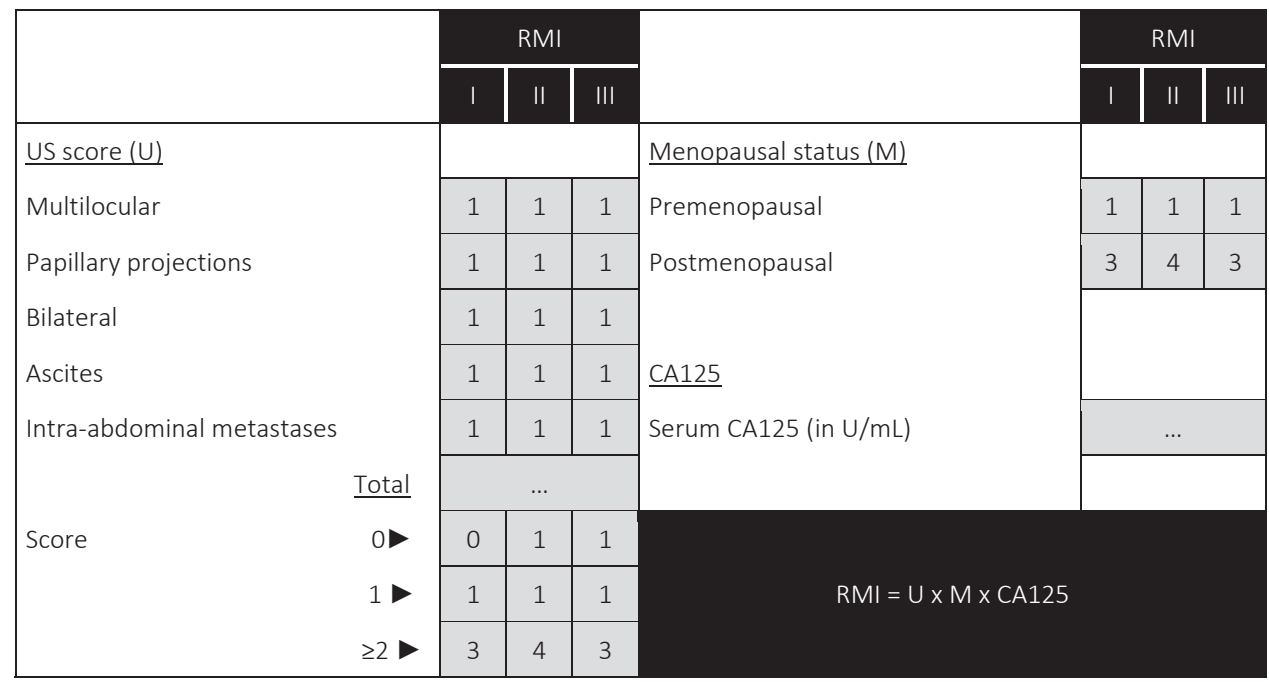

Figure 1. Schematic presentation of three different RMI score algorithms

This figure illustrates 3 different versions of the RMI score system: RMI-I [5], RMI-II [6] and RMI-III [7]. These versions differ from each other in the score attributed to the ultrasound features and menopausal status of the patient. 


\section{Chapter 8}

The 'simple ultrasound-based rules' (simple rules) is another method to differentiate between benignity and malignancy. This method uses different morphological ultrasound features of adnexal masses (without including menopausal status or serum CA125 measurement). It includes 10 rules (Table 1 ); five rules to predict malignancy (Mrules) and five rules to predict a benign tumor (B-rules). If both or none of the $\mathrm{M}$ - and $\mathrm{B}$ rules are met the test is inconclusive $[9,10]$. Simple rules are applicable in approximately $80 \%$ of patients with an ovarian mass and in these cases a sensitivity of $95 \%$ and a specificity of $91 \%$ is achieved in previous studies [11].

Table 1. Benign and malignant ultrasonic features used in simple ultrasound-based rules as proposed by Timmerman et al. [9]

10 Simple ultrasound-based rules

B-features (for predicting a benign tumor)

B1 Unilocular

B2 Presence of solid components, of which largest solid component has largest diameter $<7 \mathrm{~mm}$

B3 Presence of acoustic shadows

B4 Smooth multilocular tumor with largest diameter $<100 \mathrm{~mm}$

B5 No blood flow (color score 1).

M-features (for predicting a malignant tumor)

M1 Irregular solid tumor

M2 Presence of ascites

M3 At least four papillary structures

M4 Irregular multilocular solid tumor with largest diameter $\geq 100 \mathrm{~mm}$

M5 Very strong blood flow (color score 4)

Rule 1: If $\geq 1 \mathrm{M}$-features are present in absence of B-feature(s), the mass is classified as malignant. Rule 2: If $\geq 1 \mathrm{~B}$-features are present in absence of $\mathrm{M}$-feature(s), the mass is classified as benign.

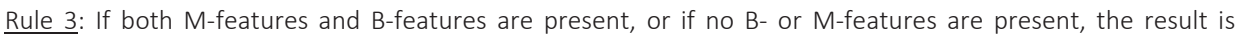
inconclusive and a second stage test is recommended.

In adnexal masses for which the simple rules yield an inconclusive result (unclassifiable masses), subjective assessment by Gray-scale and color Doppler ultrasound imaging by an experienced ultrasound examiner can be used as a second stage test to achieve an optimal diagnostic performance. This subjective assessment is also called 'pattern recognition' [12]. The downside of subjective assessment is that it is experience dependent. Nevertheless, subjective assessment by an expert sonographer seems to be superior to any scoring system or mathematical model when classifying adnexal masses $[10,13,14]$. However, it is neither feasible nor efficient that every patient would have to undergo an expert ultrasonography. Therefore, this method is very well suited as a second stage test. In cases where the simple rules were inconclusive (i.e. masses that are difficult to diagnose) subjective assessment was used successfully with a sensitivity of $91 \%$ and a specificity of $93 \%$ [10]. Several individual reports have confirmed that this two-step triage test is superior to the RMI, especially in terms of sensitivity [15-17]. 
Magnetic Resonance Imaging (MRI) with diffusion weighted sequences is another option for second stage testing of unclassifiable masses. The use of MRI - when interpret by specialized radiologists - also seems to be superior to RMI in the preoperative identification of adnexal masses. A meta-analysis performed by Dodge et al. resulted in an overall sensitivity of $92 \%$ and a specificity of $88 \%$ [18]. The test holds as an advantage that an MRI can not only distinguish a benign from a malignant mass, but can also detect possible metastases in case of a malignancy. Furthermore, MRI can help to select patients who might be more appropriately managed by neoadjuvant chemotherapy [19].

\section{OBJECTIVES}

The primary objective of the SUBSONiC-study (Simple Ultrasound Based ruleS to differentiate OvariaN Cysts) is to test the hypothesis that the simple rules, supplemented -in case of an inconclusive result- with either subjective assessment by an expert sonographer or MRI, will give better diagnostic accuracy than the RMI and therefore will improve the management of women with adnexal masses. Since the RMI is now widely used, any result in favor of the triage test has the potential to alter future clinical practice and reduce costs. Based on the results a cost-effectiveness analysis will be performed.

Secondary objectives are to perform subgroup-analyses for premenopausal and postmenopausal women and to compare the diagnostic accuracy of subjective assessment by an experienced ultrasound examiner with MRI for those cases where the simple ultrasound-based rules were inconclusive. Furthermore, the study aims to assess various forms of interobserver-agreement: in the interpretation of MRI images between radiologists; in the interpretation of simple ultrasound-based rules between the primary ultrasound and the expert ultrasound; and in the subjective assessment between the primary ultrasound and the expert ultrasound. Last objective is to perform translational research and validate new biomarkers in the diagnosis of ovarian cancer.

\section{METHODS/DESIGN}

\section{Study design}

We will perform a prospective multicenter cohort study in the south of The Netherlands; four regional hospitals will participate in the study (Laurentius hospital Roermond, Orbis medical center Sittard, St.Jans hospital Weert and VieCuri Venlo) together with a tertiary referral center (Maastricht University Medical Centre +). In 


\section{Chapter 8}

total, 270 patients will be included within a timeframe of two years. The study will end once the last patient's final diagnosis based on histology is known.

\section{Study population}

Female patients 18 years of age or older are eligible to participate in the study if they are diagnosed in one of the participating centers with at least one pelvic mass that is suspected to be of ovarian origin, and are to undergo surgery in order to obtain a final histological diagnosis. Exclusion criteria are as follows: pregnancy, age under 18 years, a prior bilateral oophorectomy, insufficient or missing data, no informed consent, surgery did not take place or takes place more than 120 days after RMI and simple ultrasoundbased rules are performed, and/or the patient is not able or willing to travel to the center hospital for additional diagnostic procedures.

\section{Study parameters}

The primary research question is the comparison of diagnostic test accuracy between the currently used RMI and a new two-step triage system for the correct differentiation between malignant and benign adnexal masses. Main study parameters are sensitivity, specificity, positive predictive value, negative predictive value and positive and negative likelihood ratios. Sensitivity is defined as the percentage of women with ovarian cancer diagnosed with a malignancy by the RMI and the two-step test, respectively. The positive predictive value is defined as the percentage of patients with a positive test result having malignant disease. The final diagnosis will be based on histology (gold standard).

Based on the results, a cost-effectiveness analysis will be performed.

\section{Study procedures}

Both RMI and simple rules will be performed in the regional hospitals and center hospital by general gynecologists during the same ultrasound scan. For collection of these variables a routine transvaginal ultrasound is sufficient. Transabdominal ultrasonography will be added if the mass is too large to be seen entirely by using only transvaginal ultrasonography. Gray scale and color Doppler ultrasound live images will be used to obtain all morphologic and blood flow variables to characterize each mass by $\mathrm{RMI}$ and by simple rules. The ultrasound will be performed by a general gynecologist or a trainee supervised by a general gynecologist. The sonographer will not be blinded for the serum CA125 level since this is needed to calculate the RMI. Only when the simple rules are inconclusive the patient will be referred to the center hospital for the second stage tests; i.e. subjective assessment and MRI. From previous publications it can be deducted that this will be in approximately $20 \%$ of patients. Subjective assessment will 
be performed by an expert ultrasound examiner. The ultrasound examiner will be a level III sonographer according to the guidelines of the European Federation of Societies for Ultrasound in Medicine and Biology (EFSUMB-criteria), based on -among others- the years of experience, the hours of training and the total number of ultrasound scans performed by the operator [20].

The MRI will be performed at 1.5 Tesla (Intera; Philips Medical Systems, Best, The Netherlands). Conventional 2D T2-weighted sequences in three planes (sagittal, coronal, axial) and 3D T1-weighted sequences in the axial plane will be supplemented with diffusion weighted sequences. The MRI scans will be examined by 2 independent radiologists with experience in $\mathrm{MRI}$, and who are blinded to the outcome of the subjective assessment. Furthermore, a blood sample will be taken for translational research purposes from patients with a mass that cannot be classified by simple rules. These serum samples will be stored for future biomarker studies.

A secured online Case Report Form (CRF) and database ('MACRO') are created in which the demographic data, ultrasound data and MRI data will be stored.

\section{Management of the mass}

The RMI is currently considered the standard diagnostic procedure. Therefore, in case of an RMI outcome of less than 200 - i.e. when a malignancy is not suspected- the mass is currently managed conservatively or with laparoscopy. In case of an RMI outcome of 200 or more the patient is managed together with a gynecological oncologist from the center hospital for comprehensive surgical staging and cytoreductive (debulking) surgery, according to prevailing guidelines. However, it can be foreseen that conflicting results can occur between the RMI and the simple rules combined with the second stage test. If this is the case an individual risk assessment will be made and all conflicting results will be discussed with the patient, after which a gynecological oncologist will be consulted when deemed necessary. Although the RMI currently is considered standard care, we believe that conflicting results cannot be ignored.

\section{Reference standard}

Histopathology is the clinical reference standard used in this study. Therefore, histology of the surgically removed adnexal masses is necessary. The resected masses will be classified according to the World Health Organization guidelines for histology [21]. The pathologist will not have access to the results of the index tests. The specimens obtained for histology will be stored for 30 years, according to prevailing guidelines from the Dutch society for pathology [22]. 


\section{Ethics and dissemination}

The Medical Research Ethics Committee of the Maastricht University Medical Centre in The Netherlands has provided ethical approval for the conduct of the study. Written informed consent will be obtained from all patients before enrollment. The results of the study will be disseminated through international gynecological, radiological and/or oncological peer reviewed publications and conference presentations.

\section{Analysis}

\section{Sample size calculation}

The RMI has a relatively low sensitivity (75-80\%). By reducing the amount of false negative test results the patient will benefit most in terms of prognosis and survival. For the calculation of the sample size we therefore focused on sensitivity as the most important primary objective. This study is designed to have an $80 \%$ power to detect an increase of sensitivity from $79 \%$ for the RMI to $91 \%$ for the two-step triage test with a two-sided $\alpha$ value of 0.05 . Using a matched pair design, it can be estimated that the total discordance between the preoperative diagnosis and the final diagnosis based on histology, obtained by surgery, is $20 \%$ ( $16 \%$ and $4 \%$ respectively). Based on McNemar's test a minimum sample size of 97 women with a malignancy is required [23]. Taking a prevalence of malignancy of $40 \%$ into account, the study will need 243 patients in total. To allow for loss of power of $10 \%$ a total of 270 women will be enrolled in the study.

\section{Statistical analysis}

We will use McNemar's test to determine the statistical significance of differences in sensitivity and specificity between the RMI and the two-step test. For statistical purposes borderline tumors will be classified as malignant tumors. The $95 \%$ confidence intervals for sensitivity and specificity will be obtained using Wilson's interval method [24]. Receiver operating characteristic (ROC) curves will be generated using Medcalc software version 12.7.7.0 (MedCalc Software bvba, Ostend, Belgium) to illustrate the predictive value of the chance of malignancy when using the RMI, simple rules, subjective assessment and MRI. The method described by DeLong et al. will be used for the calculation of the difference between two AUCs [25]. For the subgroup analysis of preand postmenopausal women stratification will be applied. The interobserver-agreement will be evaluated with Cohen's kappa (kappa values of 0.81-1.0 indicate very good agreement, kappa values of $0.61-0.80$ good agreement, kappa values of $0.41-0.60$ moderate agreement, kappa values of 0.00-0.40 poor agreement) [26]. Two-tailed Pvalues of $\leq 0.05$ will be considered statistically significant for all statistical comparisons.

The economic evaluation will explore the potential cost-effectiveness of the triage test compared to RMI. Incremental cost-effectiveness is expressed as difference in the number of correct diagnosis (i.e. either true positive or true negative for malignancy 
based on histology) between both methods. With the usual care (RMI) 83\% of all adnexal masses are diagnosed correctly, versus $96 \%$ with simple rules. The consequences of the difference in correct diagnosed patients between the various test are recharged in the treatment (costs of surgical management and hospital stay following surgery) and will therefore lead to a difference in cost-effectiveness.

\section{DISCUSSION}

Currently the RMI is the most frequently used method to distinguish benign from malignant adnexal masses. However, because of its low sensitivity (75-80\%), malignant tumors will be wrongfully diagnosed as benign in a substantial amount of cases. Subjecting these patients to a laparoscopy can induce spill of cyst fluid, which will deteriorate the prognosis of the patient [27]. The aim of this study is to compare the diagnostic accuracy of different diagnostic methods for differentiating benign from malignant adnexal masses. We will test the hypothesis that a two-step triage test consisting of simple rules, if necessary followed by subjective assessment or MRI, will have better diagnostic accuracy than the RMI and therefore will improve the management of women with adnexal masses. Furthermore, a more correct diagnosis will avoid unnecessary costs due to inappropriate or repeat operations. However, unacceptable high costs due to the use of multiple technical examinations should also be avoided. Therefore, we will also examine the cost-effectiveness of changing the diagnostic procedure.

Indirect, we hope to achieve a decrease in peri-operative morbidity and increase of quality of life by diminishing overtreatment, and also an increase in five-year diseasefree and overall survival due to improved referral to a gynecological oncologist. However, this study is not powered for these analyses. As such, we will not be able to draw any conclusions with regards to these endpoints.

This study would be the first study that investigates the role of simple rules as a triage test in a geographic referral system. When the hypothesis is confirmed, the results of this study could have major effects on current guidelines and implementation of the triage test in daily clinical practice could be possible. Hospitals will be able to use either simple rules in combination with subjective assessment or in combination with MRI, depending on the expertise present and the sources available in each hospital or region. 


\section{REFERENCES}

1. Ferlay J, Shin HR, Bray F, Forman D, Mathers C, DM P. GLOBOCAN 2008 v2.0, Cancer Incidence and Mortality Worldwide: IARC CancerBase No. 10. 2010 [http://globocan.iarc.fr].

2. Siegel R, Naishadham D, Jemal A. Cancer statistics, 2012. CA Cancer J Clin. 2012;62:10-29.

3. Woo YL, Kyrgiou M, Bryant A, Everett T, Dickinson HO. Centralisation of services for gynaecological cancer. Cochrane Database Syst Rev. 2012;3:CD007945.

4. Weber S, McCann CK, Boruta DM, Schorge JO, Growdon WB. Laparoscopic surgical staging of early ovarian cancer. Rev Obstet Gynaecol. 2011;4:117-22.

5. Jacobs I, Oram D, Fairbanks J, Turner J, Frost C, Grudzinskas JG. A risk of malignancy index incorporating CA 125, ultrasound and menopausal status for the accurate preoperative diagnosis of ovarian cancer. $\mathrm{Br}$ J Obstet Gynaecol. 1990;97:922-9.

6. Tingulstad S, Hagen B, Skjeldestad FE, Onsrud M, Kiserud T, Halvorsen T, et al. Evaluation of a risk of malignancy index based on serum CA125, ultrasound findings and menopausal status in the preoperative diagnosis of pelvic masses. Br J Obstet Gynaecol. 1996;103:826-31.

7. Tingulstad S, Hagen B, Skjeldestad FE, Halvorsen T, Nustad K, Onsrud M. The risk-of-malignancy index to evaluate potential ovarian cancers in local hospitals. Obstet Gynecol. 1999;93:448-52.

8. Dutch guideline epithelial ovarian carcinoma [shttp://www.oncoline.nl/index.php?pagina=/richtlijn/item/ pagina.php\&id=28908\& richtlijn_id=631>].

9. Timmerman D, Testa AC, Bourne T, Ameye L, Jurkovic D, Van Holsbeke C, et al. Simple ultrasound-based rules for the diagnosis of ovarian cancer. Ultrasound Obstet Gynecol. 2008;31:681-90.

10. Timmerman D, Ameye L, Fischerova D, Epstein E, Melis GB, Guerriero S, et al. Simple ultrasound rules to distinguish between benign and malignant adnexal masses before surgery: prospective validation by IOTA group. BMJ. 2010;341:c6839.

11. Kaijser J, Sayasneh A, Van Hoorde K, Ghaem-Maghami S, Bourne T, Timmerman D, et al. Presurgical diagnosis of adnexal tumours using mathematical models and scoring systems: a systematic review and meta-analysis. Hum Reprod Update. 2013;20:449-62.

12. Valentin L. Pattern recognition of pelvic masses by gray-scale ultrasound imaging: the contribution of Doppler ultrasound. Ultrasound Obstet Gynecol. 1999;14:338-47.

13. Van Gorp T, Veldman J, Van Calster B, Cadron I, Leunen K, Amant F, et al. Subjective assessment by ultrasound is superior to the risk of malignancy index (RMI) or the risk of ovarian malignancy algorithm (ROMA) in discriminating benign from malignant adnexal masses. Eur J Cancer. 2012;48:1649-56.

14. Valentin L, Jurkovic D, Van Calster B, Testa A, Van Holsbeke C, Bourne T, et al. Adding a single CA 125 measurement to ultrasound imaging performed by an experienced examiner does not improve preoperative discrimination between benign and malignant adnexal masses. Ultrasound Obstet Gynecol. 2009;34:345-54.

15. Testa A, Kaijser J, Wynants L, Fischerova D, Van Holsbeke C, Franchi D, et al. Strategies to diagnose ovarian cancer: new evidence from phase 3 of the multicentre international IOTA study. Br J Cancer. 2014;111:680-8.

16. Sayasneh A, Wynants L, Preisler J, Kaijser J, Johnson S, Stalder C, et al. Multicentre external validation of IOTA prediction models and RMI by operators with varied training. Br J Cancer. 2013;108:2448-54.

17. Ameye L, Timmerman D, Valentin L, Paladini D, Zhang J, Van Holsbeke C, et al. Clinically oriented threestep strategy for assessment of adnexal pathology. Ultrasound Obstet Gynecol. 2012;40:582-91.

18. Dodge JE, Covens AL, Lacchetti C, Elit LM, Le T, Devries-Aboud M, et al. Preoperative identification of a suspicious adnexal mass: a systematic review and meta-analysis. Gynecol Oncol. 2012;126:157-66.

19. Qayyum A, Coakley FV, Westphalen AC, Hricak H, Okuno WT, Powell B. Role of CT and MR imaging in predicting optimal cytoreduction of newly diagnosed primary epithelial ovarian cancer. Gynecol Oncol. 2005;96:301-6.

20. EFSUMB. Minimum training recommendations for the practice of medical ultrasound. Ultraschall Med. 2006;27:79-105. 
21. WHO. World Health Organization classification of tumours. Pathology and genetics of the breast and female genital organs. 2003.

22. Commission Quality \& Occupation of the Dutch Society for Pathology, Advice retention period [http://www.pathology.nl/images/actueel/Publicaties/Kwaliteit/Adviesbewaartermijnendefinitief_juli_20 10.pdf].

23. Rosner, B. Fundamentals of Biostatistics. 7th ed. Boston: Brooks/Cole; 2010.

24. Newcombe RG. Two-sided confidence intervals for the single proportion: comparison of seven methods. Stat Med. 1998;17:857-72.

25. DeLong ER, DeLong DM, Clarke-Pearson DL. Comparing the areas under two or more correlated receiver operating characteristic curves: a nonparametric approach. Biometrics. 1988;44:837-45.

26. Brennan P, Silman A. Statistical methods for assessing observer variability in clinical measures. BMJ. 1992;304:1491-4.

27. Vergote I, De Brabanter J, Fyles A, Bertelsen K, Einhorn N, Sevelda P, et al. Prognostic importance of degree of differentiation and cyst rupture in stage I invasive epithelial ovarian carcinoma. Lancet. 2001;357:176-82. 


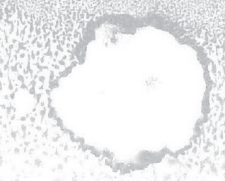

Chapter

Simple rules, not so simple;

The use of International Ovarian Tumor Analysis (IOTA) terminology and simple rules in experienced hands in a prospective multicenter cohort study 


\title{
Chapter 9
}

\begin{abstract}
Objectives: To analyse how well untrained examiners - i.e. with no experience in the use of either International Ovarian Tumor Analysis (IOTA) terminology or simple ultrasoundbased rules (simple rules)- are able to apply IOTA terminology and simple rules. And to assess the level of agreement between untrained examiners and an expert examiner for utilization of the IOTA terminology and subsequent classification of adnexal masses by IOTA simple rules.
\end{abstract}

Methods: This was a prospective multicentre cohort study enrolling women with at least one pelvic mass suspected to be of ovarian origin. An ultrasound scan was performed by one of the non-expert examiners. Ultrasound features were recorded by using the IOTA nomenclature, after which the ultrasonographer classified the mass according to simple rules. Subsequently, a second ultrasound scan was done by an expert examiner following the same procedure. The descriptions of ultrasound features and outcome of simple rules as evaluated by the expert examiner were used as reference standard for our comparisons. Interobserver agreement was evaluated with Fleiss's kappa and the percentage agreement between observers.

Results: A total of 50 consecutive women were included in the study. We observed 46 errors in the description of the ovarian mass when untrained examiners utilized IOTA terminology. In particular, the tumour type was misclassified often $(n=22)$, resulting in a poor interobserver agreement between the non-experts and expert (kappa=0.39, 95\% $\mathrm{Cl}$ 0.244-0.529, percentage of agreement $=52.0 \%$ ). Furthermore, misinterpretation of the simple rules by untrained examiners was observed 57 times. This incorrect use of simple rules ultimately resulted in an erroneous diagnosis in 15 patients (30\%), when compared to the expert's. Therefore, the agreement for classifying the mass as benign, malignant or inconclusive by simple rules was only moderate between the non-experts and the expert (kappa $=0.50,95 \% \mathrm{Cl} 0.300$ to 0.704 , percentage of agreement $=70.0 \%$ ). The level of agreement for all 10 simple rules features varied greatly (kappa index range: $-0.08-0.74$, percentage of agreement range $66-94 \%)$.

Conclusions: Although simple rules are a useful tool to distinguish benign from malignant adnexal masses, they are not that simple for untrained examiners. Training with both IOTA terminology and simple rules is necessary before simple rules can be introduced into guidelines and daily clinical practice. 


\section{INTRODUCTION}

Ultrasound is an indispensable tool in the pre-operative diagnosis of ovarian cancer. Correct characterization of an adnexal mass is important to ensure optimal management of the mass. In order to differentiate benign from malignant ovarian masses many different ultrasound models and scoring systems have been developed over the recent years. However, subjective assessment of ultrasound images by an expert examiner is considered the best way to classify these masses [1]. Nonetheless, it takes years of training and experience to become an expert, and therefore other methods are needed to help less experienced ultrasonographers differentiate benign from malignant adnexal masses.

The International Ovarian Tumor Analysis (IOTA) Group developed ten clinically useful ultrasound rules to characterize ovarian masses as benign or malignant [2; 3]. This method is called simple ultrasound-based rules (simple rules) and contains five ultrasound features suggestive for a benign tumour and five features suggestive for a malignant tumour. Simple rules have been externally validated in several studies in which sensitivities of $73-100 \%$ and specificities of $60-97.5 \%$ have been reported [3-13]. However, in order to apply simple rules, one needs to be familiar with IOTA terms and definitions as described in a consensus paper [14]. Thus far, simple rules have only been validated by examiners trained in the use of IOTA terminology.

The aim of this study was to analyse applicability of simple rules when used by untrained examiners, i.e. with no experience in the use of either IOTA terminology or simple rules. Therefore, four assessments were made (Figure 1):

(1) How well is the IOTA terminology applied by untrained examiners?

(2) How well are the simple rules applied by untrained examiners?

(3) What is the level of agreement between untrained examiners and an expert examiner for utilization of the IOTA terminology?

(4) What is the level of agreement between untrained examiners and an expert examiner for the classification of adnexal masses by IOTA simple rules? 

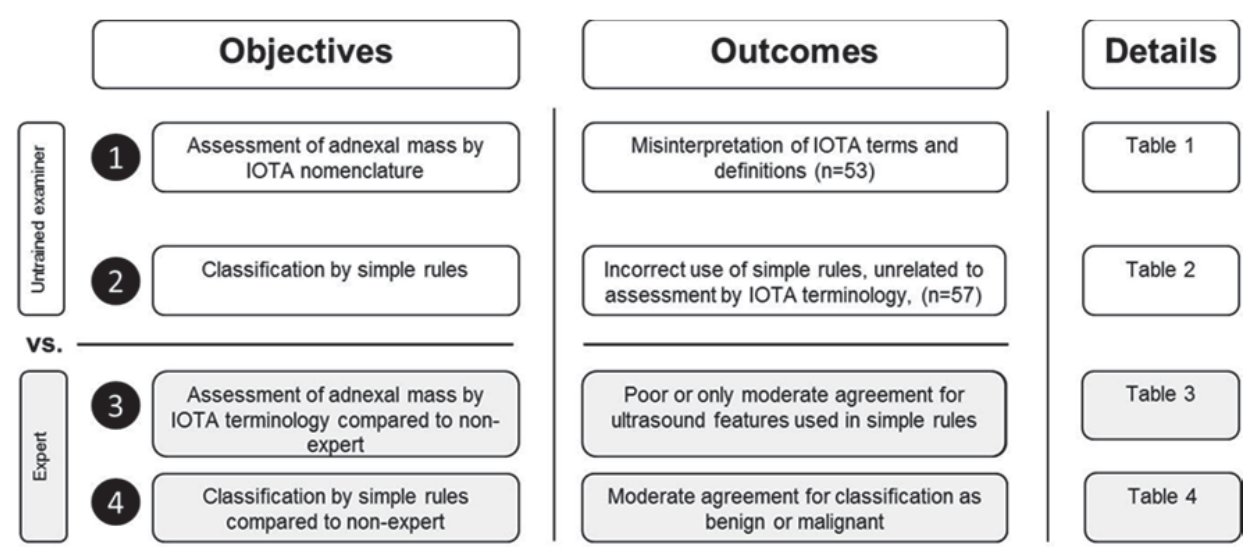

Figure 1. Study objectives and study outcomes

\section{MATERIALS AND METHODS}

This was a prospective multicentre cohort study, called the SUBSONiC-study (Simple Ultrasound Based ruleS to differentiate OvariaN Cysts) [15]. Consecutive patients were recruited in a tertiary referral centre - Maastricht University Medical Centre+ (MUMC+) - and three regional hospitals; Viecuri Venlo, Zuyderland hospital Sittard (formerly: Orbis medical centre Sittard), and St.Jans hospital Weert. Eligible patients had to be 18 years of age or older and diagnosed in one of the participating centres with a pelvic mass suspected to be of ovarian origin. Exclusion took place for: (a) pregnant patients; (b) patients with a prior bilateral oophorectomy in their history; (c) patients from which sufficient data could not be retrieved; (d) patients who did not give or were incapable of giving an informed consent; and (e) patients unable or unwilling to travel to the MUMC+ for a second ultrasound scan by an expert examiner (TVG).

Prior to the start of the study a theoretical training of approximately 2 hours was conducted for the untrained ultrasound examiners participating in the study. During this training IOTA-definitions of the ultrasound features adopted in the simple rules were explained by the expert examiner and some examples were discussed in which definitions and simple rules were practiced.

The study was approved by the local research ethics committees of all participating hospitals (NL44181.068.13). All women included in the study gave written informed consent. STARD guidelines were followed for the conduct, analysis and reporting of our study [16].

Originally we anticipated to perform a prospective multicentre diagnostic test accuracy study for simple rules [15]. We prematurely stopped this study after an interim analysis of 50 patients. The results of the interim analysis are the subject of this article. 


\section{Ultrasound}

All women underwent transvaginal, transrectal and/or transabdominal grey scale and colour Doppler ultrasound. The first ultrasound scan was performed during the initial visit of the patient at the outpatient clinic by a non-expert examiner, i.e. a level I or II examiner according to EFSUMB-criteria (European Federation of Societies for Ultrasound in Medicine and Biology) [17]. A standardized approach was used, in which ultrasound features were recorded meticulously in a pre-defined data collection form using the nomenclature of the IOTA Group [14]. Further details regarding the data collection can be found in Supplementary file S1. After this assessment the ultrasonographer made note which of the simple rules were applicable and what the final diagnosis based on the simple rules was [2; 3]. The simple rules consist of ten ultrasound features; five features suggestive of a benign tumour (B-features) and five suggestive of a malignancy (M-features). The B-features are; unilocular cyst, the presence of solid components where the largest solid component has the largest diameter $<7 \mathrm{~mm}$, the presence of acoustic shadows, smooth multilocular tumour with largest diameter $<100 \mathrm{~mm}$, and no blood flow (colour score 1). The M-features are: irregular solid tumour, presence of ascites, at least four papillary structures, irregular multilocular solid tumour with largest diameter $\geq 100 \mathrm{~mm}$ and very strong blood flow (colour score 4).

If one or more B-features are present in the absence of M-features, the tumour is diagnosed as benign. Vice versa, if one or more $\mathrm{M}$-features are present in the absence of B-features, the mass is classified as malignant. In case both B- and M-features are present, or if none of the ten features is present, the mass is classified as inconclusive.

Furthermore, we collected information regarding age, menopausal status, use of contraceptives (if any), parity, medical history, family history of breast- or ovarian carcinoma, physical complaints and tumour markers.

Subsequently, subjects underwent a second ultrasound, performed by a single level III examiner according to EFSUMB guidelines (TVG) using a Voluson E8 machine (GE Healthcare, Milwaukee, II, USA). This expert followed several courses from the IOTA group and is experienced in use of IOTA nomenclature and simple rules. The same ultrasound features as described in Supplementary file S1 were assessed by this examiner, and a classification of each mass by simple rules was made. The descriptions of ultrasound features and outcome of simple rules as evaluated by the expert examiner were used as reference standard for our comparisons.

All data were entered into a clinical research form and later filed into a specially designed, secured data-collection system (MACRO, Version 4.2.3.3850 InferMed Limited, London, UK). 


\section{Chapter 9}

\section{Statistical analysis}

Interobserver agreement was evaluated with Fleiss's kappa. The kappa value quantifies how much the observed agreement exceeds agreement by chance (values of 0.81-1.0 indicate very good agreement, $0.61-0.80$ good agreement, 0.41-0.60 moderate agreement, 0.00-0.40 poor agreement) [18]. A value of zero indicates agreement equivalent to chance, while negative values indicate observed agreement is less than what is expected by chance. Since kappa values are affected by prevalence, and skewed data can result in low kappa values, we also calculated the absolute percentage agreement between the untrained examiners and expert examiner [19].

In women with bilateral tumours only the tumour with the most complex ultrasound morphology was included in the comparison. If both masses had the same morphology, the mass with the largest size was used for statistical analysis. Borderline tumours were classified as malignant.

All statistical analysis were conducted with IBM SPSS statistics version 20 (IBM Corp, Los Angeles, California, USA) and ReCal3, an online utility that computes interrater reliability coefficients.[20]

\section{RESULTS}

We enrolled 55 patients from September 2014 until September 2015 (Figure 2). Five patients were excluded; one due to missing data (incomplete ultrasound assessment) and four because no ultrasound by the expert examiner took place. Ultimately, 50 patients were included in the study. The median age was 64.5 years (range 27 - 91 years), and 14 (28\%) patients were premenopausal (Supplementary file S2). The first ultrasound was conducted by a resident (level I) in 35 patients, and a gynaecologist (level II) in 15 patients. A total of 17 residents and 9 gynaecologists participated in the study, who included between one and four patients each. The second ultrasound took place between 0 and 108 days (median: 7 days) after the first ultrasound. Simple rules were applicable to 41 patients (82\%) when interpreted by non-experts and in 37 patients (74\%) when interpreted by the expert examiner.

Four assessments were made in accordance with the objectives (Figure 1). First, when evaluating use of IOTA terminology by non-experts, we observed 46 discrepancies within the description of the ovarian mass that were mainly due to misinterpretation of IOTA terms and definitions (Table 1). In particular, tumour type was misclassified often $(n=22)$ and measurements of the mass were not performed in accordance with the IOTA guidelines $(n=20)$. 


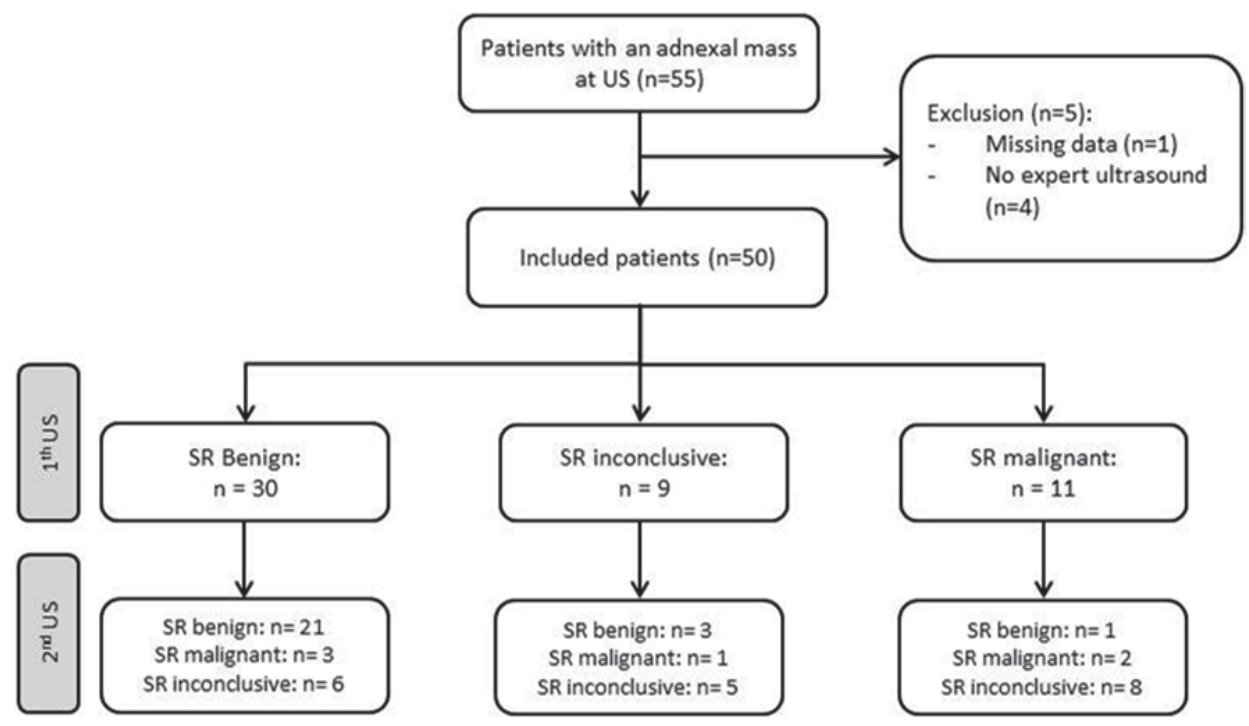

Figure 2. Flow chart of patients included in the study

The first ultrasound was performed by a level I or II examiner (non-expert) with no experience in utilization of IOTA terms and definitions, and was followed by a second ultrasound by an expert (level III examiner).

Abbreviations: US, Ultrasound; SR, IOTA simple ultrasound-based rules.

Table 1. Misinterpretation of terms and definitions as described in the IOTA consensus paper by non-expert examiners for 50 patients

\begin{tabular}{llc}
\hline Type of error & Number of mistakes \\
\hline Wrong tumour type & $22(48 \%)$ \\
Classified as unilocular instead of multilocular & 1 \\
Classified as unilocular instead of unilocular-solid & 5 \\
Classified as unilocular-solid instead of unilocular & 1 \\
Classified as unilocular-solid instead of solid & 1 \\
Classified as multilocular instead of unilocular & 4 \\
Classified as multilocular instead of multilocular-solid & 9 \\
Classified as multilocular-solid instead multilocular & 1 \\
Measurement errors & \\
Ovarian mass not measured in 3 dimensions & 7 \\
Solid component not measured in 3 dimensions & 20 (43\%) \\
No colour Doppler assessment & 3 \\
Measurement of separate loci instead of total mass & 3 \\
Miscellaneous & 2 \\
Incorrect interpretation of 'ascites' & \\
Incorrect interpretation of 'regularity of the inner wall' & 2 \\
\end{tabular}

Second, the classification of adnexal masses by simple rules applied by the untrained examiners was analysed. Regardless of mistakes made in the description of the ovarian mass by IOTA nomenclature, we also observed misinterpretation of simple rules themselves (i.e. wrong translation from description of the mass to the simple rules). 


\section{Chapter 9}

Simple rules were applied incorrectly 57 times by the non-experts (Table 2). This incorrect use of simple rules ultimately resulted in an erroneous diagnosis in 11 patients (22\%); six patients were diagnosed with a benign mass, while correct classification should have resulted in an inconclusive mass; three patients were diagnosed with a malignant mass, while correct classification should have led to an inconclusive mass; and two patients in which simple rules yielded an inconclusive result should have been diagnosed with a benign and malignant mass, respectively.

Table 2. Overview of the incorrect use of the IOTA simple rules by non-experts (independent from the wrongful description of the mass using IOTA nomenclature) and the explanation of mistakes regarding incorrect use of simple rules

\begin{tabular}{|c|c|c|c|}
\hline & $\begin{array}{l}\text { Assigned incorrect } \\
\text { (total number assigned) }\end{array}$ & $\begin{array}{l}\text { Reason for noncompliance with description } \\
\text { (number of times assigned incorrect) }\end{array}$ & \\
\hline B1 & $13(17)$ & $\begin{array}{l}\text { Unilocular solid tumour type } \\
\text { Multilocular type }\end{array}$ & $\begin{array}{l}(11) \\
(2)\end{array}$ \\
\hline B2 & $6(7)$ & $\begin{array}{l}\text { Measurement of } \geq 7 \mathrm{~mm} \\
\text { Unilocular tumour type } \\
\text { Solid tumour type }\end{array}$ & $\begin{array}{l}(4) \\
(1) \\
(1)\end{array}$ \\
\hline B3 & $0(8)$ & - & - \\
\hline B4 & $9(15)$ & $\begin{array}{l}\text { No regular tumour } \\
\text { (Multilocular-)solid tumour type } \\
\text { Unilocular tumour type } \\
\text { Largest diameter }>100 \mathrm{~mm}\end{array}$ & $\begin{array}{l}(5) \\
(2) \\
(1) \\
(1)\end{array}$ \\
\hline B5 & $6(25)$ & $\begin{array}{l}\text { Not applied, while Doppler score was } 1 \\
\text { At colour Doppler score } 2 \\
\text { Only Doppler score for one of the adnexa }\end{array}$ & $\begin{array}{l}(3) \\
(2) \\
(1)\end{array}$ \\
\hline M1 & $12(16)$ & $\begin{array}{l}\text { Multilocular-solid tumour type } \\
\text { Unilocular-solid tumour type } \\
\text { No assessment of inner wall } \\
\text { Multilocular tumour type }\end{array}$ & $\begin{array}{l}(8) \\
(2) \\
(1) \\
(1)\end{array}$ \\
\hline M2 & $1(6)$ & Not applied, while ascites was described & (1) \\
\hline M3 & $2(6)$ & No papillary projections & $(2)$ \\
\hline M4 & $7(12)$ & $\begin{array}{l}\text { Solid tumour type } \\
\text { Multilocular tumour type } \\
\text { Regular tumour } \\
\text { No assessment of inner wall }\end{array}$ & $\begin{array}{l}(3) \\
(2) \\
(1) \\
(1)\end{array}$ \\
\hline M5 & $1(2)$ & Colour Doppler score 3 & (1) \\
\hline
\end{tabular}

Third, when comparing the interpretation of IOTA terminology used by untrained examiners to the evaluation by the expert, we found frequent misclassification of tumour type by untrained examiners. This resulted in poor interobserver agreement regarding tumour type between the non-experts and expert (kappa=0.39, 95\% Cl 0.244-0.529, percentage of agreement $=52.0 \%$ ) (Table 3). Agreement for colour Doppler score was poor as well (kappa=0.19, 95\% $\mathrm{Cl} 0.010-0.380$, percentage of agreement $=46 \%$ ). 
Table 3. Inter-observer variability for observers with different levels of expertise (non-experts vs. an expert) for ultrasound features used to describe an adnexal mass by IOTA terminology

\begin{tabular}{|c|c|c|}
\hline & Agreement & $\begin{array}{l}\text { Kappa value } \\
(95 \% \mathrm{Cl})\end{array}$ \\
\hline $\begin{array}{l}\text { Tumour type } \\
\text { (unilocular/unilocular-solid/multilocular/ multilocular-solid/ solid) }\end{array}$ & $\begin{array}{l}52 \% \\
(26 / 50)\end{array}$ & $\begin{array}{l}0.39 \\
(0.244-0.529)\end{array}$ \\
\hline $\begin{array}{l}\text { Number of loculations } \\
(0 / 1 / 2 / 3 / 4 / 5-10 />10)\end{array}$ & $\begin{array}{l}52 \% \\
(26 / 50)\end{array}$ & $\begin{array}{l}0.37 \\
(0.231-0.501)\end{array}$ \\
\hline $\begin{array}{l}\text { Papillary projections } \\
(0 / 1 / 2 / 3 />3)\end{array}$ & $\begin{array}{l}74 \% \\
(37 / 50)\end{array}$ & $\begin{array}{l}0.13 \\
(-0.059-0.327)\end{array}$ \\
\hline $\begin{array}{l}\text { Acoustic shadow } \\
\text { (yes/no) }\end{array}$ & $\begin{array}{l}74 \% \\
(37 / 50)\end{array}$ & $\begin{array}{l}0.34 \\
(0.063-0.618)\end{array}$ \\
\hline $\begin{array}{l}\text { Inner cyst wall } \\
\text { (regular/irregular/unable to measure) }\end{array}$ & $\begin{array}{l}66 \% \\
(33 / 50)\end{array}$ & $\begin{array}{l}0.41 \\
(0.179-0.633)\end{array}$ \\
\hline $\begin{array}{l}\text { Septations } \\
\text { ( } \leq 3 \mathrm{~mm} / \geq 3 \mathrm{~mm} / \text { no septations })\end{array}$ & $\begin{array}{l}66 \% \\
(33 / 50)\end{array}$ & $\begin{array}{l}0.49 \\
(0.294-0.678)\end{array}$ \\
\hline $\begin{array}{l}\text { Colour Doppler score } \\
\text { (1: no blood flow/2:minimal blood flow/3: moderate blood flow/ } \\
\text { 4: intense blood flow) }\end{array}$ & $\begin{array}{l}46 \% \\
(23 / 50)\end{array}$ & $\begin{array}{l}0.19 \\
(0.010-0.380)\end{array}$ \\
\hline $\begin{array}{l}\text { Ascites } \\
\text { (yes/no) }\end{array}$ & $\begin{array}{l}88 \% \\
(44 / 50)\end{array}$ & $\begin{array}{l}0.55 \\
(0.276-0.831)\end{array}$ \\
\hline $\begin{array}{l}\text { Metastasis } \\
\text { (yes/no) }\end{array}$ & $\begin{array}{l}82 \% \\
(41 / 50)\end{array}$ & $\begin{array}{l}0.08 \\
(-0.197-0.358)\end{array}$ \\
\hline
\end{tabular}

The fourth comparison, in which the interpretation of simple rules applied by nonexperts was compared to the interpretation of the expert, led to a different outcome in 15 patients (30\%). Therefore, the agreement for classifying the mass as benign, malignant or inconclusive by simple rules was only moderate between the non-experts and the expert (kappa $=0.50,95 \% \mathrm{Cl} 0.300$ to 0.704 , percentage of agreement $=70.0 \%$ ). The level of agreement for the B/M-features of the simple rules varied greatly; from an observed agreement less than what is expected by chance to good agreement (Table 4). When the group of non-experts was split up in only level I or only level II examiners the agreement with the expert was good (kappa $=0.63,95 \% \mathrm{Cl} 0.388-0.869$, percentage of agreement $=77.1 \%)$ and poor $(\mathrm{kappa}=0.15,95 \% \mathrm{Cl}-0.232-0.538$, percentage of agreement 53.3\%), respectively. In only 3 cases the exact same simple rules were declared applicable when the interpretation of the non-expert was compared to the expert's. 


\section{Chapter 9}

Table 4. Interobserver-variability for observers with different levels of expertise (non-experts vs. expert) for all ultrasound (B/M-) features included in the IOTA simple rules expressed as Fleiss' kappa index and percentage of agreement

\begin{tabular}{lll}
\hline & Agreement & Kappa value $(95 \% \mathrm{Cl})$ \\
\hline B1 & $76 \%$ & $0.41(0.13-0.68)$ \\
B2 & $86 \%$ & $-0.08(-0.35-0.20)$ \\
B3 & $76 \%$ & $0.34(0.06-0.62)$ \\
B4 & $74 \%$ & $0.22(-0.06-0.49)$ \\
B5 & $66 \%$ & $0.30(0.02-0.57)$ \\
M1 & $70 \%$ & $0.03(-0.25-0.30)$ \\
M2 & $94 \%$ & $0.74(0.46-1.0)$ \\
M3 & $88 \%$ & $0.19(-0.09-0.46)$ \\
M4 & $88 \%$ & $0.63(0.35-0.90)$ \\
M5 & $90 \%$ & $-0.05(-0.33-0.22)$ \\
\hline
\end{tabular}

\section{DISCUSSION}

This is the first study that investigated the interobserver agreement of the simple rules between non-experts, who had no experience with IOTA terminology, and experts in a real-time setting (i.e. not based on video clips). Moreover, we also analyzed the causes of the moderate agreement between non-experts and experts. We encountered two types of mistakes; IOTA definitions were applied wrongfully, and simple rules were not interpreted correctly.

Regarding the first type of mistake, we observed misclassification of tumour type in 22 cases, with a poor interobserver agreement regarding this descriptive item. In a recent study a kappa value of 0.70 was found for tumour type [21]. This concerned agreement between two expert ultrasonographers. We do believe that the interobserver agreement in our population can increase - and mistakes can be prevented- by more comprehensive training. This is also demonstrated by two studies reporting on the impediments ultrasound examiners may encounter when describing adnexal masses by IOTA nomenclature $[22 ; 23]$. Interobserver agreement regarding tumour type in non-experts improved substantially after a consensus meeting. The authors urged for more precise definitions of different descriptive items (e.g. papillary projections, solid components and Doppler colour score). Moreover, the general opinion in the literature is that training should be provided in how to recognize ultrasound features and subsequently describe adnexal masses using IOTA terminology in order to achieve higher interobserver agreement and better diagnostic performance $[11 ; 13 ; 21 ; 24-27]$.

In other studies investigating interobserver agreement for ultrasound features, stored images or video clips instead of real-time ultrasound examinations were used. [24; 28] In these studies the first ultrasound scan is usually made by an expert, 
providing clear images. In the present study the first ultrasound scan was not made by an expert, but by a level I/II examiner, in accordance with normal clinical practice. Interestingly, agreement with the expert was higher for level I examiners when compared to level II examiners, which could be explained by the additional education given to this group as part of their residency. This again stresses the importance of training with IOTA terminology.

The second category of mistakes was a wrong translation from the description of the ovarian mass to the appropriate rules of the simple rules. This is not the first study observing the incorrect application of simple rules. Alcazar et al. found that $12 \%$ of malignant masses were miscategorized as benign by non-expert examiners applying simple rules [6]. Also, Knafel et al. reported that as much as $50 \%$ of their inconclusive results (20/40) were in fact due to misclassification [11].

Nonetheless, most studies conducted up until now have concluded that the simple rules are easy to use in routine clinical practice. The explanation for these contrasting findings in our study is twofold. First, ultrasonographers in other studies were familiar with the nomenclature as described by the IOTA group.[14] This was not the case in our study. It is essential to comprehend all IOTA definitions in order to be able to apply simple rules. Inter-centre differences were observed in a multicentre IOTA study, which -according to the authors - could be due to the difference in examiners' use of IOTA terms.[8] In the Netherlands, the Risk of Malignancy Index is mainly used to differentiate benign and malignant ovarian masses. Since IOTA terminology is not used on a regular basis, this also made it more difficult for the level I/II examiners to interpret the simple rules in the present study.

Second, contrary to our study, many other studies were conducted by ultrasonographers that have some experience with gynaecologic ultrasound. In the Netherlands the emphasis for training in ultrasound is on obstetric ultrasound. Therefore residents and gynaecologists are less experienced in gynaecologic ultrasound. When an ultrasonographer is experienced in gynaecologic ultrasound, some characteristic features of the mass can be anticipated, as is also stated by Tantipalakorn et al [9]. For example, in case of a dermoid cyst an experienced ultrasonographer will easily recognize the echogenic interior as cyst content rather than solid tissue. Thus, the level of experience of the ultrasonographer could bias the interpretation of simple rules, leading to better results in studies where simple rules were validated by examiners more experienced in gynaecologic ultrasound.

Another factor that might have contributed to simple rules being applied incorrectly is the unusual distribution of tumour types in a relative small study population. Three patients suffered from rare benign tumours (struma ovarii, an atypical presentation of a myoma and a benign cyst from an origin unidentified even by pathology) and 5 patients had borderline tumours that are known to be difficult to diagnose.[29] This could also explain why the number of inconclusives was higher than usual (26\% vs. $19 \%$ in other studies) when simple rules were applied by the expert examiner [1]. 


\section{Chapter 9}

Despite the relatively small sample size, our study has the advantage of a prospective design and was conducted in both oncology and non-oncology centres and by nonexperienced and experienced ultrasonographers, which represents day-to-day clinical practice. The number of patients is rather small to perform statistic significant calculations on test performance. Furthermore, histologic conformation of the diagnosis was available in only 37 patients; 23 masses were benign and 14 malignant (including 5 borderline tumours) (Supplementary file S3). However, had we enrolled more patients this would not have given an accurate account of test performance of simple rules, since IOTA terms and definitions and simple rules themselves were not applied correctly by the non-experts.

In conclusion, we believe that ultrasound examiners should be aware of a poorer performance of simple rules if the performing clinician has only little knowledge of the IOTA terms and definitions. In our study a 2 hour training was not enough to fully comprehend the IOTA nomenclature and therefore correctly apply simple rules. Consequently, we believe further training with both IOTA terminology and simple rules is necessary before simple rules can be introduced into daily clinical practice. The introduction of the simple rules in national guidelines should go hand in hand with national training programs or courses. After all, simple rules are just not that simple. 


\section{REFERENCES}

1 Meys EMJ, Kaijser J, Kruitwagen RF et al (2016) Subjective assessment versus ultrasound based models to diagnose ovarian cancer: a systematic review and meta-analysis. Eur J Cancer 58:17-29

2 Timmerman D, Testa AC, Bourne $T$ et al (2008) Simple ultrasound-based rules for the diagnosis of ovarian cancer. Ultrasound Obstet Gynecol 31:681-690

3 Timmerman D, Ameye L, Fischerova D et al (2010) Simple ultrasound rules to distinguish between benign and malignant adnexal masses before surgery: prospective validation by IOTA group. BMJ 341:c6839

4 Fathallah K, Huchon C, Bats AS et al (2011) [External validation of simple ultrasound rules of Timmerman on 122 ovarian tumors]. Gynecol Obstet Fertil 39:477-481

5 Hartman CA, Juliato CR, Sarian LO et al (2012) Ultrasound criteria and CA 125 as predictive variables of ovarian cancer in women with adnexal tumors. Ultrasound Obstet Gynecol 40:360-366

6 Alcazar JL, Pascual MA, Olartecoechea B et al (2013) IOTA simple rules for discriminating between benign and malignant adnexal masses: prospective external validation. Ultrasound Obstet Gynecol 42:467-471

7 Nunes N, Ambler G, Foo X, Naftalin J, Widschwendter M, Jurkovic D (2014) Use of IOTA simple rules for diagnosis of ovarian cancer: meta-analysis. Ultrasound Obstet Gynecol 44:503-514

8 Testa A, Kaijser J, Wynants L et al (2014) Strategies to diagnose ovarian cancer: new evidence from phase 3 of the multicentre international IOTA study. Br J Cancer 111:680-688

9 Tantipalakorn C, Wanapirak C, Khunamornpong S, Sukpan K, Tongsong T (2014) IOTA simple rules in differentiating between benign and malignant ovarian tumors. Asian Pac J Cancer Prev 15:5123-5126

10 Ruiz de Gauna B, Rodriguez D, Olartecoechea B et al (2015) Diagnostic performance of IOTA simple rules for adnexal masses classification: a comparison between two centers with different ovarian cancer prevalence. Eur J Obstet Gynecol Reprod Biol 191:10-14

11 Knafel A, Banas T, Nocun A et al (2015) The Prospective External Validation of International Ovarian Tumor Analysis (IOTA) Simple Rules in the Hands of Level I and II Examiners. Ultraschall Med. 10.1055/s0034-1398773

12 Silvestre L, Martins WP, Candido-Dos-Reis FJ (2015) Limitations of three-dimensional power Doppler angiography in preoperative evaluation of ovarian tumors. J Ovarian Res 8:47

13 Tinnangwattana D, Vichak-Ururote L, Tontivuthikul P, Charoenratana C, Lerthiranwong T, Tongsong T (2015) IOTA Simple Rules in Differentiating between Benign and Malignant Adnexal Masses by Nonexpert Examiners. Asian Pac J Cancer Prev 16:3835-3838

14 Timmerman D, Valentin L, Bourne TH et al (2000) Terms, definitions and measurements to describe the sonographic features of adnexal tumors: a consensus opinion from the International Ovarian Tumor Analysis (IOTA) Group. Ultrasound Obstet Gynecol 16:500-505

15 Meys EM, Rutten IJ, Kruitwagen RF et al (2015) Investigating the performance and cost-effectiveness of the simple ultrasound-based rules compared to the risk of malignancy index in the diagnosis of ovarian cancer (SUBSONiC-study): protocol of a prospective multicenter cohort study in the Netherlands. BMC Cancer 15:482

16 Bossuyt PM, Reitsma JB, Bruns DE et al (2003) Towards complete and accurate reporting of studies of diagnostic accuracy: The STARD Initiative. Ann Intern Med 138:40-44

17 European Federation of Societies for Ultrasound in Medicine, Biology, Education, Committee PS (2006) Minimum training recommendations for the practice of medical ultrasound. Ultraschall Med 27:79-105

18 Brennan P, Silman A (1992) Statistical methods for assessing observer variability in clinical measures. BMJ 304:1491-1494

19 Feinstein AR, Cicchetti DV (1990) High agreement but low kappa: I. The problems of two paradoxes. J Clin Epidemiol 43:543-549

20 Freelon DG (2010) ReCal: Intercoder reliability calculation as a web service. International Journal of Internet Science 5:20-33 


\section{Chapter 9}

21 Sladkevicius P, Valentin L (2015) Interobserver agreement in describing the ultrasound appearance of adnexal masses and in calculating the risk of malignancy using logistic regression models. Clin Cancer Res 21:594-601

22 Zannoni L, Savelli L, Jokubkiene L et al (2014) Intra- and interobserver agreement with regard to describing adnexal masses using International Ovarian Tumor Analysis terminology: reproducibility study involving seven observers. Ultrasound Obstet Gynecol 44:100-108

23 Zannoni L, Savelli L, Jokubkiene L et al (2013) Intra- and interobserver reproducibility of assessment of Doppler ultrasound findings in adnexal masses. Ultrasound Obstet Gynecol 42:93-101

24 Ruiz de Gauna B, Sanchez P, Pineda L, Utrilla-Layna J, Juez L, Alcazar JL (2014) Interobserver agreement in describing adnexal masses using the International Ovarian Tumor Analysis simple rules in a real-time setting and using three-dimensional ultrasound volumes and digital clips. Ultrasound Obstet Gynecol 44:95-99

25 Peces Rama A, Llanos Llanos MC, Sanchez Ferrer ML, Alcazar Zambrano JL, Martinez Mendoza A, Nieto Diaz A (2015) Simple descriptors and simple rules of the International Ovarian Tumor Analysis (IOTA) Group: a prospective study of combined use for the description of adnexal masses. Eur J Obstet Gynecol Reprod Biol 195:7-11

26 Van Holsbeke C, Daemen A, Yazbek J et al (2009) Ultrasound methods to distinguish between malignant and benign adnexal masses in the hands of examiners with different levels of experience. Ultrasound Obstet Gynecol 34:454-461

27 Van Calster B, Van Hoorde K, Froyman W et al (2015) Practical guidance for applying the ADNEX model from the IOTA group to discriminate between different subtypes of adnexal tumors. Facts Views Vis Obgyn 7:32-41

28 Guerriero S, Saba L, Ajossa S et al (2013) Assessing the reproducibility of the IOTA simple ultrasound rules for classifying adnexal masses as benign or malignant using stored 3D volumes. Eur J Obstet Gynecol Reprod Biol 171:157-160

29 Fischerova D, Zikan M, Dundr P, Cibula D (2012) Diagnosis, treatment, and follow-up of borderline ovarian tumors. Oncologist 17:1515-1533 


\section{SUPPLEMENTARY FILE 1}

Data collection form

System: $\quad$ Esaote MyLab25/Phillips EnVisor HD/Voluson E8/Phillips HD15/Phillips HD11

Method: transvaginal/transabdominal/transvaginal and transabdominal/transrectal

Side: $\quad$ unilateral right/unilateral left/median/bilateral

RIGHT

- Most suspect lesion?:

- Origin:

- Size of lesion:

- Painful at ultrasound?:

- Mobility of the cyst:

- Tumour type:

- Number of loculations:

- Largest solid component:

- Papillary projections

- Presence of acoustic shadow:

- Inner wall:

- Septations:

- Incomplete septations:

- Colour Doppler score:

- Ascites:

- Metastasis:

LEFT:

- Most suspect lesion?:

- Origin:

- Size of lesion:

- Painful at ultrasound?:

- Mobility of the cyst:

- Tumour type:

- Number of loculations:

- Largest solid component:

- Papillary projections:

- Presence of acoustic shadow:

- Inner wall:

- Septations:

- Incomplete septations:

- Colour Doppler score:

- Ascites:

- Metastasis: yes/no

ovarian/uterus/salpinx/uncertain/other...

$\ldots \times \ldots \times \ldots m m$.

yes/no

fully mobile/decreased mobility/fully fixated to foundation

unilocular/unilocular-solid/multilocular/multilocular-solid/solid

$0 / 1 / 2 / 3 / 4 / 5-10 />10$

$\ldots \times \ldots \times \ldots \mathrm{mm}$.

$0 / 1 / 2 / 3 />3$

yes/no

regular/irregular/unable to measure

$\leq 3 \mathrm{~mm} / \geq 3 \mathrm{~mm} / \mathrm{no}$ septations

yes/no

1: no blood flow/2:minimal blood flow/3: moderate blood flow/4: intense blood flow)

yes/no

yes/no

yes/no

ovarian/uterus/salpinx/uncertain/other...

... $\mathrm{x} \ldots \mathrm{x} \ldots \mathrm{mm}$.

yes/no

fully mobile/decreased mobility/fully fixated to foundation

unilocular/unilocular-solid/multilocular/multilocular-solid/solid

$0 / 1 / 2 / 3 / 4 / 5-10 />10$

$\ldots \times \ldots \times \ldots \mathrm{mm}$.

$0 / 1 / 2 / 3 />3$

yes/no

regular/irregular/unable to measure

$\leq 3 \mathrm{~mm} / \geq 3 \mathrm{~mm} / \mathrm{no}$ septations

yes/no

1: no blood flow/2:minimal blood flow/3: moderate blood flow/4: intense blood flow)

yes/no

yes/no 


\section{Chapter 9}

SIMPLE RULES [1]

\section{$B$-features}

Yes/No -- Unilocular

Yes/No -- Presence of solid components, of which largest solid component has largest $\varnothing<7 \mathrm{~mm}$

Yes/No -- Presence of acoustic shadow

Yes/No -- Smooth multilocular tumour with largest $\varnothing<100 \mathrm{~mm}$

Yes/No -- Doppler: no blood flow (colour score 1).

M-features

Yes/No -- Irregular solid tumour

Yes/No -- Presence of ascites

Yes/No -- At least four papillary structures

Yes/No -- Irregular multilocular solid tumour with largest $\varnothing \geq 100 \mathrm{~mm}$

Yes/No -- Doppler: very strong blood flow (colour score 4)

\section{REFERENCES:}

1. Timmerman, D., et al., Simple ultrasound-based rules for the diagnosis of ovarian cancer. Ultrasound Obstet Gynecol, 2008. 31(6): p. 681-90. 


\section{SUPPLEMENTARY FILE 2}

Patient characteristics of 50 women with an adnexal mass included in the study

Clinical characteristic

Age (median, range)

$64.5(27-91)$

Menopausal status

Premenopausal

$14(28)$

Postmenopausal

Family history

Breast cancer

$3(6)$

Ovarian cancer

Complaints

$44(88)$

Pain (in abdomen/pelvis/back)

$34(68)$

Regarding intake (difficulty eating/feeling full/nausea/ unexplained weight loss)

Abdominal (bloating/increased abdominal size/palpable mass)

$27(54)$

Bladder (urgency/frequency)

$16(32)$

Intestinal (constipation/diarrhoea)

$19(38)$

Blood loss (irregular menses/postmenopausal blood loss)

$11(22)$

Sexuality (postcoital bleeding/dyspareunia)

CA125 (IU/ml)

Overall (median, range), $n=48$

$36.5(7.0-7148.0)$

Benign (median, range), $n=23$

$37.0(9.1-293.0)$

Malignant (median, range), $n=13$

$115.0(13.0-7148.0)$

Data are given as $n(\%)$ unless noted otherwise 


\section{Chapter 9}

\section{SUPPLEMENTARY FILE 3}

Histopathology of 37 tumours with their corresponding diagnoses based on IOTA simple rules applied by nonexpert examiners (level I and II) and the expert examiner (level III)

\begin{tabular}{|c|c|c|c|c|c|c|c|}
\hline \multirow[t]{2}{*}{ Histopathology } & \multirow[b]{2}{*}{$(n)$} & \multicolumn{3}{|c|}{ SR by non-experts } & \multicolumn{3}{|c|}{ SR by expert } \\
\hline & & Benign & Malignant & Inconclusive & Benign & Malignant & Inconclusive \\
\hline Benign & (23) & & & & & & \\
\hline Serous cystadenoma & (5) & 4 & 1 & - & 4 & - & 1 \\
\hline Endometrioma & (4) & 3 & 1 & - & 4 & - & - \\
\hline Simple cyst & $(2)$ & 1 & - & 1 & 1 & - & 1 \\
\hline Fibroma & $(2)$ & 1 & 1 & - & 1 & - & 1 \\
\hline Mucinous cystadenoma & (3) & 2 & - & 1 & 1 & - & 2 \\
\hline Teratoma & (1) & - & - & 1 & - & - & 1 \\
\hline Inclusion cyst & (1) & 1 & - & - & - & 1 & - \\
\hline Struma ovarii & (1) & 1 & - & - & 1 & - & - \\
\hline Abscess & (1) & - & - & 1 & - & - & 1 \\
\hline Other & (3) & 1 & - & 2 & 1 & 1 & 1 \\
\hline Malignant & (14) & & & & & & \\
\hline Borderline (various) & (5) & 3 & 1 & 1 & 1 & 2 & 2 \\
\hline Serous adenocarcinoma & (4) & - & 3 & 1 & - & 3 & 1 \\
\hline Mucinous adenocarcinoma & $(2)$ & - & 2 & - & - & 2 & - \\
\hline Endometrioid adenocarcinoma & $a(1)$ & - & - & 1 & - & - & 1 \\
\hline Carcinosarcoma & (1) & - & 1 & - & - & 1 & - \\
\hline Adult granulosa cell tumour & (1) & - & 1 & - & - & 1 & - \\
\hline
\end{tabular}

Abbreviations: SR, IOTA simple ultrasound-based rules 

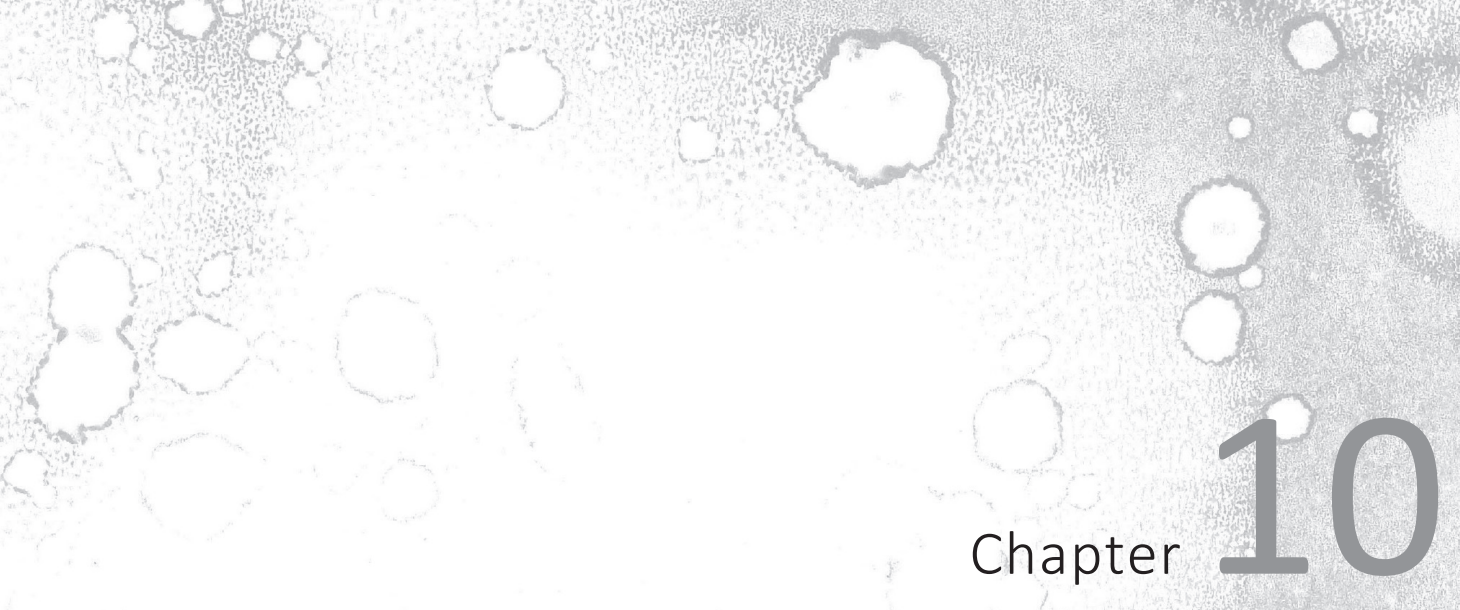

\section{Discussion}
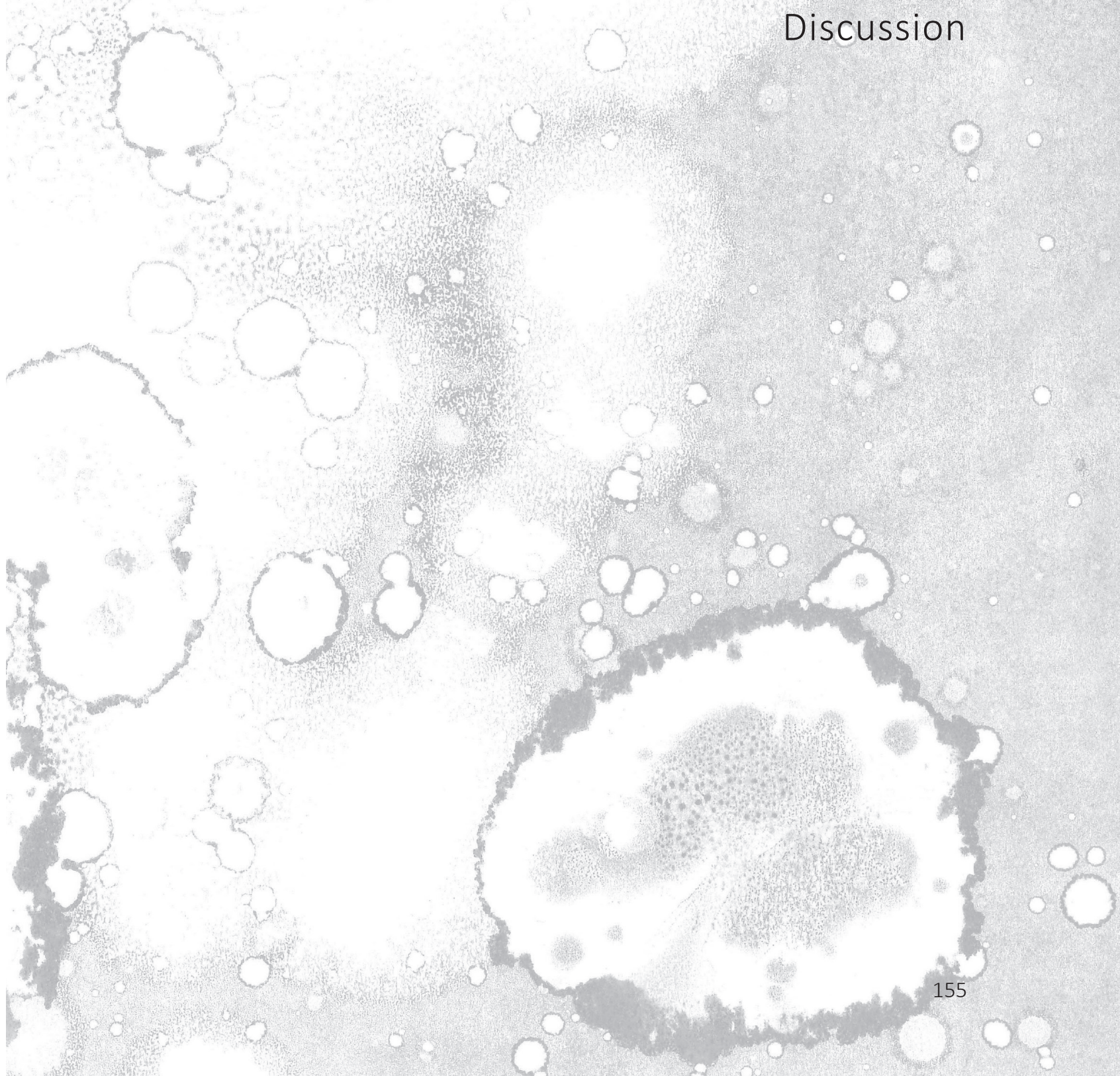
In this thesis we have explored various imaging strategies and modalities in the diagnosis and characterization of ovarian cancer, aiming at optimizing treatment and outcome for the patient.

In part I we discussed computed tomography (CT) based assessment of sarcopenia in relation to surgical outcome and ovarian cancer survival. We found that ovarian cancer patients who lose total muscle mass during neoadjuvant chemotherapy have a worse survival compared to those who maintain or gain muscle mass (Chapter 2). This was the first study to prove this relationship in ovarian cancer. Other studies in lung and pancreatic cancer undergoing palliative chemotherapy reported similar results $[1 ; 2]$. A second finding that was established in both our study and the two reference studies was that a decrease in skeletal muscle index (SMI) over time was predictive of a shorter survival while a single low SMI measurement before start of chemotherapy had no predictive relevance $[1 ; 2]$. In our opinion, measuring muscle loss over time within the individual patient is more reliable for accurate identification of sarcopenic patients than using a general cut-off which doesn't take into account the large variation of muscle mass and variables like obesity and ethnicity within a population [3; 4]. Nevertheless we also focused on investigating the value of a single pretreatment SMI measurement. If sarcopenic patients can be accurately identified, it would open a window of opportunity for an anti-sarcopenia intervention before onset of cancer treatment.

In our second cohort (Chapter 3 ) we chose to use an optimum stratification analysis instead of the median SMI (as in Chapter 2) to define the optimal cut-off point for sarcopenia to predict overall survival (OS). Our results showed that sarcopenia defined as an SMI lower than the optimal cut-off was not predictive of OS and major surgical complications after PDS. Although a strong trend towards a survival disadvantage for sarcopenic patients was seen, other predictors such as residual tumour after PDS and the development of severe complications had a more significant impact on survival outcome. It may be that the impact of baseline sarcopenia measurement on OS will become more apparent when a larger population is studied. The current prevailing opinion in literature is that low skeletal muscle is to a lesser or greater degree associated with a poor prognosis in various tumour types [5-13]. Research has predominantly focused on tumours that have a relationship with nutrition and metabolism such as hepatocellular cancer, pancreaticobiliary cancer and gastrooesophageal cancer. Nevertheless, studies in lung cancer, urothelial cancer, renal cancer and lymphoma have also confirmed a shorter survival in sarcopenic patients suggesting that sarcopenia may play a bigger role than one might expect and clinicians may underestimate its prognostic value $[2 ; 6 ; 7 ; 14-16]$. In gynaecological malignancies studies are scarce and most gynaecologists seem unfamiliar with the role of sarcopenia in endometrial cancer, cervical cancer or ovarian cancer. The multiplicity of cut-off points for sarcopenia that are being used in various cancers makes the interpretation and clinical applicability of the findings quite complicated. SMI cut-off points between 
29 and $42 \mathrm{~cm}^{2} / \mathrm{m}^{2}$ have been reported for adult female patients with solid tumours, resulting in an incidence of sarcopenia of $11-74 \%$ in the studied populations [13]. Prospective and larger studies in a homogeneous population assessing not only body composition on serial CTs, but also nutritional status, physical activity, inflammatory factors (CRP), and chemotherapy response (CA-125) and toxicity may help to better identify patients who are prone to develop sarcopenia. Subsequently randomized controlled trials with nutritional, pharmacological, and/or physical interventions are necessary to establish whether muscle impairment can be prevented or reversed in order to prolong ovarian cancer survival.

Measurements of skeletal muscle change on $\mathrm{CT}$ have proven to be important for prognosis yet time-consuming and hence difficult to implement in daily practice. We evaluated whether measuring the psoas muscle instead of the total skeletal muscle area could be more time efficient (Chapter 4). We found that psoas area is not representative of total muscle area. Although several authors have used the psoas area to assess sarcopenia in other cancers they have not validated the correlation between psoas and total muscle area and we question the reliability of this method. Consequently, the majority has failed to prove a relationship between psoas area assessed sarcopenia and survival [17-24].

In Part II we reviewed current and novel imaging techniques to predict ovarian cancer resectability. Three prospectively developed CT-based models by Ferrandina (models A and B) and by Gerestein for predicting incomplete primary debulking surgery were validated (Chapter 5). Two of the three models performed poor when tested in our validation cohort and none of the three models were sufficiently accurate for clinical decision making. In fact, the radiologists' subjective assessment was as good as any of the models, yet still not accurate enough to rely on for treatment choices. The decision whether a patient will benefit more from primary surgery or primary chemotherapy in the event of an expected incomplete surgery is clinically relevant. Incomplete primary debulking surgeries occur in 8-65\% stressing the need for more accurate assessment tools [25-38]. Ovarian cancer survival relies heavily on the (complete or incomplete) outcome of debulking surgery. Recent studies have shown that diffusion-weighted magnetic resonance imaging (DW-MRI) in gynaecological cancer can detect peritoneal dissemination and metastatic lymph nodes with high accuracy [39-41]. Additionally, clinical applications of combined positron emission tomography magnetic resonance imaging (PET/MRI) are being explored with promising results in exploratory case series [42; 43]. This method combines the strengths of both modalities - molecular imaging technology from positron emission tomography (PET) and anatomic and functional detail from magnetic resonance imaging (MRI) - in one examination. In our small pilot study we explored the diagnostic value of hybrid PET/MRI and DW-MRI for the assessment of ovarian cancer spread in a group of patients undergoing primary or interval debulking surgery (Chapter 6). Both PET/MRI and DW-MRI were feasible 
techniques which showed a tendency towards a superior sensitivity for assessment of peritoneal implants in comparison to CT. However, these favourable results are mainly due to the contribution of MRI; PET is known to be inaccurate in identifying small peritoneal metastases and its negative predictive value is low [44; 45]. It is plausible that the metastases in our study were too small to generate a metabolic signal on PET imaging. DW-MRI on the other hand was far more accurate although our DW-MRI performance was lower than in literature [41]. It could be that at the time of this study, which was one of the first after installation of our PET/MRI device, the DWI protocols with this hybrid technique were suboptimal. It is expected that with optimization of the DW-MRI protocols including motion and breath hold correction techniques its diagnostic performance may improve.

In the final part (Part III) we focused on imaging strategies for the characterization of adnexal masses. A correct discrimination between malignant and benign adnexal masses is pivotal in order to direct further treatment. Traditionally ultrasound has been the gynaecologist's best friend and remains the primary tool to assess adnexal masses worldwide. Nonetheless, different imaging methods have been developed to aid in their evaluation and diagnosis. In a meta-analysis of the existing literature (Chapter 7) we found a high performance for contrast-enhanced MRI in detection of malignant adnexal masses which was in accordance with previous research [46; 47]. The addition of DWI improved its performance although not statistically significant. This may be due to a lack of power conferred by the small scale and few studies that examined DW-MRI. More prospective studies are desired to establish whether DW-MRI can improve the diagnosis and subsequent treatment of women with adnexal tumours.

In our own patient cohort we developed the clinical prospective SUBSONiC study (Chapter 8) to assess the performance of a two-step triage model using simple ultrasound-based rules (Simple Rules) supplemented with expert ultrasound or DW-MRI in case of equivocal findings. The Simple Rules are the product of the International Ovarian Tumour Analysis (IOTA) group who successfully developed these ten ultrasound rules to characterize an adnexal mass. As the study progressed we conducted an interim analysis to see how well the model was applied and we found that the Simple Rules were not as simple as anticipated for an untrained sonographer (Chapter 9). Ultrasound is an indispensable tool for characterization of adnexal masses but is operatordependent. Previous studies investigating simple Rules report high diagnostic performances but have only been validated with expert sonographers [48-57]. We also focused on untrained sonographers and found that the "Simple Rules" were misinterpreted in many cases resulting in a poor interobserver agreement between non-experts and experts for classifying adnexal masses. Due to the high number of misinterpretations and the relatively small study we have not been able to analyze the true diagnostic performance of the two-step triage model. Therefore the question whether expert ultrasound or DW-MRI has additional value to the current standard 
methods remains unanswered. Dedicated training is necessary before Simple Rules can be introduced into guidelines and daily clinical practice. Ensuing the implementation of specialized training programmes for gynaecologists and gynaecological residents, our aim is to restart the SUBSONiC study.

\section{FUTURE PERSPECTIVES}

"A picture is worth a thousand words". Translated to a clinician's point-of-view; cancer treatment and outcome is highly dependent on reliable imaging strategies as is reported in this thesis. Ultrasound and computed tomography are indispensable tools in management of ovarian cancer. Numerous ultrasound-based models have been developed to improve characterization of adnexal masses and more centres - including our own - are achieving high diagnostic performances with expert sonographers. The International Ovarian Tumor Analysis group is continuously developing new models to optimize the diagnosis of adnexal masses. The ADNEX risk model uses clinical and ultrasound variables to estimate the probability that an adnexal mass is benign, borderline, early or advanced ovarian cancer or even metastatic from a different primary tumour. This model is thought to have a superior diagnostic performance than the currently used Risk of Malignancy Index. Both models will be compared in a diagnostic randomised multicentre trial (IOTA-8 or IMPaCT trial) which is going to be performed in the Netherlands in 2017-2019. Additionally specialized training programmes on ultrasound imaging are being established for physicians managing patients with ovarian cancer. This is expected to have a substantial impact on the diagnostic work-up of patients with adnexal masses in the near future.

We have shown that muscle changes as detected on CT have a significant impact on ovarian cancer survival. However the pathophysiology of sarcopenia and the reason why some patients experience more sarcopenia than others is still largely unknown. Current research in our centre aims at understanding the pathophysiologic background of sarcopenia in ovarian cancer patients and is focused on detecting different tumour characteristics in ascites from sarcopenic and non-sarcopenic patients. In this way, intervention studies can be focused on high-risk patients. Recently promising results have been shown with the overnight administration of proteins to elder patients in the prevention of muscle deterioration. Further investigations from our team are planned to culminate into a prospective intervention trial investigating physical and nutritional interventions. Attention to nutritional status, physical activity and performance score before and during treatment is needed in order to ensure the possibility of timely interventions. Moreover technological developments are giving way to the field of big data and radiomics. Radiomics enables the extraction of features from medical images, which can be correlated with tumour phenotypes (radiogenomics). In future this may 
identify even more prognostic factors in the multitude of data from routine CT and MRI sequences to optimize cancer treatment.

The growing demand for reliable patient-tailored prediction of ovarian cancer diagnosis and prognosis is accompanied by the emergence of new imaging techniques. Conversely the radiologist is dealt a greater responsibility to accurately predict resectability. Since surgical outcome is also dependent on the experience and aggressiveness of the surgeon it is pivotal to have a close collaboration between radiologist and gynaecologist in which both are aware of eachothers expertise. PET/MRI seemed promising in prediction of ovarian cancer resectability but is still used in research setting. Furthermore it remains to be seen whether the combined hybrid use of PET/MRI will prove superior to MRI as standalone method also because PET is insensitive to small clinically relevant metastases. DW-MRI is slowly gaining ground in the characterization of adnexal masses and provides an alternative method for ovarian cancer as was shown in our pilot study and reported in peer literature. The experiences are that DW-MRI is accurate and raises the radiologist's confidence for the detection of tumour sites. Larger studies using optimized DW-MRI protocols are indispensable.

\section{CONCLUSIONS AND RECOMMENDATIONS}

An accurate stratification based on imaging can select the individual patient who will benefit from an intervention. We found that patients with ovarian cancer have a worse survival when they lose total muscle mass (sarcopenia) during neoadjuvant chemotherapy compared with those who maintain or gain muscle mass. Although not statistically significant a trend towards an overall survival disadvantage was observed for patients detected as sarcopenic before treatment. Awareness of these findings among gynaecologists and radiologists is imperative to inspire further investigations to confirm our findings. We recommend the consideration of serial CT scans - if feasible with a single slice at the third lumbar vertebra to minimize ionizing radiation - to assess skeletal muscle changes.

Current CT based prediction models are not sufficiently reliable for prediction of ovarian cancer resectability and we do not recommend their use in clinical decisionmaking. New techniques such as PET/MRI and DW-MRI are still in development and research is ongoing to evaluate their role in routine clinical practice. For now DW-MRI can be used as a secondary test in patients in whom resection is deemed questionable on CT and when diagnostic laparoscopy is considered.

Ultrasound remains the upfront modality for the characterization of adnexal masses. Contrast-enhanced MRI and DW-MRI are promising and can help distinguish complex benign from malignant adnexal masses in order to ensure adequate conservative or surgical treatment. Hitherto their use in routine practice is restricted to problem solving. Ultrasound-based Simple Rules have also been developed to improve the 
differentiation between malignant and benign adnexal masses but before they can be introduced into guidelines and daily clinical practice, training programmes are necessary to make gynaecologists familiar with their use. 


\section{REFERENCES}

1 Choi Y, Oh DY, Kim TY et al (2015) Skeletal Muscle Depletion Predicts the Prognosis of Patients with Advanced Pancreatic Cancer Undergoing Palliative Chemotherapy, Independent of Body Mass Index. PLoS One 10:e0139749

2 Stene GB, Helbostad JL, Amundsen T et al (2015) Changes in skeletal muscle mass during palliative chemotherapy in patients with advanced lung cancer. Acta Oncol 54:340-348

3 Jones A, Jr., Shen W, St-Onge MP et al (2004) Body-composition differences between African American and white women: relation to resting energy requirements. Am J Clin Nutr 79:780-786

4 Tan L, Liu SL, Lei SF, Papasian CJ, Deng HW (2012) Molecular genetic studies of gene identification for sarcopenia. Hum Genet 131:1-31

5 Martin L, Birdsell L, Macdonald N et al (2013) Cancer cachexia in the age of obesity: skeletal muscle depletion is a powerful prognostic factor, independent of body mass index. J Clin Oncol 31:1539-1547

6 Psutka SP, Boorjian SA, Moynagh MR et al (2016) Decreased Skeletal Muscle Mass is Associated with an Increased Risk of Mortality after Radical Nephrectomy for Localized Renal Cell Cancer. J Urol 195:270276

7 Psutka SP, Carrasco A, Schmit GD et al (2014) Sarcopenia in patients with bladder cancer undergoing radical cystectomy: impact on cancer-specific and all-cause mortality. Cancer 120:2910-2918

8 van Vledder MG, Levolger S, Ayez N, Verhoef C, Tran TC, ljzermans JN (2012) Body composition and outcome in patients undergoing resection of colorectal liver metastases. Br J Surg 99:550-557

9 Veasey Rodrigues H, Baracos VE, Wheler JJ et al (2013) Body composition and survival in the early clinical trials setting. Eur J Cancer 49:3068-3075

10 Yip C, Dinkel C, Mahajan A, Siddique M, Cook GJ, Goh V (2015) Imaging body composition in cancer patients: visceral obesity, sarcopenia and sarcopenic obesity may impact on clinical outcome. Insights Imaging 6:489-497

11 Iritani S, Imai K, Takai K et al (2015) Skeletal muscle depletion is an independent prognostic factor for hepatocellular carcinoma. J Gastroenterol 50:323-332

12 Itoh S, Shirabe K, Matsumoto $Y$ et al (2014) Effect of body composition on outcomes after hepatic resection for hepatocellular carcinoma. Ann Surg Oncol 21:3063-3068

13 Shachar SS, Williams GR, Muss HB, Nishijima TF (2016) Prognostic value of sarcopenia in adults with solid tumours: A meta-analysis and systematic review. Eur J Cancer 57:58-67

14 Fukushima H, Yokoyama M, Nakanishi Y, Tobisu K, Koga F (2015) Sarcopenia as a prognostic biomarker of advanced urothelial carcinoma. PLoS One 10:e0115895

15 Lanic H, Kraut-Tauzia J, Modzelewski R et al (2014) Sarcopenia is an independent prognostic factor in elderly patients with diffuse large B-cell lymphoma treated with immunochemotherapy. Leuk Lymphoma 55:817-823

16 Murphy RA, Mourtzakis M, Chu QS, Baracos VE, Reiman T, Mazurak VC (2011) Nutritional intervention with fish oil provides a benefit over standard of care for weight and skeletal muscle mass in patients with nonsmall cell lung cancer receiving chemotherapy. Cancer 117:1775-1782

17 Amini N, Spolverato G, Gupta R et al (2015) Impact Total Psoas Volume on Short- and Long-Term Outcomes in Patients Undergoing Curative Resection for Pancreatic Adenocarcinoma: a New Tool to Assess Sarcopenia. J Gastrointest Surg 19:1593-1602

18 Kuroki LM, Mangano M, Allsworth JE et al (2015) Pre-operative Assessment of Muscle Mass to Predict Surgical Complications and Prognosis in Patients With Endometrial Cancer. Ann Surg Oncol 22:972-979

19 Okumura S, Kaido T, Hamaguchi Y et al (2016) Impact of the preoperative quantity and quality of skeletal muscle on outcomes after resection of extrahepatic biliary malignancies. Surgery 159:821-833

20 Peng PD, van Vledder MG, Tsai S et al (2011) Sarcopenia negatively impacts short-term outcomes in patients undergoing hepatic resection for colorectal liver metastasis. HPB (Oxford) 13:439-446 
21 Peyton CC, Heavner MG, Rague JT, Krane LS, Hemal AK (2016) Does Sarcopenia Impact Complications and Overall Survival in Patients Undergoing Radical Nephrectomy for Stage III and IV Kidney Cancer? J Endourol 30:229-236

22 Smith AB, Deal AM, Yu H et al (2014) Sarcopenia as a predictor of complications and survival following radical cystectomy. J Urol 191:1714-1720

23 Valero V, 3rd, Amini N, Spolverato G et al (2015) Sarcopenia adversely impacts postoperative complications following resection or transplantation in patients with primary liver tumors. J Gastrointest Surg 19:272-281

24 Wilson RJ, Alamanda VK, Hartley KG et al (2015) Sarcopenia Does Not Affect Survival or Outcomes in Soft-Tissue Sarcoma. Sarcoma 2015:146481

25 Borley J, Wilhelm-Benartzi C, Yazbek J et al (2015) Radiological predictors of cytoreductive outcomes in patients with advanced ovarian cancer. BJOG 122:843-849

26 Bristow RE, Duska LR, Lambrou NC et al (2000) A model for predicting surgical outcome in patients with advanced ovarian carcinoma using computed tomography. Cancer 89:1532-1540

27 Dowdy SC, Mullany SA, Brandt KR, Huppert BJ, Cliby WA (2004) The utility of computed tomography scans in predicting suboptimal cytoreductive surgery in women with advanced ovarian carcinoma. Cancer 101:346-352

28 Ferrandina G, Sallustio G, Fagotti A et al (2009) Role of CT scan-based and clinical evaluation in the preoperative prediction of optimal cytoreduction in advanced ovarian cancer: a prospective trial. $\mathrm{Br} J$ Cancer 101:1066-1073

29 Gerestein CG, Eijkemans MJ, Bakker J et al (2011) Nomogram for suboptimal cytoreduction at primary surgery for advanced stage ovarian cancer. Anticancer Res 31:4043-4049

30 Janco JM, Glaser G, Kim B et al (2015) Development of a prediction model for residual disease in newly diagnosed advanced ovarian cancer. Gynecol Oncol 138:70-77

31 Jung DC, Kang S, Kim MJ, Park SY, Kim HB (2010) Multidetector CT predictors of incomplete resection in primary cytoreduction of patients with advanced ovarian cancer. Eur Radiol 20:100-107

32 Jung DC, Kang S, Kim SC et al (2013) Use of complex surgical procedures, patterns of tumor spread, and CA-125 predicts a risk of incomplete cytoreduction: a Korean Gynecologic Oncology Group study (KGOG3022). Gynecol Oncol 131:336-340

33 Kim HJ, Choi CH, Lee YY et al (2014) Surgical outcome prediction in patients with advanced ovarian cancer using computed tomography scans and intraoperative findings. Taiwan J Obstet Gynecol 53:343347

34 Mackintosh ML, Rahim R, Rajashanker B et al (2014) CT scan does not predict optimal debulking in stage III-IV epithelial ovarian cancer: a multicentre validation study. J Obstet Gynaecol 34:424-428

35 Mousavi AS, Mazhari MM, Guilani MM, Ghaemmaghami F, Behtash N, Akhavan S (2010) Can primary optimal cytoreduction be predicted in advanced epithelial ovarian cancer preoperatively? World J Surg Oncol 8:11

36 Stashwick C, Post MD, Arruda JS et al (2011) Surgical risk score predicts suboptimal debulking or a major perioperative complication in patients with advanced epithelial ovarian, fallopian tube, or primary peritoneal cancer. Int J Gynecol Cancer 21:1422-1427

37 Suidan RS, Ramirez PT, Sarasohn DM et al (2014) A multicenter prospective trial evaluating the ability of preoperative computed tomography scan and serum CA-125 to predict suboptimal cytoreduction at primary debulking surgery for advanced ovarian, fallopian tube, and peritoneal cancer. Gynecol Oncol 134:455-461

38 Wahner Hendrickson AE, Hawthorne KM, Goode EL et al (2015) Assessment of published models and prognostic variables in epithelial ovarian cancer at Mayo Clinic. Gynecol Oncol 137:77-85

39 Fujii S, Matsusue E, Kanasaki Y et al (2008) Detection of peritoneal dissemination in gynecological malignancy: evaluation by diffusion-weighted MR imaging. Eur Radiol 18:18-23

40 Lin G, Ho KC, Wang JJ et al (2008) Detection of lymph node metastasis in cervical and uterine cancers by diffusion-weighted magnetic resonance imaging at 3T. J Magn Reson Imaging 28:128-135 


\section{Chapter 10}

41 Michielsen K, Vergote I, Op de Beeck K et al (2014) Whole-body MRI with diffusion-weighted sequence for staging of patients with suspected ovarian cancer: a clinical feasibility study in comparison to CT and FDG-PET/CT. Eur Radiol 24:889-901

42 Partovi S, Kohan A, Rubbert C et al (2014) Clinical oncologic applications of PET/MRI: a new horizon. Am J Nucl Med Mol Imaging 4:202-212

43 Queiroz MA, Kubik-Huch RA, Hauser N et al (2015) PET/MRI and PET/CT in advanced gynaecological tumours: initial experience and comparison. Eur Radiol 25:2222-2230

44 De laco P, Musto A, Orazi L et al (2011) FDG-PET/CT in advanced ovarian cancer staging: value and pitfalls in detecting lesions in different abdominal and pelvic quadrants compared with laparoscopy. Eur J Radiol 80:e98-103

45 Kumar Dhingra V, Kand P, Basu S (2012) Impact of FDG-PET and -PET/CT imaging in the clinical decisionmaking of ovarian carcinoma: an evidence-based approach. Womens Health (Lond Engl) 8:191-203

46 Dodge JE, Covens AL, Lacchetti C et al (2012) Preoperative identification of a suspicious adnexal mass: a systematic review and meta-analysis. Gynecol Oncol 126:157-166

47 Medeiros LR, Freitas LB, Rosa DD et al (2011) Accuracy of magnetic resonance imaging in ovarian tumor: a systematic quantitative review. Am J Obstet Gynecol 204:67 e61-10

48 Alcazar JL, Pascual MA, Olartecoechea B et al (2013) IOTA simple rules for discriminating between benign and malignant adnexal masses: prospective external validation. Ultrasound Obstet Gynecol 42:467-471

49 Fathallah K, Huchon C, Bats AS et al (2011) [External validation of simple ultrasound rules of Timmerman on 122 ovarian tumors]. Gynecol Obstet Fertil 39:477-481

50 Hartman CA, Juliato CR, Sarian LO et al (2012) Ultrasound criteria and CA 125 as predictive variables of ovarian cancer in women with adnexal tumors. Ultrasound Obstet Gynecol 40:360-366

51 Knafel A, Banas T, Nocun A et al (2015) The Prospective External Validation of International Ovarian Tumor Analysis (IOTA) Simple Rules in the Hands of Level I and II Examiners. Ultraschall Med. 10.1055/s0034-1398773

52 Nunes N, Ambler G, Foo X, Naftalin J, Widschwendter M, Jurkovic D (2014) Use of IOTA simple rules for diagnosis of ovarian cancer: meta-analysis. Ultrasound Obstet Gynecol 44:503-514

53 Ruiz de Gauna B, Rodriguez D, Olartecoechea B et al (2015) Diagnostic performance of IOTA simple rules for adnexal masses classification: a comparison between two centers with different ovarian cancer prevalence. Eur J Obstet Gynecol Reprod Biol 191:10-14

54 Tantipalakorn C, Wanapirak C, Khunamornpong S, Sukpan K, Tongsong T (2014) IOTA simple rules in differentiating between benign and malignant ovarian tumors. Asian Pac J Cancer Prev 15:5123-5126

55 Testa A, Kaijser J, Wynants L et al (2014) Strategies to diagnose ovarian cancer: new evidence from phase 3 of the multicentre international IOTA study. Br J Cancer 111:680-688

56 Timmerman D, Ameye L, Fischerova D et al (2010) Simple ultrasound rules to distinguish between benign and malignant adnexal masses before surgery: prospective validation by IOTA group. BMJ 341:c6839

57 Tinnangwattana D, Vichak-Ururote L, Tontivuthikul P, Charoenratana C, Lerthiranwong T, Tongsong T (2015) IOTA Simple Rules in Differentiating between Benign and Malignant Adnexal Masses by Nonexpert Examiners. Asian Pac J Cancer Prev 16:3835-3838 

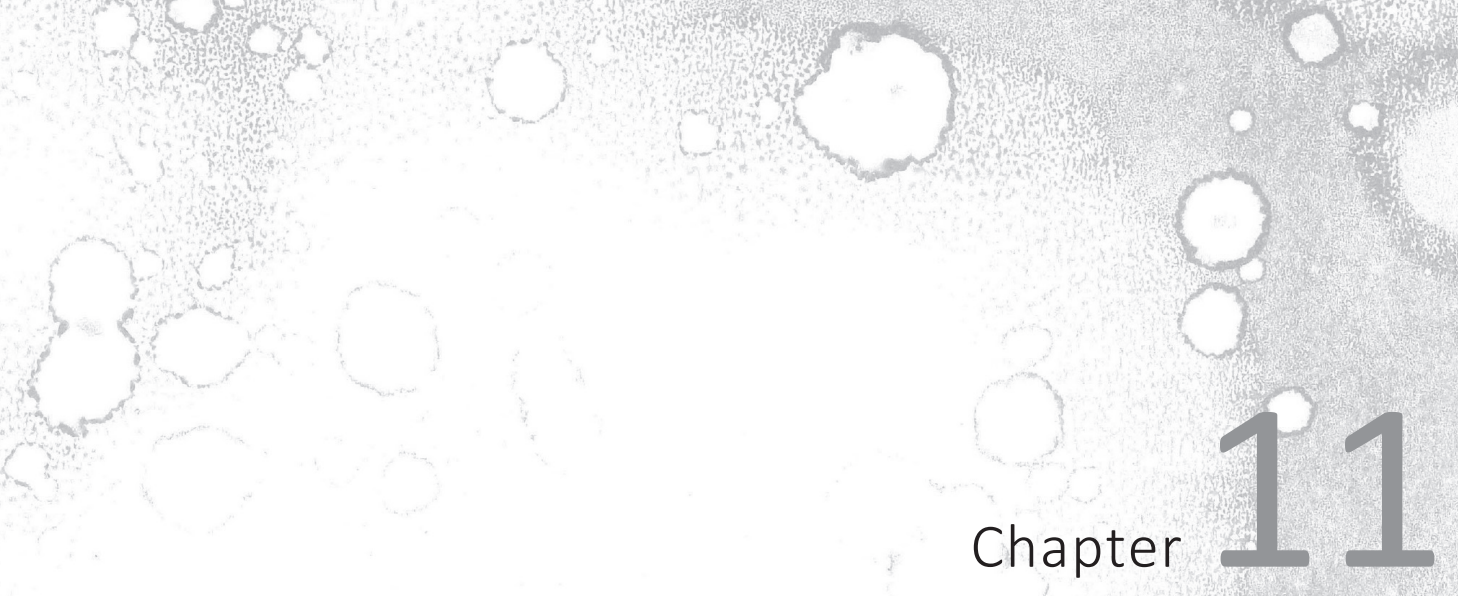

\section{Summary}
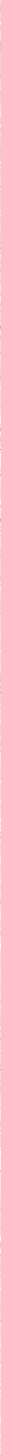
Epithelial ovarian cancer constitutes a great healthcare issue with a high mortality. Therapeutic options depend on the extensiveness of the disease. Surgical removal of all visible tumour sites is the treatment of choice whereas chemotherapy is used as alternative when resection is deemed not viable or impossible. Ultrasound and computed tomography (CT) are the backbone of ovarian cancer diagnosis and characterization. However, other techniques such as magnetic resonance imaging (MRI) and positron emission tomography (PET) are being investigated for diagnostic purposes. In this thesis we evaluate how we can use existing and novel imaging strategies to optimally guide treatment decisions with the objective to improve long term outcome of patients with ovarian cancer. Chapter 1 provides a general introduction and introduces the research questions which form the outline of this thesis.

In part I of this thesis we have used information on skeletal muscle derived from standard CT scans as predictive factors in retrospective ovarian cancer survival analyses. In Chapter 2 we studied advanced ovarian cancer patients ( $n=123$ ) treated with interval debulking surgery and investigated whether changes in skeletal muscle were related to overall survival. We measured surface areas of skeletal muscle on CT scans which were taken before and after treatment with neoadjuvant chemotherapy and compared patients that maintained or gained skeletal muscle with patients that lost skeletal muscle during chemotherapy. Patients with skeletal muscle gain had a significantly better median overall survival of 1431 days compared to patients with skeletal muscle loss who had a median overall survival of 916 days $(p=0.004)$. The predictive influence of skeletal muscle loss was confirmed in multivariable Cox-regression analysis. In this patient group, a skeletal muscle index lower than the median skeletal muscle index at the baseline CT scan before treatment with chemotherapy was not predictive of prognosis. In Chapter 3 we studied our second patient cohort which consisted of 216 patients treated with primary debulking surgery. In this group only a single pretreatment CT scan was available for analysis. Instead of using the median SMI at baseline we performed optimum stratification to find the optimal skeletal muscle index cut-off to define sarcopenia, severe skeletal muscle loss. The aims of the study were to investigate if sarcopenia was associated with overall survival and if sarcopenia was associated with surgical complications. We found that sarcopenia was neither predictive of overall survival nor of surgical complications in ovarian cancer patients undergoing primary debulking surgery. However a strong trend towards a survival disadvantage for patients with sarcopenia was seen but other predictors such as completeness of surgery, treatment in a specialized centre and the development of major surgical complications showed a stronger relationship with overall survival. The effect of sarcopenia on overall survival may be more prominent when studied in a larger cohort of patients. To conclude part I we investigated whether assessment of psoas muscle area reflects total muscle area in Chapter 4. If so, measuring the psoas muscle area alone instead of the total skeletal muscle area on CT could be a quick and easy method to identify sarcopenia. To answer this question we quantified the psoas muscle with two 
methods; firstly with imaging software and secondly manually by measuring the length and width of this muscle. We compared this to quantifications of total muscle area in 150 ovarian cancer patients treated with interval debulking surgery. The correlation between total skeletal muscle area and both psoas area measurement methods was poor. After categorizing patients into muscle loss or gain, kappa agreement was also poor for comparison between total skeletal muscle area and either methods of psoas area. We concluded that a change in psoas muscle area was not representative of a change in total muscle area. Measurement of psoas area should not be used to substitute measurement of total skeletal muscle area to predict sarcopenia and survival in patients with ovarian cancer.

In part II we explored how accurate CT-based prediction models are to predict outcome of primary debulking surgery and we considered two new imaging techniques for the prediction of resectability: diffusion-weighted magnetic resonance imaging (DW$\mathrm{MRI}$ ) and combined positron emission tomography magnetic resonance imaging (PET/MRI). In Chapter 5 three prospectively developed CT prediction models for incomplete surgery (any tumour residual $>1 \mathrm{~cm}$ in diameter) by Ferrandina (models $\mathrm{A}$ and B) and by Gerestein were applied to a validation cohort consisting of 151 patients with advanced epithelial ovarian cancer. All three models showed a limited discriminative ability and the reproducibility of the models was questionable. The models were not sufficiently reliable for clinical decision making. A radiologist's subjective assessment was as accurate as using a prediction model. The high importance of selecting patients who will benefit from primary debulking surgery in combination with the limited performance of CT encouraged us to investigate novel diagnostic modalities in the prediction of ovarian cancer resectability. In Chapter 6 we investigated the diagnostic accuracy of PET/MRI and DW-MRI in comparison with CT for the assessment of surgical resectability. In this prospective pilot study, six patients treated with primary debulking surgery and four patients treated with neoadjuvant chemotherapy and interval debulking surgery were subjected to standard CT scans and a combined PET/MRI scan with DW-MRI. The two novel methods showed a trend towards an improved sensitivity compared to $\mathrm{CT}$ in the detection of peritoneal tumour deposits. However, the results for PET/MRI were mainly a reflection of the contribution of MRI; FDG accumulation was not present in the majority of (small) intraperitoneal tumour sites and PET imaging could not be used reliably to predict negative tumour sites. The predictive value of DW-MRI regarding ovarian cancer resectability in comparison to $\mathrm{CT}$ has shown promise in recent studies and deserves further investigation in larger prospective trials. A higher diagnostic performance is expected with optimization of the DW-MRI scan protocol on dedicated MRI devices.

Part III of the thesis was dedicated to investigating the value of ultrasound and MRI techniques in the characterization of adnexal tumours. Correct differentiation is essential for further treatment decisions. In Chapter 7 we provide the results of our meta-analysis in which we evaluated the diagnostic performance of contrast-enhanced 
MRI and DW-MRI for differentiation between benign and malignant adnexal masses. Thirty-eight datasets were included which resulted in good diagnostic accuracy for contrast-enhanced MRI with a sensitivity of $91 \%$ and a specificity of $89 \%$. A high pooled sensitivity and specificity was also found for DW-MRI with a trend towards an improved diagnostic accuracy compared to MRI. More prospective studies establishing the added value of DW-MRI are still desired. A different strategy towards improving the characterization of adnexal masses incorporating DW-MRI was therefore proposed in Chapter 8 in which we provide the protocol for the prospective multi-centre cohort study SUBSONiC. The International Ovarian Tumour Analysis (IOTA) group successfully developed ten ultrasound rules to characterize an adnexal mass: the Simple Rules. Subjective assessment of an expert sonographer is known to improve the accuracy of adnexal ultrasound further. Our aim was to test the value of a two-step triage test consisting of 1) the Simple Rules, supplemented with 2) subjective assessment by an expert sonographer or DW-MRI in the differentiation between benign and malignant adnexal masses. This triage test was compared with current clinical practice in which the Risk of Malignancy Index is applied. This index incorporates five ultrasound variables, the menopausal status and serum CA-125 to predict the likelihood that an adnexal mass is malignant but results in an incorrect diagnosis in around $20 \%$ of patients. We hypothesized that implementation of the two-step triage test would result in a better diagnostic accuracy than use of the Risk of Malignancy Index. In Chapter 9 we report the results of our interim analysis in which we analyzed one of the secondary objectives of the SUBSONiC study, the interobserver variability between the primary ultrasound executed by an untrained examiner and the subjective assessment of the expert ultrasound executed by an examiner trained in the interpretation of the Simple Rules. The interim analysis provided data for the first 50 consecutive women enrolled in the SUBSONiC study. Frequent misclassification of the tumour type and misinterpretation of the Simple Rules by untrained examiners resulted in a poor interobserver agreement and an incorrect diagnosis (benign, malignant or inconclusive) in 30\% of the patients when compared to the diagnosis made by the expert examiner. Due to the high rates of mistakes found at the interim analysis in both the use of IOTA terminology and application of the Simple Rules, the SUBSONiC study has been halted until further notice. Training programmes for gynaecologists and gynaecological residents are necessary before the SUBSONiC study can be restarted and before assumptions can be made about the diagnostic performance of the model. 



\section{Valorisatie}

Vrouwen die gediagnosticeerd worden met een ovariumcarcinoom hebben slechte overlevingskansen. Dit is met name wanneer de kanker zich presenteert in een gevorderd stadium waarbij de ziekte reeds gemetastaseerd is naar de buik- of pleuraholte. Preoperatieve beeldvorming wordt gebruikt om een inschatting van het karakter en de uitgebreidheid van de ziekte te krijgen. Zo kunnen we de meest geschikte behandeling inzetten. In dit proefschrift wordt onderzocht hoe verschillende beeldvormingstechnieken kunnen bijdragen aan een verbeterde behandeling en daaraan gerelateerd een betere overleving van het ovariumcarcinoom. In deel I tonen wij de nieuwe bevinding dat het verlies van spiermassa (ofwel sarcopenie), gemeten op computer tomografie scans, een belangrijke voorspeller is voor de overleving van patiënten met gevorderd ovariumcarcinoom. Daarnaast beschrijven we in deel II een nieuwe techniek waarbij magnetische resonantie en positron emissie tomografie worden gecombineerd. Deze combinatie leidt tot een betere detectie van tumorlokalisaties in vergelijking met standaard beeldvorming. Uiteindelijk worden er in deel III veelbelovende mogelijkheden gegeven voor beeldvormende strategieën die gebruik maken van echografie modellen en functionele magnetische resonantie technieken om ook in een vroeg stadium de juiste therapie aan te kunnen wijzen voor vrouwen met een massa in het ovarium. In dit valorisatiehoofdstuk proberen we vervolgens de kennis die voortkomt uit deze onderzoeksresultaten te vertalen naar waardevolle toepassingen in onze huidige maatschappij en gezondheidszorg.

Naast de wetenschappelijke relevantie van de resultaten zoals uitvoerig beschreven in dit proefschrift zijn er ook belangrijke sociaal-maatschappelijk bevindingen naar voren gekomen. De laatste jaren is het bestaan en het belang van sarcopenie, spierverlies, steeds meer in de schijnwerpers gekomen met name in samenhang met kanker cachexie en de overleving van patiënten met verschillende soorten kanker. In deel I van dit onderzoek blijkt dat dit ook geldt voor patiënten met ovariumcarcinoom. Patiënten die spiermassa verliezen tijdens de behandeling blijken korter te overleven dan de patiënten waarbij de spiermassa gelijk blijft of toeneemt. Voor vele gynaecologen die patiënten met ovariumcarcinoom behandelen en radiologen die de beeldvorming beoordelen is sarcopenie tot nog toe een onbekende prognostische factor. Dit proefschrift levert dus nieuwe belangrijke informatie voor de arts en voor de patiënt. Daarnaast kunnen de resultaten vertaald worden naar een grotere algemene groep 'gezonde' ouderen waarbij de juiste voeding en voldoende beweging wordt benadrukt voor de beleving van een gezonde oude dag. Onderzoek heeft reeds aangetoond dat sarcopenie ook een rol speelt bij ouderen in het algemeen en bijvoorbeeld bij patiënten die langdurig op de intensive care worden opgenomen. In een maatschappij waar mensen steeds ouder worden en ernaar gestreefd wordt om zo 
lang mogelijk gezond en zelfstandig te blijven is het preventieve behoud van spiermassa een pijler die voor een grote groep interessant is. Het vaststellen van het prognostisch belang van sarcopenie (dit proefschrift) is een eerste stap. Toekomstig onderzoek zal moeten duiden of sarcopenie voorkomen of behandeld kan worden. Specifiek wordt dit onderzoek met name gericht op patiënten met kanker die al een kortere overleving hebben en waar een overlevingswinst directe gevolgen heeft. Echter meer algemeen kan dit onderzoek natuurlijk ook worden voortgezet in de algemene populatie met het oog op behoud van gezondheid en de hiermee onlosmakelijk gepaard gaande preventie van zorgkosten in de toekomst. Een gebruiksklare anti-sarcopenie pil of behandeling zou commercieel interessant zijn maar bestaat voorlopig nog niet. Wel zijn er ontwikkelingen gaande waarbij voeding en voedingssupplementen gecombineerd met beweegprogramma's gericht op behoud en versterking van de spiermassa eventueel ondersteund met medicamenten een steeds belangrijker rol gaan spelen.

Een van de belangrijkste motivaties van veel artsen, inclusief mezelf, om onderzoek te doen is het willen verbeteren van de overleving van kankerpatiënten. Specifiek voor patiënten met een ovariumcarcinoom heb ik in dit proefschrift onderzocht hoe verschillende methoden van beeldvorming hieraan kunnen bijdragen. Wanneer een operatie, met als doel alle lokalisaties van kankerweefsel te verwijderen (zogenaamde debulking), niet toereikend is als gevolg van een te grote tumoruitbreiding, levert dit onnodige morbiditeit, gezondheidskosten en emotionele belasting voor een patiënt op die mogelijk voorkomen had kunnen worden. In die situaties heeft onderzoek al aangetoond dat beter gestart kan worden met chemotherapie. De correcte bepaling van de uitgebreidheid van de kanker vooraf aan een eventuele operatie is daarom essentieel voor het verdere verloop van de behandeling. Per patiënt moet voorafgaand aan de keuze van behandeling worden ingeschat of bij een operatie alle tumorlokalisaties geheel kunnen worden verwijderd. Is dit niet het geval dan dient te worden afgezien van een primaire operatie en wordt patiënte voorbehandeld met chemotherapie.

Natuurlijk is niet alleen de patiënt betrokken bij het ziekteproces maar hebben ook de naaste familie en vrienden, eventuele mantelzorgers en anderen in de directe omgeving een eigen rol hierin. Ook voor hen spelen de emotionele beleving van een ziekteproces, de kwaliteit van leven tijdens en ná de behandeling en eventuele financiële consequenties gerelateerd aan de ziekte en het arbeidsproces mee. De zorg voor de patiënt heeft de hoogste prioriteit maar de bijkomende zorgkosten kunnen niet genegeerd worden in het huidige (en toekomstige) politieke klimaat. Onderzoek naar radiologische technieken die een verbeterde inschatting van tumoruitbreiding kunnen geven dan de standaard beeldvorming en onnodige of onjuiste behandeling kunnen voorkomen hebben derhalve niet alleen een effect op de individuele patiënt maar ook op alle gebruikers van de gezondheidszorg in ons land. Deel II van dit proefschrift laat zien dat de huidige technieken niet altijd een betrouwbare preoperatieve inschatting geven, en dat nieuwe technieken hier mogelijk wel aan bij kunnen dragen. Ook voor wat 


\section{Chapter 12}

betreft patiënten met verdenking op een vroeg-stadium ovariumcarcinoom zijn de huidige technieken onvoldoende accuraat. In deel III van dit proefschrift wordt aandacht besteed aan een nieuwe strategie om de diagnostische accuraatheid te verbeteren zodat de patiënt direct de juiste operatieve behandeling krijgt. Alhoewel de uiteindelijke kosteneffectiviteit van de voorgestelde strategie met het nieuw te implementeren echo-model nog moet worden onderzocht, lijkt deze in eerste instantie voordelig in vergelijking met de bestaande zorgmodellen. 



\section{Dankwoord}

Het heeft misschien wat tijd en moeite gekost, maar dan heb je ook wat. Ik heb de afgelopen jaren een ontzettend leuke tijd gehad tijdens de voorbereiding van dit proefschrift en ik kan iedereen met interesse voor de wetenschap aanraden hetzelfde te doen. Ik wil iedereen die me heeft bijgestaan van harte bedanken en een aantal van deze mensen in het bijzonder.

Dr. T. Van Gorp. Beste Toon, onze gedeelde interesse voor beeldvorming bracht ons aan de praat en van het een kwam het ander. Nu sta je als copromotor naast me bij mijn verdediging. Je hebt me begripvol en kritisch geholpen in het begin en naarmate de tijd vorderde heb je me steeds meer vrijgelaten, precies wat je verwacht van een goede begeleider. Jouw toewijding als oncologisch gynaecoloog heeft me lang doen twijfelen of ik niet dezelfde kant op wilde, maar als echo-expert ben je toch een halve radioloog dus daar kan ik me helemaal in vinden. Ik hoop dat we in de toekomst nog veel mogen samenwerken!

Prof. dr. R.G.H. Beets-Tan. Beste Regina, jij stond aan de wieg van mijn wetenschappelijke carrière. Als jonge student wist je me al enthousiast te maken voor research en daarom was ik erg trots en blij dat jij mijn tweede promotor wilde zijn. Ik zal niet vergeten hoe emotioneel we beiden waren toen ik je vertelde dat ik radioloog wilde worden, een beslissing waar ik geen moment spijt van heb gehad. Je bent een inspirerende vrouw en wetenschapper en ik wil je bedanken voor het vertrouwen dat je me de afgelopen jaren hebt geschonken en de kansen die je me hebt geboden. Je vertrek naar Amsterdam is een groot gemis voor Maastricht en voor mij!

Prof. dr. R.F.P.M. Kruitwagen. Beste Roy, jij gaf me de mogelijkheid om als ANIOS gynaecologie aan de slag te kunnen en daarna als onderzoeker door te stromen in een mooi promotietraject. Op een gegeven moment kwam je met de suggestie dat het misschien interessant zou zijn om eens naar sarcopenie te kijken binnen het ovariumcarcinoom; dit is uitgegroeid van een onschuldig idee naar een groot onderzoek en dat heeft mede bijgedragen aan de succesvolle voltooiing van dit proefschrift. Jouw enthousiasme, nieuwe ideeën en scherpzinnige blik hebben me geholpen om een betere wetenschapper te worden.

De leden van de beoordelingscommissie: Prof. dr. F. Ramaekers, Prof. dr. F. Amant, Prof. dr. C.H.C. Dejong, Prof. dr. A.M.W.J. Schols, Prof. dr. V. Vandecaveye. Bedankt voor jullie tijd en inzet bij de beoordeling van mijn manuscript en jullie aanwezigheid bij mijn verdediging. 
Voormalige collega's van de Gynaecologie in het MUMC. Bedankt dat ik bij jullie aan de slag kon als ANIOS. In het bijzonder wil ik Gerold en Mirjam bedanken voor hun begeleiding op de poli, ik heb veel van jullie geleerd en ook op persoonlijk vlak kon ik bij jullie terecht, ik denk met veel plezier aan deze tijd terug. Brigitte en Sandrijne, bedankt voor het meedenken en includeren van patiënten. Brenda, bedankt voor je hulp bij de SUBSONiC studie. Lennie, als een van de jonge gynaecologen was jij altijd benaderbaar, jij bent zelf inmiddels al gepromoveerd en ik wens je nog veel geluk in je verdere carrière. Ook aan alle AIOS en andere ANIOS waar ik mee heb samengewerkt bedankt voor de tips and tricks van het vak en jullie medewerking aan de studies, ik wens jullie veel succes in de toekomst. Niet te vergeten, Petra, bedankt voor je hulp (en geduld) met het inplannen van afspraken met de heren, je hebt me talloze malen uit de brand geholpen!

Collega's van de Radiologie en Nucleaire Geneeskunde in het MUMC. Ik wil jullie van harte bedanken dat ik zo welkom ontvangen ben als nieuwe AIOS in de groep. Er heerst een geweldige sfeer zowel op de werkvloer als erbuiten. Met name dank aan Michiel en Linda die me de vrijheid hebben gegeven om mijn promotie af te maken (het is gelukt!). Frans, met jou heb ik talloze uren CT's gescoord en jouw enthousiasme voor de radiologie heeft er zeker aan bijgedragen dat ik voor het vak gekozen heb. Stefan, jij hebt me geweldig geholpen om de studie met PET-MRI op te zetten en uit te voeren, dankjewel! Ook wil ik oud-MUMC'ers Monique, Doenja en Max nog bedanken voor hun begeleiding toen ik als student mijn eerste wetenschappelijke stappen heb gezet, jullie ambitie is een inspiratie.

Dank aan alle vrouwen die hebben deelgenomen als proefpersoon!

Mijn paranimfen. Lieve Inge, we kennen elkaar nu al bijna 17 jaar en wonen als echte Eindhovenaren nu alweer 11 jaar in Maastricht. Je bent een betrokken dokter voor je patiënten en de zorgzame moeder van onze vriendengroep. Tijdens onze studie waren we onafscheidelijk en ook nu nog beschouw ik jou als mijn beste vriendin, er kon eigenlijk niemand anders naast me staan als paranimf op deze belangrijke dag. Lieve Evelyne, ik leerde jou kennen als 'het andere meisje van Toon' en we deelden al snel lief en leed binnen het ovarium onderzoek. Je hebt ontzettend veel bereikt, hebt geweldige organisatorische skills en je kunt ook nog eens goed dansen. Jouw promotie is ook bijna afgerond en daarna volgt vast een geweldige carrière als huisarts, heel veel succes!

Alle mede-onderzoekers en WESP-studenten, bedankt voor de gezelligheid en succes in jullie toekomstige carrières. Jorne, wat vind ik het leuk dat jij ook verder gaat met het sarcopenie onderzoek, heel veel succes en hopelijk kunnen we nog vaker samenwerken in de toekomst. Jeanny en Leonie, veel succes met jullie eigen promoties. Steven en David, dankjewel voor jullie hulp en ideeën vanuit chirurgische hoek, zonder de samenwerking met jullie afdeling hadden we nooit zo'n mooie resultaten gevonden. 
Ook aan alle co-auteurs die hebben meegewerkt aan de verschillende studies, van harte bedankt.

Lieve familie en vrienden, bedankt voor de ondersteuning, afleiding en ontspanning, de feestjes, verjaardagen en weekendjes weg, en niet te vergeten de hulp bij de verbouwing van ons huis tijdens mijn promotietijd. Het leven is meer dan werken alleen. Lieve René, ik weet niet hoeveel uren je ons hebt geholpen, je bent een geweldige neef. Hetzelfde geldt voor Igno, ontzettend bedankt voor je hulp. En ook Roxan, Raoul, Juliette, Ingrid, Bart, Paul, Ralph, Manon, Dionne, Sjaak, Ans, Bas, Inge, Erik, Myra, Niels, Piter, Stefan, Renée, Joes dankjewel allemaal. Lieve Sabrina, bedankt voor alle koffie en cocktails. Lieve Valérie, veel succes met jouw eigen promotie, alles komt goed. Lilian en Léon, jullie zijn geweldige schoonouders die altijd voor mij en Lucien klaar staan (maar liever niet meer op het dak!). Léon wat een toeval dat jij en ik op bijna dezelfde dag feest zullen vieren, geniet van je welverdiende pensioen!

Lieve papa en mama, ik weet niet waar ik moet beginnen met jullie te bedanken. Ik heb ontzettend veel respect voor jullie als ouders, als dokters en als mens. Jullie zijn er altijd, voor jullie kinderen, familie en vrienden en niets is te veel gevraagd. Pap, jij bent gewoon de slimste en handigste mens die er bestaat, of het nou gaat om opereren, schrijven of verbouwen. Vele promovendi zijn mij al voorgegaan met jou te bedanken in hun dankwoord maar ik ben vooral erg blij dat jij als mijn vader in het publiek zit bij mijn eigen verdediging. Mam, jij hebt aan een halve blik al genoeg om te weten wat er speelt in mijn hoofd en dat maakt je zo'n goede moeder. Je hebt altijd voor me klaar gestaan en me vooral ook geholpen om tijdens deze drukke periode af en toe even op adem te komen en te genieten. Wabi sabi.

En dan last but definitely not least, Lucien. Mientje. Mijn grote liefde. En nu ook mijn verloofde. Ik hou zo ontzettend veel van jou. Het duurde niet lang voordat ik wist dat ik je nooit meer zou laten gaan. Een lijk in de tuin, een inbraak, een verbouwing of een proefschrift, er is niet veel waar wij nog van schrikken samen. "Sweet sweet baby, life is crazy, but there's one thing I am sure of; that I'm your lady, always baby, and I love you now and ever." 



\section{Curriculum Vitae}

Iris Rutten werd geboren op 10 mei 1988 te Eindhoven. Zij behaalde haar gymnasium diploma in 2006 aan het Lorentz Casimir Lyceum in Eindhoven. Direct daarna startte zij met de opleiding Geneeskunde aan de Universiteit van Maastricht. In het tweede jaar van haar opleiding begon zij met het Honours Programma Wetenschappelijk Onderzoek alwaar ze als student belandde bij Regina Beets-Tan op de afdeling Radiologie van het Academisch ziekenhuis in Maastricht en haar eerste artikels publiceerde tijdens haar wetenschapsstage. Haar klinische semi-arts stage oncologische gynaecologie in het laatste jaar van haar studie besteedde Iris op de afdeling Gynaecologie in Maastricht. In augustus 2012 behaalde zij haar artsexamen en bleef werkzaam bij de Gynaecologie en Obstetrie in Maastricht maar nu in de functie van ANIOS waarbij ze ook ruimte kreeg voor wetenschappelijk onderzoek in samenwerking met Toon van Gorp en Roy Kruitwagen. In April 2014 verruilde ze haar baan als ANIOS om twee jaar lang full-time PhD-onderzoek te doen naar de rol van beeldvorming bij de diagnose en behandeling van het ovariumcarcinoom. De belangrijkste wetenschappelijke resultaten van haar onderzoek worden beschreven in dit proefschrift. In 2015 won ze de "Best Young Researcher Award" op het congres van de European Society of Gynaecological Oncology voor haar onderzoek naar sarcopenie. De combinatie van gynaecologie en radiologie binnen haar onderzoek stelde haar voor een lastig dilemma maar uiteindelijk overwon de voorliefde voor radiologie. In Juni 2016 begon ze aan de opleiding tot Radioloog in het Maastricht Universitair Medisch Centrum te Maastricht. 



\section{List of publications}

Rutten IJG, Bakers FCH, Vöö SA, Mottaghy FM, Beets-Tan RGH, Kruitwagen RFPM, Van Gorp T. Diagnostic value of PET/MRI for assessment of ovarian cancer spread: A feasibility study. (Submitted)

Rutten IJG, Cornelisse S, Viechtbauer W, Kruitwagen RFPM, Beets-Tan RGH, Van Gorp T. Accuracy of Diffusion-weighted and conventional Magnetic Resonance Imaging for differentiation of adnexal masses: a Meta-analysis. (Submitted)

Meys EMJ, Rutten IJG, Kruitwagen RFPM, Slangen BF, Lambrechts S, Mertens HJMM, Nolting WE, Boskamp D, Van Gorp T. Simple rules, not so simple; The use of International Ovarian Tumor Analysis (IOTA) terminology and simple rules in experienced hands in a prospective multicenter cohort study. (Accepted, European Journal of Ultrasound)

Rutten IJG, Ubachs J, Kruitwagen RFPM, Beets-Tan RGH, Olde Damink SWM, Van Gorp T. Psoas muscle area is not representative of total skeletal muscle area in the assessment of sarcopenia in ovarian cancer. Journal of Cachexia, Sarcopenia and Muscle (2017) doi:10.1002/jcsm12180

Rutten IJG, Ubachs J, Kruitwagen RFPM, van Dijk DPJ, Beets-Tan RGH, Massuger, LFAG, Olde Damink SWM, Van Gorp T. The influence of sarcopenia on survival and surgical complications in ovarian cancer patients undergoing primary debulking surgery. European Journal of Surgical Oncology (2016) 43(4):717-724.

Rutten IJG, Kruitwagen RFPM, van Dijk DPJ, Beets-Tan RGH, Olde Damink SWM, Van Gorp T. Loss of skeletal muscle during neoadjuvant chemotherapy is related to decreased survival in ovarian cancer patients. J Cachexia Sarcopenia Muscle (2016), 7:458-466.

Rutten IJG, van de Laar R, Kruitwagen RFPM, Bakers FCH, Ploegmakers MJM, Pappot TWF, Beets-Tan RGH, Massuger LFAG, Zusterzeel PLM, Van Gorp T. Prediction of incomplete primary debulking surgery in patients with advanced ovarian cancer: an external validation study of three models using computed tomography. Gynecol Oncol. 2016; I 40:22-8.

Meys EMJ, Rutten IJG, Kruitwagen RFPM, Slangen BF, Bergmans MGM, Mertens HJMM, Nolting WE, Boskamp D, Beets-Tan RGH, Van Gorp T. Investigating the performance and cost-effectiveness of the Simple Ultrasound-Based Rules compared to the Risk of Malignancy Index in the diagnosis of ovarian cancer (SUBSONiC-study): protocol of a prospective multicenter cohort study in The Netherlands. BMC Cancer. 2015 Jun 26;15(1):482. 
Lambregts DMJ, Heijnen LA, Maas M, Rutten IJG, Martens MH, Backes WH, Riedl RG, Bakers FCH, Cappendijk VC, Beets GL, Beets-Tan RGH. Gadofosveset-enhanced MRI for the assessment of rectal cancer lymph nodes - predictive criteria. Abdom Imaging. 2013 Aug;38(4):720-7.

Maas M, Rutten IJG, Nelemans P, Lambregts DMJ, Cappendijk VC, Beets GL, Beets-Tan $\mathrm{RGH}$. What is the most accurate whole-body imaging modality for assessment of local and distant recurrent disease in colorectal cancer? A meta-analysis: imaging for recurrent colorectal cancer. Eur J Nucl Med Mol Imaging 2011;38(8):1560-1571. 



\section{List of abbreviations}

\begin{tabular}{|c|c|}
\hline Acc & Accuracy \\
\hline AUC & Area Under the Curve \\
\hline BMI & Body Mass Index \\
\hline B-features & Benign Features \\
\hline B-rules & Benign Rules \\
\hline CA-125 & Cancer Antigen 125 \\
\hline ce & Contrast-Enhanced \\
\hline $\mathrm{Cl}$ & Confidence Interval \\
\hline C-Index & Concordance Index \\
\hline CRF & Case Report Form \\
\hline CRP & C-Reactive Protein \\
\hline CT & Computed Tomography \\
\hline DOR & Diagnostic Odds Ratio \\
\hline DWI & Diffusion-Weighted Imaging \\
\hline DW-MRI & Diffusion-Weighted Magnetic Resonance Imaging \\
\hline EFSUMB & European Federation for Ultrasound in Medicine and Biology \\
\hline FATWO & Female Adnexal Tumour of probable Wolffian Origin \\
\hline FDG & Fluorodeoxyglucose \\
\hline FIGO & International Federation of Gynecologic Oncology \\
\hline FN & False Negative \\
\hline FP & False Positive \\
\hline HASTE & Half-fourier Acquisition Single-shot Turbo spin Echo \\
\hline $\mathrm{HR}$ & Hazard Ratio \\
\hline $\mathrm{HU}$ & Hounsfield Unit \\
\hline ICC & Intraclass Correlation Coefficient \\
\hline IDS & Interval Debulking Surgery \\
\hline IMAT & Intramuscular Adipose Tissue \\
\hline IOTA & International Ovarian Tumor Analysis \\
\hline L3 & Third Lumbar Vertebra \\
\hline MA & Muscle Attenuation \\
\hline $\mathrm{MBq}$ & Mega Becquerel \\
\hline M-features & Malignant Features \\
\hline M-rules & Malignant Rules \\
\hline MUMC & Maastricht University Medical Centre \\
\hline NLR & Negative Likelihood Ratio \\
\hline NPV & Negative Predictive Value \\
\hline OR & Odds Ratio \\
\hline
\end{tabular}




\begin{tabular}{|c|c|}
\hline OS & Overall Survival \\
\hline PA & Psoas Area \\
\hline PDS & Primary Debulking Surgery \\
\hline PET & Positron Emission Tomography \\
\hline $\mathrm{PET} / \mathrm{CT}$ & Positron Emission Tomography Computed Tomography \\
\hline $\mathrm{PET} / \mathrm{MRI}$ & Positron Emission Tomography Magnetic Resonance Imaging \\
\hline PLR & Positive Likelihood Ratio \\
\hline PLW & Psoas Length $x$ Width \\
\hline PPV & Positive Predictive Value \\
\hline Q-fat sat & Quick Fat Saturation \\
\hline QUADAS & Quality Assessment of Diagnostic Accuracy Studies \\
\hline $\mathrm{RMI}$ & Risk of Malignancy Index \\
\hline $\mathrm{ROC}$ & Receiver Operating Characteristic \\
\hline SAT & Subcutaneous Adipose Tissue \\
\hline SD & Standard Deviation \\
\hline Sens & Sensitivity \\
\hline SM & Skeletal Muscle \\
\hline SMI & Skeletal Muscle Index \\
\hline SPAIR & SPectral Attenuated Inversion Recovery \\
\hline Spec & Specificity \\
\hline SROC & Summary Receiver Operating Characteristic \\
\hline STARD & Standards for Reporting of Diagnostic Accuracy \\
\hline T1w & T1-Weighted \\
\hline $\mathrm{T} 2 \mathrm{w}$ & T2-Weighted \\
\hline TAT & Total Adipose Tissue \\
\hline TN & True Negative \\
\hline TP & True Positive \\
\hline US & Ultrasound \\
\hline VAT & Visceral Adipose Tissue \\
\hline VIBE & Volumetric Interpolated Breath-hold Examination \\
\hline WHO & World Health Organization \\
\hline WHO-PS & World Health Organization Performance Status \\
\hline
\end{tabular}

